\title{
RARE EARTH NITRIDES \\ AND \\ THEIR APPLICATIONS IN \\ MAGNETIC TUNNEL JUNCTIONS
}

by

\section{FELICIA HELENA ULLSTAD}

\author{
A thesis \\ submitted to the Victoria University of Wellington \\ in fulfilment of the requirements for the degree of \\ Doctor of Philosophy
}

Victoria University of Wellington

2019 

If you wish to make an apple pie from scratch, you must first invent the universe.

Carl Sagan 


\section{Abstract}

In this thesis, we investigate the rare earth nitrides, a family of materials containing many intrinsic ferromagnetic semiconductors, with a particular focus on $\mathrm{GdN}$ and $\mathrm{SmN}$. We investigate the rare earth nitride formation reaction, explore some properties of $\mathrm{GdN}$ and $\mathrm{SmN}$, and finally manufacture and measure magnetic tunnel junctions which incorporate rare earth nitrides. The investigations of the reaction and properties of the materials are used to improve and understand the magnetic tunnel junctions. All samples and devices are grown at room temperature, giving polycrystalline rare earth nitride films.

We show that a rare earth surface can catalytically break the $\mathrm{N}_{2}$ molecule at ambient temperature and low pressures. We follow the nitrogen reacting with the rare earth to form a rare earth nitride in real time via conductance measurements. By comparing the $\mathrm{N}_{2}$ cracking, reaction, and diffusion at both a RE and a REN surface we propose a pressure range in which the nitrogen content in $\mathrm{SmN}$ can be manipulated and conclude that the nitrogen in the top monolayers in a $\mathrm{SmN}$ film is mobile.

In the investigation of $\mathrm{GdN}$ and $\mathrm{SmN}$, we find that the conductivity of $\mathrm{SmN}$ follows the same behaviour as $\mathrm{GdN}$ when changing the $\mathrm{N}_{2}$ pressure during deposition. We follow the conductance change in SmN during deposition and propose a minimum thickness for room temperature deposited SmN films for consistent conductivity measurements. We report structural and magnetic changes in $\mathrm{GdN}$ which has been exposed to $\mathrm{N}$-ions. We also present data on materials making ohmic contact to both 
GdN and SmN.

Finally, we report the manufacturing and investigation of magnetic tunnel junctions using GdN and SmN electrodes with a GaN tunnel barrier. A new pattern design produces 20 devices, in a single deposition, which show consistent behaviour and expands on previous work on this topic. The main focus of the investigation is the J-V characteristics of the magnetic tunnel junctions which shows clear non-linear behaviour arising from tunnelling through the GaN. A Simmons fit to the J-V characteristics yields a barrier height of $0.8 \mathrm{eV}$ and barrier thicknesses close to experimentally determined thicknesses. The J-V characteristics are investigated with changing temperature and changing applied magnetic field to investigate the effect of the ferromagnetism of the GdN and $\mathrm{SmN}$ electrodes. The tunnel magnetoresistance (TMR) of the devices show two contributions, a low-temperature TMR contribution and a $50 \mathrm{~K}$ TMR contribution, and the maximum TMR for all devices are between $100 \%$ to $600 \%$. The devices can withstand current densities up to $4000 \mathrm{~A} / \mathrm{cm}^{2}$ and voltages up to $5 \mathrm{~V}$ which is promising for a wide range of future applications. 


\section{Acknowledgments}

Thank you to all other people who have helped directly or indirectly with the completion of this PhD.

First of all, thank you to my supervisors Joe Trodahl and Ben Ruck and my unofficial additional supervisor Franck Natali for thoughtful discussions and encouragement throughout my PhD.

My colleagues in the Spintronics group and in the Cleanroom group have also contributed to making my PhD experience better by discussing ideas, offering encouragement, and collaborating in the labs. Thank you to Harry Warring, William Holmes-Hewett, Eva Anton, James McNulty, Jay Chan, Jackson Miller, Ali Shaib, Gabriel Bioletti, Chang-Min Lee, Fergus Bramley, Tane Butler, Bart Ludbrook, Simon Granville, Kira Pitman, Natalie Plank, Leo Browning, Campbell Matthews, Conor Burke-Govey, Selvan Murugathas Thanihaichelvan, Jenna Nguyen, Mohsen Maddah, and others who have joined however briefly.

Thank you to everyone in and around the office including Brendan Darby, Walter Somerville, Peter Hauer, Baptiste Auguié, Chhayly Tang, Matt Majic, Johan Grand, Eric Le Ru, Adam Francis, and Xiaohan Chen. Special thanks to Matthias Meyer for helping me with a variety of Python coding problems.

Thank you also to Simon Granville, Shen Chong, and Guy Dubuis for teaching and assisting me in using the PPMS and MPMS over the years, and in particular for giving me free reins in the lab during the last weeks of experiments which proved to be incredibly valuable. 
The technical personnel in the department have been instrumental in the success of both individual specialised projects but also the day-to-day running of the labs. Thank you to the mechanical workshop: Alan Rennie, Nick Grinter, and Alex Puglisi for literally keeping things spinning. Thank you to David Flynn for helping with the SEM and XRD and to Peter Coard for lending me endless cables and connectors and helping me repair electrical equipment.

Special thanks to Leo Browning, Matthew Cryer, and Hamish Colenso for helping me keep my sanity and teaching me to drink coffee. Thank you to Kira Pitman for emotional support and cake.

Before even getting to a PhD I have had the fortune of having fantastic teachers. Thank you in particular to Roland Johansson who sparked my love for physics.

My family and friends have been enormously supportive of my pursuit of science. Thank you in particular to Christina Cedergren, Sven Ullstad, Cristel Ullstad, Joakim Larsson, Emelie Ullstad, Noel Cedergren, and Angelica Jonsson Johansson.

Finally, my biggest supporter during this PhD has been my husband, Patrick Herd. His love kept me going when nothing else did. 


\section{Contents}

1 Introduction 1

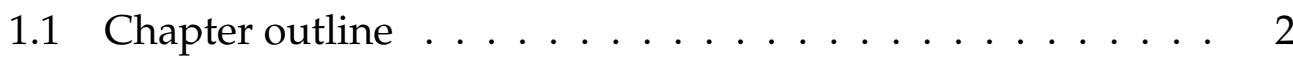

1.2 Publications ..................... 5

2 The rare earth nitrides $\quad 7$

2.1 Structural properties . . . . . . . . . . . . 7

2.2 Magnetic properties . . . . . . . . . . . . . . . 12

2.2.1 General magnetism . . . . . . . . . . . . . . . . . . . 12

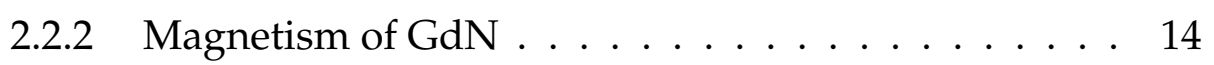

2.2.3 Magnetism of $\mathrm{SmN} \ldots \ldots \ldots$

2.3 Electrical properties . . . . . . . . . . . . . . . . . . 19

3 Experimental methods 25

3.1 Vacuum systems . . . . . . . . . . . . . . . . . . . . . . . . . . .

3.2 UHV systems at VUW . . . . . . . . . . . . . . . . . . . . . . . . . . . .

3.2.1 Chamber maintenance . . . . . . . . . . 28

3.3 Film growth . . . . . . . . . . . . . . . . . . . 29

3.4 Capping layers . . . . . . . . . . . . . . 30

3.5 Patterned samples . . . . . . . . . . . . . . . 31

3.5.1 MTJ device pattern . . . . . . . . . . . . 31

3.5 .2 Photolithography . . . . . . . . . . . . . 34

3.5 .3 Shadow lithography ... . . . . . . . . . 36

3.6 Characterisation and measurements . . . . . . . . . . . 40 
3.6.1 In-situ electrical measurements . . . . . . . . . . . 40

3.6.2 Ex-situ electrical \& magnetic measurements . . . . . . 42

3.6.3 Structural characterisation . . . . . . . . . . . . 46

4 Rare earth reactions with $\mathrm{N}_{2} \quad 47$

4.1 Background of nitriding . . . . . . . . . . . . . 48

4.2 Rare earth changes during nitriding . . . . . . . . . . . . 49

4.2.1 Experimental details . . . . . . . . . . . 50

4.2 .2 Film growth . . . . . . . . . . . . . 51

4.2.3 Structure changes from nitriding Gd . . . . . . . . 54

4.2.4 Conductance changes during nitriding of $\mathrm{Sm}$. . . . . 55

4.3 Extracting nitrogen from $\mathrm{SmN} \ldots \ldots$. . . . . . . . 64

4.3.1 SmN preparation ............... 65

4.3.2 Conductance changes in $\mathrm{SmN} \ldots . . . . . . . .65$

4.4 Discussion . . . . . . . . . . . . . . . . . 70

4.5 Conclusions and future work . . . . . . . . . . 71

5 Rare earth nitride growth $\quad 75$

5.1 Doping SmN during growth . . . . . . . . . . . . 76

5.1 .1 Growth . . . . . . . . . . . . . 76

5.1.2 Pressure dependent conductivity of $\mathrm{SmN} \ldots$. . . . 77

5.2 Conductance evolution of $\mathrm{SmN} \ldots \ldots$. . . . . . . 79

5.3 Sample changes under ion exposure . . . . . . . . . . . . 81

5.3 .1 Growth . . . . . . . . . . . . . . 81

5.3.2 Structural changes from ion exposure . . . . . . . 82

5.3.3 Magnetic changes from ion exposure . . . . . . . 87

5.3.4 Electrical changes from ion exposure . . . . . . . . . 90

5.4 Ohmic contacts to REN films . . . . . . . . . . . . . . . . 90

5.5 Discussion . . . . . . . . . . . . . . . . 93

5.6 Conclusions and future work . . . . . . . . . . . . 95 
CONTENTS ix

6 Magnetic tunnel junctions $\quad 97$

6.1 Background . . . . . . . . . . . . . . . . . . . . . 98

$6.2 \mathrm{GdN} / \mathrm{GaN} / \mathrm{SmN}$ MTJs . . . . . . . . . . . . . . . . . . . . . 104

6.2.1 Structural characterisation . . . . . . . . . . . 106

6.2.2 Magnetic moment . . . . . . . . . . . . . 112

6.2.3 Tunnelling measurements at $0 \mathrm{~T} \ldots$. . . . . . 115

6.2.4 Simmons model of J-V characteristics . . . . . . . . . 130

6.2.5 Magnetoresistance . . . . . . . . . . . . . 137

6.2.6 Device breakdown . . . . . . . . . . . . . 150

6.3 Discussion . . . . . . . . . . . . . . . . . . . . 154

6.4 Conclusions and future work . . . . . . . . . . . . . . 159

7 Summary and Outlook 163

7.1 Results . . . . . . . . . . . . . . . . . . 163

7.2 Future outlook . . . . . . . . . . . . . . 166

$\begin{array}{ll}\text { Appendices } & 168\end{array}$

$\begin{array}{lr}\text { A Machine control code } & 171\end{array}$

A.1 Bath cryostat . . . . . . . . . . . . . . . . . . 171

A.1.1 Electronics set up instructions . . . . . . . . . . . 171

A.1.2 Python code . . . . . . . . . . . . . 172

A.1.3 Arduino code . . . . . . . . . . . . . . 177

A.2 In-situ electrical measurements Varian . . . . . . . . . . . . . 178

B Data analysis code $\quad 181$

B.1 Cryostat auto-batch . . . . . . . . . . . . . . . . . . 181

B.2 Simmons model . . . . . . . . . . . . . . . . . . . . . . 184

$\begin{array}{ll}\text { C Drawings } & 187\end{array}$ 


\section{Chapter 1}

\section{Introduction}

The rare earths consist of the elements from Lanthanum, through to Lutetium. These elements are the ones through which the $4 \mathrm{f}$ electron level is filled, with La having an empty $4 \mathrm{f}$ level and Lu having a full $4 \mathrm{f}$ level. The rare earths react with nitrogen to form rare earth nitrides (RENs).

Promises of a simple crystal structure and interesting electrical and magnetic properties drew interest to the RENs already in the 1970s. The early experiments focused on chemically synthesising crystals of the RENs. After the initial burst of experiments, the study of the RENs slowed due to problems with crystal impurities from the synthesis, oxidation of the RENs and other technological limitations [1]. In the early 2000s vacuum technology reached a sufficient level that it became possible to make highquality crystals as well as thin films of the RENs which led to a revival of the field [1].

The rare earth nitrides are a family of materials which offer the possibility of combining different magnetic and electrical properties in exciting ways. Many of the materials in the REN family have been shown to be ferromagnetic semiconductors [1-5]. The RENs are chemically and structurally similar which offers the possibility of integrating many different RENs in a single device, taking advantage of their rich magnetic behaviour [1]. Electronic devices have been made in the past utilising the RENs, such 
as spin filter Josephson junctions using $\mathrm{GdN}$ barriers, spin filter tunnel junctions with GdN barriers, magnetic tunnel junctions with DyN barriers, and magnetic tunnel junctions using $\mathrm{GdN}$ and $\mathrm{SmN}$ electrodes and AlN barriers [6-9].

A magnetic tunnel junction consists of two ferromagnetic layers separated by a thin insulating barrier layer. The resistance of the junction can be controlled by the relative magnetic orientation of the two ferromagnetic layers, where the resistance of the junction is low if the layers are magnetised parallel and high if the two layers are magnetised anti-parallel. The high and low resistance states of a magnetic tunnel junction are utilised in magnetic random access memory, where the high and low resistance states correspond to a 1 or a 0 in the computer.

The RENs are particularly well suited for magnetic tunnel junctions given their epitaxial compatibility within the series, and epitaxial compatibility with many III-V materials which may be used in the insulating barrier. The RENs is the only family of materials with many intrinsic ferromagnetic semiconductors. High spin polarisation is needed for high efficiency in an magnetic tunnel junction. The range of magnetic behaviour within in the RENs offer the possibility of tailoring the magnetic tunnel junction to a specific application.

This thesis aims to expand the work on magnetic tunnel junctions in Ref [9] by presenting devices using a different tunnel barrier material, show developments of new manufacturing methods, and a variety of experiments aimed at characterising $\mathrm{GdN}$ and $\mathrm{SmN}$ in more detail.

\subsection{Chapter outline}

Chapter 2 is a background to the rare earth nitrides (RENs). The chapter examines the structural, magnetic, and electrical properties of the rare earth nitrides, with a focus on properties that are relevant in subsequent chapters. 
Chapter 3 contains details on sample preparation, characterisation, and measurements relevant for subsequent chapters. The sample preparation sections include growth chamber details, rare earth nitride and capping layer growth, and photolithography and shadow lithography. The characterisation and measurement sections include in-situ and ex-situ electrical measurements, magnetic measurements as well as measurements of crystal structure.

Chapter 4 contains an investigation into the interaction between a rare earth surface and nitrogen. This chapter takes an experimental approach to study how the rare earth nitrides are formed, how nitrogen can move through a rare earth and rare earth nitride, and the time-scales involved in the formation of rare earth nitrides. The chapter finally demonstrates nitrogen being introduced into and removed from $\mathrm{SmN}$ thin films semireversibly.

Chapter 5 details considerations needed in the growth of gadolinium nitride $(\mathrm{GdN})$ and samarium nitride $(\mathrm{SmN})$ with a particular focus on how the growth needs to be managed in device manufacturing. The chapter starts with an investigation of how the $\mathrm{SmN}$ conductivity depends on the nitrogen pressure during growth. The chapter also shows how the conductance changes in $\mathrm{SmN}$ films as the films are deposited which can be used to understand what thickness ranges are of use in devices. The influence of ion exposure on GdN films is discussed in details with structural, magnetic, and electrical changes presented. Finally, the chapter presents the use of $\mathrm{Au}$ and $\mathrm{Al}$ for making ohmic contacts to REN films.

Chapter 6 describes magnetic tunnel junction devices manufactured using $\mathrm{GdN}$ and $\mathrm{SmN}$, with GaN tunnel barriers. We present devices with consistent behaviour between devices with the same structure thanks to a new device design. The chapter contains characterisations of the macroscopic structure of individual devices as well as the crystal structure of the REN films in the devices. The magnetic moment of the devices is measured to ensure they contain $\mathrm{GdN}$ and measure the coercive field for 
correlation with the switching field of the devices. The most extensive section of the chapter presents electrical characteristics for a number of devices. In the electrical characteristics section, we present measurements such as device consistency across a 2 inch wafer, current density-voltage $(\mathrm{J}-\mathrm{V})$ characteristics, and asymmetry in the J-V characteristics. The chapter also presents fits to the J-V characteristics using the standard Simmons model. Measurements of the tunnel magnetoresistance (TMR) and how this changes with temperature, applied field, current, and applied voltage are discussed. We investigate the switching of the devices and discuss the implication on the spin alignment of the electrodes. Finally, the chapter presents measurements of devices breaking and compares broken devices to functioning devices.

Chapter 7 summarises the entire thesis, concludes the scientific contribution of this thesis, and offer an outlook on the future industrial and scientific applications. 


\subsection{Publications}

1. W. F. Holmes-Hewett, F. H. Ullstad, B. J. Ruck, F. Natali, and H. J. Trodahl, "Anomalous Hall effect in SmN: Influence of orbital magnetism and 4f-band conduction", Physical Review B, vol. 98, p. 235201, 2018.

2. F. H. Ullstad, G. Bioletti, J. Chan, A. Proust, C. Bodin, B. J. Ruck, H. J. Trodahl, and F. Natali, “Breaking molecular nitrogen under mild conditions with an atomically clean lanthanide surface", ACS Omega, vol. 4, 5950-5954, 2019.

3. A. Shaib, F. Natali, J. R. Chan, F. H. Ullstad, W. F. Holmes-Hewett, J. D. Miller, B. J. Ruck, and H. J. Trodahl, "Coexisting structural phases in the catalytically driven growth of rock salt GdN", Submitted 2019.

4. J. D. Miller, F. H. Ullstad, H. J. Trodahl, B. J. Ruck, and F. Natali, “Vertical transport and tunnelling in rare earth nitride heterostructures", In preparation. 


\section{Chapter 2}

\section{The rare earth nitrides}

This chapter contains relevant background information to understand RENs and their properties in electronic devices, on which the research for this thesis is based. The first section presents a general crystal structure background before moving onto the structural properties of the RENs and what conclusions can be drawn from studying their structure. The second section deals with magnetism, and the magnetic properties of the RENs with a particular focus on $\mathrm{GdN}$ and $\mathrm{SmN}$. The third section presents electronic properties of the RENs, again with a particular focus on GdN and SmN.

\subsection{Structural properties}

Thin film growth is a complicated process, where the exact growth conditions of the growth control the resulting film structure $[10,11]$. There are many steps in forming a film. The resulting film properties can be influenced for example by substrate material choice and lattice parameter mismatch to growth species, growth species and growth rate, and substrate temperature. Some of the more common thin film growth modes include Frank-van der Merwe growth (layer-on-layer), Volmer-Weber growth (island growth), and Stranski-Krastanov growth (layer+island) [12]. In Frank- 
van der Merwe growth adatoms (atoms on the surface) preferentially attach to the substrate which promotes flat layer by layer growth. In VolmerWeber growth the interaction between adatoms is stronger than the interaction between adatoms and the substrate which promotes island formation [11]. The island growth is often modelled as hemispherical islands growing radially [13]. Stranski-Krastanov growth is a combination of the two previous growth modes, where the films grow as smooth layers until a certain critical thickness where further growth changes into islands [12].

Polycrystalline films are by definition composed of many grains, which is most easily described by the Volmer-Weber growth mode [14], and hence we will focus on this. When a thin film growth follows the Volmer-Weber growth mode, the progression can be illustrated as in fig. 2.1. Here the film growth can be seen from the side and the top. Initially, the film nucleates in multiple sites across the substrate. More adatoms will attach to the initially nucleated sites, with the rate of adatom attachment depending on the exact shape of the island. As the islands grow, there will eventually be a continuous path formed across the substrate by islands coalescing, called the percolation threshold. Once percolation is reached a current can generally be passed through the film. Once the islands coalesce to a full film, the faster-growing islands will outcompete the slower growing islands, and a dominant crystal orientation may emerge [15].

The way the atoms of the growing material stack on the substrate and each other will produce different crystal structures. The rare earth nitrides form the simplest crystal structure, fcc rocksalt structure, as seen in fig. 2.2 $[1,16,17]$. Rocksalt structure is a simple ABABAB stacking, where each layer of the crystal is identical to its next nearest neighbour plane.

In a perfect crystal, the REs are equal in number to the nitrogen in the crystal lattice, but vacant nitrogen sites in the lattice arising from nitrogen deficient growth conditions are common, and these vacancies are the main electronic dopants [1]. The large difference in electronegativity between the rare earth ion and nitrogen ion leads to the bonding between the two 


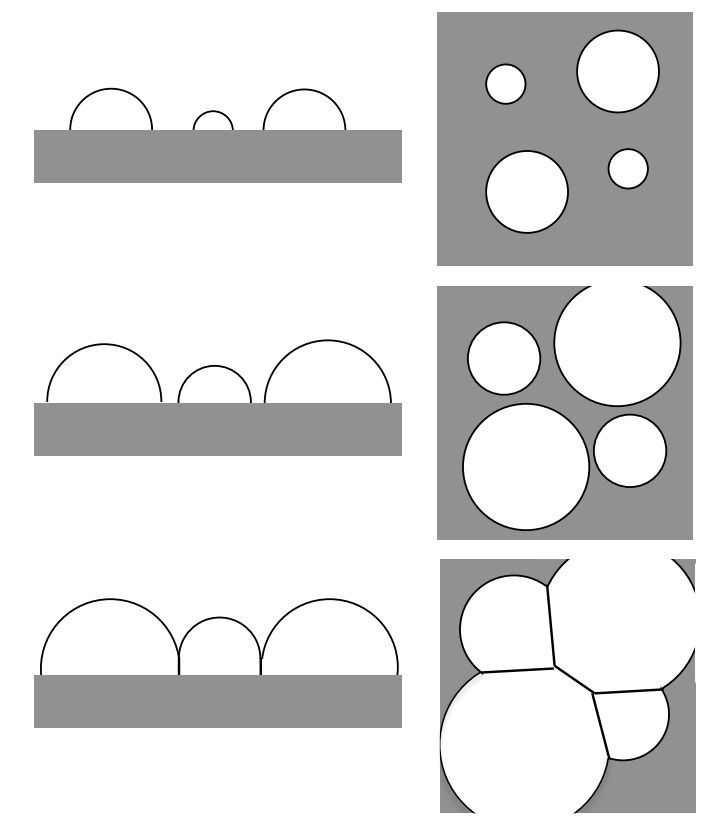

\section{Side view Top view}

Figure 2.1: Volmer-Weber island growth in side view and top view showing the islands growing and reaching percolation.

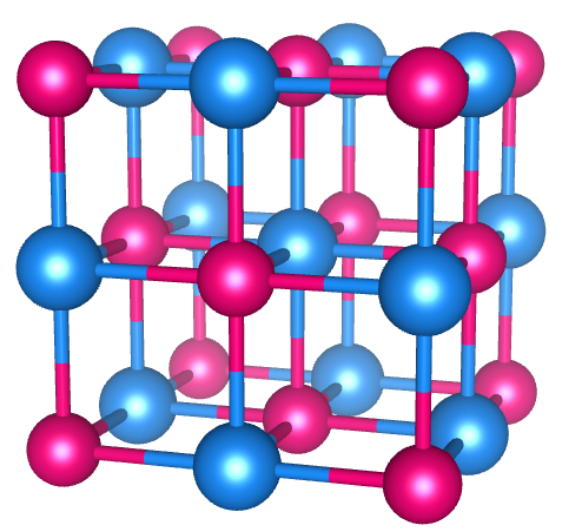

Figure 2.2: A REN rocksalt crystal. Pink - RE, Blue - N. 
being of predominantly ionic character [18].

The rare earth nitride thin films have been grown under a variety of conditions; using physical vapour deposition (PVD), molecular beam epitaxy (MBE), pulsed laser deposition (PLD), rf magnetron sputtering, and the nitrogen can be supplied using molecular nitrogen, nitrogen ions, nitrogen plasma, or ammonia [17, 19-22]. Differently prepared REN films appear to behave slightly differently, as evidenced by different groups finding some RENs to be metallic, while others find the same REN to be semiconducting [1].

The crystal structure of the REN is often studied using X-ray Diffraction (XRD), which measures the crystal orientation out of the film plane. In fig. 2.3 typical XRD patterns of two GdN films are shown, one epitaxial and one polycrystalline GdN film. The strongest GdN peak, corresponding to a (111) oriented surface, is visible around $31^{\circ}$. Around $65^{\circ}$ we see the reflection of the (111) peak as the GdN (222) peak. In polycrystalline growth, the RENs commonly adopt a preferential (111) orientation. The remaining strong peaks in fig. 2.3 are from the substrate. Comparing the room temperature polycrystalline GdN XRD pattern to the high temperature grown GdN sample in fig. 2.3 the epitaxial GdN has an increase in the magnitude of the GdN (111) and GdN (222) peaks relative to the substrate peaks.

Many things can be extracted from the XRD spectrum, such as the crystal structure, lattice parameter, and crystallite size.

The lattice parameter is the distance between atomic planes in the crystal. The lattice parameter, $d$, can be calculated from the position of the crystal peaks in the XRD spectrum using Bragg's law

$$
n \lambda=2 d \sin \theta
$$

where $n$ is an integer, $\lambda$ is the $\mathrm{X}$-ray wavelength, and $\theta$ is the angle of incidence relative to the plane of the film.

Across the rare earth nitride series, the lattice parameter varies from 


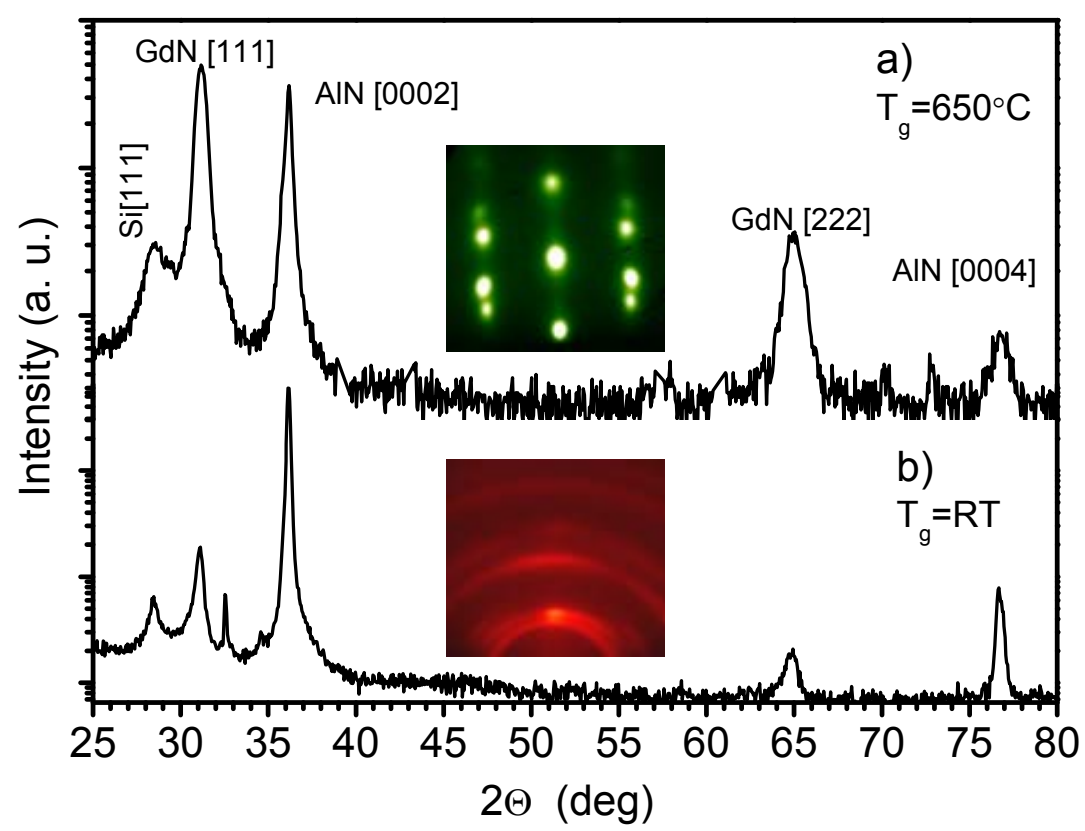

Figure 2.3: XRD sprectra of two GdN thin films deposited with the substrate at (a) $650{ }^{\circ} \mathrm{C}$ and at (b) room temperature. Reproduced from Ref [23] with permission.

$5.035 \AA$ (LaN) to $4.76 \AA(\mathrm{LuN})$ [1]. This corresponds to a lattice constant variation of $11 \%$ over the entire series. RENs closer together in the series have a smaller lattice mismatch, such as GdN (4.974 $\AA$ ) to $\mathrm{SmN}(5.035 \AA)$ which gives a lattice mismatch of around $1 \%$. The lattice constant decrease across the series arises from the increasing amount of $4 \mathrm{f}$ electrons which decreases the ionic radii of the rare earth anions [1].

The crystallite size, $\tau$, is calculated using the Scherrer formula, seen in eq. (2.2).

$$
\tau=\frac{K \lambda}{\beta \cos \theta}
$$

where $K$ is a dimensionless shape dependent factor (typically $K=0.9$ ), $\lambda$ is the wavelength of the $X$-ray source, $\beta$ is the line broadening at FWHM, and $\theta$ is the angle where the peak is centred. 
By studying the full width at half maximum (FWHM) of the diffraction peaks and using the Scherrer equation, it is possible to determine the average size of the crystallites making up the crystal orientation associated with said peak. The crystallite size in GdN and $\mathrm{SmN}$ is generally in the range of $10 \mathrm{~nm}$ to $20 \mathrm{~nm}$ for polycrystalline films and $>30 \mathrm{~nm}$ for epitaxial films [21, 24,25].

The RENs are sensitive to crystal impurities such as oxygen contamination during growth, but also sensitive to oxygen and water post-growth $[23,25,26]$. This sensitivity leads to the thin films needing to be protected by a capping layer before the films leave the ultra-high vacuum environment. The REN quickly turns into $\mathrm{RE}_{2} \mathrm{O}_{3}$ upon contact with the atmosphere $[27,28]$. Even just a visual inspection can be enough to determine if the REN film has oxidised, as the $\mathrm{RE}_{2} \mathrm{O}_{3}$ in severe cases forms a white powder that falls of the substrate - replacing the previously pristine thin REN film. XRD measurements confirm the presence of $\mathrm{RE}_{2} \mathrm{O}_{3}$ as an amorphous background which steals intensity from the ordered diffraction peaks.

\subsection{Magnetic properties}

A number of the rare earth nitrides are paramagnetic at room temperature, and ferromagnetic at cryogenic temperatures, with GdN having the highest ferromagnetic transition temperature of $70 \mathrm{~K}$ (depending on preparation conditions). In this section, we will discuss paramagnetism and ferromagnetism in general as well as the relevant magnetic properties of the RENs for future chapters of this thesis.

\subsubsection{General magnetism}

When a ferromagnetic material is heated enough that the thermal energy overcomes the exchange energy, it becomes paramagnetic. The material still orders magnetically but requires an external magnetic field to do so. 
The strength of the magnetisation in a paramagnetic material (M) is proportional to the applied field (B).

$$
\mathbf{M}=\chi \mathbf{B}
$$

The proportionality constant $\chi$ is the magnetic susceptibility, which is a measure of how strongly a material aligns with a magnetic field.

For a paramagnetic material, where the amount of aligned spins is small, and hence $\chi$ is small, the magnetic susceptibility is found to follow the Curie-Weiss law

$$
\chi=\frac{C}{T-T_{C}}
$$

where $C$ is the Curie constant, $\mathrm{T}$ is the temperature, and $T_{C}$ is the Curie temperature. As the temperature approaches $T_{C}$, the assumptions behind the Curie-Weiss law no longer hold as the susceptibility approaches infinity. Infinite $\chi$ means magnetic ordering can persist without an applied magnetic field - meaning the material has entered a ferromagnetic phase. Obviously, we cannot measure an infinite susceptibility, so getting a correct measurement of the Curie temperature can be tricky. Some common ways of finding $T_{C}$ include plotting $1 / \chi$ against temperature and fitting a straight line to the high-temperature data or plotting $\chi$ versus temperature in the ferromagnetic phase and fitting a Brillouin function [24,29].

To extract $T_{C}$ the temperature dependent magnetisation has to be measured. Two common measurements of the magnetisation of a ferromagnet using a QuantumDesign MPMS (Magneticurie tec Property Measurement System) are the zero-field-cooled (ZFC), and field cooled (FC) measurements of the sample. In a ZFC measurement, the sample is cooled to the lowest temperature without a field applied. When the sample is in the ferromagnetic state, having aligned the domains without an applied field, a field is applied. The sample is heated up in the applied field and as the temperature increases the magnetisation decreases. In an FC measurement, the sample is cooled in the measurement field from the maximum temperature which is to be measured. 


\subsubsection{Magnetism of GdN}

In fig. 2.4 the temperature dependent FC normalised magnetisation of three GdN films with different carrier concentrations has been reproduced from Ref [29]. The figure presents data from three GdN film with different carrier concentration (Low, Medium, High) and the magnetisation of the GdN film in the ferromagnetic state changes with the carrier concentration.

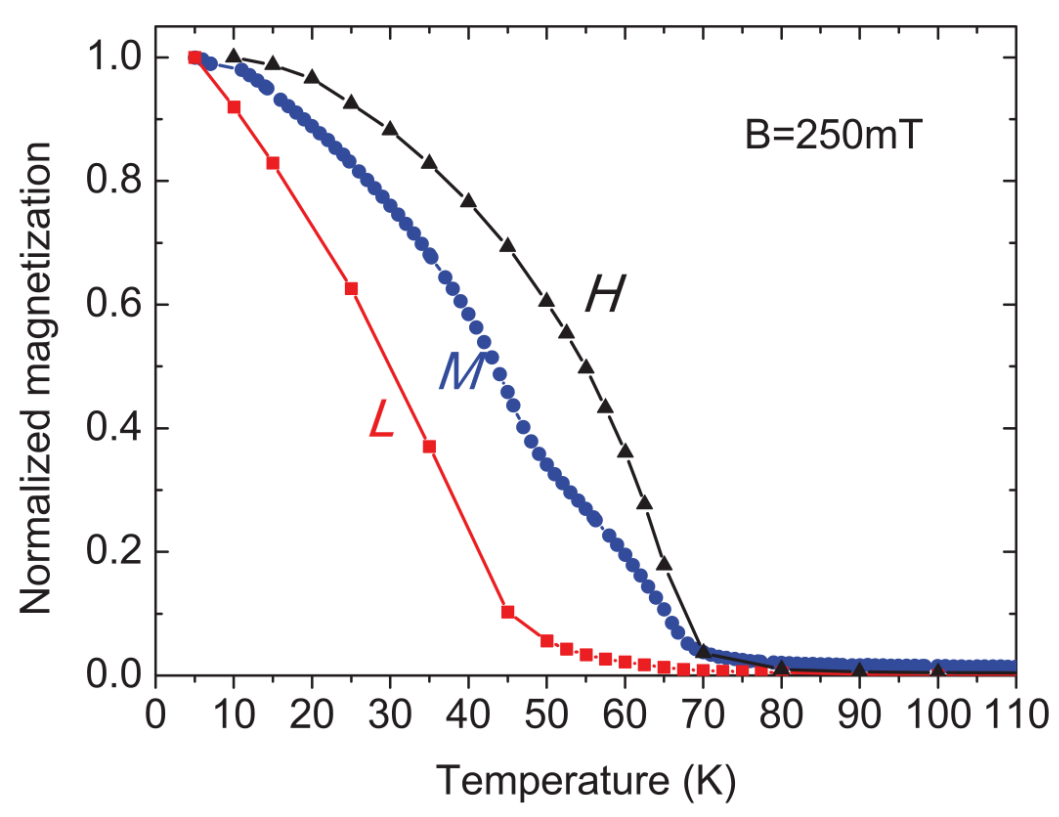

Figure 2.4: Temperature dependent field cooled magnetisation of three GdN films with different carrier concentrations (Low, Medium, High) showing slightly different shapes of the onset of the ferromagnetic phase. Reproduced from Ref [29] with permission.

The high carrier concentration $\mathrm{GdN}(\mathrm{H})$ film in fig. 2.4 has a sharp onset of ferromagnetic ordering at $70 \mathrm{~K}$. In contrast, the low carrier concentration GdN film (L) has a more gradual onset of ferromagnetic ordering around $50 \mathrm{~K}$. The GdN film with a medium carrier concentration (M) lies in between the high and low curves and has an extra feature visible 
between $50 \mathrm{~K}$ to $70 \mathrm{~K}$. In sample $\mathrm{M}$ only temperatures below $50 \mathrm{~K}$ show complete ferromagnetic ordering. The data is interpreted as the magnetic transition at $70 \mathrm{~K} \mathrm{~K}$ being mediated by magnetic polarons at the doping sites while the $50 \mathrm{~K}$ transition arises from the stoichiometric GdN [29].

The saturation magnetisation and the coercive field are illustrated in fig. 2.5 which show the magnetic moment of a GdN film in the ferromagnetic phase as the applied field is changed, reproduced from Ref [30].

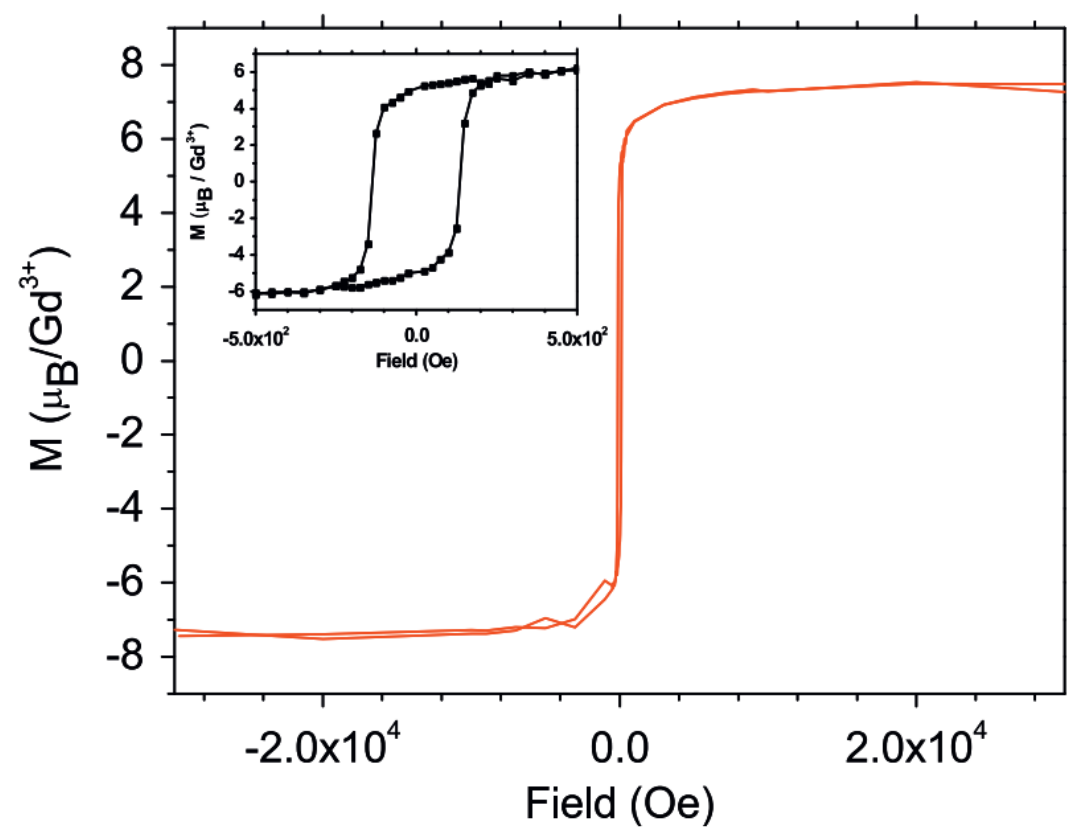

Figure 2.5: Magnetic hysteresis curve of a GdN thin film between $-3 \mathrm{~T}$ and $+3 \mathrm{~T}$ showing a saturated magnetisation of $7 \mu_{\mathrm{B}} / \mathrm{Gd}^{3+}$ ion. Inset: Magnification of low field behaviour showing a coercive field of $0.0125 \mathrm{~T}$. Reproduced from Ref [30] with permission.

The magnetic moment of the GdN thin film in fig. 2.5 rapidly saturates to $7 \mu_{\mathrm{B}} / \mathrm{Gd}^{3+}$ ion when a field is applied. The inset in fig. 2.5 shows a magnification of the low field behaviour which displays a coercive field, the field required to change the magnetic orientation, of $125 \mathrm{Oe}$ or $0.0125 \mathrm{~T}$. GdN has a small crystal anisotropy which leads to a small coercive field, 
which has been reported to be between $0.0010 \mathrm{~T}$ and $0.07 \mathrm{~T}$ depending on film properties and measurement temperature [21,31-33]. This small coercive field of GdN means the magnetisation direction is easy to flip, a useful property in a magnetic data storage device.

The magnetic moment of GdN arises only from the spin magnetic moment thanks to its half-filled $4 \mathrm{f}$ shell. The GdN magnetic moment magnitude is corroborated by multiple other sources in literature, both experimental and theoretical, as $7 \mu_{\mathrm{B}} / \mathrm{Gd}^{3+}$ ion in its ferromagnetic phase $[2,30]$. Some research also identifies an anti-ferromagnetic phase in $\mathrm{GdN}$ which is expanded $1.8 \%$ compared to the FM phase and enhances the coercive field by an order of magnitude [31]. Another study finds that a $2.4 \%$ expanded GdN lattice, prepared by ion-assisted growth as compared to $\mathrm{N}_{2}$ growth, is ferromagnetic but with a lower $T_{C}$ and a much higher magnetic field required to reach saturation magnetisation [34].

\subsubsection{Magnetism of $\mathrm{SmN}$}

Compared to GdN, SmN has two fewer electrons in the $4 \mathrm{f}$ shell which gives it an orbital contribution to the total moment. Local spin density approximation (LSDA+U) calculations show $\mathrm{SmN}$ as a low moment spin dominated ferromagnet [16]. Another theoretical study finds $\mathrm{SmN}$ to exist in both ferromagnetic and anti-ferromagnetic states [35]. These are at odds with experimental observations using X-ray Magnetic Circular Dichroism (XMCD) and calculations in Refs [24,36-38] where $\mathrm{SmN}$ is found to be a low moment orbital dominated ferromagnet.

In stark contrast to the large moment of $\mathrm{GdN}$, samarium nitride has the smallest ferromagnetic moment of the RENs of around $0.035 \mu_{\mathrm{B}} / \mathrm{Sm}^{3+}$ ion [24]. This low magnetic moment arises from a spin magnetic moment, opposed by a marginally larger orbital magnetic moment, giving a total moment that is near zero and orbital dominated. SmN has a large crystal anisotropy which coupled with this small magnetic moment creates a 
sizeable saturation field, more than $6 \mathrm{~T}$ at temperatures below $15 \mathrm{~K}$ with a coercive field of $0.9 \mathrm{~T}$ at $15 \mathrm{~K}$ and a coercive field of above $6 \mathrm{~T}$ at lower $\mathrm{T}$ [24].

Figure 2.6 shows the magnetic moment as a function of temperature for an SmN thin film, reproduced from Ref [24]. The magnetic moment is measured in $0.5 \mathrm{~T}$, and both the ZFC and FC measurements are displayed. The magnetic moment of $\mathrm{SmN}$ is orders of magnitude smaller than the GdN magnetic moment. The Curie temperature extracted from the peak in the ZFC curves of three SmN films is $27 \pm 3 \mathrm{~K}$ [24]. In the inset, the magnetic susceptibility rises rapidly as the temperature approaches $T_{C}$.

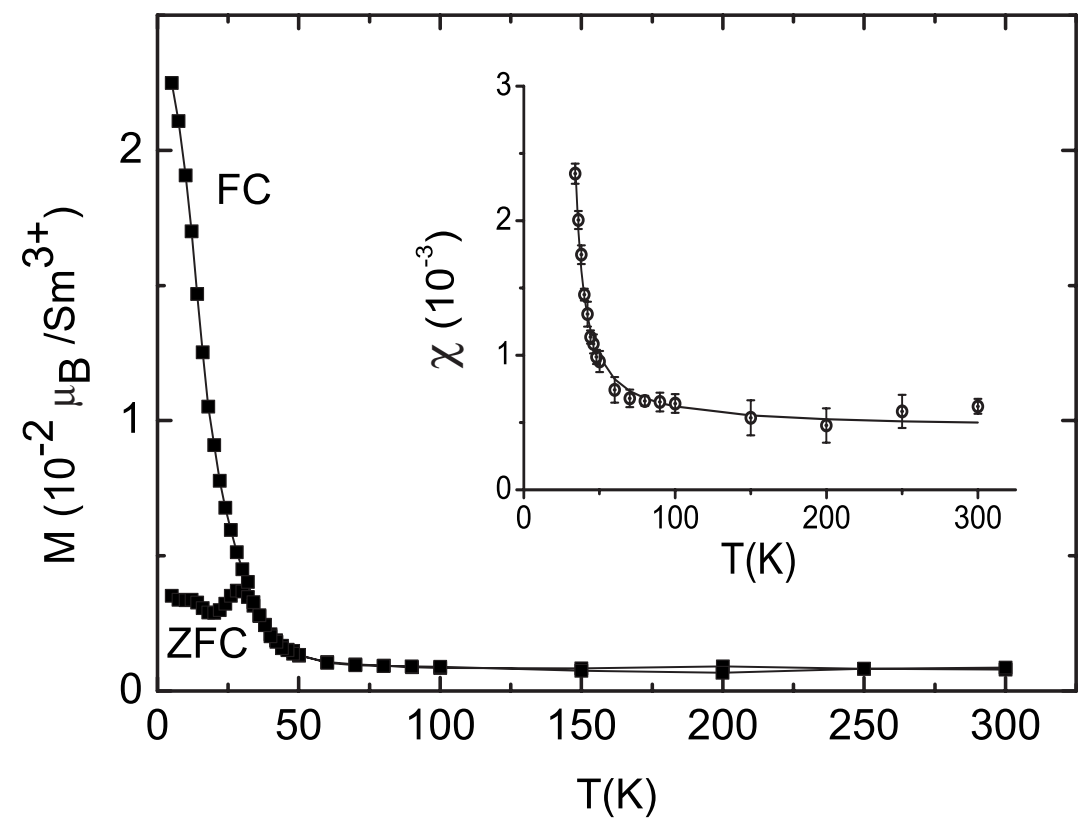

Figure 2.6: Temperature dependent magnetisation of $\mathrm{SmN}$ thin film measured in $0.5 \mathrm{~T}$. Inset: Temperature dependent magnetic susceptibility. Reproduced from Ref [24] with permission.

With the magnetic properties of $\mathrm{GdN}$ and $\mathrm{SmN}$ being in such stark contrast and being epitaxially compatible, superlattices of the two have been demonstrated [37]. In superlattices layers of the two materials are grown 
alternating with the intention of getting many interfaces between the two and hence a stronger interface effect signal which could offer insights in combining the two materials for device applications. XMCD measurements and calculations of the superlattices show a twist in the magnetic moment [37,38]. In fig. 2.7, reproduced from Ref [37], are two diagrams, with fig. 2.7 (a) showing the electron angular momentum directions in an applied field for a spin-dominant ferromagnet (GdN) and an orbital dominated ferromagnet $(\mathrm{SmN})$. In fig. 2.7 (b) the two materials have been brought into contact, and we see the propagation of a twisted magnetic phase through the $\mathrm{SmN}$ which originates at the GdN/SmN interface. The twist arises from a ferromagnetic exchange pinning of the spin magnetic moment of the SmN closest to the interface, which then relaxes to its free orientation further from the interface.

(a)

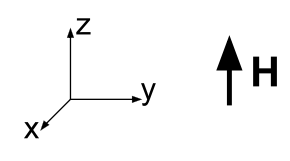

$\triangle$ spin moment \orbital moment

$\widehat{4}$ net moment

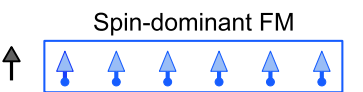

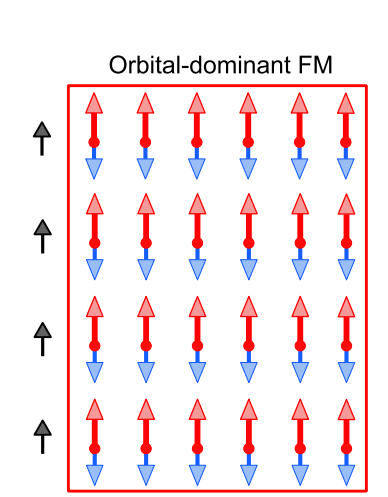

(b)

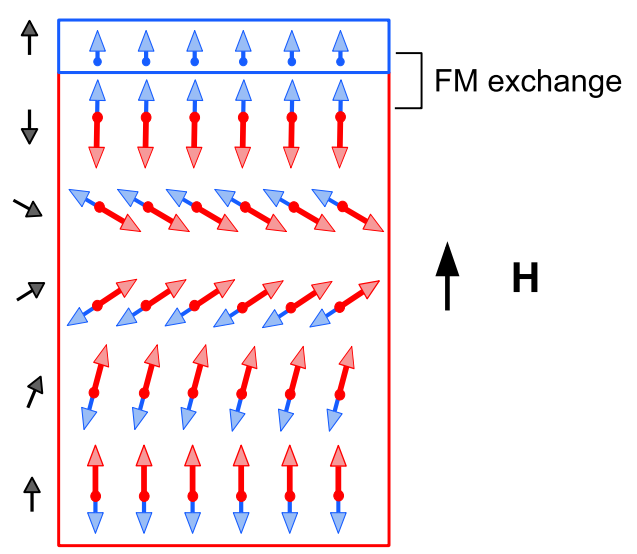

Figure 2.7: Diagram of the twisted magnetism phases induced in an orbital-dominant ferromagnet when brought into contact with a spindominant ferromagnet in an applied field. A ferromagnetic exchange at the interface pins the direction of the top layers of the orbital-dominant ferromagnet to the direction of the spin-dominant ferromagnet. The pinning introduces a magnetic twist in the orbital dominant ferromagnet. Reproduced from Ref [37] with permission. 


\subsection{Electrical properties}

This section will introduce the electrical quantities and properties which will be used in future chapters. We will start by defining basic electrical quantities. We then move on to discussing how the conductivity of the RENs can be tuned by changing the growth conditions. We then study the temperature dependent resistivity of $\mathrm{SmN}$ and GdN and the magnetoresistance of GdN.

The resistivity of a material, $\rho$, can be calculated as

$$
\rho=R \frac{A}{\ell}
$$

where $\mathrm{R}$ is the resistance of a uniform material, $A$ is the cross-sectional area of the material and $\ell$ is the length of the material. For this calculation to be meaningful, the material has to be homogenous, and the current density has to be uniform throughout the cross-sectional area. The resistivity is a material property; while the resistance is only a measured quantity, hence we compare the resistivity of thin films rather than the resistance.

This can be reformulated in terms of conductance in a thin film, as in eq. (2.6), where $G$ is the conductance, $\sigma$ the conductivity, $w$ the width of the film, $t$ is the thickness of the film, and $\ell$ the length of the conductor.

$$
G=\sigma \frac{w t}{\ell}
$$

Formulating the same concept in terms of conductance make it easier to explain phenomena using conductance contributions from different layers in a conductor.

The rare earth nitrides are sensitive to material composition, with the main dopant being nitrogen vacancies in the lattice [1]. Each vacancy can donate up to three electrons, as there is no nitrogen atom to accept the excess electrons from the trivalent rare earth ion, rendering the REN n-type doped [1]. The nitrogen-vacancy concentration can be changed by changing the nitrogen partial pressure in the chamber during film deposition [2]. 
This concentration change is exemplified in fig. 2.8, reproduced from Ref [2], where the in-plane conductivity of a GdN thin film is plotted against the nitrogen partial pressure during deposition. As expected from the nitrogen vacancies being the main dopants the conductivity increases when there is less nitrogen in the growth chamber. The pressure dependence of the conductivity is one of the main points of evidence for GdN being a semiconductor, rather than a metal or semi-metal, as this shows it is possible to dope the material. The corresponding results for $\mathrm{SmN}$ as prepared for this thesis will be presented in chapter 5 .

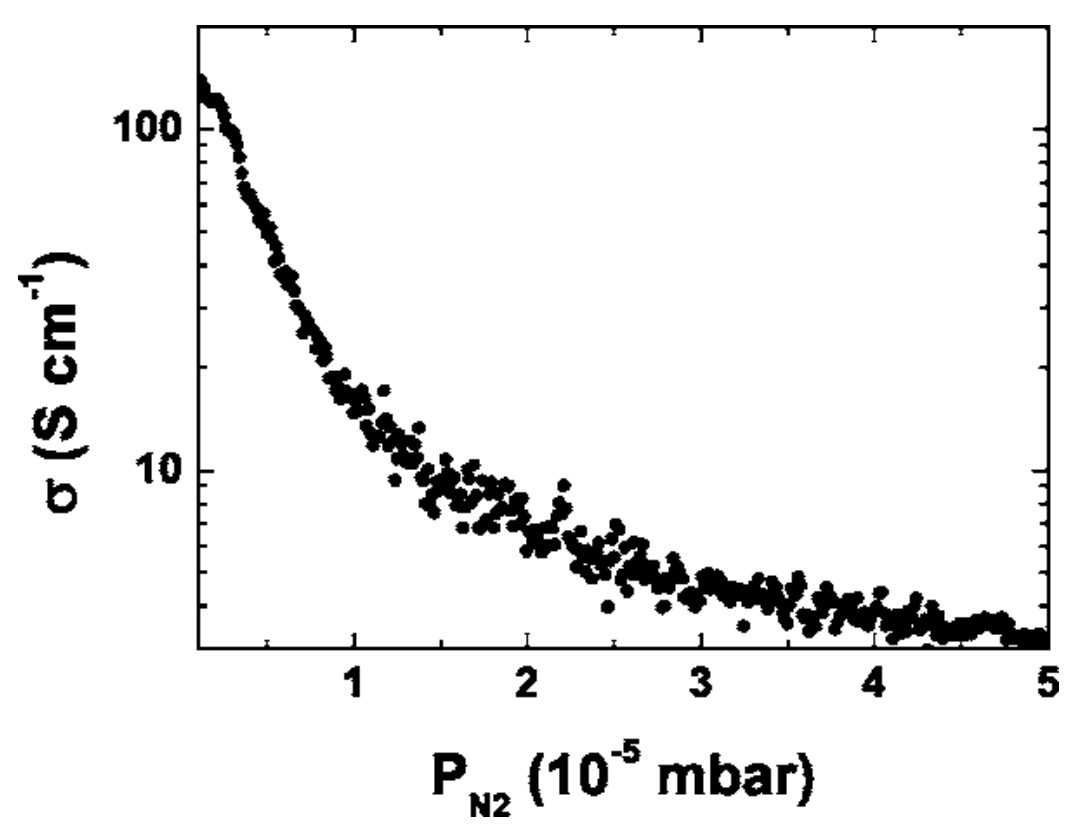

Figure 2.8: GdN conductivity vs nitrogen partial pressure measured insitu. The GdN conductivity is inversely proportional to the nitrogen pressure. Reproduced from Ref [2] with permission.

An important characterisation of the RENs is measuring the temperature dependent resistivity. GdN and $\mathrm{SmN}$ both have resistance anomalies associated with the onset of ferromagnetic ordering [29,36].

Figure 2.9, as reproduced from Ref [39], shows the temperature depen- 
dent resistivity for a single stoichiometric $\mathrm{SmN}$ film. The overall trend from this sample is a negative TCR (temperature coefficient of resistance), with the resistivity diverging at low temperature indicating a semiconducting SmN film. Centred around $30 \mathrm{~K}$ is a shoulder in the curve associated with the increased scattering as the $\mathrm{SmN}$ film becomes ferromagnetic. More doped SmN films can exhibit weaker negative TCR or even positive TCR. Heavily doped SmN samples can even be made superconducting [39].

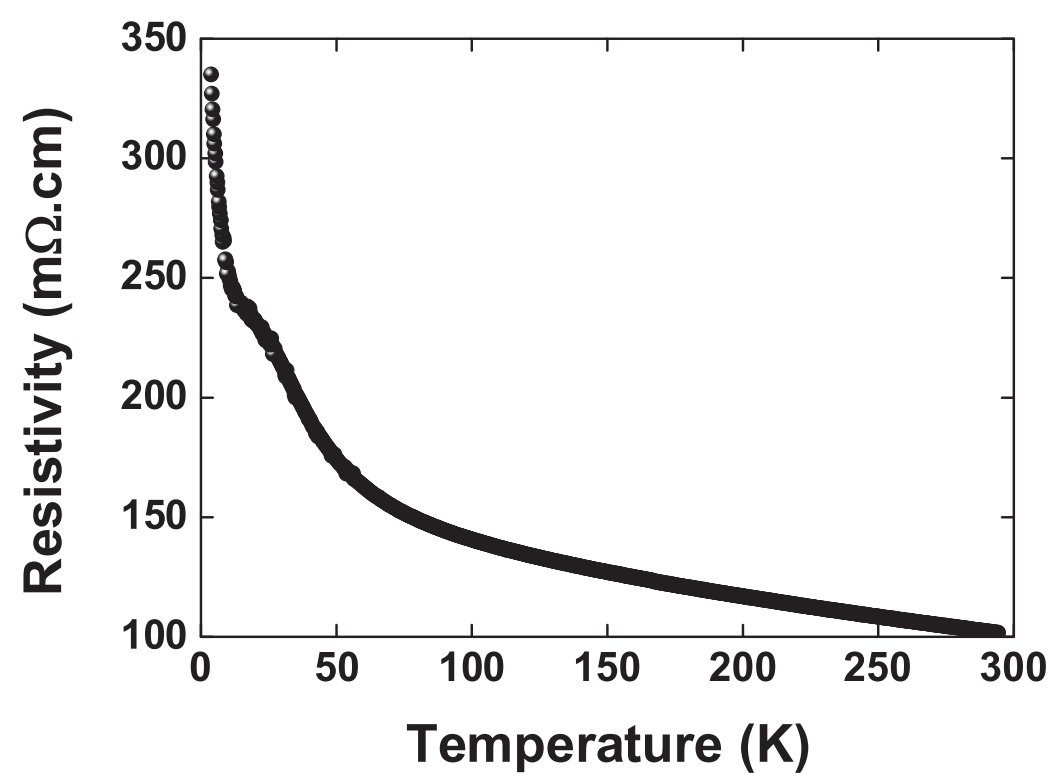

Figure 2.9: Temperature dependent resistivity of a stoichiometric $\mathrm{SmN}$ thin film showing a resistivity anomaly around $30 \mathrm{~K}$ associated with the SmN becoming ferromagnetic. Reproduced from Ref [39] with permission.

The corresponding temperature dependent resistivity of a similarly prepared single GdN film is shown in fig. 2.10, as reproduced from Ref [2]. Similarly to the SmN film in fig. 2.9 this GdN film also shows a negative TCR. There is a resistivity anomaly around $70 \mathrm{~K}$, again associated with the increased scattering as the film becomes ferromagnetic. The $T_{C}$ anomaly 
is $\mathrm{GdN}$ is often strong and can be correlated with magnetic ordering in the film [29].

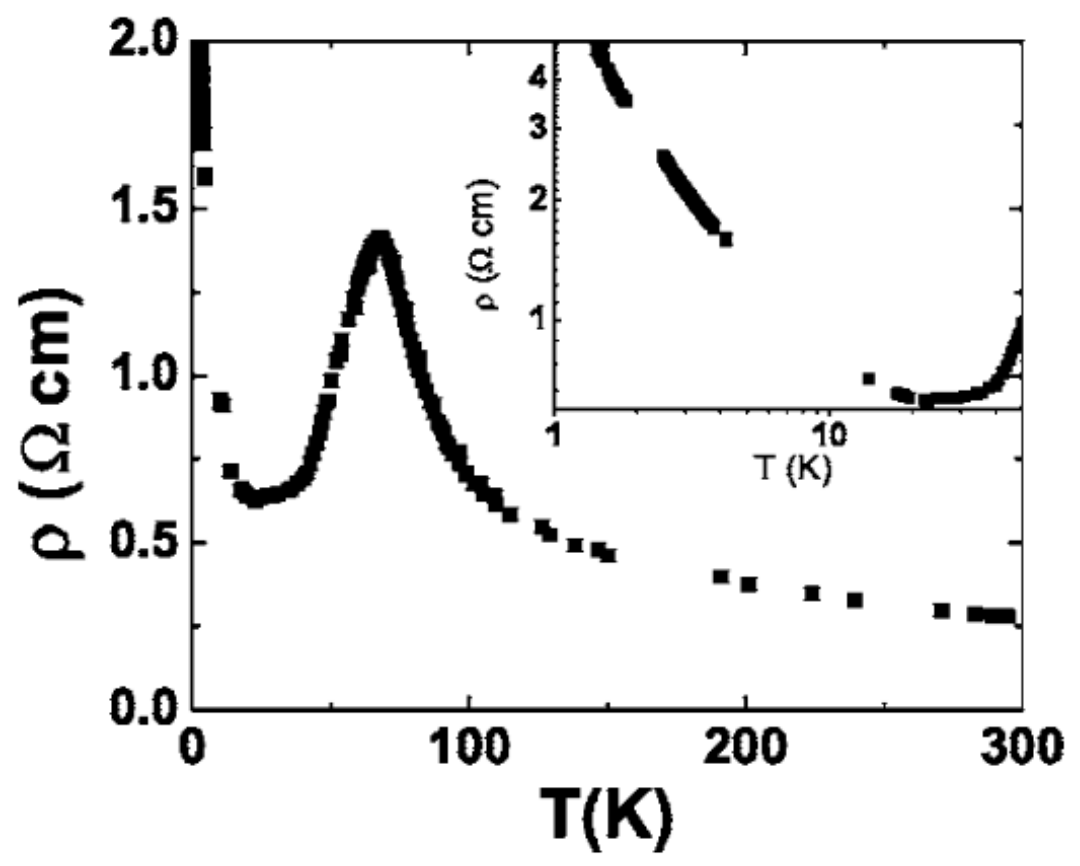

Figure 2.10: Temperature dependent resistivity of a GdN thin film showing a resistivity anomaly around $70 \mathrm{~K}$ associated with the $\mathrm{GdN}$ becoming ferromagnetic. Inset: Magnification of low temperature data on a log-log scale. Reproduced from Ref [2] with permission.

Not all GdN films show a negative TCR. Figure 2.11, as reproduced from Ref [40], show a GdN thin film prepared using reactive ion-beam sputtering. In the left-hand panel of fig. 2.11 the normalised resistance of this GdN thin film is plotted for different temperatures and applied fields. The GdN film has a low resistivity of $10 \mathrm{~m} \Omega \mathrm{cm}$ at room temperature. The TCR is negative above $T_{C}$ but positive below, but with the same $T_{C}$ anomaly as in fig. 2.10. The paper explains this TCR behaviour as a semiconductor-metal transition similar to that in EuO [40]. The hightemperature behaviour is the same for all applied fields. As the temper- 
ature drops below $200 \mathrm{~K}$, the $5 \mathrm{~T}(50 \mathrm{kOe})$ curve starts to diverge from the $0 \mathrm{kOe}$ and $1 \mathrm{~T}(10 \mathrm{kOe})$ curves. The $0 \mathrm{~T}$ and $10 \mathrm{~T}$ curves remain the same until around $70 \mathrm{~K}$. The $0 \mathrm{~T}$ resistance curve shows a large anomaly around $T_{C}$, but as the applied field is increased to $10 \mathrm{~T}$ and then to $50 \mathrm{~T}$, the anomaly becomes smaller.

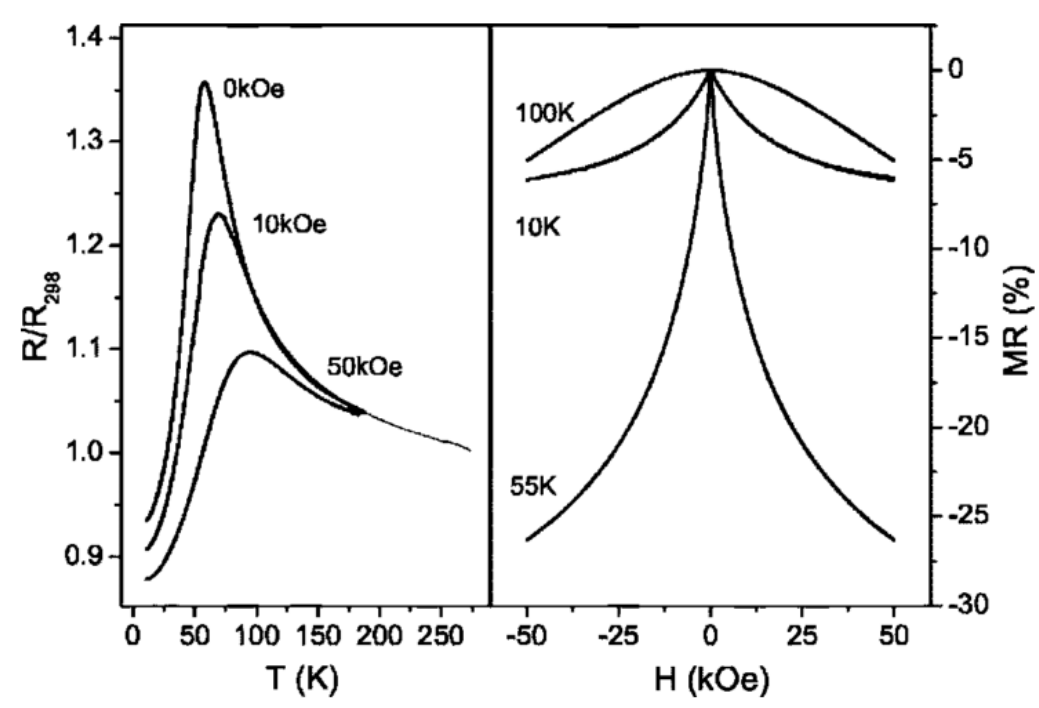

Figure 2.11: Left: Resistance vs temperaure for a GdN film under different applied fields. Right: Magnetoresistance of a GdN film at three temperatures (below, at, and above $T_{C}$ ). Reproduced from Ref [40] with permission.

In the right-hand panel of fig. 2.11 the magnetoresistance of a GdN thin film is plotted for different temperatures. The magnetoresistance is the change in resistance with an applied field. The $100 \mathrm{~K}$ curve which is well in the paramagnetic phase of GdN shows a rounded shape and a magnetoresistance of $\sim 5 \%$. As the temperature is decreased past the Curie temperature to $55 \mathrm{~K}$ the cusp-like magnetoresistance associated with the ferromagnetic phase becomes apparent, and the magnitude increases to $\sim 27 \%$. As the temperature is decreased further the cusp-like shape per- 
sists, but the magnitude of the magnetoresistance is decreased to $\sim 6 \%$. The maximum magnetoresistance magnitude of around $30 \%$ is consistent for GdN films in literature prepared under different conditions [21,40]. 


\section{Chapter 3}

\section{Experimental methods}

This chapter describes the methods and processes used to manufacture REN based thin films and electronic devices described in this thesis. Careful and accurate sample preparation is the key to reliable measurement data. The beginning sections deal with the ultra-high vacuum chamber preparation and operation, followed by basic parameters of REN film growth, and capping layer growth. Patterning techniques using photolithography and shadow lithography are then discussed, together with a presentation of the patterns used in device manufacturing for this thesis. Finally, the chapter ends with a description of the techniques used in electrical, magnetic, and structural measurements.

\subsection{Vacuum systems}

In order to make samples of high purity, the samples have to be manufactured in a chamber with a very low concentration of contaminants. An ultra-high vacuum (UHV) chamber is used for this purpose. A UHV chamber is a stainless steel enclosure where the pressure inside is kept below $1 \cdot 10^{-8} \mathrm{mbar}$. In the chamber, there are multiple ways of evaporating desired materials, for more on this see section 3.3. 
To keep the UHV chamber where samples are made as clean as possible, multiple vacuum chambers are usually connected in series. Higher vacuum is more easily achieved in a chamber for each step removed the chamber is from the atmosphere. Commonly the UHV system will have at least one load-lock chamber where samples will be loaded from atmospheric conditions. The load-lock is usually pumped down to $1 \cdot 10^{-7} \mathrm{mbar}$ before the sample is transferred to the next chamber. The next chamber is often the growth chamber, but for high purity systems, it is common to have a buffer chamber in between the load-lock and growth chambers where the pressure can be pumped down even lower before the gate valve to the growth chamber is opened.

\subsection{UHV systems at VUW}

The films grown in this thesis were grown in three different ultra-high vacuum systems, as described below.

Varian PVD system: This system has one electron beam source and two resistive evaporation sources (either for resistive boats or for a wire basket and crucible). It has an $\mathrm{N}_{2}$ gas connection and an ion source. The system comes apart to vent the entire chamber for loading new substrates and source materials. One growth takes at least one week as the system is fully vented between each growth, so the chamber and sources have to be baked and outgassed. The Varian system does not rotate samples during growth or control the substrate temperature. The film thickness is measured during the growth using a crystal balance deposition rate monitor. One of the main advantages of the system is the electrical feedthrough and in-situ electrical characterisation setup, which allows the resistance of a thin film to be measured during growth or for post-growth experiments.

The Varian system has been used to study the nitridation of rare earth films as well as the $\mathrm{SmN}$ conductivity dependence on $\mathrm{N}_{2}$ pressure. 
Thermionics MBE system: This UHV chamber has four electron beam sources and two resistive evaporation sources It has an $\mathrm{N}_{2}$ connection and a Kaufman EH200 ion source. The Thermionics system has a separate load lock chamber which allows samples to be loaded without venting the growth chamber to atmosphere. The system can heat and rotate samples as they are growing. The system also has a RHEED setup for monitoring the crystal structure during growth. The film thickness is measured using a crystal balance deposition rate monitor during the growth.

The Thermionics system has been used to grow a majority of the samples presented in this thesis including planar REN films, patterned GaN device components, and magnetic tunnel junctions.

Riber Compact 21 CLS MBE system: This UHV system has five effusion cells. It has an $\mathrm{N}_{2}$ connection and an Advanced Energy Cesar plasma source. The system has a separate load lock and a buffer chamber. Four substrates can be loaded into the load lock at once allowing quick sample turn-around times. The buffer chamber has a separate outgassing station to avoid contamination of the growth chamber. The system can heat and rotate samples during growth, thus also being capable of epitaxial growth. However, the sample holder is not actively cooled meaning the sample passively heats up during deposition. Most functions in the system are computer controlled and can be automated in recipes written in the manufacturer provided software. The system has a RHEED setup. There is a pyrometer for measuring the temperature of the heated sample during epitaxial growth. The Riber system does not have a direct way of measuring the thickness of the film during the growth, but it has a flux gauge which can be used to measure the atomic flux directed towards the sample.

The RIBER system has been used to grow magnetic tunnel junctions, and I developed a shadow mask system for making magnetic tunnel junctions without photoresist for use in the RIBER system. 


\subsubsection{Chamber maintenance}

To do maintenance inside the UHV system, it has to be opened up to the atmosphere. The maintenance involves turning off the high vacuum pumps, the roughing pumps, and then filling the chamber with a dry gas (often nitrogen or argon). This process is called venting, as the chamber is being vented to atmosphere. Depending on the required maintenance one or more flanges has to be removed. The flanges are then bolted back with a fresh copper gasket to create a high vacuum seal. The chamber is evacuated using first the roughing pump, and then the high vacuum pump.

Once the chamber is pumped out, all the flanges are tested for leaks using helium gas. If no leaks are found the chamber walls are slowly heated up to around $70{ }^{\circ} \mathrm{C}$ and maintained at that temperature for at least a week. Keeping the chamber at high temperature is known as baking the chamber. Baking is done to evaporate water and other contaminants from the walls of the UHV chamber and allow the pumps to remove it. While keeping the chamber walls hot, all source materials are heated up to their operating temperature for 1 hour or more. Some materials, such as samarium, will absorb much water and other contaminants in atmospheric conditions. These are important to get rid of before attempting to grow a sample with the source material. The process of removing the water and contaminants in the source materials is known as outgassing.

When the baking and outgassing are done, the chamber is left to cool down. A mass spectrometer is used to study the composition of the residual gas in the chamber. If the partial pressure of contaminants is low enough the chamber is considered clean.

To be able to grow reliable samples the growth process has to be calibrated after each maintenance cycle. The calibration process means every material that is grown in the UHV chamber has to be grown and characterised to ensure that they behave as expected. 


\subsection{Film growth}

While two of the UHV systems described above are capable of epitaxial REN growth, all samples in this thesis were grown at room temperature - resulting in polycrystalline films. The room temperature growth is necessary due to the photoresist patterning which can not be heated during growth. Thin films of samarium nitride and other materials were grown using Physical Vapour Deposition (PVD), resulting in multiple crystal orientations. A number of the rare earth nitrides, including $\mathrm{SmN}$ and $\mathrm{GdN}$, are easily grown by evaporating the rare earth in an atmosphere of molecular nitrogen. The rare earth atoms catalytically crack the molecular nitrogen to form the rare earth nitride. A REN is successfully formed at $\mathrm{N}_{2}$ partial pressures around $10^{-5}$ mbar to $10^{-4}$ mbar. The $\mathrm{N}_{2}$ :RE flux needs to be kept above 100:1 to form a nitride. The growth rate is generally $100 \mathrm{~nm} / \mathrm{h}$ to $400 \mathrm{~nm} / \mathrm{h}$.

A diagram (not to scale) of REN growth is seen in fig. 3.1 where the flux of a heated RE and the flux of $\mathrm{N}_{2}$ are directed towards a substrate where they form a thin film. In the growth chamber the $\mathrm{N}_{2}$ is introduced through a mass flow controller at the bottom of the chamber, approximately $500 \mathrm{~mm}$ from the sample holder. The $\mathrm{N}_{2}$ flux is directed towards the sample holder, but is assumed to be homogeneously distributed across the sample holder. The RE flux is directed from straight below (in the case of e-gun growth) and from $300 \mathrm{~mm}$ away at a $\sim 30^{\circ}$ angle from the normal. The sample holder is always rotated during growth to ensure a homogeneous film, where homogeneity was confirmed by XRD scans of different parts of the film. The REN forms not only on the substrate but also on the substrate holder and the chamber walls, but the REN deposited outside of the substrate is not used for measurements.

The rare earths can be evaporated in many ways, for example using electron guns, thermal sources, or sputtering systems. The nitrogen can be supplied as molecular nitrogen, atomic nitrogen, or ammonia. 


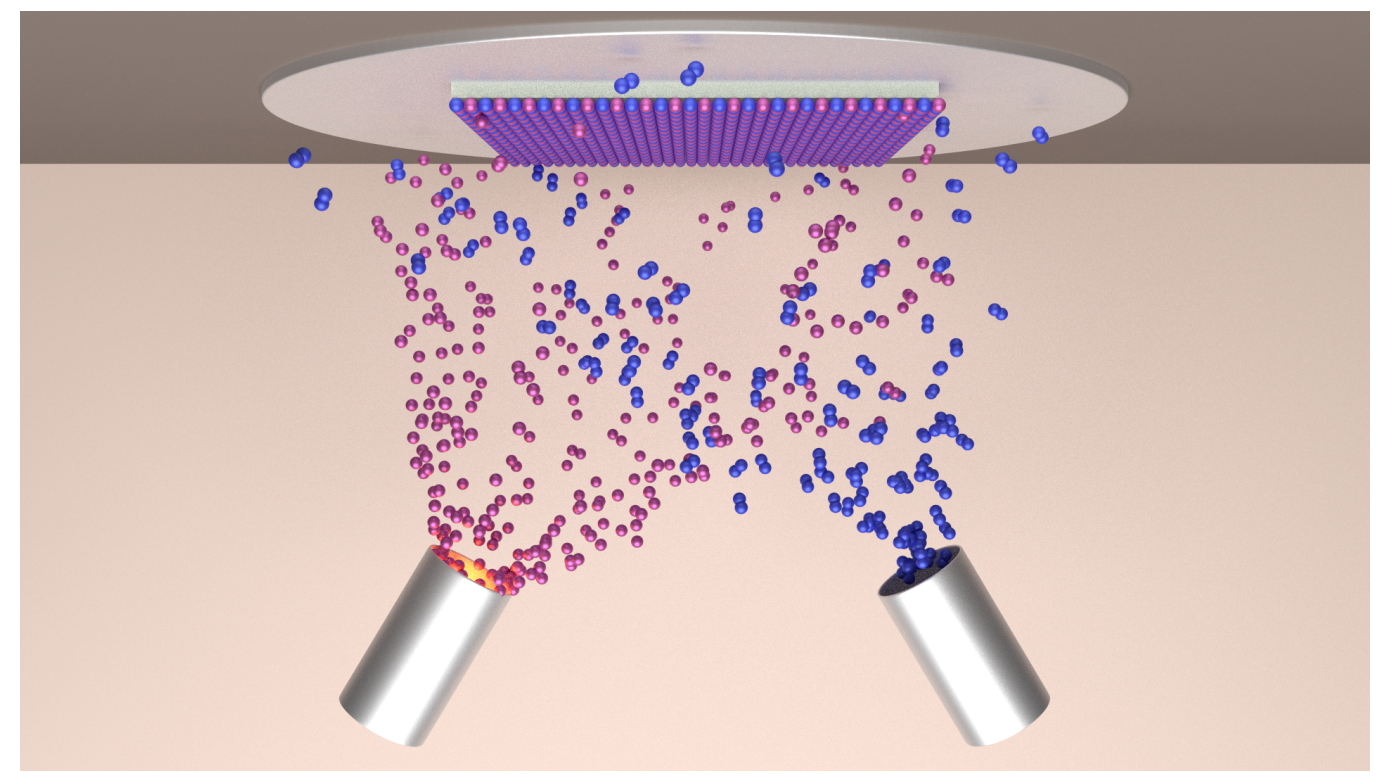

Figure 3.1: Growth of REN using heated RE (bottom left) and $\mathrm{N}_{2}$ (bottom right) onto a substrate (top). Not to scale.

\subsection{Capping layers}

Many of the rare earth nitrides oxidise easily upon contact with oxygen and water vapour, and care must be taken to protect the thin films against atmospheric conditions. The samples grown in this thesis were protected using a capping layer of similar thickness to the REN film. The capping material must not be grown using oxygen or hydrogen, as both of these react with the RENs.

When growing a resistive cap, often another nitride is used, for example, GaN or AlN. For uses where no nitrogen content is wanted, amorphous Ge or Si can be used to protect the sample.

When a conductive cap is desired, for example in measurements of the electrical transport perpendicular to the film plane, a metal is used. For samples grown for the preparation of this thesis $\mathrm{Sm}, \mathrm{Gd}, \mathrm{Nb}$, and $\mathrm{Al}$ have been used. 


\subsection{Patterned samples}

The two methods used for patterning samples for the research in this thesis are photolithography and shadow lithography. Patterning was done for making anything from pre-deposited electrical contact pads on small substrates (for use in in-situ electrical measurements) to making three-step patterns on 2 inch diameter substrates for magnetic tunnel junctions. Below we will describe the patterning of the MTJs. Photoresist is used for all patterned samples in this thesis, while the shadow lithography was developed and tested, but further progress was halted due to growth issues in the Riber MBE system.

\subsubsection{MTJ device pattern}

The pattern used for making the MTJ devices in this thesis is a three-step process where every step consists of photoresist patterning, film growth, and lift-off. For the MTJs this process was repeated three times to get the finished product, while for patterning done for simple contacts for other measurements a single photoresist patterning step was often sufficient.

In the first step in the device manufacturing, ten dumbbell-shaped bottom contacts are patterned across the substrate together with four rectangular pieces at the bottom, seen in fig. 3.2. Each of the ten bottom contacts has a number patterned next to it for easy identification of the finished devices. The rectangular pieces are used in for example structural and magnetic characterisation. On the wafer, there are four positional alignment markers towards the edges, and a single rotational alignment marker in the centre of the wafer which allows alignment to within $1 \mu \mathrm{m}$ between different pattern steps. In fig. 3.3 a single chip from the full wafer has been magnified to show more detail. Note that the in-plane and out-of-plane scales are not consistent in the images to make the structure more evident to the reader. A single chip measures $8 \mathrm{~mm} \times 9 \mathrm{~mm}$ at the outer edges of the bottom contacts while the thickness of the layers is on the order of 
$100 \mathrm{~nm}$.

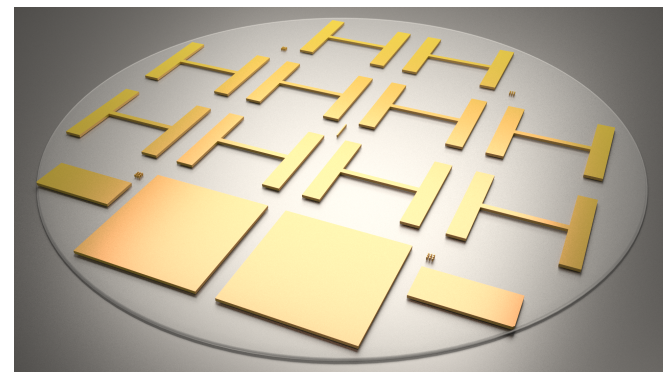

Figure 3.2: Full wafer pattern: step 1 - Gold bottom contacts.

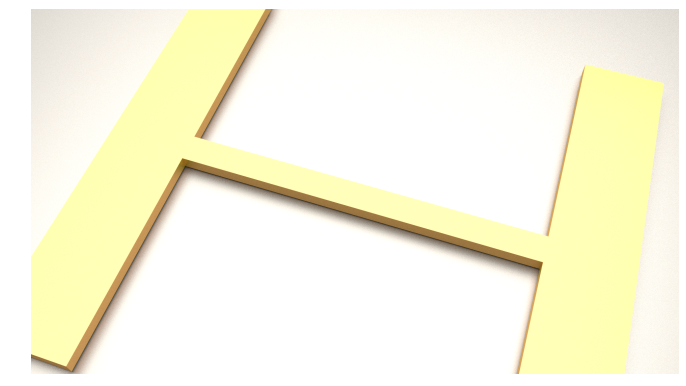

Figure 3.3: Single chip pattern: step 1 - Gold bottom contacts.

The second step in the device manufacturing requires patterning a layer which defines an area of each device through which the current flow. The layer will be referred to as the area restriction layer throughout the thesis. Figure 3.4 show the area restriction layer over the entire wafer, while fig. 3.5 shows a magnification of a single chip. The layer consists of $35 \mathrm{~nm}$ insulating GaN. The GaN covers the middle of the bottom contact except for two square windows, or active areas, through the GaN. The window allows direct contact in a well-defined area between the final layer and the bottom contact. Each chip has two square windows in the area restriction layer, and those windows have different side lengths so that one is always twice the side length (four times the area) of the other. The window side lengths range from $10 \mu \mathrm{m}$ to $200 \mu \mathrm{m}$ across the wafer. Most devices have different active areas, but there are three duplicate device sizes to be able to compare devices of the same area as well as study the effects of different areas. The edges of the bottom contact pads are also covered in $\mathrm{GaN}$ to remove the risk of short-circuiting a device if the patterns are misaligned. The insulating layer is chosen as GaN due to the propensity of the rare earth nitrides to form silicides or germanides when deposited on silicon or germanium. AlN was not used as the developer in the photolithography process reacts with $\mathrm{Al}$ and dissolves the AlN layer. 


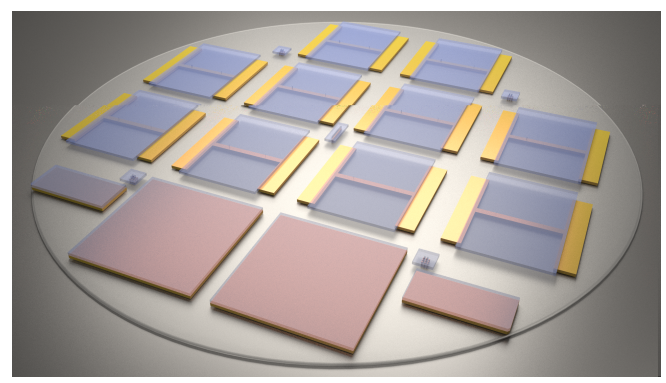

Figure 3.4: Full wafer pattern: step 2 - GaN area restriction layer.

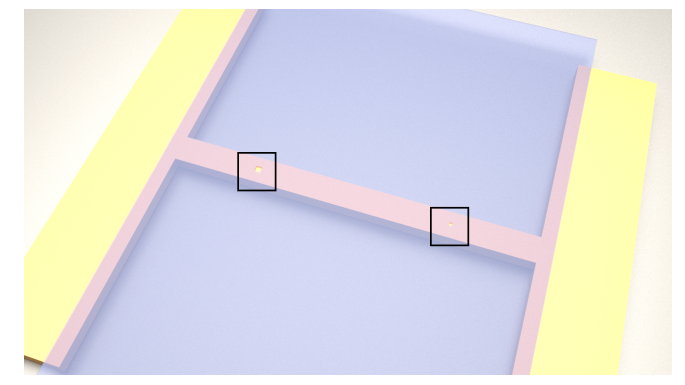

Figure 3.5: Single chip pattern: step 2 - GaN area restriction layer. The black squares mark the active area windows through the GaN.

The third and final step in the device patterning is the layer which contains one or more RENs and a capping layer. The final full wafer is shown in fig. 3.6, with a single chip magnification in fig. 3.7. The final step patterns two dumbbell-shaped devices on each device, perpendicular to the bottom contact. The waist of the REN layer dumbbell is $1 \mathrm{~mm}$ and thanks to the design of the area restriction layer the device manufacturing is robust up to a misalignment of $0.75 \mathrm{~mm}$, which is easily achievable even by eye and even easier with microscope assisted alignment using the alignment markers. The contact pads at the ends of the two device dumbbells are $2 \mathrm{~mm} \times 2 \mathrm{~mm}$, and they could not be made much smaller as devices are currently contacted by hand. The two devices on each chip were made different lengths to be able to easily identify which device is which on a particular chip as the active areas are different.

A cross-sectional schematic of the layers in the final device is shown in fig. 3.8. The REN stack in step 3 fills the active area, allowing direct contact between the Au bottom contact and the REN stack. The area restriction layer is grown thinner than the REN layers in the REN stack to ensure layer continuity across the active area edge.

This design could be used to scale to making even more devices per single bottom contact or chip. Two devices per chip give a large margin 


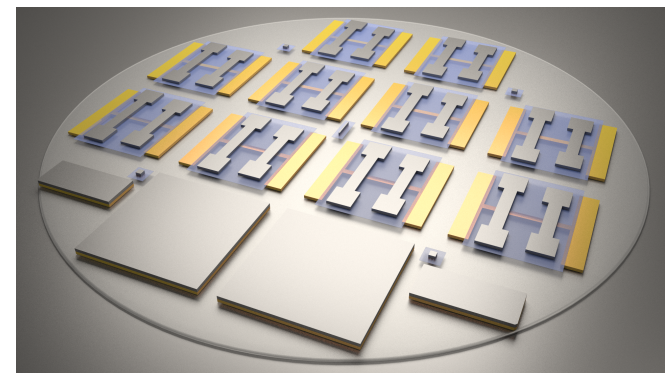

Figure 3.6: Full wafer pattern: step 3 - REN stack device layer.

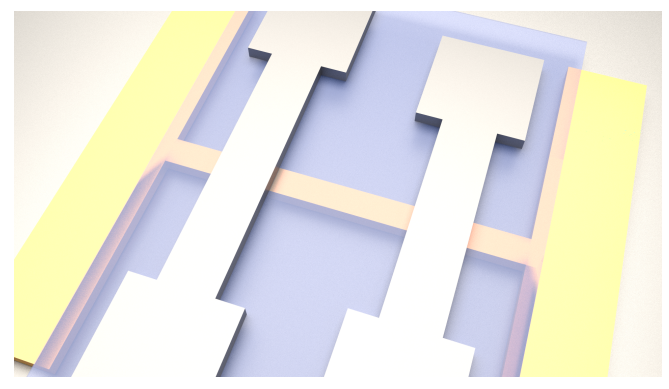

Figure 3.7: Single chip pattern: step 3 - REN stack device layer.

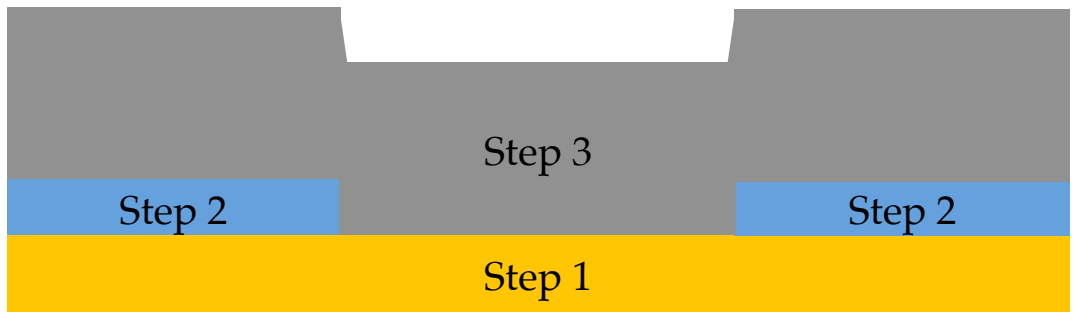

Figure 3.8: Final device structure over the active area in cross-section. Step 1: Au bottom contact. Step 2: GaN area restriction layer. Step 3: REN stack. Not to scale.

for potential misalignment as well as contact pads that are large enough to contact by hand. Most of our measurement setups can measure a maximum of two devices simultaneously, so cramming more devices onto the same chip is not necessarily more efficient than making more chips.

Drawings of the patterns can be found in appendix $\mathrm{C}$.

\subsubsection{Photolithography}

Photolithography is the process of using a photosensitive compound, called a photoresist, to selectively pattern a surface. Commonly this is done by first spin-coating a thin layer of photoresist onto a surface. The photoresistcovered surface is then covered by a physical mask, a photomask so that it can be selectively exposed to UV-light. The UV-light changes the struc- 
ture of the polymers in photoresist, and the light-exposed parts are easily washed off using a particular solvent, known as a developer.

In the research for this thesis multiple substrates, patterns, and photoresists have been used for various purposes. Photoresists used have been standard AZ1518 and AZ5214E, where the latter was used mainly for the magnetic tunnel junction manufacturing due to its ability to give better edge definition. Photomask patterns were designed in the program layoutEditor and manufactured either at University of Canterbury or Callaghan Innovation.

The patterning was done on a Karl Suss MJB3 Mask Aligner. Different substrate materials, shapes and sizes have been used. Initially, a large portion of patterned samples was done on $10 \mathrm{~mm} \times 10 \mathrm{~mm}$ square sapphire substrates, but towards the end of the $\mathrm{PhD}$, we moved to 2 inch full wafers of silicon and sapphire to minimise the handling and potential surface contamination that occurs when cutting the wafers to smaller sizes before growth.

The final recipe used for patterning well-defined device structures is:

1. Spin coat AZ5214E at $4000 \mathrm{rpm}$ for $60 \mathrm{~s}$.

2. Soft bake for $1 \mathrm{~min}$ at $95^{\circ} \mathrm{C}$.

3. UV expose the full wafer to UV light for $0.25 \mathrm{~s}$.

4. Hard bake for $2 \min$ at $110^{\circ} \mathrm{C}$.

5. UV expose for $12 \mathrm{~s}$ seconds through a patterned photomask.

6. Develop in 4:1 351:B developer for $20 \mathrm{~s}$ seconds.

7. Double rinse in deionised water and blow dry with nitrogen.

8. Store in dust protected container in ambient conditions for up to two months. 
This process was developed by Dr Leo Browning for his PhD work.

The patterned substrates were loaded in various growth chambers and used to pattern metal contacts, insulating protection layers, and rare earth nitride-containing layer. To lift-off the photoresist to reveal the pattern, the samples were put in room temperature acetone for $10 \mathrm{~min}$ to $60 \mathrm{~min}$. Occasionally samples were sonicated for 1 min to remove photoresist on edges.

Photoresist-patterned samples grown in the Riber system, which lacks active sample cooling, were often hardbaked. To lift off the pattern the samples required multiple hours of sonication in $60^{\circ} \mathrm{C}$ NMP. This process severely degraded films and pattern integrity, so to get around this a shadow lithography process was developed.

\subsubsection{Shadow lithography}

Shadow lithography involves the use of a stencil to create a pattern on a substrate, by physically blocking the material from depositing in certain spots on the substrate. This technique allows patterning features of various sizes without the use of photoresist on the substrate. Being able to pattern without photoresist is useful in high-temperature film growth, as well as in applications where the cleanliness of the substrate is vital.

The development of shadow lithography capabilities for this research arose from necessity. The Riber MBE system does not have active sample cooling during growth which hard baked the photoresist and made liftoff practically impossible. However, the use of the shadow lithography as the last patterning step practically eliminates the risk of photoresist residue on the samples which is important for high device yield. Using shadow lithography also makes epitaxial growth of patterned samples a possibility. Unfortunately, growth issues in the Riber system prevented use of the shadow lithography system for this thesis, but the development is nonetheless interesting and will be of use for future research. 
The shadow mask and assembly to hold the substrate was designed in Fusion360. The shadow mask was manufactured by Veco Precision Metal, using $25 \mu \mathrm{m}$ thick Hard Nickel sheet. The mounting assembly was manufactured in the university mechanical workshop. The mounting assembly includes a stainless steel backplate which centres a pre-patterned 2 inch wafer in the holder. The $25 \mu \mathrm{m}$ shadow mask is put on top. A holding frame is bolted on top of the shadow mask to ensure good contact between the mask and the substrate as well as taking the strain off the thin shadow mask. These parts are all shown in fig. 3.9.

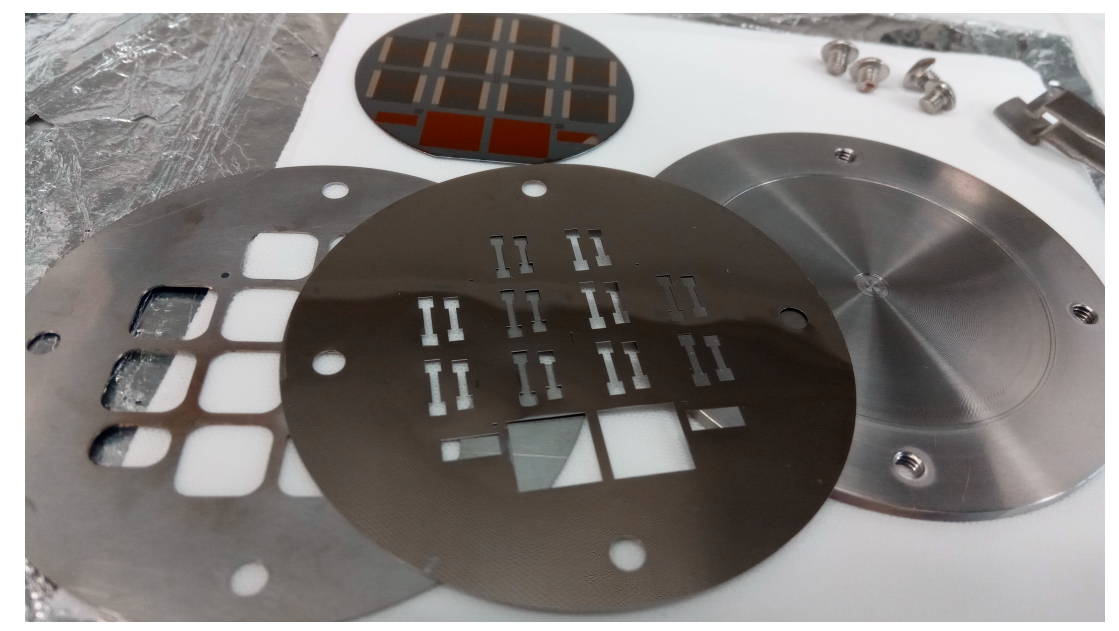

Figure 3.9: Shadow mask assembly. Left to right: Holding frame, shadow mask, back plate. Top: Pre-patterned substrate (gold bottom contacts and $\mathrm{GaN}$ area restrictors) and mounting bolts.

To grow a sample using the shadow mask the pre-patterned substrate needs to be loaded and centred by hand in the mask assembly. There are many alignment markers in the mask, but the patterns were designed to allow a $0.75 \mathrm{~mm}$ displacement error which is easily overcome by alignment by eye.

The shadow mask grows well-patterned films, and one is shown in fig. 3.11. The sample pattern edges are not as sharp as the edges from photolithography, but they appear to be sharp enough not to have issues 


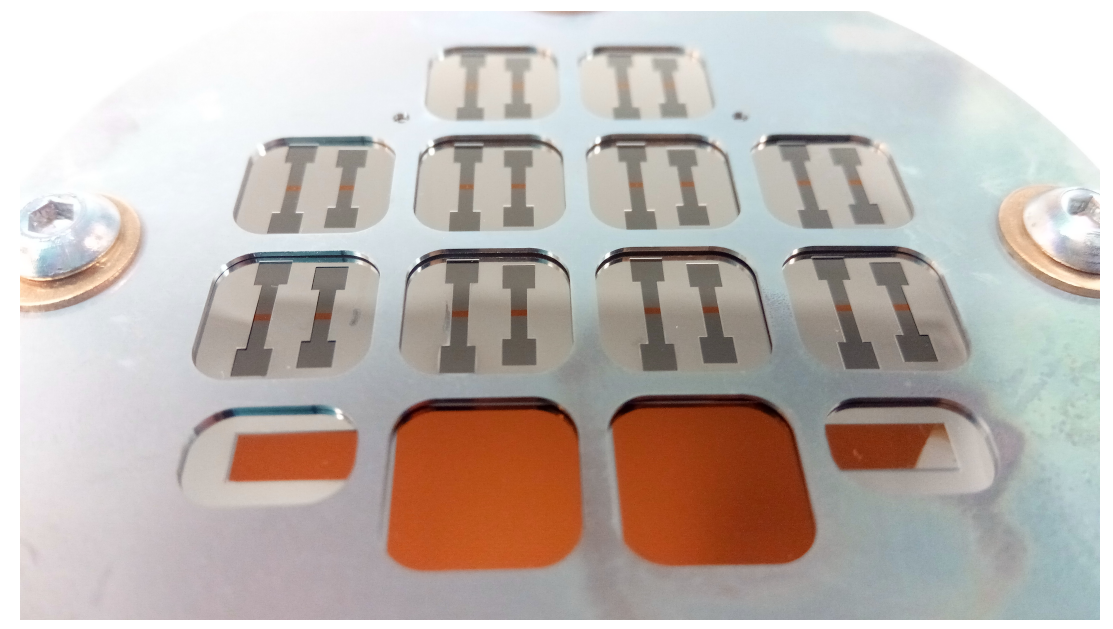

Figure 3.10: Shadow mask assembly assembled ready for growth.

with compromising layer integrity.

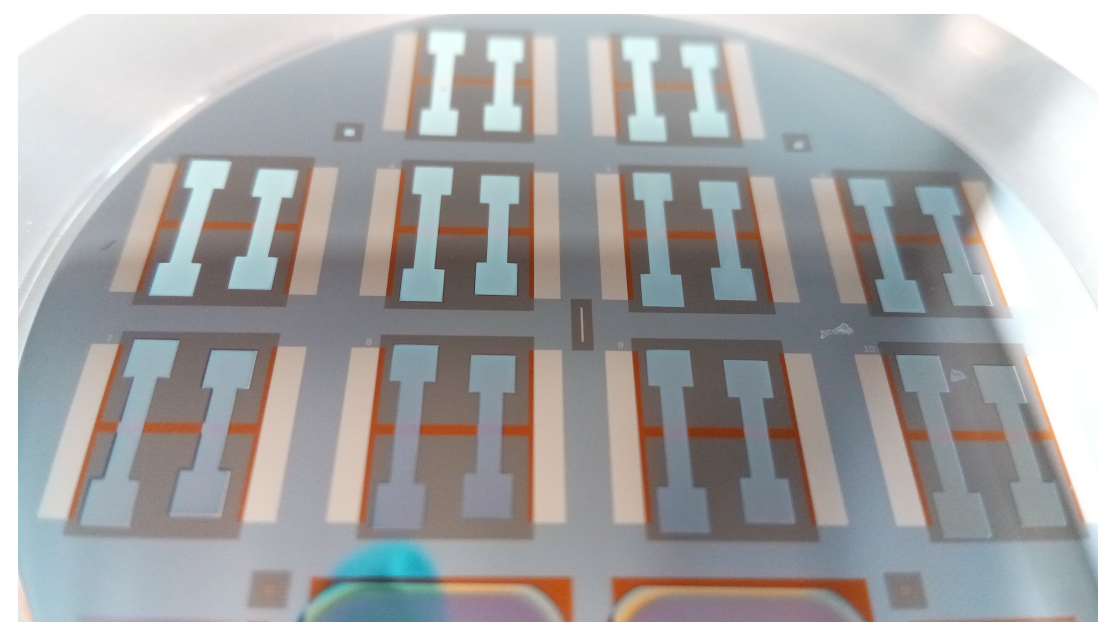

Figure 3.11: Finished patterned device after growth using shadow mask.

The surface profile along a line across the shadow-masked sample is shown in fig. 3.12. The baseline on either side of the main feature is the $\mathrm{GaN}$ area restriction layer. The two steep slopes on either side are the shadow edges, while the $35 \mathrm{~nm}$ dip in the middle shows the active area window depression propagated through the REN layers. The image shows 
the edges of the shadow mask area giving a smooth transition from the masked to the unmasked area. The shadow mask is $25 \mu \mathrm{m}$ thick, and this region of thickness increase should be around $25 \mu \mathrm{m}$ wide given the growth geometry, which corresponds well to the actual width of the edge region.

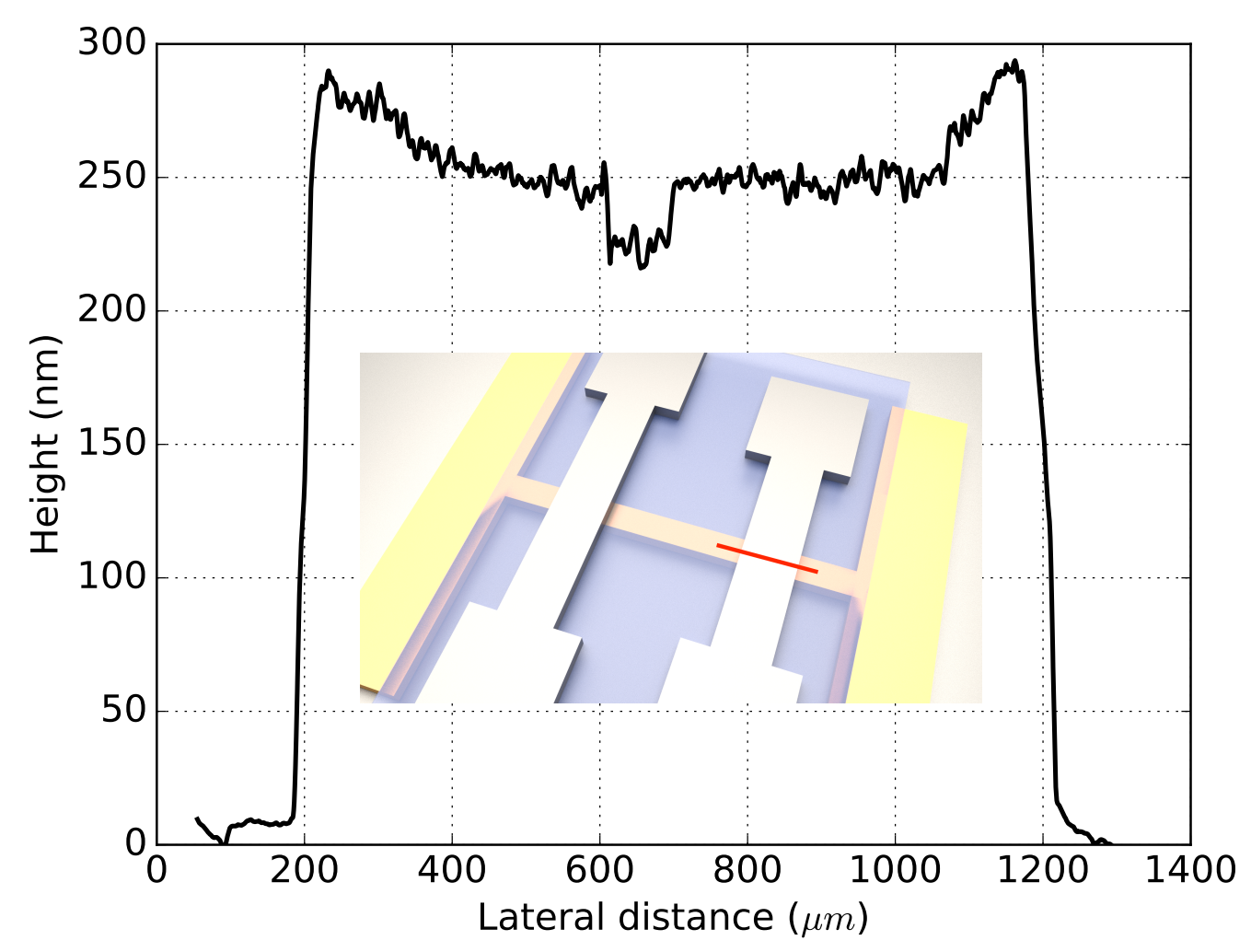

Figure 3.12: Surface profile of the stack in a MTJ grown using a shadow mask. The surface profile is measured using a Dektak profilometer along the red line in the schematic in the inset. The dip in the middle of the surface profile is where the stack is grown on top of the active area window.

The shadow mask setup was used to grow three patterned samples. However, due to issues with growing tunnel barriers in the Riber MBE system, which the mask was designed for, no magnetic tunnel junctions grown using the shadow mask showed the desired device characteristics. 
Once the growth of tunnel barriers has been sorted out in the Riber system, this method for growing patterned samples can be explored further and potentially be expanded to epitaxial device manufacturing.

Mechanical drawings of the shadow mask, as well as the support structure, can be found in appendix $C$.

\subsection{Characterisation and measurements}

This section describes characterisation and measurement setups and methods which will be used in this thesis, from which results will be shown in subsequent chapters.

\subsubsection{In-situ electrical measurements}

The Varian PVD system allows for in-situ electrical measurements using an electrical vacuum feed-through. The feed-through allows some electrical studies to be conducted on film growth and non-capped rare earth nitride films. The measurements are limited to a 2-point setup due to the design of the sample mount and electrical vacuum feed-through.

The electrical measurements are done by a Tektronix DMM4040 multimeter in auto-ranging resistance mode, which is connected to a Dell laptop for data-logging. The laptop also logs the chamber pressure read by the Pfieffer full range pressure gauge. The data was collected using a Python script written by myself, utilising an open source Pfeiffer pressure gauge interfacing package. The Python script can be found in appendix A. Data was sampled every second - a limit set by the communication speed of the multimeter via the ethernet connection. Thickness and growth rate, as measured by a crystal balance, was recorded manually.

Typically, a highly resistive sapphire substrate with two pre-deposited $2 \mathrm{~mm} \times 2 \mathrm{~mm} \mathrm{Cr} / \mathrm{Au}$ electrodes spaced $8 \mathrm{~mm}$ apart was attached to the sample stage of the UHV chamber. The $\mathrm{Cr} / \mathrm{Au}$ electrodes were patterned 
using photolithography and the $\mathrm{Cr} / \mathrm{Au}$ deposited in a thermal evaporator. The sides of the sample were masked off and held down using vacuum compatible Kapton tape. Masking the sides off ensures a well-defined current path between the electrodes, simplifying calculations. Copper wires were attached to the gold electrodes using molten indium, and the copper wires were connected to an electrical feed-through mount on the sample holder. The feed-through wires connected to the outside of the chamber where standard unshielded BNC cables were used to connect to the multimeter. Additional non-patterned substrates were also loaded onto the sample stage, such as sapphire and silicon, for $e x$-situ measurements (XRD, SEM). A diagram of the experiment pre-deposition is shown in fig. 3.13.

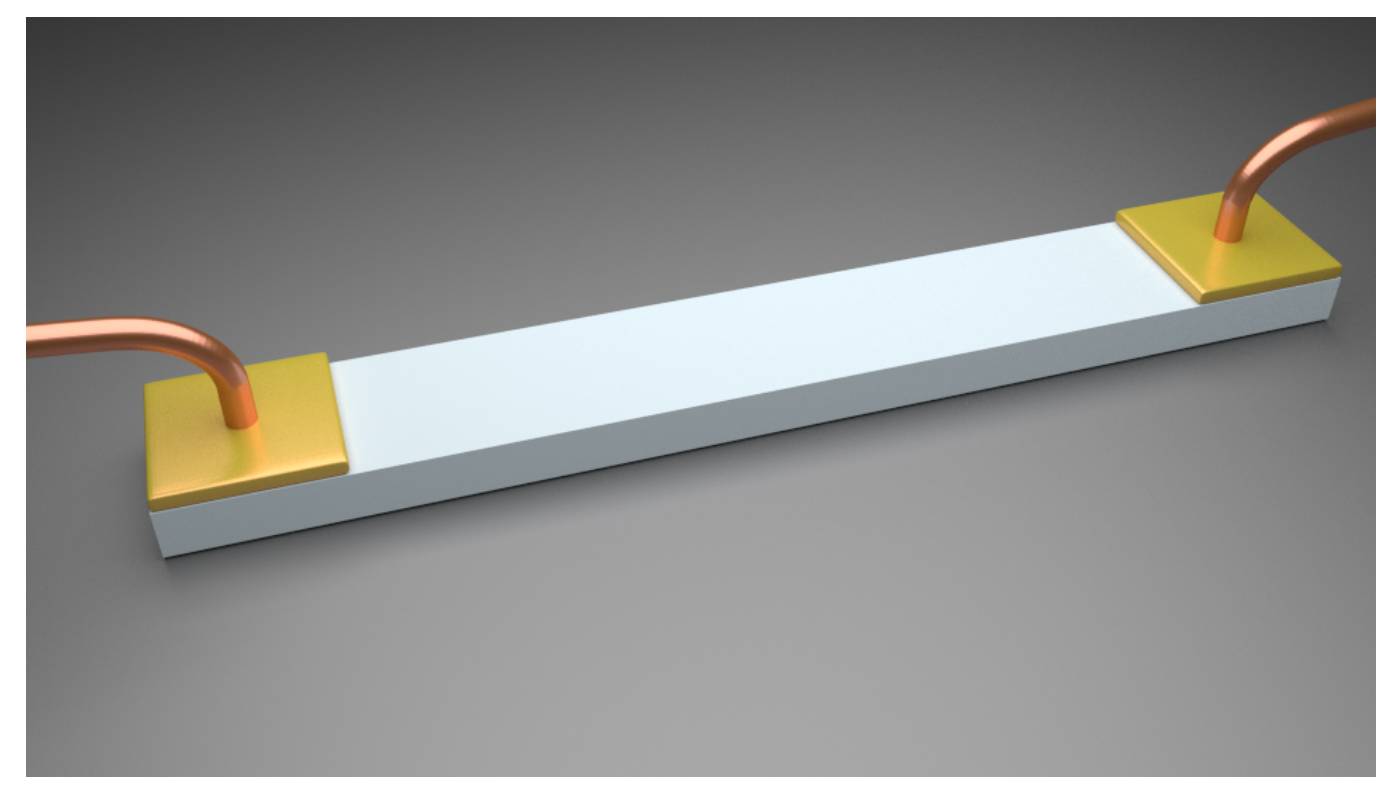

Figure 3.13: In-situ electrical measurement setup. Predeposited contacts on the edges of the substrate were contacted using molten indium and a wire passing through an electrical feed-through.

For each growth, the UHV chamber was vented, and a new substrate was loaded into the UHV chamber. The chamber which allows these electrical measurements to be made has no load-lock so loading a sample re- 
quires venting of the entire growth chamber. The sample was mounted on the sample stage, the sample stage was loaded, the electrical feed-through was connected, and a resistance check of all possible current paths was done. A shutter was moved into place to protect the samples until growth conditions were obtained.

Once the sample was loaded and set up correctly the chamber was pumped out using a roughing pump and a turbo pump. After the initial pump-down, the outgassing routine was initiated. The entire chamber was heated to above $70^{\circ} \mathrm{C}$ for at least seven days to bake out water and other impurities on the chamber walls. Each material source inside the UHV chamber was then heated up to operating temperature while continuing to bake the chamber walls. The heating of sources was done to minimise contaminants such as water, hydrocarbons, and oxygen from the material sources. Once the chamber and sources had been thoroughly outgassed the chamber was allowed to cool down for $24 \mathrm{~h}$ before an experiment started.

\subsubsection{Ex-situ electrical \& magnetic measurements}

Samples were electrically and magnetically characterised using three different setups. The Janis research closed cycle cryostat, the QuantumDesign Physical Property Measurement System (PPMS), and the QuantumDesign Magnetic Property Measurement System (MPMS).

\section{Janis research closed cycle cryostat}

The Janis research closed cycle cryostat SHI- $4 \mathrm{H}$ is cooled using a Sumitomo heavy industries F-50 helium compressor. The sample is mounted on a copper cold finger, and the temperature is regulated using an RDK coldhead, controlled by a Lakeshore 340 temperature controller. The electronics consist of a Keithley 224 programmable current source, a Keithley 182 Sensitive digital voltmeter, and a Keithley 196 System DMM multi- 
meter. The setup is controlled using a LabView program. Common measurements using this setup include the resistance of a sample at different currents and temperatures as well as current-voltage characteristics.

Samples are mounted using Apiezon N-grease onto a copper cold finger. Samples are pressed down to ensure good thermal contact with the stage. Electrical contact is made to the sample using copper wires which are stuck to the sample using electrically conductive silver paint (RS Pro). A photo of this can be seen in fig. 3.14.

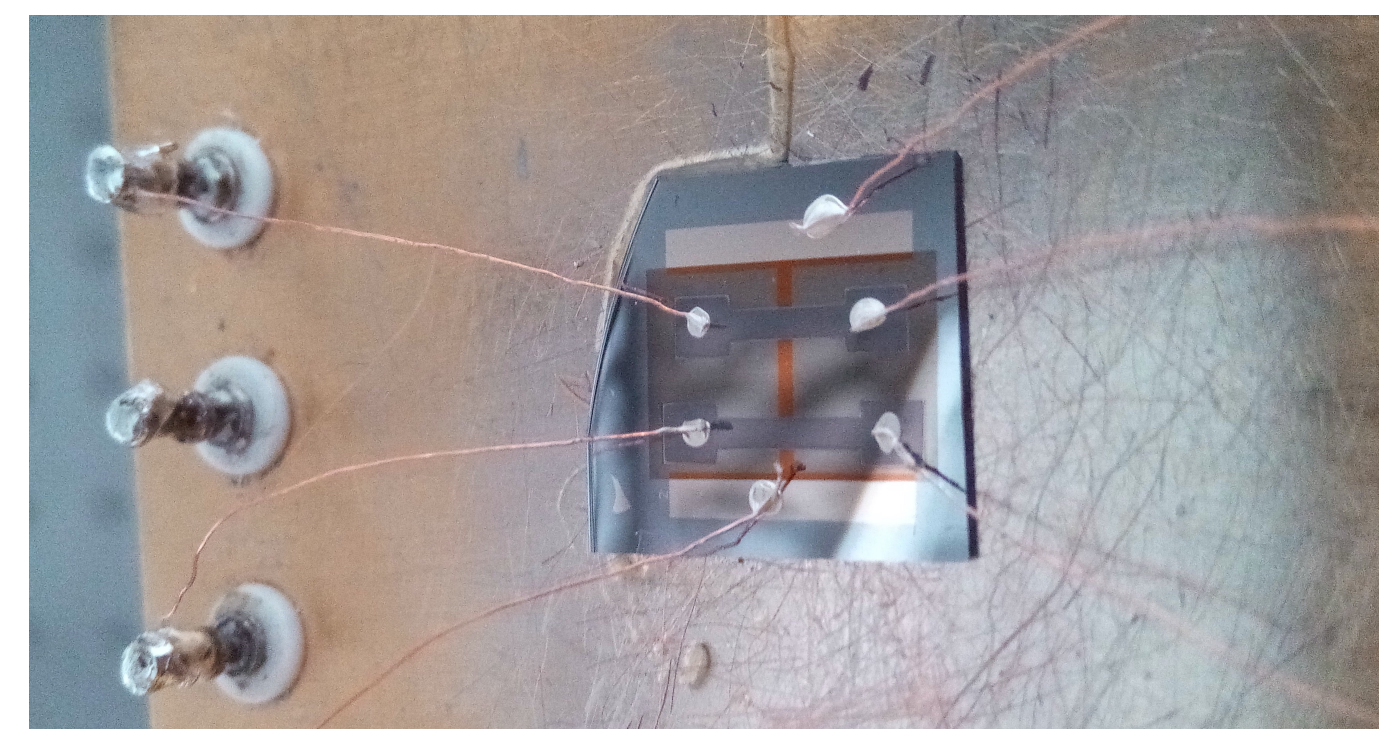

Figure 3.14: A sample mounted for electrical measurements in the Janis research closed cycle cryostat using Apiezon $\mathrm{N}$-grease, silver paint and copper wires attached to mounting posts. This sample has two devices mounted for sequential measurementes in the cryostat.

\section{QuantumDesign Physical Property Measurement System}

QuantumDesign Physical Property Measurement System (PPMS) is a machine capable of measuring many things relating to electrical and magnetic properties, with many measurement setup options being possible. The 
primary measurement option used in this thesis is the resistivity option with the rotator rod sample mount. This combination allows samples to be rotated and measured in a magnetic field. The sample resistance should be between $4 \mu \Omega$ and $4 \mathrm{M} \Omega$ for optimal performance. The machine is limited to measuring a maximum of $95 \mathrm{mV}$, which is not ideal for measuring tunnel devices but adequate for most other measurements. The temperature range is $2 \mathrm{~K}$ to $400 \mathrm{~K}$, and the field can be set and swept between $0 \mathrm{~T}$ and $\pm 9 \mathrm{~T}$, which means we can study, for example, the magnetoresistance and tunnel magnetoresistance of samples, measure the Hall effect, and other characteristics.

In fig. 3.15 we see a sample mounted on the PPMS mounting pucks. The samples are limited to maximum $10 \mathrm{~mm} \times 12 \mathrm{~mm}$. The sample is attached to the puck using GE-varnish and electrically contacted by hand using copper wires and silver paint. 


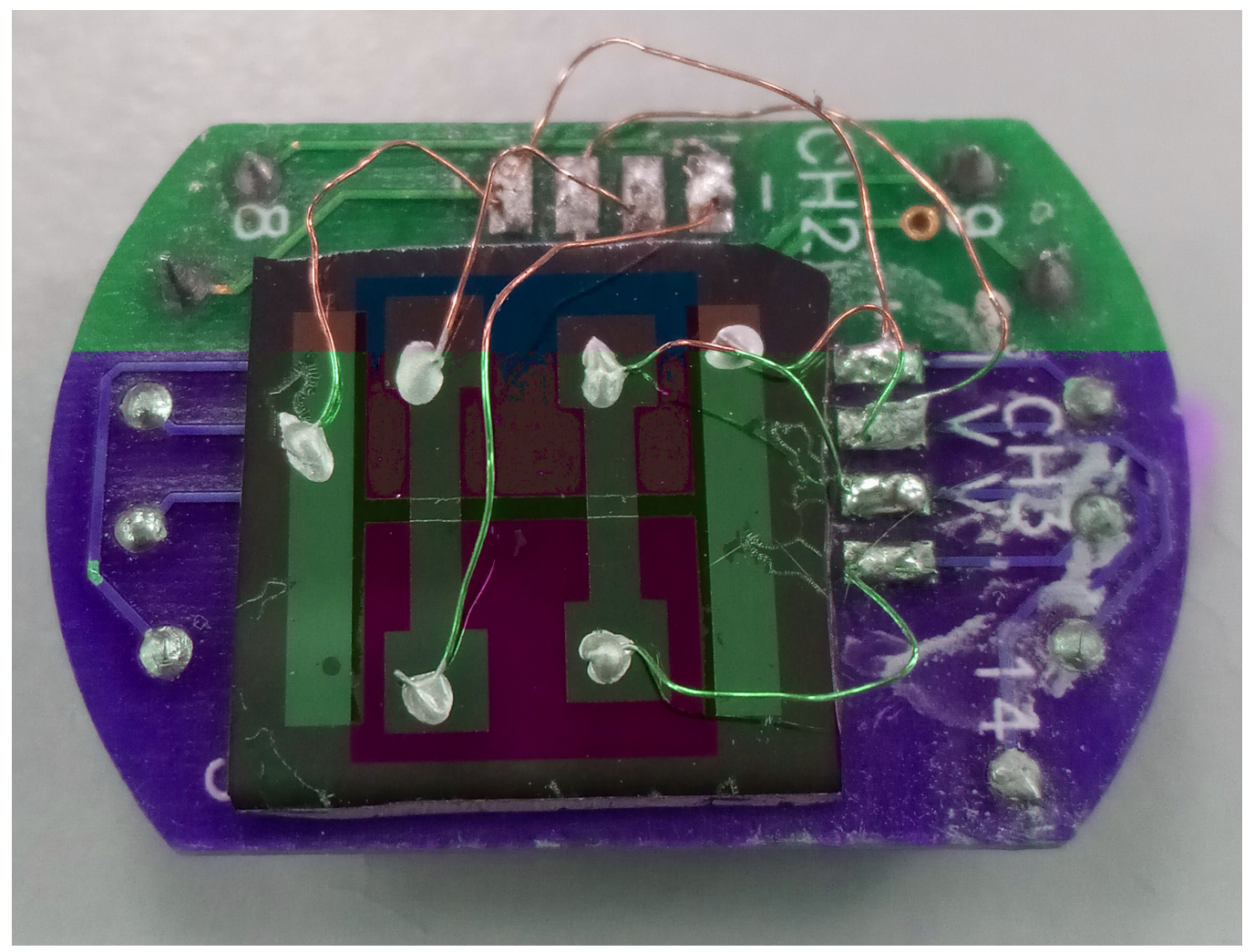

Figure 3.15: A sample mounted for the PPMS using GE-varnish, silver paint and wire. This sample has two devices mounted for simultaneous measurementes in the PPMS.

\section{QuantumDesign Magnetic Property Measurement System}

The QuantumDesign Magnetic Property Measurement System (MPMS) measures the magnetic moment. For the rare earth nitrides, it is primarily used to measure the magnetic moment as a function of temperature or applied field. We measure zero-field cooled, and field cooled temperature dependent magnetic moment, to among other things extract the Curie temperature. We also measure magnetic hysteresis loops, from which we can find the saturation magnetisation and the coercive field. 


\subsubsection{Structural characterisation}

Samples were measured in the X-ray diffractometer and the scanning electron microscope to determine their structural properties.

Using X-ray Diffraction (XRD), we can measure a number of crystallographic properties of a sample. The measurements are done using a PANalytical X'Pert PRO. By doing $2 \theta-\omega$ scans, we can find the relative abundance of different crystallographic orientations in the sample, by studying at which incident angles of the X-rays the sample displays constructive interference. Using the peak width, it is possible to extract the average size of crystallites in the sample using the Sherrer formula.

The Scanning Electron Microscope (SEM) used is a JEOL 6500F. It has multiple measurement options, but for this thesis, it was mainly used to measure cross-sectional images of thin semiconductor films. Two main measurement modes were used: secondary electron imaging (SEI) and backscattered electron imaging (BSI). In SEI the measured electrons are emitted from close to the sample surface which gives a high-resolution image of small surface features. In BSI the measured electrons originate from a deeper region of the sample, as the measured electrons are the elastically backscattered incoming electrons. In BSI the signal intensity is related to the atomic number of the atoms in the imaged sample, leading to some elemental sensitivity.

The Energy-dispersive X-ray Spectroscopy (EDS) option of the JEOL 6500F SEM was used to probe the elemental composition of samples further. EDS creates an image similar to the BSI image, but with true elemental resolution. The physical resolution is limited, but good enough to distinguish $100 \mathrm{~nm}$ layers in cross-sectional orientation. The EDS maps take hours to complete for a single image, while an SEI or BSI image take minutes. 


\section{Chapter 4}

\section{Rare earth reactions with $\mathbf{N}_{2}$}

This chapter presents a study of the formation reaction of RENs and how we can use our knowledge of the formation reaction to understand the growth of the RENs using $\mathrm{RE}+\mathrm{N}_{2}$. We show an alternative way of making and doping RENs by exposure to $\mathrm{N}_{2}$ post-deposition. We show that the nitrogen in the top $2 \mathrm{~nm}$ of $\mathrm{Sm}, \mathrm{Gd}, \mathrm{Dy}$, and $\mathrm{SmN}$ films is mobile. As precise control over the REN composition is required, particularly for future device applications, this chapter details some $\mathrm{N}_{2}$ related considerations for consistent film growth. To the author's knowledge, this work presents the first real-time experimental measurements of the nitriding process.

The cracking of the nitrogen molecule by RE ions during the formation of the RENs has been utilised to grow RENs for many years, but the reaction itself has never been studied in detail $[1,2,41]$. The reaction can happen at pressures as low as $10^{-5}$ mbar and ambient substrate temperatures [1]. It is uncommon for elemental metals to react so readily with $\mathrm{N}_{2}$ due to the high binding energy of $\mathrm{N}_{2}$ [42-44]. The rarity of the nitrogen cracking reactions makes the $\mathrm{RE}+\mathrm{N}_{2}$ reaction even more interesting to study.

We show that by depositing a pure, clean RE thin film and exposing it to $\mathrm{N}_{2}$ we turn the RE into REN. We measure the in-situ electrical conductance during the nitrogen exposure and study reaction rates, nitriding 
depth profiles, and study the diffusion into the RE layers.

We also show that it is possible to remove and reintroduce nitrogen semi-reversibly in thin REN films which have been heavily doped during growth and contain a high concentration of nitrogen vacancies. The relative conductance change in the RE and REN films are similar when exposed to the same dose of $\mathrm{N}_{2}$.

The pressures at which the pure RE reacts with and incorporates nitrogen and the pressure at which the REN desorbs nitrogen from the film gives us an estimate of the range of pressures we can use to manipulate the nitrogen content in a REN.

\subsection{Background of nitriding}

The word nitriding is used in literature to describe many processes involving nitrogen. In this thesis, we use nitriding and nitridation to mean the process of forming a nitride from an elemental film using $\mathrm{N}_{2}$.

Most elements require nitrogen to be ionised or at least atomic to be able to react and form a nitride. This is a result of the large energy required to break the nitrogen bond, which is not readily available at most material surfaces. Molecular nitrogen is known as an inert gas thanks to its small bond length $(110 \mathrm{pm})$ and high bond dissociation energy $(9.79 \mathrm{eV} /$ bond $)$ [45]. It has the second strongest diatomic chemical bond, not far behind carbon monoxide which has a bond-dissociation energy of $11.1 \mathrm{eV} /$ bond [45].

Nitriding is well understood in a variety of metals, with a particular focus on iron compounds [46-49]. Steel has been receiving much attention thanks to its importance in all manufacturing, as nitriding is used to harden the surface and prevent oxidation [49-52].

There are multiple ways of nitriding a surface, for example; plasma ion implantation, beam ion implantation, ion nitriding, and gas nitriding. Plasma ion implantation and beam ion implantation are used for deep 
and high concentration (20\% to $30 \%$ ) nitriding of a surface, often to more than $1 \mu \mathrm{m}$ deep [53]. Ion nitriding and gas nitriding only nitride to less than $1 \mu \mathrm{m}$ and achieve lower nitrogen concentrations [53]. Gas nitriding is the process that most closely resembles the RE $+\mathrm{N}_{2} \rightarrow$ REN growth. Gas nitriding is often done with ammonia and heated surfaces but can, in theory, be done with any unactivated gas, such as with molecular nitrogen, if the surface is catalytically active.

While it is a rather rare process, gas nitriding can be done under a variety of conditions such as different temperatures, different surface preparations, and different gas mixtures. In gas nitriding, the nitriding material is often a $>1 \mu \mathrm{m}$ thick film. However, gas nitriding can also be performed on powders or powders in a ball mill which can achieve higher nitriding percentages ( $>40 \%$ ) than thin film nitriding [54-56].

An important concept in nitriding in the nitrogen equilibrium pressure, which is the pressure at which the rates of nitrogen atoms desorbing from the film and adsorbing into the film are the same. Thus, at the equilibrium pressure the film is in equilibrium with the surrounding atmosphere. The nitrogen equilibrium pressure depends on many things such as nitride composition, stoichiometry, and temperature [57].

\subsection{Rare earth changes during nitriding}

In this section, we will start by discussing what we can learn from monitoring the conductance of different RE films pre-nitriding and how the structure of the RE film can influence the nitriding reaction. Subsequently, we will move on to results from in-situ nitriding the grown RE thin films. Finally, we will discuss how REN films can be nitrided and de-nitrided in-situ post-deposition. 


\subsubsection{Experimental details}

All experiments were performed using the in-situ conductance setup described in section 3.6.1. A diagram of the experiments performed is shown in fig. 4.1. The data collected during the experiments was: the resistance of the nitriding RE film, the pressure in the vacuum chamber, time, thickness, and growth rate.

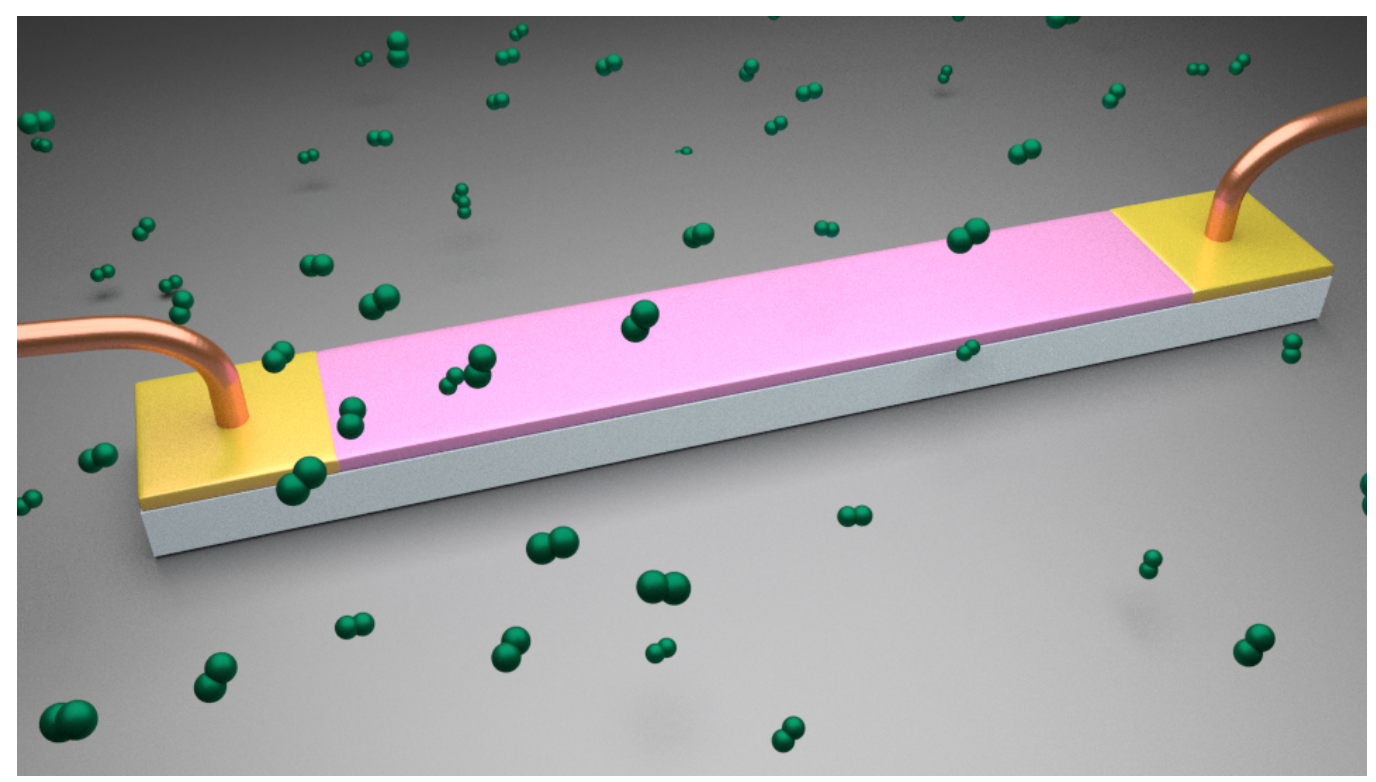

Figure 4.1: In-situ electrical setup described in section 3.6.1 with a RE film (pink) deposited between the gold contacts and $\mathrm{N}_{2}$ molecules (green) in the chamber. Not to scale.

A thin RE film was deposited between two pre-deposited gold contacts where the resistance was measured in a two-point configuration. The substrates were single side polished $\mathrm{Al}_{2} \mathrm{O}_{3}$ wafer cut to $10 \mathrm{~mm} \times 10 \mathrm{~mm}$. The gold contacts were patterned using a shadow mask and grown $20 \mathrm{~nm}$ thick using thermal evaporation.

The substrate with pre-deposited contacts was loaded into the UHV system where electrical leads were attached to the gold contacts using indium, and a $2 \mathrm{~mm} \times 10 \mathrm{~mm}$ strip was masked off for the growth. The UHV 
chamber and material sources were outgassed and baked for one week prior to each experiment.

The RE films were deposited from a resistive boat or an electron gun. The background pressure during RE deposition was kept below $2 \cdot 10^{-7} \mathrm{mbar}$ to ensure a clean RE film. The RE films were grown to a thickness of $15 \mathrm{~nm}$ to $20 \mathrm{~nm}$ as indicated by a crystal balance at which point a shutter was placed in front of the sample. The thicknesses indicated by the crystal balance were scaled using the sample dimensions and resistivity of the RE to give the thicknesses reported in this chapter.

Before starting the $\mathrm{N}_{2}$ exposure, the RE source was turned off to decrease the background pressure and to stop further RE growth. $\mathrm{N}_{2}$ was introduced at pressures between $1 \cdot 10^{-4} \mathrm{mbar}$ and $3 \cdot 10^{-4} \mathrm{mbar}$ through a MKS mass flow controller set from $5.2 \mathrm{sccm}$ to $10 \mathrm{sccm}$.

\subsubsection{Film growth}

The nitriding of the rare earths occurs without externally added energy which means the RE surface acts as a catalyst. Catalytic reactions, in general, depend heavily on the surface morphology [43]. We, therefore, start this section by studying the growth of Sm thin films to understand how the surface of the films may look. We study the film growth using the conductance change with thickness as this shines a light on some film properties in a non-invasive measurement which does not change the surface for the subsequent nitriding experiment.

The conductance of four, identically prepared within experimental accuracy, pure $\mathrm{Sm}$ films as a function of the thickness during growth is shown in fig. 4.2. The conductance of all films starts at zero, and after $1.8 \mathrm{~nm}$ to $4 \mathrm{~nm}$ of Sm has been deposited the conductance becomes measurable. Once the conductance becomes finite, there are only minor differences in the shape of the conductance onset between the films before levelling out to a linear conductance increase with thickness. 


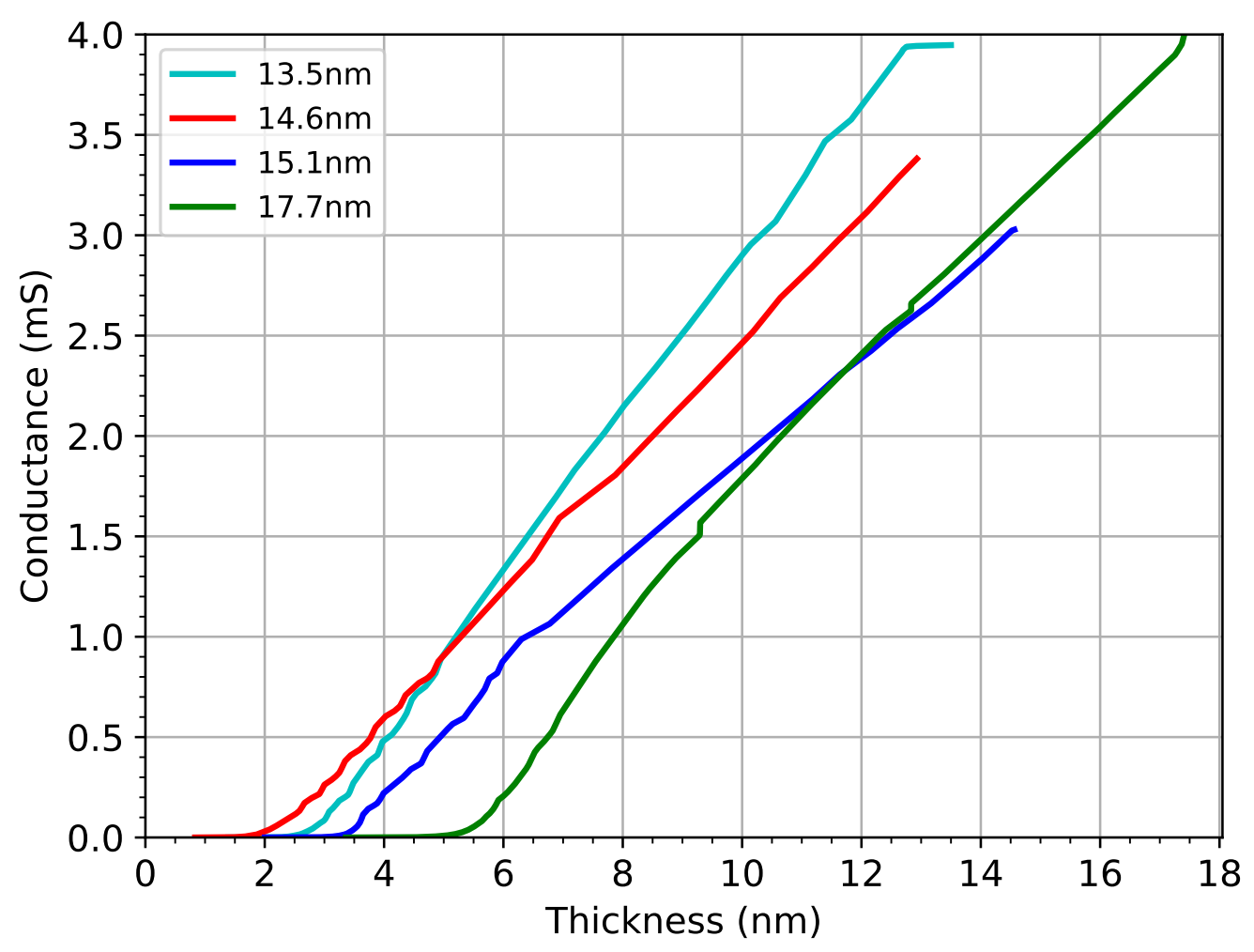

Figure 4.2: Conductance vs thickness of four, nominally identical, Sm samples being grown. Thickness calculated by assuming $\rho_{S m}=94 \mu \Omega \mathrm{cm}$. Legend shows final film thickness.

We attribute the increase in conductance between $1.8 \mathrm{~nm}$ and $4 \mathrm{~nm}$ in fig. 4.2 to islands touching and forming a continuous film, as described in section 2.1. The Sm forms islands on the substrate which reach the percolation threshold to form a continuous current path. We assume that the islands grow as fast in-plane as out-of-plane before touching (hemispherical growth) which means that the islands would be $3.6 \mathrm{~nm}$ to $8 \mathrm{~nm}$ in diameter before the individual islands touch and the percolation threshold is reached, as per fig. 2.1. The percolation threshold is reached at a small island size which we interpret as a high density of initial islands, which in turn implies a large sticking coefficient of the Sm both to the substrate and 
to the already deposited Sm.

The island growth is not surprising as metals often grow in an island growth mode, also known as Volmer-Weber growth mode [10,58], which is discussed in more detail in section 2.1.

A small island size at the percolation threshold suggests that there will be many grain boundaries and that the grain size in-plane will be small. Small grain sizes and long grain boundaries are known to increase reaction rates with gases as there are more exposed reaction sites [59,60]. Polycrystalline RE films, as presented in this chapter, are likely to be nitrided more easily than an equally thick epitaxial RE film.

Once the islands have formed a conduction path, the conductance rises approximately linearly. Assuming the Sm conductivity does not change with thickness the linear conductance increase can be attributed to a linear increase in thickness - implying a smooth metal growth once the islands have formed a continuous current path. Assuming the film becomes homogeneous and is ohmic the linear conductance increase with thickness is expected.

The final thickness of the Sm films is calculated using eq. (2.6) with the literature value for the resistivity of $\operatorname{Sm}\left(\rho_{S m}=94 \mu \Omega \mathrm{cm}\right)$, and measured values of conductance $(G)$, film length $(\ell)$, and film width $(w)$. The calculated thickness for the five samples is comparable to the thickness as measured by the crystal balance.

A relevant comparison to make before discussing the reaction with $\mathrm{N}_{2}$ is comparing $\rho_{S m}=94 \mu \Omega \mathrm{cm}$ to typical conductivities of $\mathrm{SmN}$ and other RENs, such as in section 2.3. Typical REN conductivities are $>100 \mathrm{~m} \Omega \mathrm{cm}$, at least three orders of magnitude larger than the Sm films presented in fig. 4.2 [2,39]. 


\subsubsection{Structure changes from nitriding Gd}

Before studying the real-time nitriding of REs, we demonstrate that nitriding does turn the RE into a REN. Figure 4.3 shows the XRD spectra of a $\mathrm{Gd}$ film which has not been nitrided as well as a Gd film which has been nitrided as described above.

The pure Gd film has three different Gd crystallographic orientations in the XRD spectrum as well as a GaN peak from the capping layer. When studying the XRD spectrum of a nitrided Gd film, the Gd peaks are no longer visible but a clear GdN peak has appeared.

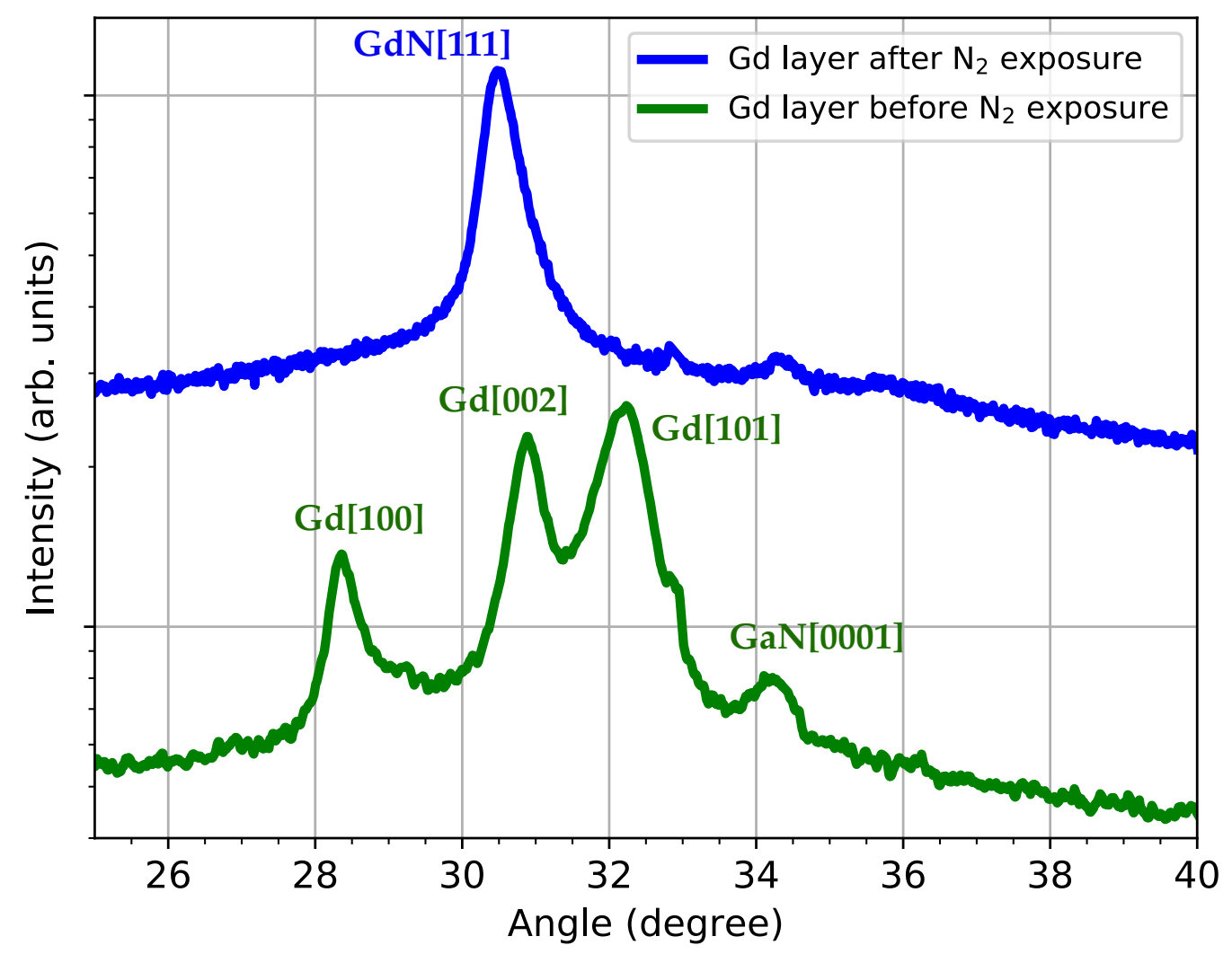

Figure 4.3: XRD 2 $\theta-\omega$ spectra of a $25 \mathrm{~nm}$ pure Gd film before exposure to $\mathrm{N}_{2}$ and a $25 \mathrm{~nm} \mathrm{Gd}$ film after exposure to $\mathrm{N}_{2}$ showing the transformation from $\mathrm{Gd}$ to $\mathrm{GdN}$ upon nitriding. 
The extinction of the Gd peaks in the nitrided Gd suggests that the nitriding affects the $\mathrm{Gd}$ throughout the entire $25 \mathrm{~nm}$ film. While the XRD tells us that the nitriding of a RE produces a REN, it does not tell us how the nitriding affects the RE film in real time which we will discuss below.

\subsubsection{Conductance changes during nitriding of Sm}

To study how the RE- $\mathrm{N}_{2}$ reaction happens we will make use of the fact that the RE metal has at least three orders of magnitude higher electrical conductivity than the equivalent REN. We measure the conductivity of the RE film as we expose it to $\mathrm{N}_{2}$ and infer how much of the film has turned into a REN.

We describe the thin RE film as a two-dimensional network of parallel resistors from the surface to deep inside the film as seen in fig. 4.4. We measure the total conductance of a thin film as the sum of the conductance of all of the parallel resistors whether they are RE or REN resistors, as described in eq. (4.1), where $x$ is the fraction of the film that has become nitrided, and $G$ is the conductance (total, RE, or REN). This model assumes a step function in concentration difference between nitrided and non-nitrided layers. Equation (4.1) can be expanded into eq. (4.2) where $w$ is the width of the film, $\ell$ is the length of the film, $t$ is the total thickness of the film, $\sigma$ is the conductivity (RE or REN), and $\tau$ representing time. As the nitriding progresses the nitrided fraction, $x$, will increase.

$$
\begin{aligned}
& G_{\text {total }}=\Sigma\left[(1-x) \cdot G_{R E}+x \cdot G_{R E N}\right] \\
& G_{\text {total }}(\tau)=\frac{w t}{\ell}\left[(1-x(\tau)) \cdot \sigma_{R E}+x(\tau) \cdot \sigma_{R E N}\right]
\end{aligned}
$$

As the semiconducting REN resistors will have negligible conductance compared to the metallic RE resistors, we assume they do not contribute to the total film conductance, leading us to equation 4.3.

$$
G_{\text {total }}(\tau)=\frac{w t}{\ell}\left[(1-x(\tau)) \cdot \sigma_{R E}\right]
$$




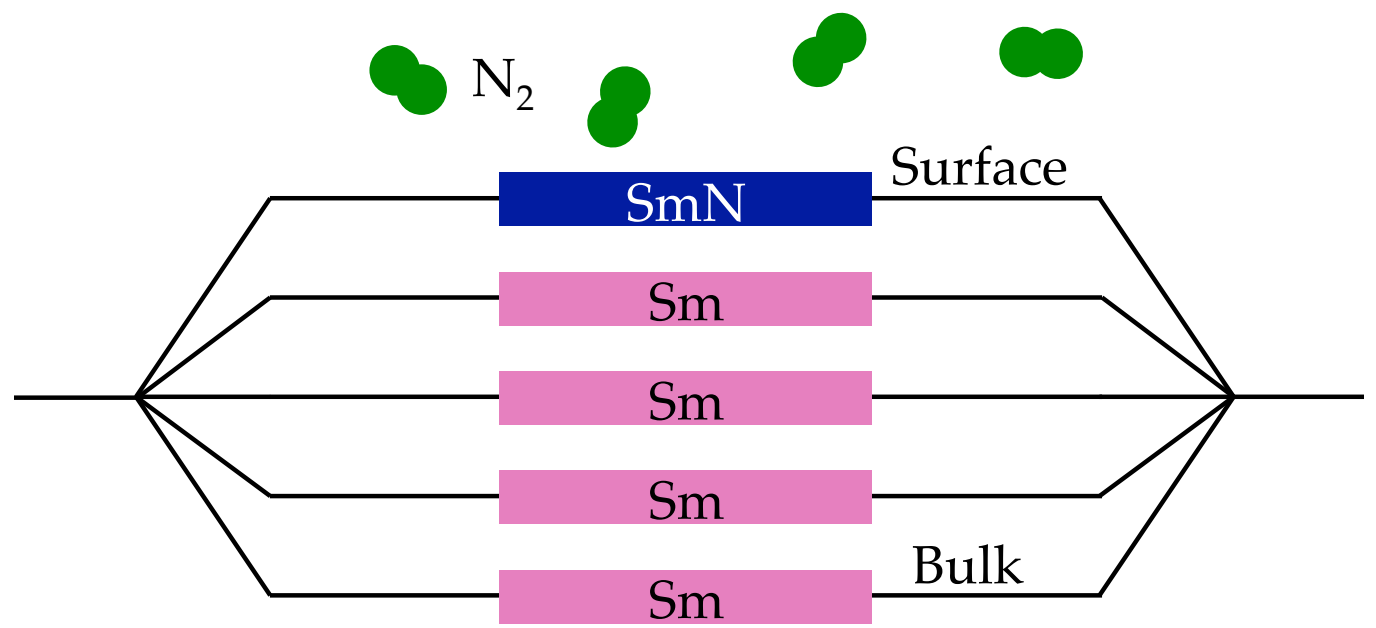

Figure 4.4: A diagram of the parallel resistor model for the nitriding of Sm propagating from the surface.

The fraction of the film that has become nitrided at any time, $x(t)$, can be found as

$$
x(\tau)=\frac{G(0)-G(\tau)}{G(0)}
$$

showing that the fraction on the film that is nitrided is the relative conductance change in the film.

This model will aid us in interpreting the results of the nitriding; however, it is too simple to describe the subtler features of the process as we assume we only have fully nitrided REN or completely non-nitrided RE layers. We will briefly discuss more realistic models below.

An overview of a complete nitriding in-situ electrical experiment is shown in fig. 4.5. The figure shows the film conductance and chamber pressure as a function of time. In panel $\mathrm{A}$, the initial conductance rises due to the growth of the RE thin film, discussed in detail in section 4.2.2. When the film has reached the desired thickness, in this case after about $500 \mathrm{~s}$ and $15 \mathrm{~nm}$, the RE metal growth is stopped.

In panel $B$, the chamber pressure drops as the RE is cooled down from evaporation temperature; meanwhile, the conductance is stable. The slight 


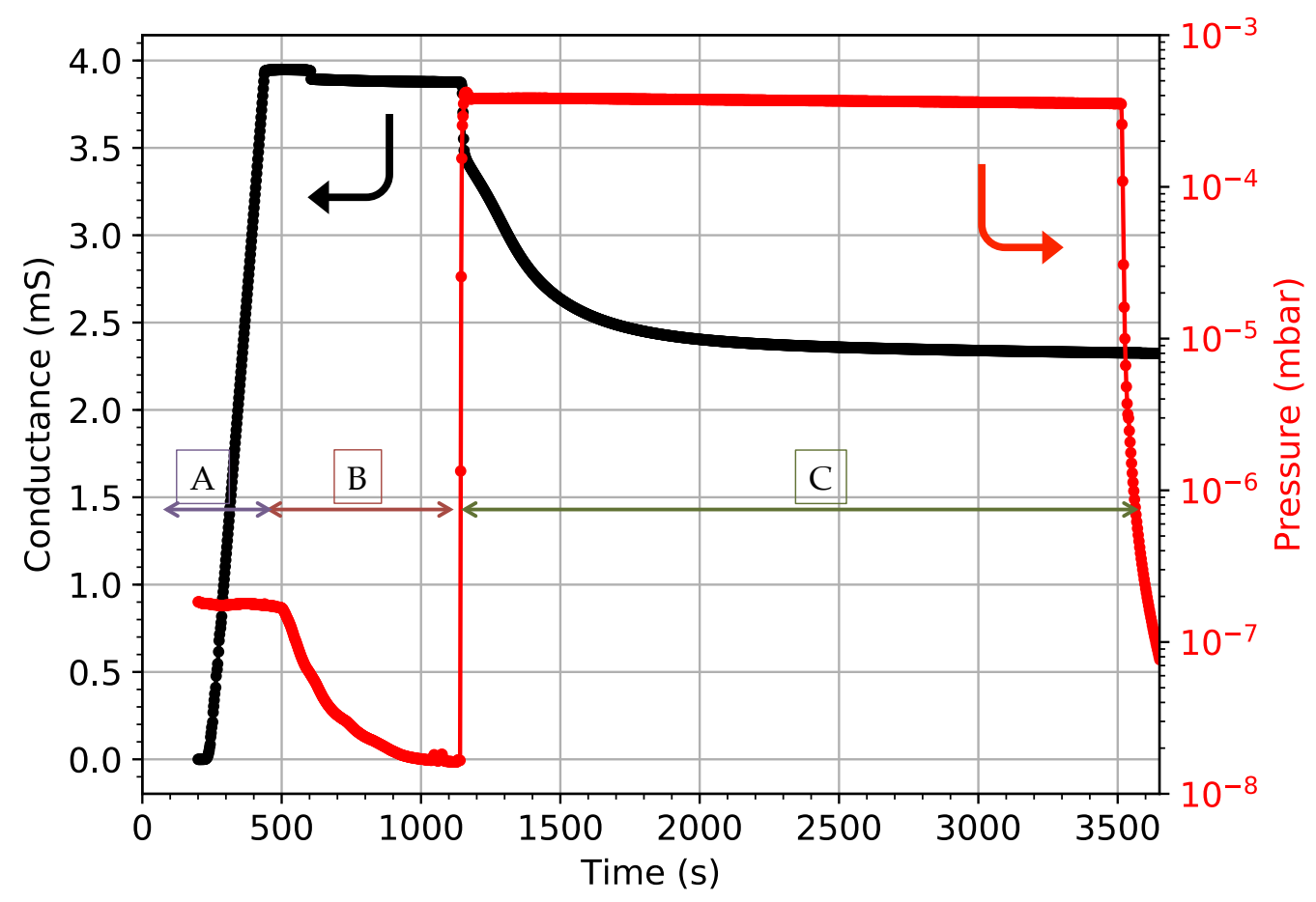

Figure 4.5: Nitriding of a $15 \mathrm{~nm}$ thin Sm film. A - Sm film growth. B cooling down $\mathrm{Sm}$, no reaction. $\mathrm{C}-\mathrm{N}_{2}$ introduced in chamber to nitride the Sm.

step is an experimental artefact. Once the RE source is at room temperature and the background pressure is lower, around 1200 seconds, the nitriding phase can begin.

In panel $\mathrm{C}, \mathrm{N}_{2}$ is introduced in the chamber at $3.8 \cdot 10^{-4}$ mbar. When we introduce $\mathrm{N}_{2}$, the conductance immediately drops by $10 \%$, even before the $\mathrm{N}_{2}$ has reached its set steady state pressure. An expanded view of the transition from panel B to $C$ can be seen in fig. 4.6.

The magnification of the moment of $\mathrm{N}_{2}$ introduction into the chamber shows the initial rapid conductance drop in fig. 4.6. Over the approximately $15 \mathrm{~s}$ it takes for the UHV chamber to fill and stabilise to the desired $\mathrm{N}_{2}$ pressure (red data points) the relative conductance of the film drops by $\sim 10 \%$ (black data points). Using eq. (4.4) we find the initial nitrided thick- 
ness in fig. 4.6 as $1.5 \mathrm{~nm}$ that is fully nitrided during initial gas pressure stabilisation.

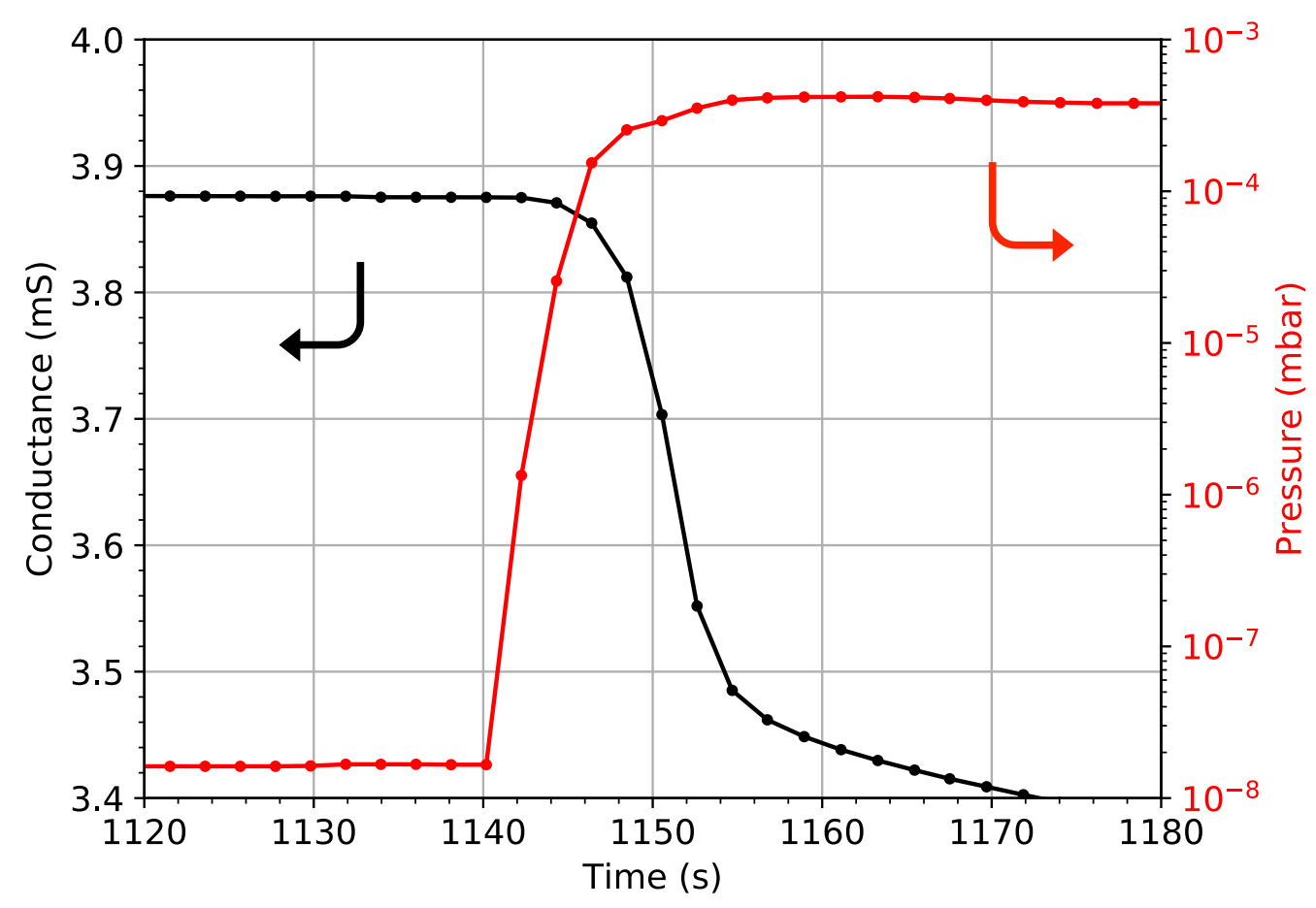

Figure 4.6: Magnification of the initial in-situ conductance drop of a $15 \mathrm{~nm}$ $\mathrm{Sm}$ as $\mathrm{N}_{2}$ is introduced.

The conductance starts dropping once the pressure in the chamber is between $1.4 \cdot 10^{-5} \mathrm{mbar}$ and $3.5 \cdot 10^{-5} \mathrm{mbar}$. If we identify this pressure range in the pressure-conductivity graph of GdN (fig. 2.8) this is at the lower pressure ranges of where $\mathrm{Gd}$ reacts with $\mathrm{N}_{2}$ to form $\mathrm{GdN}$ during continuous growth. We will see in the next chapter that the same is true for $\mathrm{SmN}$ (see fig. 5.1). The minimum $\mathrm{N}_{2}$ pressure during continuous $\mathrm{GdN}$ or $\mathrm{SmN}$ growth is the same as the $\mathrm{N}_{2}$ pressure at which Sm start to turn into $\mathrm{SmN}$. This suggests we have found a maximum threshold for the nitrogen equilibrium pressure in $\mathrm{SmN}$, i.e. $\mathrm{N}_{2}$ partial pressures above $1.4 \cdot 10^{-5}$ mbar will introduce nitrogen in the lattice.

The rapid initial conductance drop in fig. 4.6 lasts $15 \mathrm{~s}$ to $20 \mathrm{~s}$ and results 
in the top $1.5 \mathrm{~nm}$ to $2 \mathrm{~nm}$ becoming nitrided, according to eq. (4.4). The nitrided thickness during this time divided by the time for this conductance drop yields a nitriding rate of $\sim 7 \mathrm{~nm} / \min (\sim 400 \mathrm{~nm} / \mathrm{h})$. There appears to be no correlation, within the ranges tested, with pressure $\left(1 \cdot 10^{-4} \mathrm{mbar}\right.$ to $\left.4 \cdot 10^{-4} \mathrm{mbar}\right), \mathrm{N}_{2}$ flow $(5.2 \mathrm{sccm}$ to $10 \mathrm{sccm})$, or Sm initial thickness $(13 \mathrm{~nm}$ to $19 \mathrm{~nm}$ ) (data not shown explicitly but can be deduced from fig. 4.7).

The initial rapid conductance drop as seen in fig. 4.6 can be seen as nitriding of a fixed thin Sm layer. Assuming the rapid initial nitriding is representative of the $\mathrm{Sm}+\mathrm{N}_{2}$ reaction we can find an upper bound for the $\mathrm{Sm}+\mathrm{N}_{2}$ reaction rate as we have essentially measured the maximum $\mathrm{N}_{2}$ cracking and incorporation rate into $\mathrm{Sm}$. When growing $\mathrm{SmN}$ using $\mathrm{Sm}+\mathrm{N}_{2}$, the Sm deposition rate needs to be below $7 \mathrm{~nm} / \mathrm{min}$ to avoid forming a film with pure $\mathrm{Sm}$ layers. For $\mathrm{Sm}$ deposition rates above $7 \mathrm{~nm} / \mathrm{min}$ a new Sm layer will be deposited before the previous one has had time to completely form a nitride which will give an $\mathrm{Sm} / \mathrm{SmN}$ mixed film. An $\mathrm{Sm} / \mathrm{SmN}$ mixed film can be difficult to spot, particularly in multilayer structures where measurements are complex to interpret. Based on the nitriding rate of thin Sm layers we suggest that to ensure pure $\mathrm{SmN}$ in films grown at room temperature, the Sm deposition rate should be kept below $7 \mathrm{~nm} /$ min during $\mathrm{Sm}+\mathrm{N}_{2}$ growth in $1 \cdot 10^{-4}$ mbar to $4 \cdot 10^{-4}$ mbar of $\mathrm{N}_{2}$.

The part of the experiment that we will study more closely is the reaction between the RE surface and $\mathrm{N}_{2}$ - panel $\mathrm{C}$ in fig. 4.5. Based on the experimental results we expect the nitriding to be controlled by the total amount of $\mathrm{N}_{2}$ that reach the RE surface, rather than the $\mathrm{N}_{2}$ pressure or time themselves. Hence, we investigate the nitriding for the total amount of $\mathrm{N}_{2}$ which has been introduced into the UHV chamber.

We plot the conductance change (eq. (4.4)) of four Sm films as a function of $\mathrm{N}_{2}$ dose (the integral of chamber pressure over time) introduced in the chamber in fig. 4.7. By plotting the conductance change as a function of the total introduced dose of $\mathrm{N}_{2}$, we can compare different experiments more easily as well as draw conclusions of the timescales involved. For 
the dose we use the unit Langmuir $\left(1 \mathrm{~L}=1 \cdot 10^{-6}\right.$ Torr s). The dose on the $\mathrm{x}$-axis is the dose of $\mathrm{N}_{2}$ introduced in the chamber, not the dose that has reacted with the sample, although they may be proportional [61].

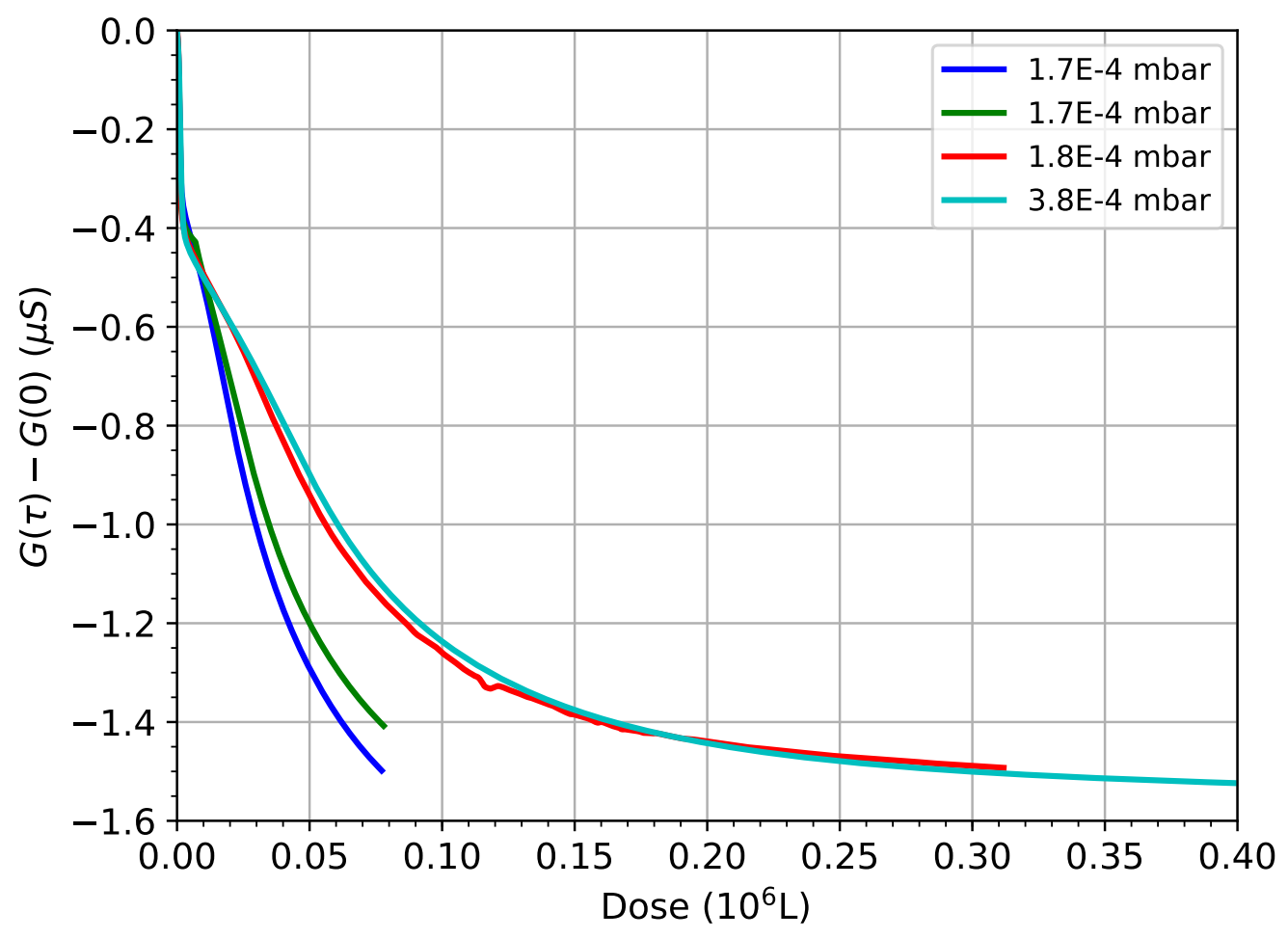

Figure 4.7: Conductance change in $4 \mathrm{Sm}$ films for increasing nitrogen exposure. Legend shows nitrogen pressure during nitriding. Colours correspond to same samples as in fig. 4.2.

Throughout fig. 4.7 the conductance decreases monotonically with increasing $\mathrm{N}_{2}$ dose. A spread in behaviour for similar $\mathrm{N}_{2}$ chamber pressures suggest there are uncontrolled variables in the experiment. As per fig. 4.7 we have identified three phases of the nitriding: rapid initial conductance drop, conductance pause, and final conductance plateau which we will discuss in turn.

The initial rapid conductance drop in fig. 4.7 corresponds to $1.5 \mathrm{~nm}$ to $2 \mathrm{~nm}$ of the RE being nitrided for the four films, as calculated using eq. (4.4) 
using the model described above. The surface morphology is known to determine how much of the film, in general, is easily accessible to react with a nitrogen molecule without requiring diffusion through grain boundaries or already nitrided layers [50]. The thickness calculated from the initial conductance drop correlates with the initial island radius ( $1.8 \mathrm{~nm}$ to $4 \mathrm{~nm}$ ), and if this initial island size is retained as the grain size throughout the films, this suggests the top layer of the Sm film has a similar roughness. This, in turn, suggests that the RE film structure does affect the nitriding process.

After the initial rapid conductance drop in fig. 4.7, there is a slight pause $\left(0.01 \cdot 10^{6} \mathrm{~L}<\right.$ Dose $\left.<0.05 \cdot 10^{6} \mathrm{ML}\right)$. It is common in other materials to see the nitrogen diffusivity drop with an increasing nitrogen content in the lattice [50]. We propose that the pause comes from a change from a reaction regime to a diffusion-limited regime due to the surface layers becoming $\mathrm{SmN}_{x}, x \leq 1$. The diffusion-limited regime is likely either dominated by atomic nitrogen diffusing from the $\mathrm{SmN}$ to the Sm layers below or molecular nitrogen diffusing through the $\mathrm{SmN}$ to react with the Sm below. An initial attempt to model the nitriding of Sm shows similarities to the oxidation of silicon, as described by the Deal-Groves model (fit not shown) [62]. The Deal-Groves model assumes a three-step process: Adsorption of $\mathrm{O}_{2}$ on the top layer, transport of $\mathrm{O}_{2}$ through the surface oxide layer towards the pure silicon below, and finally reaction between the $\mathrm{O}_{2}$ and silicon to form a new oxide layer [62].

If nitrogen diffuses through the $\mathrm{SmN}$ as $\mathrm{N}$-ions this suggests that there always need to be some free $\mathrm{Sm}$ sites on the surface which can catalyse the $\mathrm{N}_{2}$ cracking. This means that as the surface $\mathrm{N}$-coverage increases there are fewer catalytic sites, which will lower the number of $\mathrm{N}$-ions diffusing into the film. Once all the surface Sm atoms, or all easily accessible empty $\mathrm{Sm}$ sites, have been bonded with an $\mathrm{N}$-ion the nitriding will stop. If nitrogen diffuses through the $\mathrm{SmN}$ as $\mathrm{N}_{2}$ this suggests that the number of catalytically active $\mathrm{Sm}$ sites on the surface is less important. The diffusion 
of the $\mathrm{N}_{2}$ through the $\mathrm{SmN}$ layer becomes lower as the $\mathrm{SmN}$ layer becomes thicker. With the current setup and measurements, it is not possible for us to distinguish these two possibilities of N-diffusion through the $\mathrm{SmN}$.

After the pause (Dose $>0.05 \cdot 10^{6} \mathrm{~L}$ ) in fig. 4.7 the conductance drop forms a smooth curve which plateaus to a final conductance towards the higher doses. The samples measured in this section show a conductance plateau that levels out at a thickness corresponding to $40 \%$ to $60 \%$ which corresponds to $6 \mathrm{~nm}$ to $8 \mathrm{~nm}$ of the RE film having become REN according to the model described above. The plateau suggests the diffusion through the $\mathrm{SmN}$ layer has become rate limiting.

The exact mechanism for the nitridation is unknown, but initial computational studies by Ref [63] suggest that the $\mathrm{N}_{2}$ molecules bind more strongly close to steps on the surface. Ref [63] also find that for some RE $(\mathrm{Nd}, \mathrm{Sm}, \mathrm{Eu})$ the $\mathrm{N}_{2}$ molecule embeds itself in a RE step and dissociates to atomic $\mathrm{N}$ embedded in the top RE layer. The computed relative energy in the dissociated state compared to the initial adsorbed $\mathrm{N}_{2}$ state is on the order of multiple $\mathrm{eV}$ for all RE, suggesting that the splitting efficiency will be high.

As discussed in chapter 2 the rare earth preferentially react with oxygen and other atmospheric gas contaminants, necessitating a protective capping layer when growing rare earth nitrides to ensure that no oxidation occurs. Minimizing gas contaminants during the nitridation experiments is crucial to produce a REN film. Performing the RE growth and nitridation experiments in a background pressure of $1 \cdot 10^{-6}$ mbar results in a film with a resistance above the measurement limit of the setup ( $40 \mathrm{M} \Omega$ ). A cleaner RE surface and a purer $\mathrm{N}_{2}$ gas for nitridation is expected to give a higher nitridation efficiency.

Achieving a nitriding of $40 \%$ using gas nitriding of Sm is initially surprising as gas nitriding, in general, tends only to be able to introduce $<20 \%$ nitrogen into a thin film [53]. However, most nitriding experiments in literature are performed on thicker films $>1 \mu \mathrm{m}$, which likely explains 
why we get higher nitrogen concentrations in a $15 \mathrm{~nm}$ film where the surface layers constitute a much larger portion of the total film. Gas nitriding percentages of $40 \%$ to $60 \%$ are typically only found in ball mill nitriding, where the constant milling of the material causes new surfaces to be continuously exposed to $\mathrm{N}_{2}[55,56]$. Since our films are thinner than most thin films in literature but larger than typical ball milling crystals, it is understandable that we can nitride $\sim 40 \%$ of our thin Sm films.

To extend the scope of this study, the nitriding behaviour of two other REs was studied. Gd and Dy were chosen they are both known to break the molecular nitrogen bond [64]. Figure 4.8 shows the conductance change in three different RE films as a function of the total received dose of $\mathrm{N}_{2}$. From the data, we immediately conclude that Sm, Gd, and Dy all exhibit similar trends; initial rapid initial nitriding, pause, and a slow nitriding to a final plateau. The initial rapid nitriding has a slightly different magnitude for the different samples. The pause is quite different for the three different REs; large for $\mathrm{Gd}$, medium for $\mathrm{Sm}$, and almost non-existent for Dy. The final nitrided thickness, as calculated by eq. (4.4), in the different RE is different, largest for $\mathrm{Sm}(\sim 6 \mathrm{~nm})$, smaller for Dy $(\sim 4 \mathrm{~nm})$, and smallest for $\mathrm{Gd}(\sim 3 \mathrm{~nm})$. 


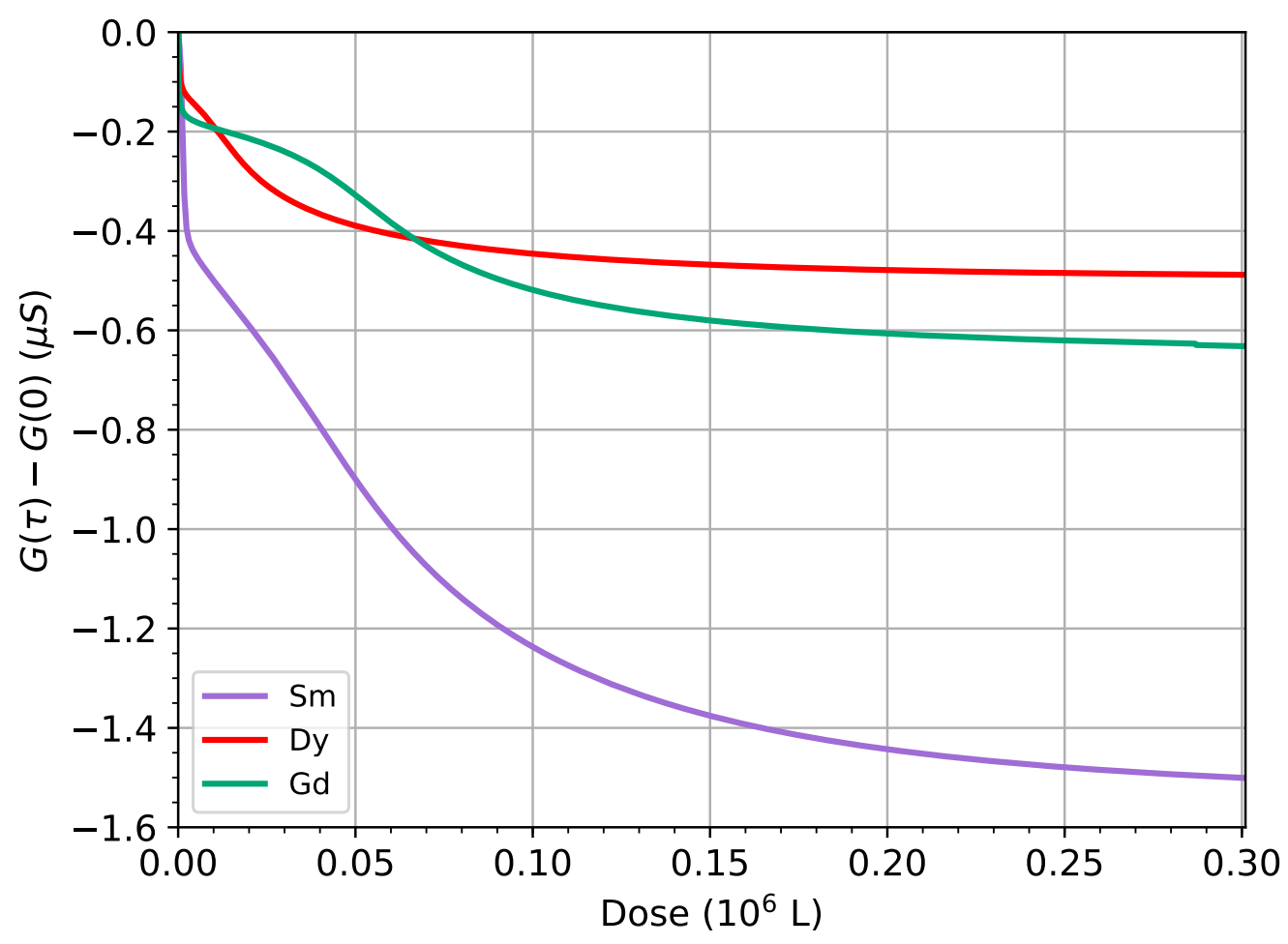

Figure 4.8: Nitriding of a three different REs.

\subsection{Extracting nitrogen from $\mathrm{SmN}$}

We have shown that $\mathrm{N}_{2}$ reacts readily with a pure $\mathrm{RE}$ surface. This section will investigate how a changing $\mathrm{N}_{2}$ pressure affects an as-grown $\mathrm{SmN}$ film, with a particular focus on what this means for electrical measurements.

We show measurements of the conductance of highly doped (nitrogenpoor) SmN thin films where the film cycles through $\mathrm{N}_{2}$ atmospheres of $1 \cdot 10^{-4}$ mbar and UHV conditions of $1 \cdot 10^{-8}$ mbar. The conductance changes with the $\mathrm{N}_{2}$ pressure in the chamber, decreasing with high $\mathrm{N}_{2}$ pressure and increasing with low $\mathrm{N}_{2}$ pressure, suggesting that the nitrogen in at least part of the film is mobile. 


\subsubsection{SmN preparation}

SmN thin films were grown using physical vapour deposition in the conductance setup described in section 3.6.1. Sm metal was evaporated from a crucible in a wire basket in an atmosphere of $\mathrm{N}_{2}$. The background pressure was $1 \cdot 10^{-8} \mathrm{mbar}$, and the $\mathrm{N}_{2}$ partial pressure was $1 \cdot 10^{-4} \mathrm{mbar}$. The purity of the $\mathrm{N}_{2}$ gas was $99.999 \%$. The resistance of the sample, the pressure in the chamber and the thickness of the sample were recorded every second.

After a typical film thickness of $40 \mathrm{~nm}$, the shutter was closed, the Sm source allowed to cool, and the $\mathrm{N}_{2}$ flow stopped. Cooling down the Sm and turning off the $\mathrm{N}_{2}$ flow allows the chamber to pump down to base pressure again after the sample has finished growing. Once the chamber returned to base pressure, the pressure experiment was started.

\subsubsection{Conductance changes in $\mathrm{SmN}$}

Figure 4.9 shows the conductance (black) of an as-grown highly doped ( $\sim 1 \%$ nitrogen vacancies, $\sigma \sim 7 \mathrm{~S} / \mathrm{cm}$ ) SmN film, and the chamber pressure (red) plotted against time in seconds. When the chamber pressure is high, the mass flow controller lets $\mathrm{N}_{2}$ into the chamber, and when the pressure is low, the $\mathrm{N}_{2}$ flow is stopped, and the chamber is pumped down to UHV conditions. Each phase is approximately $60 \mathrm{~min}$ long.

During the high $\mathrm{N}_{2}$ pressure phases in fig. 4.9 , the $\mathrm{SmN}$ conductance drops rapidly initially before continuing a steady, but slower, conductance drop. We assume that the nitrogen enters the $\mathrm{SmN}$ lattice and fills nitrogen vacancies. If we assume that the more nitrided $\mathrm{SmN}$ is much less conductive than the as-grown SmN layers, we can use the model for the nitriding of $\mathrm{Sm}$ as described above in eq. (4.4).

In the first high $\mathrm{N}_{2}$ phase, we find a thickness of $6 \mathrm{~nm}$ of more highly nitrided SmN from the rapid initial drop using eq. (4.4). The transition to a slower conductance drop suggests a drop in the diffusion of nitrogen 


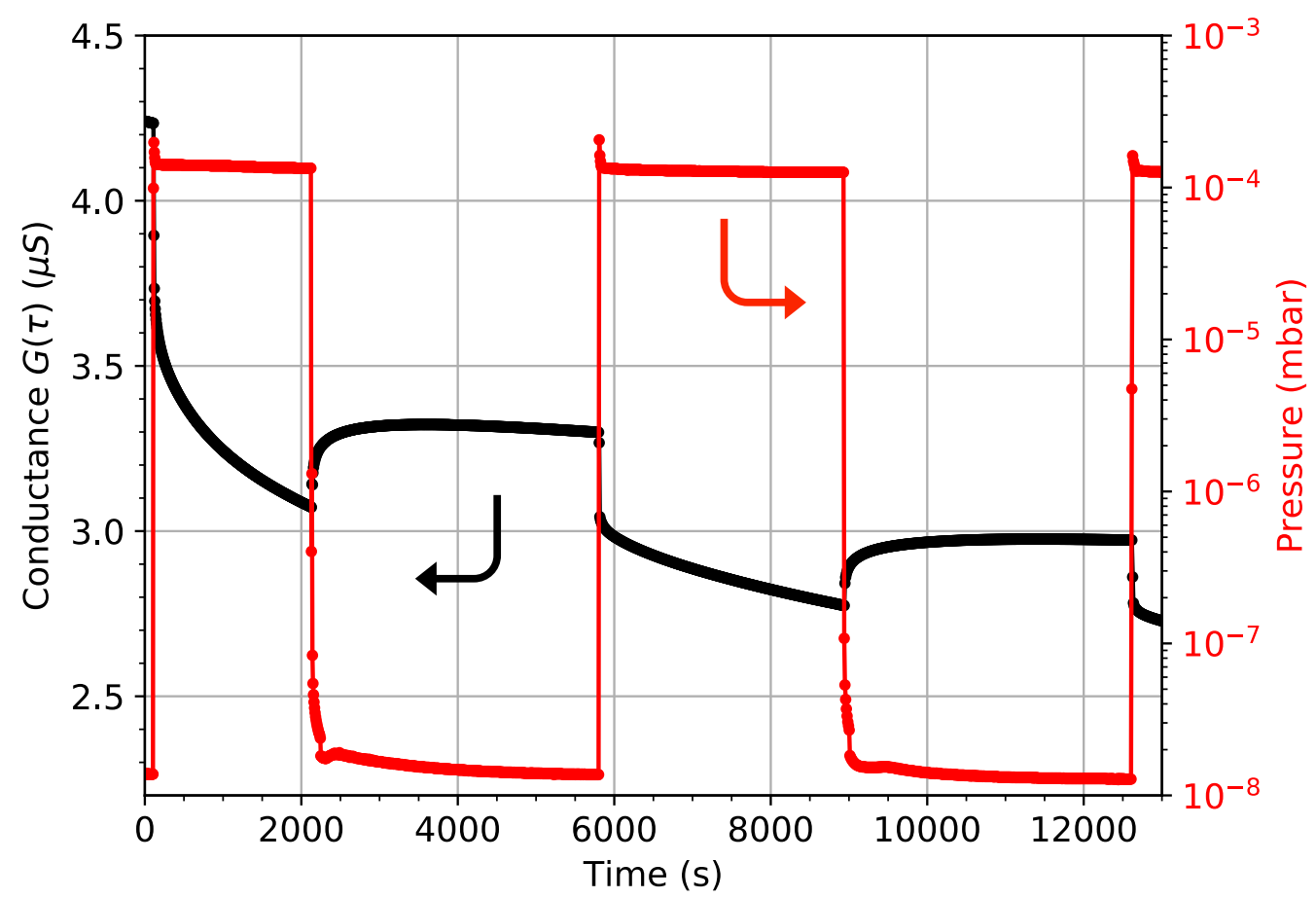

Figure 4.9: Conductance of a $40 \mathrm{~nm}$ thick $\mathrm{SmN}$ film and $\mathrm{N}_{2}$ chamber pressure against time as the $\mathrm{N}_{2}$ pressure in cycled in the UHV chamber.

through the now higher N-concentration surface layers. The final conductance change in the first $\mathrm{N}_{2}$ phase corresponds to a nitriding depth of $11 \mathrm{~nm}$. This behaviour is illustrated in the left panel in fig. 4.10 where the darker $\mathrm{SmN}$ represents $\mathrm{SmN}$ with more nitrogen in the lattice.

The conductance loss in the first $\mathrm{N}_{2}$ phase $(0 \mathrm{~s}<t<2000 \mathrm{~s}$ in fig. 4.9) in fig. 4.9 looks similar to the nitriding of Sm in fig. 4.7 but without the pause in the conductance drop. We explained the pause in the Sm nitriding as a transition from an initial reaction limited regime to a diffusion limited regime. The lack of pause in the nitriding of SmN suggests that the nitriding of the $\mathrm{SmN}$ is not limited by a surface reaction initially, but that $\mathrm{SmN}$ film has a uniform nitrogen content and the nitriding is diffusion limited from the start.

The reduction in the conductance loss over time in the first $\mathrm{N}_{2}$ phase 

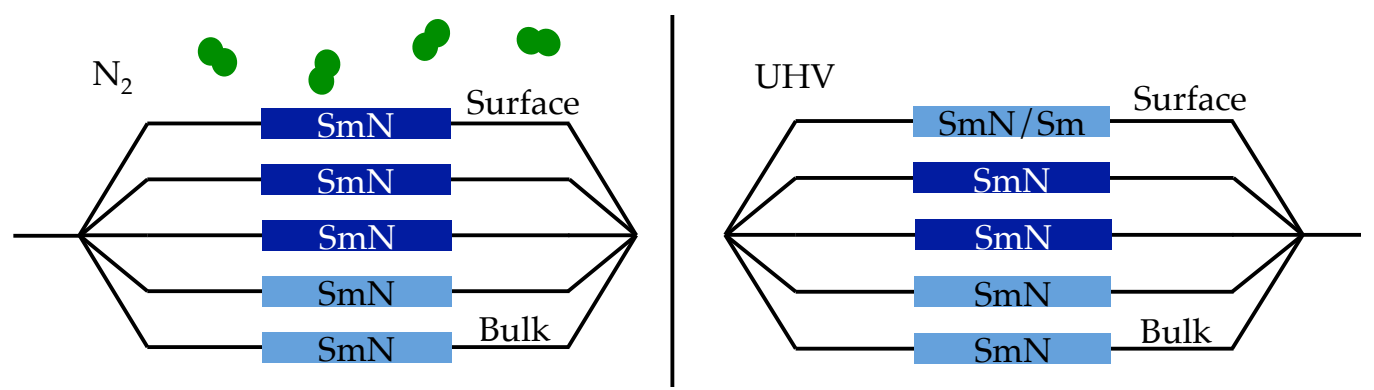

Figure 4.10: Diagram showing the nitriding and denitriding of the $\mathrm{SmN}$ film. Initially all SmN layers are identical to the bulk SmN. Darker SmN in the diagram contains more nitrogen. Only the surface layer is available for denitriding to original $\mathrm{SmN}$.

suggests that the $\mathrm{N}_{2}$ diffusion through $\mathrm{SmN}$ is dependent on the $\mathrm{N}$-concentration in the $\mathrm{SmN}$. The N-concentration dependent diffusion through $\mathrm{SmN}$ strengthens the previous argument that conductance change at high $\mathrm{N}_{2}$ dose of the $\mathrm{Sm}$ nitriding is indeed from $\mathrm{N}_{2}$ diffusing through an $\mathrm{SmN}$ layer.

When the $\mathrm{N}_{2}$ gas is pumped out of the chamber the first UHV phase $(2000 \mathrm{~s}<t<5500 \mathrm{~s}$ in fig. 4.9) in fig. 4.9, the conductance partially recovers very quickly before levelling off to a steady conductance. The levelling off implies that nitrogen cannot easily leave the SmN layers deeper in the film. This behaviour is illustrated in the right panel in fig. 4.10 where the top layers have reverted to the original nitrogen concentration SmN without affecting layers deeper in the film. The magnitude of the initial rapid conductance increase when entering a UHV phase, combined with eq. (4.4), shows that the nitrogen concentration in the top $4 \mathrm{~nm}$ has become lower or even that it is restored to the original concentration.

The conductance increase in the SmN film when it is in UHV conditions suggests that the nitrogen in the surface layers is not strongly bound. The conductance increase in UHV conditions does not occur after nitriding pure $\mathrm{Sm}$, suggesting that there is a minimum concentration of nitrogen required in the lattice before $\mathrm{N}_{2}$ can leave the film. The conductance of the 
SmN film starts increasing when the pressure drops below $5 \cdot 10^{-6}$ mbar. This suggests we have found a lower bound for the SmN nitrogen equilibrium pressure as pressures below this allow $\mathrm{N}_{2}$ to escape.

The conductance never recovers fully back to the initial SmN conductance when the chamber returns to UHV conditions. The introduction of more nitrogen into the lattice causes some irreversible conductance change. This suggests that once the nitrogen has been bound by an Sm ion, it is difficult to get it out of the lattice, particularly for SmN deeper in the film. Some of the non-reversible conductance loss could potentially be attributed to the introduced nitrogen (whether in atomic or molecular form) distorting the SmN lattice [44,65].

The conductance of the SmN film can be manipulated further by exposing the film to additional $\mathrm{N}_{2}$ and UHV phases. The conductance keeps dropping during subsequent $\mathrm{N}_{2}$ phases. The conductance recovery becomes smaller for subsequent UHV phases. This way of controlling the doping level is different to the standard way of doping during growth and opens the opportunity for some post-growth doping control of the RENs. To the author's knowledge, this method of controlling the doping level is novel and has never been reported before.

In fig. 4.9 the conductance at the end of the first high $\mathrm{N}_{2}$ phase seems to line up with the beginning of the second high $\mathrm{N}_{2}$ phase, and so on. To visualise this, we take the data from fig. 4.9 and transform the conductance into fig. 4.11 where the $x$-axis is now the total introduced dose of $\mathrm{N}_{2}$ (in $10^{6} \mathrm{~L}$ ) and the $y$-axis is the conductance loss.

In fig. 4.11 the spikes in the data indicate the UHV phase, as the conductance changes but the $\mathrm{N}_{2}$ dose does not. We can see that the conductance drop forms an almost continuous line when plotted against the dose. As more nitrogen is introduced to the $\mathrm{SmN}$ film, more nitrogen vacancies are filled, and the film conductance drops.

In fig. 4.11 we find a $1.3 \mu \mathrm{S}$ conductance loss at $0.25 \cdot 10^{6} \mathrm{~L}$. Comparing the conductance loss of the SmN to the Sm films in fig. 4.7, the conductance 


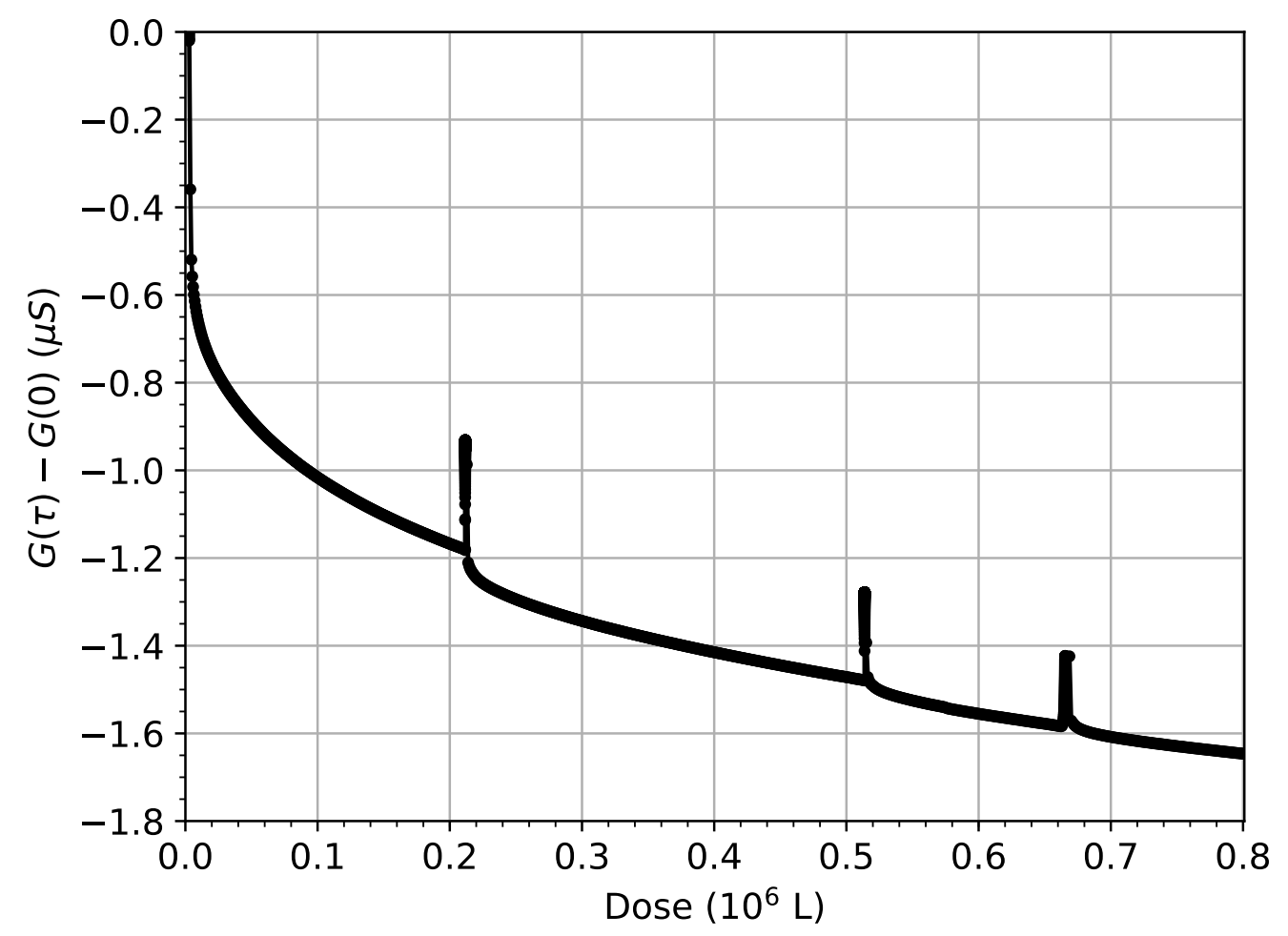

Figure 4.11: Conductance of a $40 \mathrm{~nm}$ thick $\mathrm{SmN}$ film vs chamber dose as the $\mathrm{N}_{2}$ pressure in cycled in the UHV chamber.

changes are comparable for comparable $\mathrm{N}_{2}$ doses. The nitriding depth at $0.25 \cdot 10^{6} \mathrm{~L}$ in $\mathrm{SmN}$ film is $12 \mathrm{~nm}$, more than for the Sm films in fig. 4.7 where the largest nitrided depth is $8 \mathrm{~nm}$. The larger nitriding depth in the $\mathrm{SmN}$ films suggests that the diffusion through the REN layer behaves differently when making a REN via post-growth nitriding versus making a REN with co-deposition of $\mathrm{RE}$ and $\mathrm{N}_{2}$. A different diffusion coefficient through as-deposited REN versus post-growth nitrided REN suggests that the REN crystal structure is different, which is not surprising since $\mathrm{SmN}$ is a rocksalt crystal while Sm is rhombohedral $[1,66]$. As discussed in fig. 4.3 the crystal structure of the film does change upon nitridation.

In fig. 4.11 the conductance change in the UHV phases show a smaller 
conductance increase for subsequent UHV phases. The conductance recovery in the first UHV phase corresponds to about a $\sim 4 \mathrm{~nm}$ region of the SmN with higher conductivity and the last peak in fig. 4.11 corresponding to $\sim 2 \mathrm{~nm}$. The smaller thickness recovery for a more nitrided film suggests that not only is the $\mathrm{SmN}$ film nitrided more deeply, but the nitrogen is more strongly bound in the lattice.

The rapid change in conductance at the start of each phase and the corresponding likely change in material composition near the surface of an $\mathrm{SmN}$ film has important consequences for measuring the resistance of thin REN films and multilayers of these.

\subsection{Discussion}

This chapter has demonstrated the cracking of $\mathrm{N}_{2}$ on clean RE surfaces which has not been described previously in the literature. The $\mathrm{N}_{2}$ cracking is easily performed at ambient temperature and low pressures $\left(>5 \cdot 10^{-6} \mathrm{mbar}\right)$ demonstrating that RE surfaces can be used to crack $\mathrm{N}_{2}$ in milder conditions than most other $\mathrm{N}_{2}$ cracking catalysts.

The change in crystal structure after exposing a Gd film to nitrogen demonstrates a change from $\mathrm{Gd}$ to $\mathrm{GdN}$ upon exposure to $\mathrm{N}_{2}$. The conductance measurements in this chapter are the first experiment to follow the reaction between the $\mathrm{RE}$ and $\mathrm{N}_{2}$ in real time. The conductance changes show that nitriding of a RE surface offers an alternative way of making a REN. Nitriding the RE to form a REN may be preferable to codeposition of $\mathrm{RE}$ and $\mathrm{N}_{2}$ in future manufacturing situations due to the ability to perform the RE deposition and the nitriding in different connected UHV chambers.

We find that the conductance of a REN film can be lowered post-growth in a controlled manner by changing the $\mathrm{N}_{2}$ environment to introduce more nitrogen. The post-growth nitriding of RENs offers an alternative way of doping the RENs. We demonstrate post-growth doping which primarily 
allows filling of nitrogen vacancies, but it is possible that post-growth doping can introduce more nitrogen vacancies if it was performed at elevated temperature.

The RE surface has a $2 \mathrm{~nm}$ surface layer which is more reactive, and this correlates well with the size of the initial Sm islands. In the denitriding of the REN, the top $2 \mathrm{~nm}$ to $4 \mathrm{~nm}$ are available for nitrogen extraction from the lattice. This more reactive surface layer is important to characterise and manage when making REN devices, both in this thesis and for future device manufacturing.

Based on the $\mathrm{N}_{2}$ pressures at which nitriding of Sm starts and the pressures at which nitrogen escapes the surface layers in the SmN lattice we propose that the nitrogen equilibrium pressure of $\mathrm{SmN}$ is between $5 \cdot 10^{-6}$ mbar to $3.5 \cdot 10^{-5}$ mbar. This is an extremely low nitrogen equilibrium pressure [50].

\subsection{Conclusions and future work}

This chapter has presented an initial study of the reaction between RE metals and $\mathrm{N}_{2}$, with a particular focus on $\mathrm{Sm}$. To expand and further clarify the nitriding reaction many future experiments could be performed.

The obvious next step for this project would be to investigate the chemical reaction on the surface. Both theoretical studies and experimentally, for example using XPS, would aid future understanding of the cracking process. A better understanding of how, and where, the $\mathrm{N}_{2}$ cracks on the RE surface will offer insight into how to tune the growth of the RENs to control different properties.

It would be of use to understand whether the nitrogen diffuses through the RE/REN films as atomic or molecular nitrogen. Theoretical studies may offer insights into this. If the nitrogen diffuses as atomic nitrogen, the surface $\mathrm{N}$-coverage will be related to the diffusion coefficient as a free surface RE-ion is needed to crack the $\mathrm{N}_{2}$ before diffusion can occur. On the 
other hand, if the nitrogen diffuses as $\mathrm{N}_{2}$, the surface $\mathrm{N}$-coverage should not affect the diffusion much as the nitrogen does not need a free RE surface site.

To further probe the diffusion pathway it would be interesting to nitride both thicker polycrystalline RE films and epitaxial RE films. A thicker polycrystalline film would allow us to see if the RE film can only be nitrided to a certain depth, or if a thicker film changes the timescales of the nitriding. An epitaxial film should be less effective at nitrogen cracking and diffusion if these are mediated through grain boundaries. Larger grains and limited grain boundaries limit reaction rates in other materials $[59,60]$.

Many nitriding studies report significant changes in reaction and diffusion when introducing a small amount of oxygen together with the nitrogen. Replicating this in the REs would be particularly interesting as the formation of RE oxides is a common failure mode of our REN films.

As the RE surface can crack the $\mathrm{N}_{2}$ molecule, it may be possible to use a RE surface in the manufacturing of ammonia. To investigate the possibility of using the REs to make ammonia in a controlled way we would introduce hydrogen in the UHV chamber. The hydrogen could be introduced either after having nitrided the surface or together with the $\mathrm{N}_{2}$ to see if the reaction is likely to happen on the surface, or if $\mathrm{N}_{2}$ needs to get to the surface to crack first and then precipitate out of the REN layer to react with the hydrogen.

Ammonia is currently produced using the Haber-Bosch process which is inefficient and requires high pressures and temperatures during manufacturing. Substantial research effort is directed to finding a more efficient replacement process for making ammonia, and in researching the nitriding of the REs, we take a small step towards contributing to the development of new ammonia synthesising processes [67]. The possibility of using the REs to make ammonia is of interest as ammonia is required in large amounts as a precursor in fertiliser production. 
This thesis has focussed on the nitridation reaction, and as such the same setup is not ideal for studying ammonia production. A more reasonable way to study the ammonia production could include crushing the $\mathrm{RE}$, for example in a ball mill, and continuously expose fresh RE to a very clean controlled $\mathrm{N}_{2}$ and $\mathrm{H}_{2}$ atmosphere, for example in a glove box. 


\section{Chapter 5}

\section{Rare earth nitride growth}

After studying the reaction between Sm, Gd, Dy, and nitrogen gas in the previous chapter, this chapter aims to capture some properties of $\mathrm{SmN}$ and GdN. We use a growth process for the RENs which has been developed over many years, and in this chapter, we focus on properties and issues which are important when moving towards tunnel structures and device manufacturing in the next chapter. This chapter begins with studying the relationship between the conductivity of $\mathrm{SmN}$ and the growth pressure to see how we need to choose the growth conditions if we want to dope SmN. Controlled doping of SmN allows us to match conductivities of $\mathrm{SmN}$ and GdN (for which the conductivity-pressure relationship has already been investigated [2]) in a device structure. We then study the conductance evolution with the thickness of $\mathrm{SmN}$ to investigate the growth and find when the conductivity becomes stable. We then move on to an experiment with GdN where the ion exposure post-growth has been varied over a single GdN film which results in structural, magnetic, and electrical changes to the GdN. Finally, we investigate making ohmic contacts to GdN and SmN by studying current density-voltage (J-V) characteristics of SmN and GdN films contacted with $\mathrm{Au}$ and $\mathrm{Al}$ contacts. 


\subsection{Doping SmN during growth}

The conductivity of $\mathrm{GdN}$ has been established to be inversely proportional to the nitrogen flux during growth, supporting the claim of GdN being a dopable semiconductor [2]. No equivalent experiment has been published for $\mathrm{SmN}$; hence we present it here. The same UHV system and $\mathrm{N}_{2}$ pressure monitoring equipment was used in the $\mathrm{SmN}$ experiment as in the previously published GdN experiment [2].

\subsubsection{Growth}

This experiment was performed using the setup described in section 3.6.1. A substrate with predeposited Au contacts was loaded into the growth chamber, with the resistance between the contacts being monitored by a multimeter via an electrical feed-through. A thin film of $\mathrm{SmN}$ was grown with the highest $\mathrm{N}_{2}$ pressure possible in the UHV chamber, $3 \cdot 10^{-4}$ mbar. The SmN growth continued until the conductance increased linearly with thickness. The $\mathrm{N}_{2}$ flow into the UHV chamber was then turned down slightly - causing the $\mathrm{N}_{2}$ pressure to drop slightly - while the Sm deposition rate was kept constant. The lower nitrogen pressure made the next layer of the SmN film slightly more conductive due to more nitrogen vacancies being present in the film. The flow and pressure were further reduced in steps after the film had been allowed to grow for a sufficient amount of time at the current pressure. Once the $\mathrm{N}_{2}$ flow was turned down all the way to zero the shutter was closed, and the experiment finished.

The conductivity of each layer was then found by subtracting the conductance of all previous layers from the total conductance by treating the sample as a number of parallel resistors of different conductance. Since each layer corresponds to a specific $\mathrm{N}_{2}$ pressure a relationship between $\mathrm{N}_{2}$ growth pressure and conductivity could be found. 


\subsubsection{Pressure dependent conductivity of SmN}

The conductivity of $\mathrm{SmN}$ as it depends on the $\mathrm{N}_{2}$ partial pressure is plotted in fig. 5.1 together with the equivalent GdN data from Ref [2].

The conductivity of $\mathrm{SmN}$ is inversely proportional to the $\mathrm{N}_{2}$ growth pressure. Higher $\mathrm{N}_{2}$ pressure during growth corresponds to lower conductivity of the SmN film. This fits well with the argument that the main dopants are nitrogen vacancies in $\mathrm{SmN}$, as they are in GdN. The data for $\mathrm{SmN}$ overall matches the GdN conductivity data well.

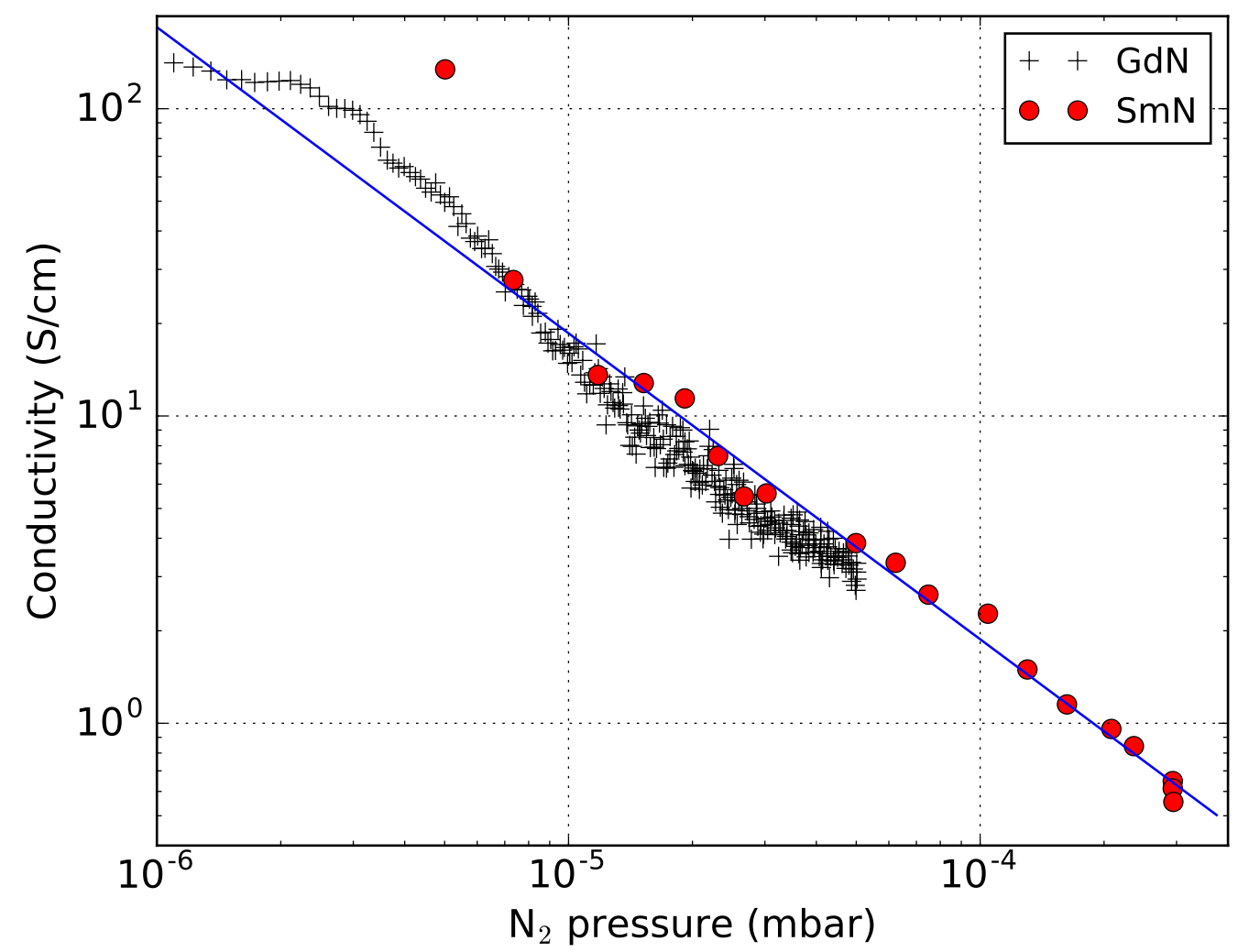

Figure 5.1: In-situ conductivity of $\mathrm{SmN}$ and $\mathrm{GdN}$ vs $\mathrm{N}_{2}$ growth pressure showing an inverse relationship in the $\mathrm{N}_{2}$ pressure range $10^{-6} \mathrm{mbar}$ to $3 \cdot 10^{-4}$ mbar. The line represents a slope of -1 . GdN data reproduced from Ref [2] with permission. 
From fig. 5.1 we can attempt to extract a reaction cross-section. At $\mathrm{N}_{2}$ pressures below $10^{-4}$ mbar the nitrogen-vacancy $\left(V_{N}\right)$ concentration rises as $P_{N}^{-1}$, immediately suggesting an enhanced reaction probability when the molecules strike a N-poor surface. The $P_{N}^{-1}$ dependence can be modelled by assuming that the probability of dissociation is zero for molecules striking a RE site with a $\mathrm{N}$ already bonded, and a finite value $\left(s_{r}\right)$ at a $\mathrm{RE}$ ion without a full set of $\mathrm{N}$ bonds, and assuming no surface mobility. Within this simple description, the number of nitrogen atoms that stick to the surface is

$$
n_{\text {stick }}=s_{r} x n_{N}
$$

where $x$ is the $V_{N}$ concentration and $n_{N}$ is the $\mathrm{N}_{2}$ flux at the surface. Each $\mathrm{N}_{2}$ molecule that hits a RE lattice surface site without a $\mathrm{N}$ bound to it will dissociate and supply one nitrogen atom with probability $s_{r}$.

The $\mathrm{N} / \mathrm{RE}$ ratio in the $\mathrm{SmN}$ layer is

$$
1-x=n_{\text {stick }} / n_{R E}=x\left[s_{r}\left(n_{N} / n_{R E}\right)\right]
$$

where $n_{R E}$ is the rare earth-atom flux. This can be rearranged to

$$
x=\frac{1}{1+s_{r} \frac{n_{n}}{n_{R E}}}=\frac{n_{R E}}{s_{r} n_{n}+n_{R E}}
$$

Since the $\mathrm{N}_{2}$ flux has to be 100 times larger than the RE flux to make a REN [30] we can simplify to

$$
x=\frac{n_{R E}}{s_{r} n_{n}}
$$

Thus for small $V_{N}$ levels (e.g. $x<0.1$ ), $x$ and the conductivity follows an inverse dependence on the $\mathrm{N}_{2}$ pressure during growth, as is observed. Further measurements of the carrier concentration in our films, coupled with assumptions that each $V_{N}$ introduces one electron into the conduction band, one finds that almost all $\mathrm{N}_{2}$ molecules striking a free $\mathrm{RE}$ atom on the surface introduces one $\mathrm{N}$ atom into the network, i.e. $s_{r} \approx 1$. The reaction cross section is large. 
If we change the nitrogen pressure or Sm flux, we will change the conductivity of the resulting $\mathrm{SmN}$ film because of the resulting change in $V_{N}$.

\subsection{Conductance evolution of $\mathrm{SmN}$}

In this section, we discuss how the conductance changes with the thickness of a SmN film during the growth. The experiment was set up according to the procedure described in section 3.6.1.

The conductance as a function of the thickness of a SmN film can be seen in fig. 5.2. The first thing to note is that the conductance is quite low, $<5 \mu \mathrm{S}$. The conductance is around three orders of magnitude lower than for the equivalent $\mathrm{Sm}$ growth experiment as described in section 4.2.2.

The conductance of the $\mathrm{SmN}$ film becomes non-zero when $3 \mathrm{~nm}$ of film growth has been recorded by the crystal balance, suggesting that the percolation threshold for the film has been reached. Once the film starts to conduct the conductance does not increase linearly with thickness until a certain thickness has been reached. The non-uniform initial conductivity suggests that similar to the Sm films in section 4.2.2, the SmN films grow in Volmer-Weber growth, which is common for polycrystalline films. SmN grown at ambient temperature grows in a polycrystalline fashion [24], which is further supported by this data.

The SmN film does not reach a constant linear conductance increase regime until around $20 \mathrm{~nm}$ thick. This suggests that we should grow SmN films to at least $20 \mathrm{~nm}$ thick during ambient temperature growth to get a continuous film. Thinner films risk not having an even $\mathrm{SmN}$ film which could lead to trouble particularly when making multi-layer devices and performing electrical measurements where the current is perpendicular to the film plane in vertical transport measurements. 


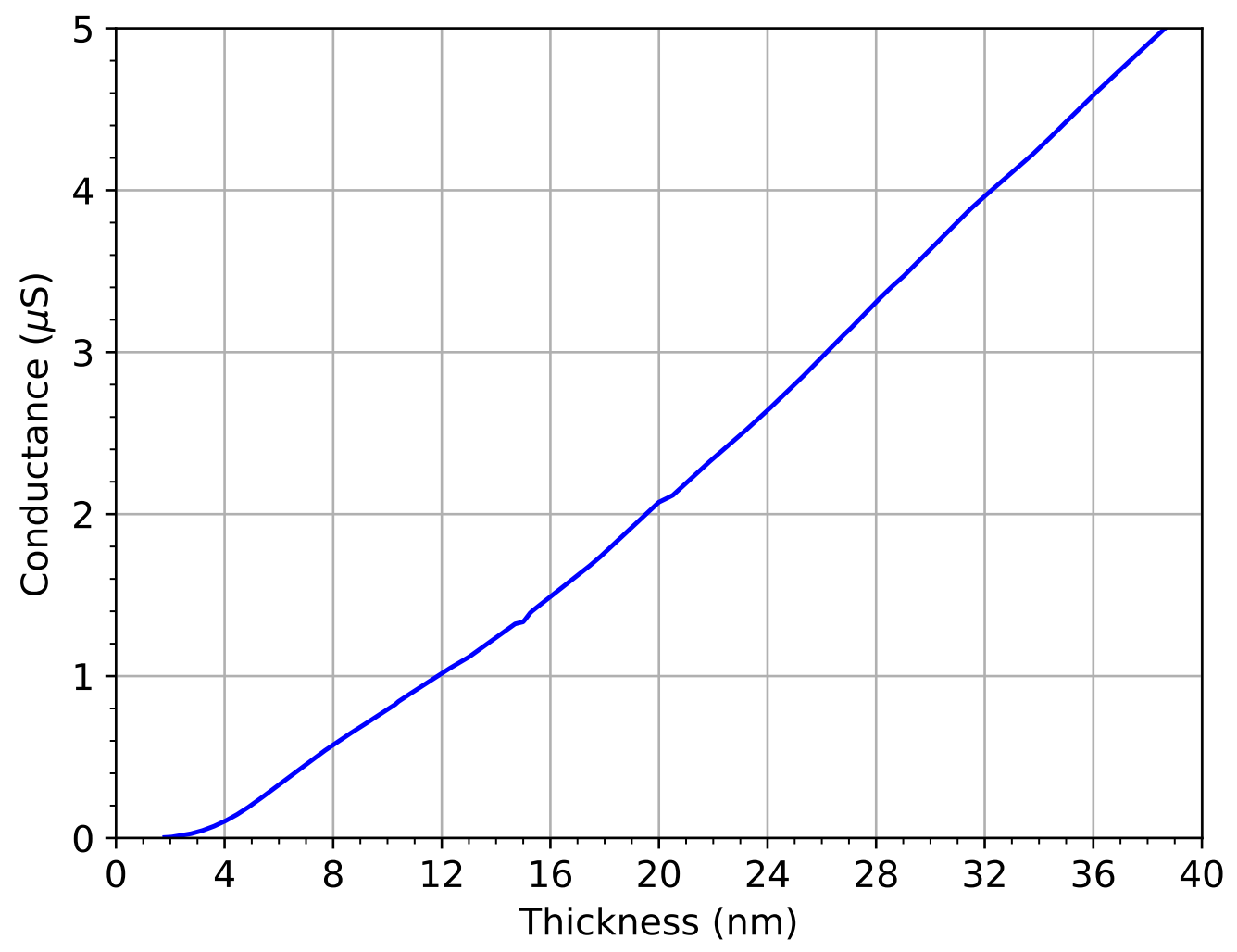

Figure 5.2: In-situ conductance vs thickness for a SmN film grown in $1.3 \cdot 10^{-4}$ mbar of $\mathrm{N}_{2}$. 


\subsection{Sample changes under ion exposure}

This section shows results from a single growth of $\mathrm{GdN}$ where half of the film was capped with $\mathrm{Al}$ and the other half with AlN. We will see that the addition of a capping layer can change the properties of the underlying REN layer depending on whether nitrogen ions are used during the capping layer growth or not.

\subsubsection{Growth}

The GdN sample is grown on a 2 inch wafer patterned in the vertical transport structure, as described in section 3.5, but modified to also have inplane electrical measurements possible by the addition of predeposited $\mathrm{Au}$ contacts in van der Pauw geometry on the bottom $10 \mathrm{~mm} \times 10 \mathrm{~mm}$ plain film areas. A schematic of the finished wafer is shown in fig. 5.3.

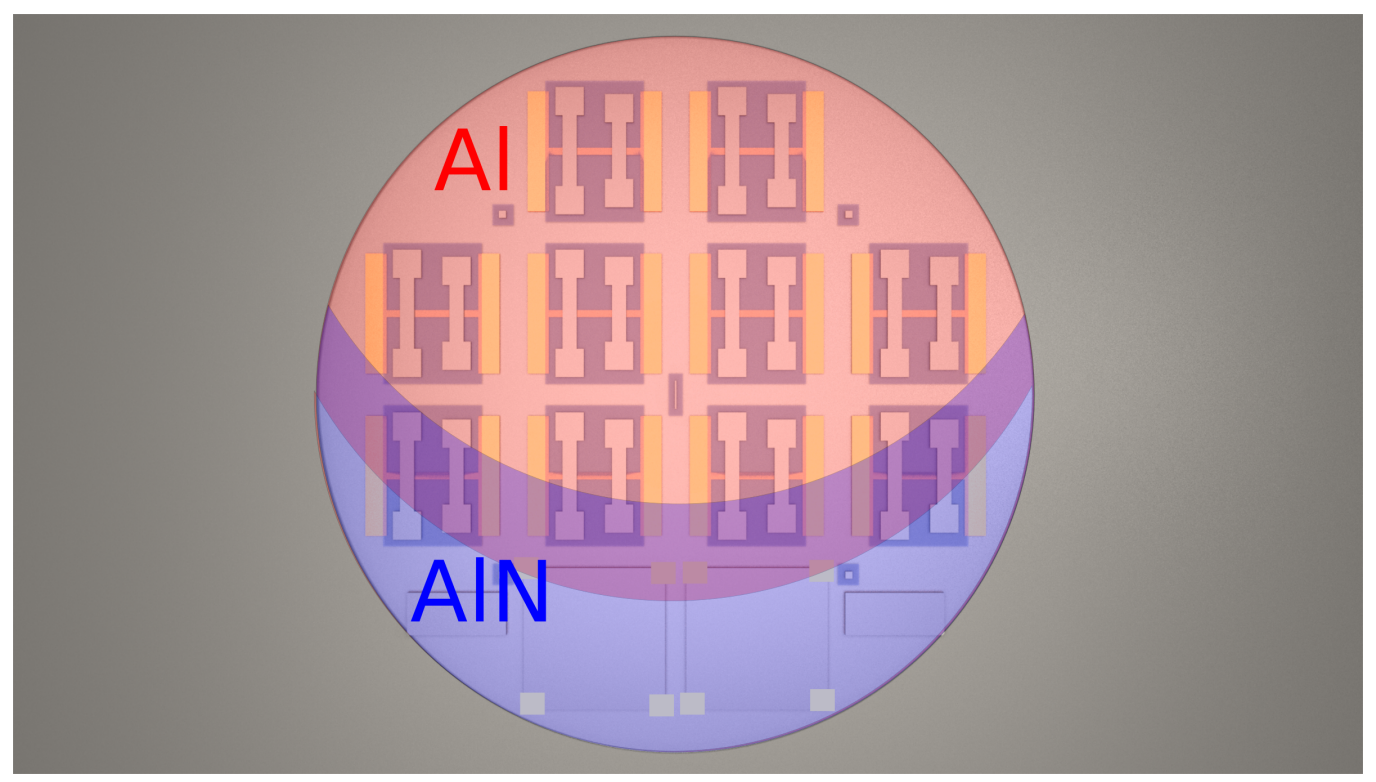

Figure 5.3: Schematic of the $\mathrm{Al}$ and AlN capped sections of the sample.

The GdN film is grown with the substrate held at stable room temperature using water cooling. The $\mathrm{N}_{2}$ pressure was $1 \cdot 10^{-4} \mathrm{mbar}$ and a $\mathrm{Gd}$ 
deposition rate of $400 \mathrm{~nm} / \mathrm{h}$. The $\mathrm{GdN}$ is grown thick ( $450 \mathrm{~nm}$ ) to ensure that a measurable resistance is derived in the out-of-plane measurements.

Once the GdN film is grown over the entire substrate, the bottom part of the sample is partially masked off using the sample shutter. This allows the top of the sample (red in fig. 5.3) to be capped with a $50 \mathrm{~nm}$ layer of Al. The sample is then rotated $180^{\circ}$ and $50 \mathrm{~nm}$ of AlN is grown on the bottom part of the sample (blue in fig. 5.3). The $\mathrm{Al}$ is deposited from an egun, and the nitrogen ions are generated by a Kaufmann EH200 ion source at $0.2 \mathrm{~A}$ emission current (standard settings for III-nitride growth in the Thermionics UHV system). The distribution of the capping layers over the surface can be seen in fig. 5.3. During the AlN growth, the $10 \mathrm{~mm}$ closest to the shutter edge ended up Al-rich (presumably due to the shutter partially blocking the $\mathrm{N}$-ion flux), prohibiting four-point resistivity measurements of the AlN capped GdN using the pre-deposited contacts but still allowing two-point resistivity measurements.

\subsubsection{Structural changes from ion exposure}

The XRD spectra of the GdN film with the two different capping layers is plotted in fig. 5.4, with a magnification of the data between $28^{\circ}$ to $35^{\circ}$ seen in fig. 5.5. Aligning the two data sets to the Si(400) peak at $69.17^{\circ}$ gives excellent alignment of the forbidden $\mathrm{Si}(200)$ peaks at $33^{\circ}$ as well, indicating that the data sets are well aligned throughout the measurement with no compensation for off-axis sample positioning being required.

As is common in polycrystalline REN films, the (111) peak around $31^{\circ}$ is the dominant peak, and since there are no other peaks close by to interfere with the intensity, we will focus on this peak for our analysis. The (222) peak shows the same behaviour as the (111) peak but is partially obstructed by a background peak which makes it less suitable for quantitative analysis.

In fig. 5.5 the data sets from fig. 5.4 have had the background removed 


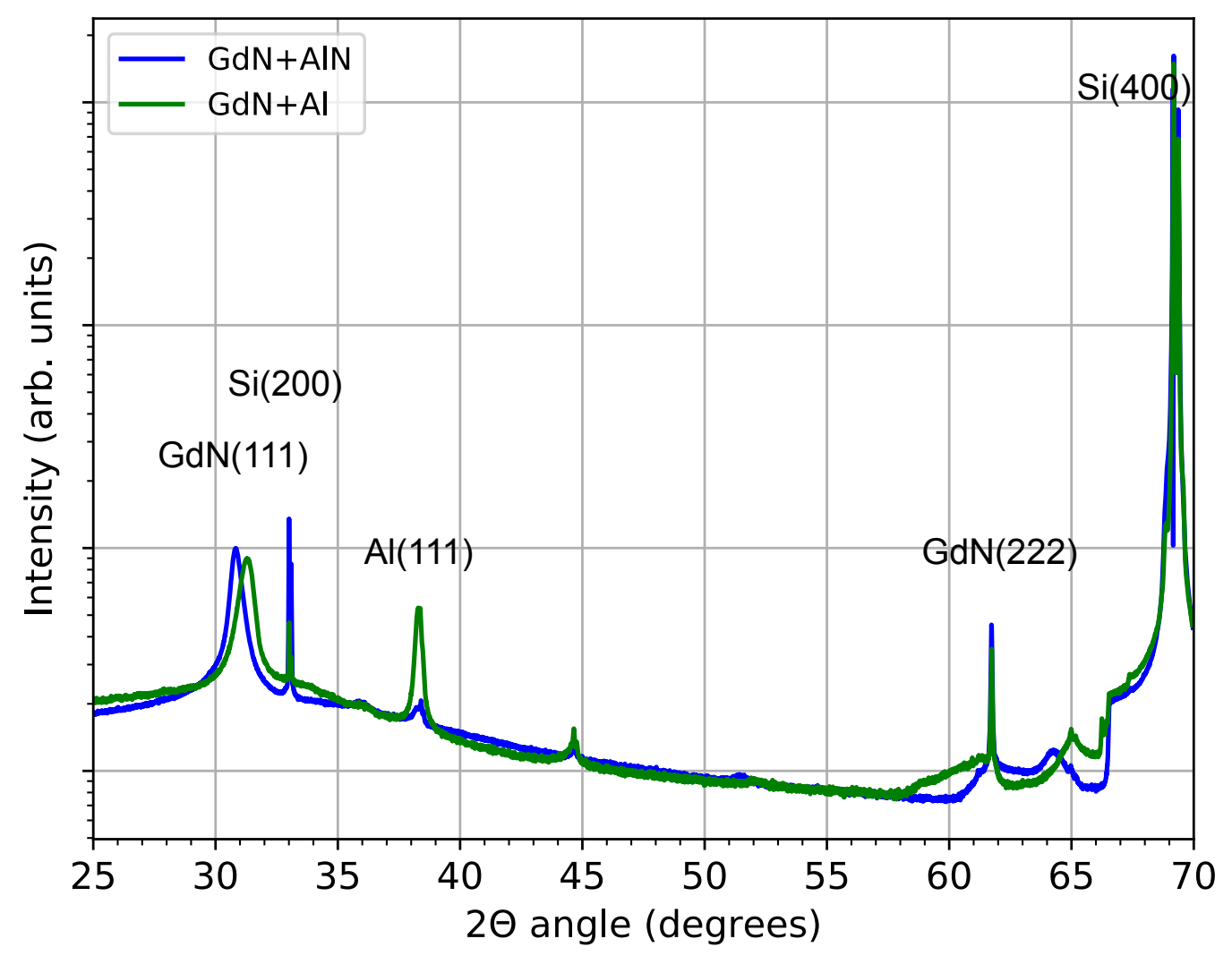

Figure 5.4: XRD spectra of GdN capped with AlN and Al. Aligned using $\mathrm{Si}(400)$ peaks. Unlabelled peaks are from the substrate or mounting mechanism.

and scaled by the Si (400) peak intensity in each data set. The GdN(111) peaks with different capping layers are shifted from each other. The GdN(111) peak of the $\mathrm{Al}$ capped $\mathrm{GdN}$ is located at $31.3^{\circ}$ which corresponds to an out-of-plane lattice parameter of $4.94 \AA$, smaller than the literature value of $4.98 \AA$ [1]. As a contrast, the GdN(111) peak of the AlN capped GdN is located at $30.8^{\circ}$ which corresponds to a lattice parameter of $5.02 \AA$. The difference in lattice parameter means the AlN capped GdN is expanded by $1.6 \%$ compared to the Al capped GdN despite the initial GdN film being identical. This observation supports a structural change induced by the nitrogen ions used when growing the capping layer. 


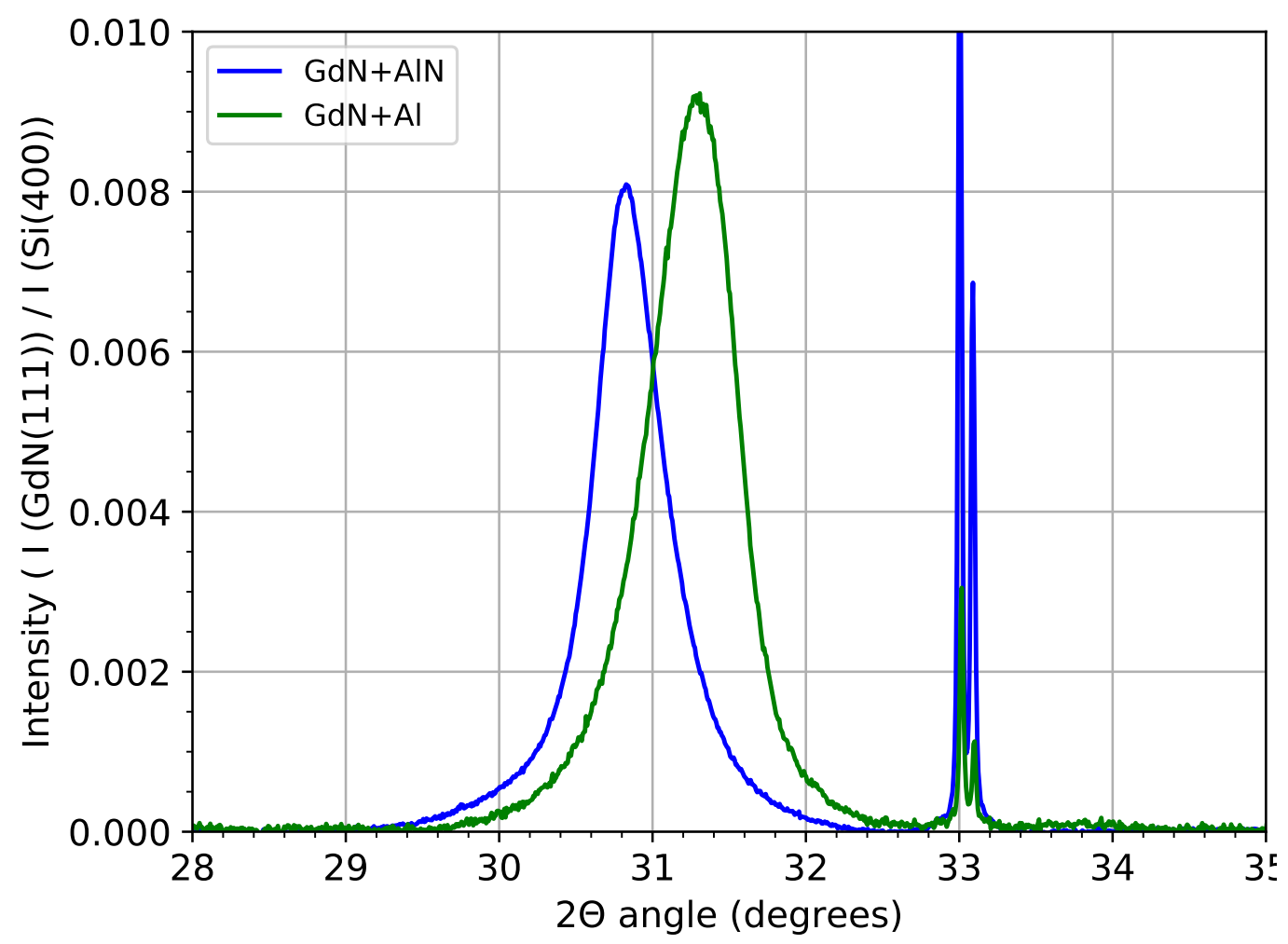

Figure 5.5: XRD spectra of GdN capped with AlN and GdN capped with Al. Maginification around the GdN (111) peak. Background removed and plotted as a fraction of the $\mathrm{Si}(400)$ peak intensity.

The intensity of the $\mathrm{GdN}(111)$ peak compared to the $\mathrm{Si}(400)$ peak in the two samples has also changed. The $\mathrm{Al}$ capped $\mathrm{GdN}$ has a $12 \%$ larger peak ratio than the AlN capped GdN.

To further explore the two $\mathrm{GdN}(111)$ peaks we fit each data set to a pseudo-Voigt model. A pseudo-Voigt fit is a combination of a Gaussian and Lorentzian line shape commonly used in fitting XRD profiles [68]. The AlN capped GdN can be seen in fig. 5.6 and the Al capped GdN can be seen in fig. 5.7. Neither data set can be fully fitted with a single pseudoVoigt profile, but both can be well fitted using a double pseudo-Voigt profile with the two profiles centred at $30.8^{\circ}$ and $31.3^{\circ}$. 


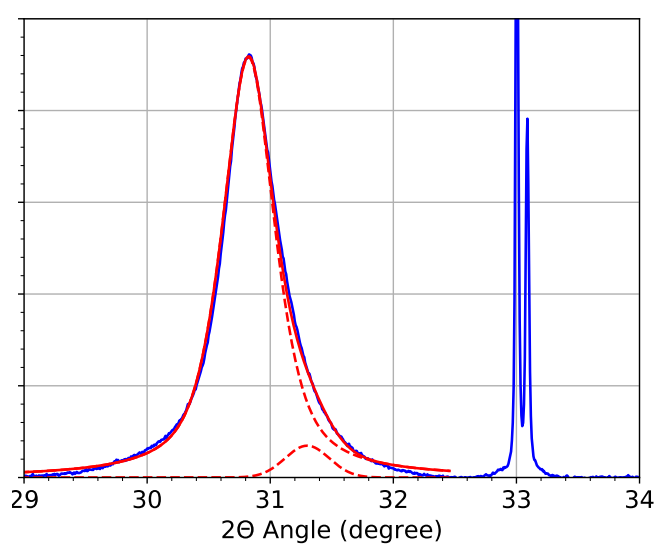

Figure 5.6: XRD spectrum of the GdN(111) peak of the AlN capped GdN with two Pseudo-Voigt models fitted (dashed line) and the combined model (solid line).

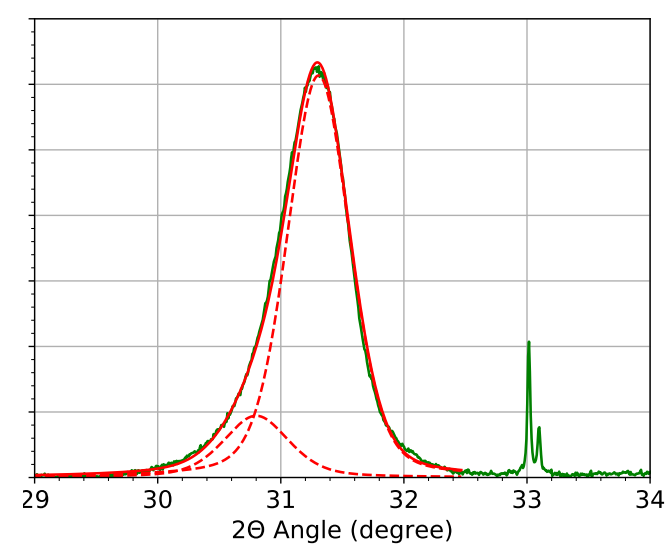

Figure 5.7: XRD spectrum of the GdN(111) peak of the Al capped GdN with two Pseudo-Voigt models fitted (dashed line) and the combined model (solid line).

The excellent fit to a double profile with identical centre positions but with different intensities for the two samples demonstrates that there are two GdN(111) phases, both of which are present in each sample in different concentrations. This observation bears large similarities to Ref [69] where two phases of $\mathrm{GdN}$ are observed when varying the sputtering power and nitrogen pressure for different GdN growths. Ref [69] find an expanded phase of $\mathrm{GdN}(111)$ which increases in intensity as the sputtering power during growth is increased in contrast to our observations that the expanded phase arises from the ions during the capping layer growth.

The lattice parameters observed by Ref [69] are larger (5.02 $\AA$ to $5.12 \AA$ ) than the lattice parameters for the $G \mathrm{dN}(111)$ in this chapter $(4.94 \AA$ to $5.02 \AA)$, suggesting that there may even be three different structural phases attributed to GdN. The $5.02 \AA$ phase is found both in this work, as the expanded phase (relative to the non-ion exposed GdN), and in Ref [69] as the non-expanded phase (relative to the even more ion exposed phase). It is worth noting that all samples reported in Ref [69] are grown using reac- 
tive magnetron sputtering where the $\mathrm{GdN}$ is grown with ionised nitrogen while all the GdN reported here is grown with thermal Gd deposition in a molecular nitrogen atmosphere with or without exposure to nitrogen ions during the capping layer growth. The larger lattice parameters found during growth with nitrogen ions suggests that the ions are responsible for the lattice change in our experiments as well.

In the AlN capped GdN the expanded phase has $95 \%$ of the total intensity whereas in the Al capped GdN the expanded lattice phase has $15 \%$ of the total intensity. The relative intensity of the two phases is insensitive to how the background subtraction was chosen, and how much of the peak broadening is attributed to instrumental broadening.

The increase in the relative intensity of the expanded phase and decrease in the intensity of the un-expanded phase when the GdN is capped with AlN demonstrates that the process of growing the AlN changes the crystal structure of the GdN. Thinner GdN films ( 100 nm) capped with AlN or GaN show excellent fit to a single pseudo-Voigt profile centred at $30.8^{\circ}$ - the expanded phase (data not shown). This suggests that the unexpanded phase we see in our thicker AlN capped GdN sample is at the bottom of the GdN layer, protected by the bulk of the GdN film from ion bombardment.

In conclusion, despite the initial GdN film being identical across the substrate the use of $\mathrm{AlN}$ as a capping layer instead of $\mathrm{Al}$ reduces the $\mathrm{GdN}(111)$ intensity, and changes the relative distribution of the un-expanded and expanded GdN(111) phases present in the GdN lattice. The change in structure from the ion bombardment causes drastic changes to the magnetic and electrical properties of the GdN film which we will discuss below. 


\subsubsection{Magnetic changes from ion exposure}

The magnetic properties of a material correlate strongly with their structural properties. Hence, we measure the magnetic moment as a function of temperature and applied field for the GdN sample with the two different capping layers to investigate whether the change in structure can be seen to affect the magnetic moment.

The magnetic moment as a function of applied field for the two different samples at two temperatures, $2 \mathrm{~K}$ and $20 \mathrm{~K}$ is shown in fig. 5.8. The two curves from the $\mathrm{Al}$ capped $\mathrm{GdN}$ show a saturating magnetic moment at high applied fields, whereas the AlN capped GdN does not appear to saturate the magnetisation even at $7 \mathrm{~T}$. The Al capped GdN curves become colinear at higher fields, indicating that they are indeed saturated, but that a smaller percentage of the film is fully magnetised at $20 \mathrm{~K}$ than at $2 \mathrm{~K}$. The AlN capped GdN curves do not appear to have become colinear even at $7 \mathrm{~T}$.

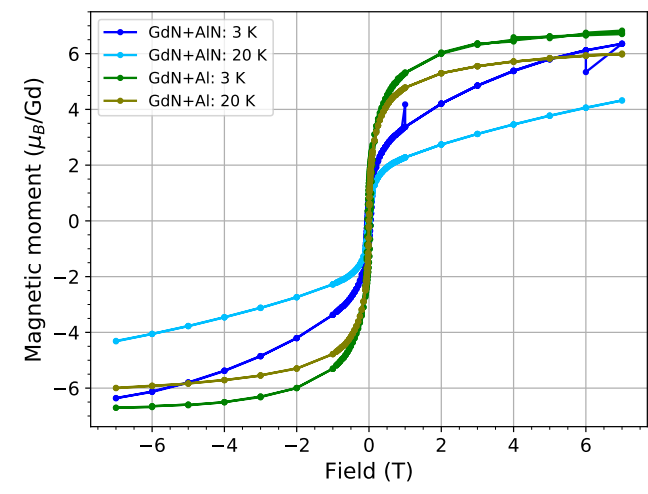

Figure 5.8: Magnetic moment hysteresis loops between $\pm 7 \mathrm{~T}$ for AlN- and Al-capped GdN at $2 \mathrm{~K}$ and $20 \mathrm{~K}$.

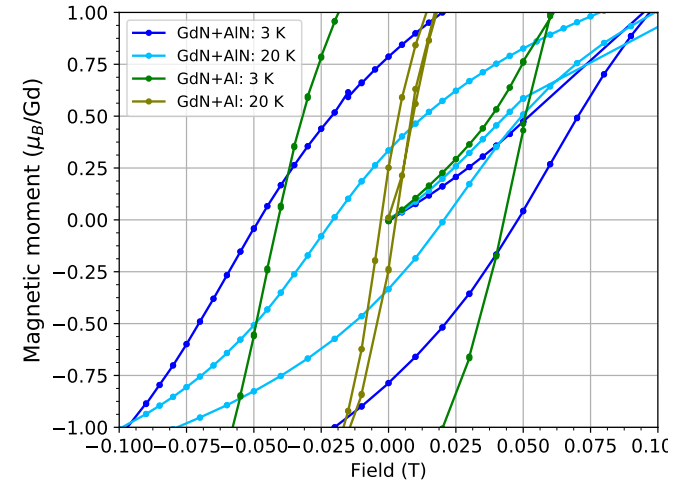

Figure 5.9: Magnification between $\pm 0.1 \mathrm{~T}$ of the magnetic moment hysteresis loops between $\pm 7 \mathrm{~T}$ for AlN- and Al-capped GdN at $2 \mathrm{~K}$ and $20 \mathrm{~K}$.

The Al capped GdN saturates close to $7 \mu_{\mathrm{B}} / \mathrm{Gd}^{3+}$ ion at $2 \mathrm{~K}$, which cor- 
responds well to literature values of the GdN saturation magnetisation. The AlN capped GdN has a lower magnetic moment at $7 \mathrm{~T}$, particularly when comparing the $20 \mathrm{~K}$ curves, but since the curves have not saturated, it is not possible to extract what the saturation magnetisation would be. A perfect GdN sample should saturate at a low applied field to $7 \mu_{\mathrm{B}} / \mathrm{Gd}^{3+}$ ion. The sharper saturation of the Al capped GdN likely indicates that it is closer to perfect GdN than the AlN capped GdN.

The coercive field for the four curves is plotted in fig. 5.9, which is just a low field magnification of fig. 5.8. The coercive field for the Al capped sample is smaller than the AlN capped sample, particularly at $20 \mathrm{~K}$.

The remanence magnetisation in the $\mathrm{Al}$ capped sample is about twice the remanence of the AlN capped sample at $3 \mathrm{~K}$, while the remanence is marginally larger in the AlN capped sample than in the Al capped sample at $20 \mathrm{~K}$.

To further explore the magnetic properties we study the zero-fieldcooled and field-cooled magnetisation as a function of temperature for the two samples, seen in fig. 5.10. The corresponding inverse susceptibility is plotted in fig. 5.11. The Al capped GdN show typical ZFC, FC, and $1 / \chi$ with a Curie-Weiss paramagnetism model predicting $T_{C}=62 \mathrm{~K}$. In contrast, the AlN capped GdN shows a weak double magnetic transition with the first transition at $T_{C}=62 \mathrm{~K}$ and a second centred around $50 \mathrm{~K}$. The additional transition at $50 \mathrm{~K}$ has previously been reported for epitaxial GdN samples. [29]

Remembering that the Al capped and AlN capped GdN show two different lattice parameters we can reasonably attribute the strong $62 \mathrm{~K}$ transition to the non-expanded phase with a lattice parameter of $4.94 \AA$, the change in crystal structure has a corresponding change in magnetic response.

The observed change in magnetic properties for the different structure phases corresponds well to findings from Ref [34] where a $2.4 \%$ lattice expansion caused a similar change to the magnetic hysteresis curves, 
where the expanded lattice arises from an ion-assisted growth rather than a molecular nitrogen growth. The previously discussed expanded GdN(111) phase reported in Ref [69] is found to be anti-ferromagnetic. The expanded GdN phase in Ref [69] has a larger lattice parameter than any of our GdN films, and may even be a third phase of $\mathrm{GdN}$ as discussed above originating from the higher sputtering powers used. We have not found evidence of anti-ferromagnetism in our GdN films, supporting the idea that the expanded GdN phase in Ref [69] is a third GdN phase.

We attribute the magnetic transition around $50 \mathrm{~K}$ to the expanded $\mathrm{GdN}(111)$ phase, while the $62 \mathrm{~K}$ transition arises from the unexpanded $\mathrm{GdN}(111)$ phase. From the measurements of the field-dependent magnetisation, we find that the expanded $\mathrm{GdN}(111)$ phase has a weaker magnetic response at fields below $8 \mathrm{~T}$. Being aware that the expanded phase has a different magnetic response, in particular, a larger coercive field is important when making memory devices using GdN.

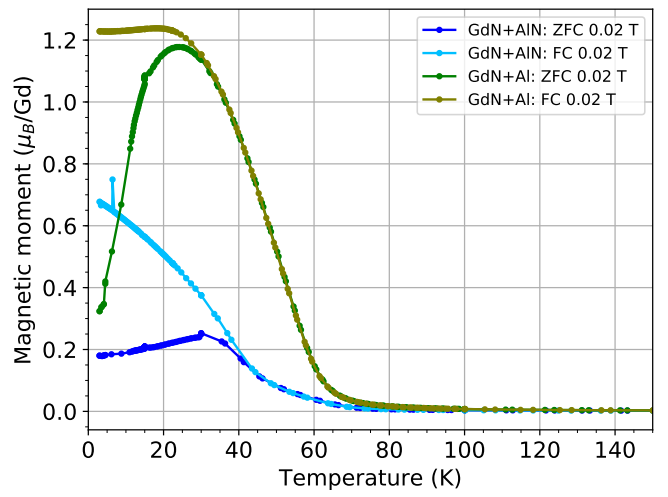

Figure 5.10: Magnetic moment vs temperature in ZFC and FC measurements in $0.02 \mathrm{~T}$ for both AlNand Al-capped GdN.

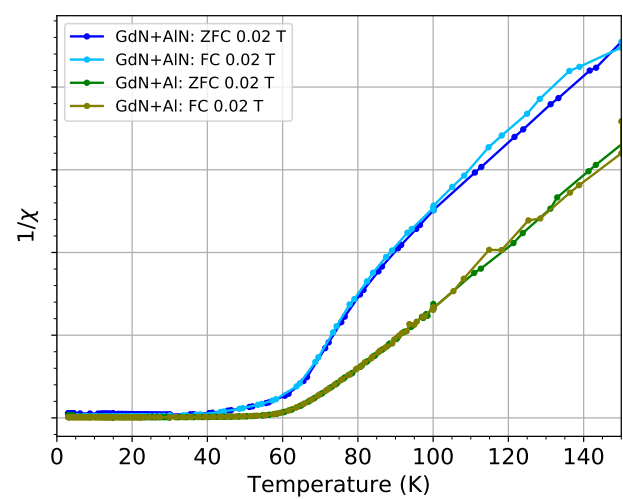

Figure 5.11: $1 / \chi$ vs temperature for ZFC and FC measurements in $0.02 \mathrm{~T}$ for both AlN- and Al-capped GdN. 


\subsubsection{Electrical changes from ion exposure}

The electrical resistivity of the AlN capped and Al capped GdN films is shown in table 5.1. The AlN capped GdN is measured with the current parallel to the film plane and the resistance measured is high compared to the $\mathrm{Al}$ capped $\mathrm{GdN}(\mathrm{R}(300 \mathrm{~K})=18 \mathrm{k} \Omega)$. The $\mathrm{Al}$ capped $\mathrm{GdN}$ is measured with current perpendicular to the film plane, and the measured resistance is comparatively low $(\mathrm{R}(300 \mathrm{~K})=0.4 \Omega)$. We cannot rule out that any differences in the electrical behaviour arise from the difference in measurement orientation as the films may not be identical when looking in-plane and out-of-plane, but the differences are nonetheless important to discuss.

The room temperature resistivity is comparable for the differently capped samples, and comparable to many room temperature resistivities of GdN reported in the literature. [1] The Al capped GdN show no increase in resistivity when measured at $4 \mathrm{~K}$ when compared to the resistivity at $300 \mathrm{~K}$, while the AlN capped GdN show a 100-fold increase in resistivity when going to $4 \mathrm{~K}$, reaching a resistance unmeasurable in any characterisation equipment available in the labs. Both samples show a characteristic GdN resistance anomaly around $50 \mathrm{~K}$ (not shown).

\begin{tabular}{lll} 
& $\mathbf{G d N}+\mathbf{A l N}$ & $\mathbf{G d N}+\mathbf{A} \mathbf{1}$ \\
\hline$\rho(300 \mathrm{~K})$ & $1.6 \Omega \mathrm{cm}$ & $1.2 \Omega \mathrm{cm}$ \\
$\rho(4 \mathrm{~K})$ & $>100 \Omega \mathrm{cm}$ & $1.2 \Omega \mathrm{cm}$
\end{tabular}

Table 5.1: Resistivity of identical GdN films capped by AlN and Al measured with current parallel and perpendicular to the film plane, respectively, at $300 \mathrm{~K}$ and $4 \mathrm{~K}$.

\subsection{Ohmic contacts to REN films}

To make devices using the rare earth nitrides we need to be able to make electrical contact with the RENs reliably. This section shows current-voltage 
characteristics of $\mathrm{SmN}$ and $\mathrm{GdN}$ in vertical transport mode with contacts of different metals being used. All samples in this section have been manufactured in the vertical transport structure as described in section 3.5, and all samples have an Au bottom contact which we already know makes ohmic contact to GdN [70].

When making and measuring samples in vertical transport mode extra care must be taken. In a thin film on the order of $100 \mathrm{~nm}$ thick, the series resistance of the thin film is small compared to typical in-plane measurements. A small series resistance of the REN thin film means we could be measuring an artificially high resistance as resistance contributions from contacts can contribute significantly if the contact layers are thin or resistive. To minimise resistance contributions from the contacts they are made to be of a comparable thickness to the REN film.

The current density-voltage characteristics of a SmN sample at different temperatures are shown in fig. 5.12. The $\mathrm{SmN}$ is capped with a thick $\mathrm{Al}$ film. The J-V curves in fig. 5.12 are linear at all temperatures, indicating an ohmic junction. The maximum current through the sample is $1 \mathrm{~mA}$, which corresponds to a voltage of $35 \mu \mathrm{V}$ to $80 \mu \mathrm{V}$ at different temperatures. These values, in combination with the sample dimensions, gives a resistivity of $56 \mathrm{~m} \Omega \mathrm{cm}$ to $130 \mathrm{~m} \Omega \mathrm{cm}$ for the $\mathrm{SmN}$ at different temperatures. The resistivity is comparable to previously reported in-plane $\mathrm{SmN}$ resistivities $[39,71]$. It is worth noting that this $\mathrm{SmN}$ film shows a positive temperature coefficient of resistance, suggesting it is doped to degeneracy. The positive temperature coefficent in the RENs has been reported previously in GdN [70].

The current-voltage characteristics of a GdN sample at different temperatures of a GdN sample are shown in fig. 5.13. The GdN is capped with a thick $\mathrm{Al}$ film. The maximum current through the sample is $1 \mathrm{~mA}$, which corresponds to a voltage of $\sim 3.5 \mathrm{mV}$ at all temperatures, with a small difference around $50 \mathrm{~K}$. The measured current and voltage, in combination with the sample dimensions, gives a resistivity of $1.2 \Omega \mathrm{cm}$ for the GdN. 


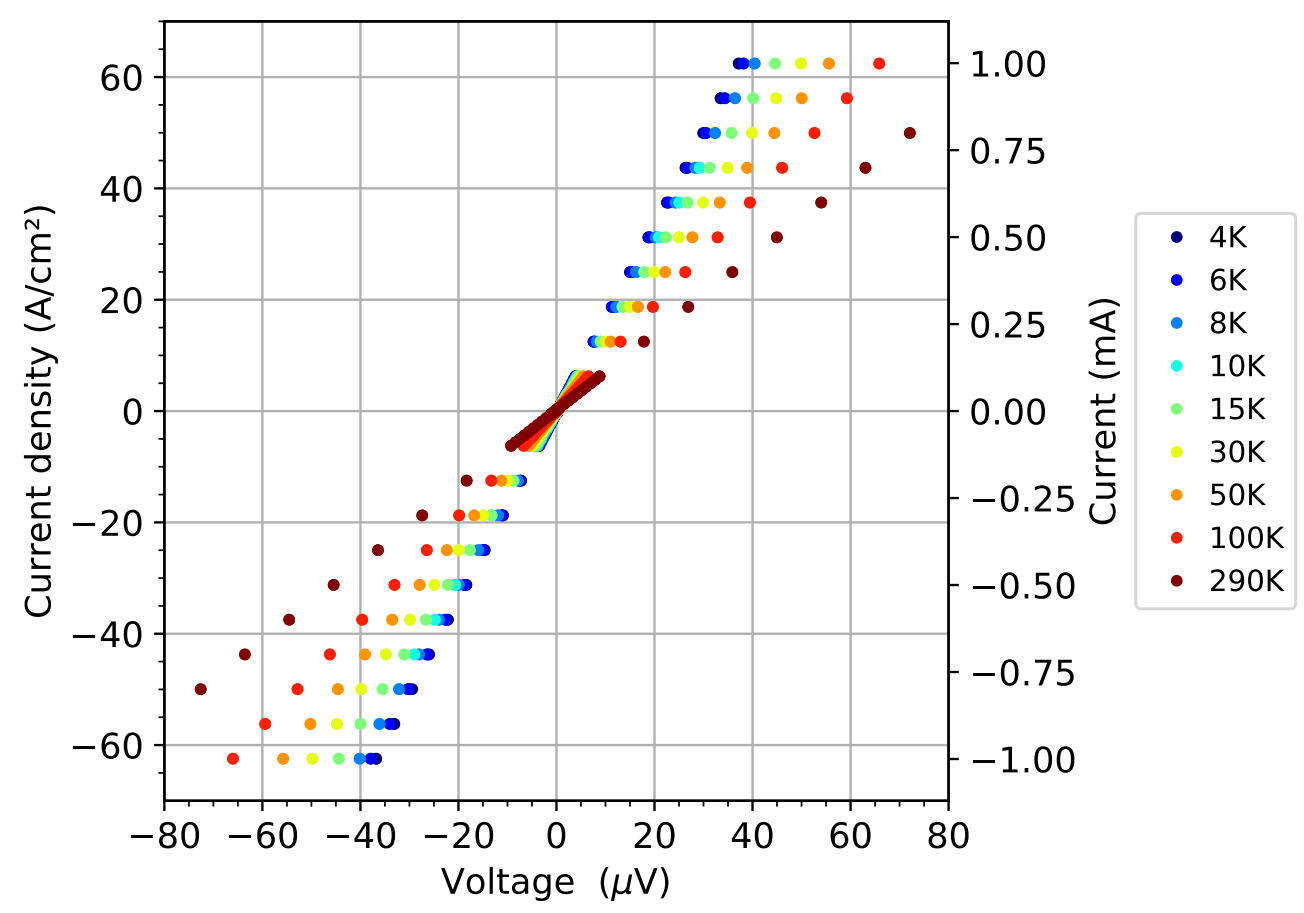

Figure 5.12: J-V curves at different temperatures of a $\mathrm{SmN}$ film contacts with $\mathrm{Au}$ (bottom) and $\mathrm{Al}$ (top).

The resistivity is comparable to previously reported in-plane GdN resistivities. [1,72] Note that this GdN sample does display a small anomaly around $T_{C}$ as expected, but this is not easily observed from the J-V characteristics.

The linear J-V characteristics and the comparable resistivities to inplane measurements of $\mathrm{SmN}$ and $\mathrm{GdN}$ demonstrates that $\mathrm{Au}$ and $\mathrm{Al}$ make ohmic contacts to $\mathrm{GdN}$ and $\mathrm{SmN}$ in transport perpendicular to the film plane. Both $\mathrm{Au}$ and $\mathrm{Al}$ are therefore suitable for use as contact metals in future device fabrication. Previous contact metal investigations by Ref [73] agree that $\mathrm{Al}$ and $\mathrm{Au}$ make ohmic contact to $\mathrm{GdN}$ while $\mathrm{Gd}$ and $\mathrm{Nb}$ contacts to GdN produce a small Schottky barrier. However, the small 


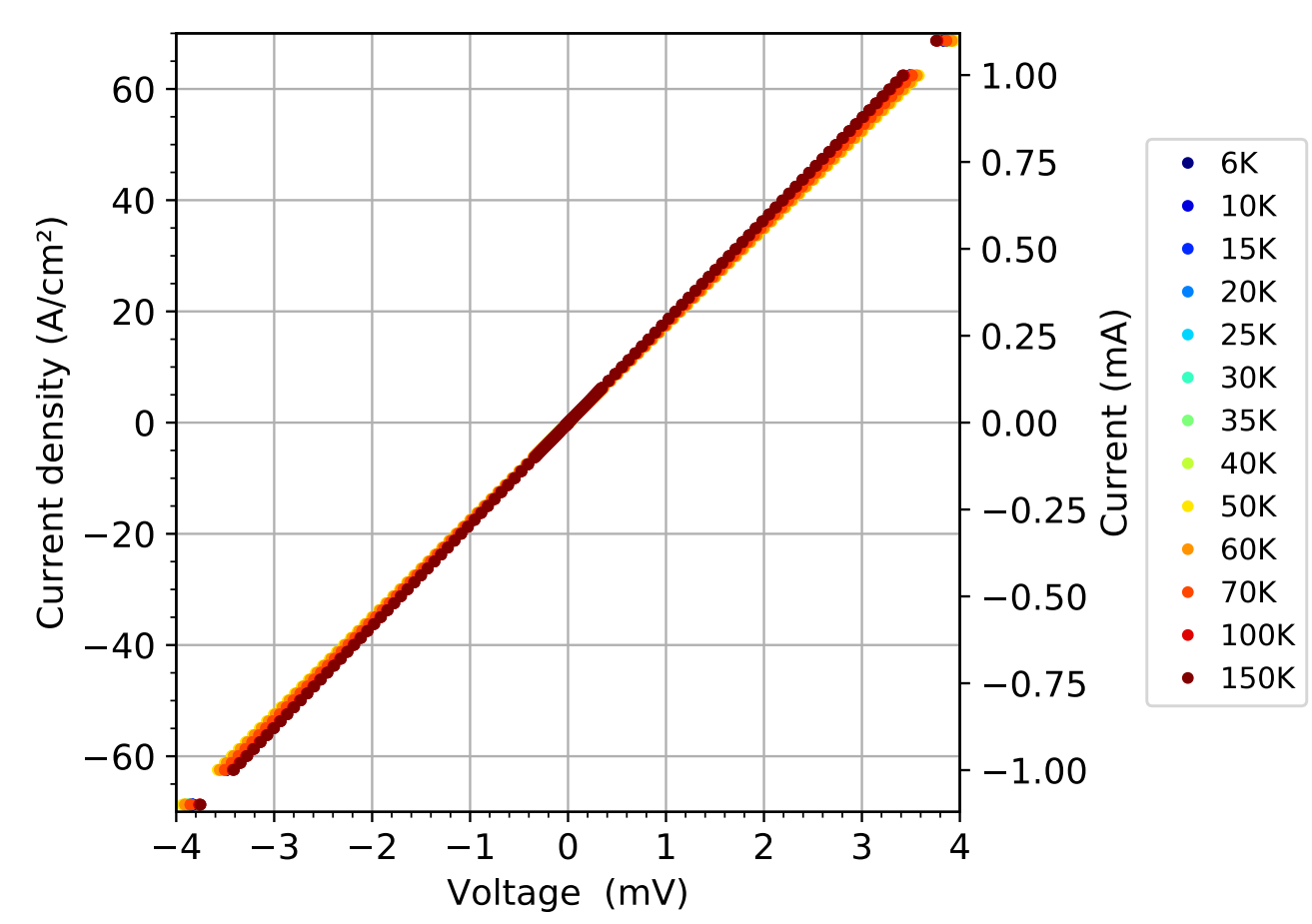

Figure 5.13: J-V curves at different temperatures of a GdN film contacts with $\mathrm{Au}$ (bottom) and $\mathrm{Al}$ (top).

Schottky barrier with GdN from $\mathrm{Gd}$ and $\mathrm{Nb}$ is not found to measurably influence the behaviour of GdN tunnel devices [73].

\subsection{Discussion}

The appearance of two separate GdN(111) phases in the XRD spectrum, as opposed to a single phase or a distribution of many different phases, suggest that GdN has two prefered (111) crystal structures with slightly different lattice parameters, and correspondingly different magnetic and electrical properties. The $4.94 \AA$ phase has a lower resistivity, sharper saturation of the magnetisation, and $\mathrm{T}_{C}=62 \mathrm{~K}$. The $5.02 \AA$ phase has higher 
resistivity, slow saturation of the magnetisation, and $\mathrm{T}_{C}=50 \mathrm{~K}$.

The interpretation of two GdN phases corresponds well with findings from Ref [34] where a $2.4 \%$ lattice expansion caused a similar change to the magnetic hysteresis curves, where the expanded lattice arises from an ion-assisted growth rather than a molecular nitrogen growth. Similarly, the appearance of expanded $\mathrm{GdN}(111)$ phase in Ref [69] with changing $\mathrm{N}_{2}$ pressure and sputtering power during GdN growth, as mentioned before, also changes the magnetic behaviour but the expanded phase is found to be anti-ferromagnetic which we have not found evidence for in our films. As the fraction of the expanded GdN phase increases the $\mathrm{T}_{C}$ of the resulting GdN was found to increase [69]. Ref [29] attribute a ferromagnetic transition at $50 \mathrm{~K}$ in $\mathrm{GdN}$ to intrinsic $\mathrm{GdN}$ while a $70 \mathrm{~K}$ ferromagnetic transition in $\mathrm{GdN}$ is mediated by magnetic polarons at the nitrogen-vacancy sites.

The finding that the nitrogen conditions during the capping layer growth can affect the film agrees well with the findings in the previous chapter, where the conductance of the $\mathrm{SmN}$ was altered post-growth with higher nitrogen pressure. The similarities in the conductivity - pressure dependence for SmN and GdN presented in fig. 5.1 together with the ability to dope $\mathrm{SmN}$ post-growth described in the previous chapter suggest that we may be able to observe two (111) phases in SmN as well. The 'true' properties of GdN and SmN are highly discussed in the literature, with different groups finding different behaviours and theoretical predictions only corresponding to behaviours of RENs from specific preparation methods. If the GdN and SmN form different structural phases with different behaviour depending on preparation conditions, this may explain a large portion of the differences between published results. 


\subsection{Conclusions and future work}

In this chapter, we have discussed a number of phenomena in the RENs, all of which are relevant in making devices.

We showed that the conductivity of $\mathrm{SmN}$ is inversely proportional the nitrogen partial pressure during growth, just as for GdN. From this, we calculated a sticking coefficient of nitrogen to rare earth atoms close to one. Knowing the dependence of conductivity on pressure is important in GdN/SmN device manufacturing as it allows us to tune the conductivity of both layers by manipulating the growth pressure.

We also showed the in-situ conductance evolution of SmN with increasing film thickness, and concluded that a polycrystalline SmN film has to be at least $15 \mathrm{~nm}$ to $20 \mathrm{~nm}$ thick to get a film of consistent conductivity. A varying conductivity suggests that the films are not of uniform thickness until the thicknesses are above $15 \mathrm{~nm}$ to $20 \mathrm{~nm}$. The thickness required for a uniform film will, of course, depend on the roughness of the substrate, where a rougher layer under the $\mathrm{SmN}$ may require a thicker $\mathrm{SmN}$ to get a consistent film. The minimum thickness for a uniform film is important in device structures where the $\mathrm{SmN}$ is likely grown on one or multiple previous layers.

We showed how a GdN film changes when it is exposed to ions. The ion-exposed GdN lattice expands by $1.6 \%$, the magnetic moment lowers and does not reach saturation even at $7 \mathrm{~T}$, and the resistivity increases by more than two orders of magnitude at low temperatures. These findings mean that caution is required when growing and comparing different REN films, particularly those with different capping layers. It also means that growing devices with tunnel barriers made using nitrogen ions we risk changing the surface of the REN it is deposited onto by exposing it to the nitrogen ions. The surface of ion-exposed GdN shows a more slowly saturating magnetisation, a larger coercive field, and a higher resistivity than non-ion-exposed GdN. 
The change in GdN with nitrogen ion exposure suggest a thorough investigation into how the GdN properties change with different levels of ion exposure, particularly to understand how the surface of a REN film will be affected for use in devices where the surface layers are increasingly important.

Finally, we showed that $\mathrm{Au}$ and $\mathrm{Al}$ form ohmic contacts to $\mathrm{SmN}$ and $\mathrm{GdN}$ in vertical transport measurements when the $\mathrm{Au}$ is used as a bottom contact and the $\mathrm{Al}$ as a top contact, showing that they are suitable contact metals. Previous measurements in Ref [73] show that Gd and Nb can also successfully be used as contacts to GdN. 


\section{Chapter 6}

\section{Magnetic tunnel junctions}

A magnetic tunnel junction (MTJ) consists of two uncoupled ferromagnets (FMs), the electrodes. To uncoupled them they are separated by a thin insulator, the tunnel barrier. The MTJ electrodes are commonly made with transition metal FMs or dilute magnetic semiconductors (DMSs) [74]. The RENs offer an advantage over these as they allow independent control over the electrical and magnetic properties, allowing us to tune the REN properties to the intended application [1].

Depending on the application where the MTJ is going to be used, one can switch the magnetisation of the electrodes using a magnetic field, or using spin-transfer torque where a large spin-polarised current is used to switch the magnetisation of the electrodes selectively [74].

One common application of MTJs is in making magnetic random access memory (MRAM) where information is stored in the relative magnetic orientation of the two ferromagnets, called the free layer and the reference layer. Information is encoded by changing the magnetic orientation of one of the layers, the free layer, while the reference layer does not change. Information can be read out in multiple ways, but using the change in the tunnelling current at a set bias is an effective and noninvasive way of reading the state of the MRAM. This chapter presents a step towards making REN MRAM in the future. 
In this chapter, we present the development and manufacturing of GdN/GaN/SmN MTJs as well as characterisation and measurements of these devices. This chapter starts with a background on tunnelling and magnetic tunnel junctions. We then move on to experimental results, starting with structural characterisation of devices and films. We present measurements of the magnetisation of the devices. Then the electrical characteristics of a number of devices are presented, including I-V characteristics of devices within a single growth of 20 devices and how the I-V characteristics scale with device size. The electrical characteristics also include a discussion about the temperature and current dependent resistance, and an attempt to fit the J-V data using the Simmons model. The next section presents the junction magnetoresistance (JMR) of the devices which includes how the JMR changes with temperature, applied field, current, and device composition. We show switching of the devices when both the $\mathrm{GdN}$ and SmN are ferromagnetic. Lastly, among the results, we present the breakdown of devices and a comparison between the behaviour of working and broken devices. Finally, we discuss the behaviours observed and what this means for the tunnelling and magnetic alignment in our devices.

\subsection{Background}

Tunnel magnetoresistance (TMR) can be measured when a thin insulating barrier is held between two ferromagnets (FMs), and a voltage is applied. The current through the device is dominated by tunnelling of electrons through the barrier. Depending on the relative magnetic orientation of the two FMs, the tunnel current through the structure will be different. The magnitude of the tunnel current can be probed by measuring the device resistance.

Using the resistance as a way to probe the magnetic alignment essentially means measuring the resistance in parallel FMs (low resistance) and 
anti-parallel FMs (high resistance). Since FMs are spin split there are two spin currents moving through the MTJ - spin up (majority) and spin down (minority) currents, as illustrated in fig. 6.1. In the parallel state, the tunnelling is dominated by majority-majority band transport with a small contribution from minority-minority tunnelling. The majority-majority tunnelling is large as this is current from a large number of occupied states into a large number of unoccupied states. In contrast, in the anti-parallel state, the two tunnel currents are both small. Under the same applied bias, the majority-majority tunnel current in the parallel state is much larger than any of the other tunnel currents giving the parallel state a lower resistance than the anti-parallel state. Note that this is only true if the spin is conserved during the tunnelling process [74].

From this description the tunnel magnetoresistance is defined as the ratio of the resistance difference in the parallel and anti-parallel state to the parallel state

$$
T M R=\frac{R_{a p}-R_{p}}{R_{p}}
$$

The magnitude of tunnel currents, and consequently the TMR, will depend, among many things, on the density of states of the FMs at the Fermi level and the degree of spin polarisation of the FMs. The tunnelling probability is independent of temperature, but the density of states at the Fermi level in our materials is strongly temperature dependent. At lower temperatures, there are fewer thermally excited carriers which give higher spin polarisation which means we would expect a lower temperature to have a larger resistance difference between the parallel state and antiparallel states.

Tunnel magnetoresistance was discovered in 1975 by M Julièrre in $\mathrm{Fe} / \mathrm{Ge} / \mathrm{Co}$ junctions [75]. The initial discovery did not garner much attention from the community as the relative resistance change when changing the magnetic orientation was only $14 \%$ at $4.2 \mathrm{~K}$. In the $1990 \mathrm{~s} \mathrm{Fe} / \mathrm{Al}_{2} \mathrm{O}_{3} / \mathrm{Fe}$ devices with a larger TMR of $18 \%$ at room temperature and $30 \%$ at $4.2 \mathrm{~K}$ 


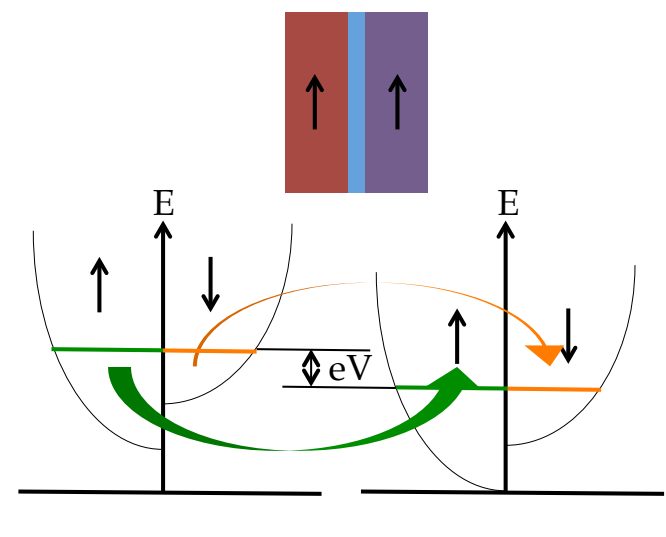

DOS FM1

(a)

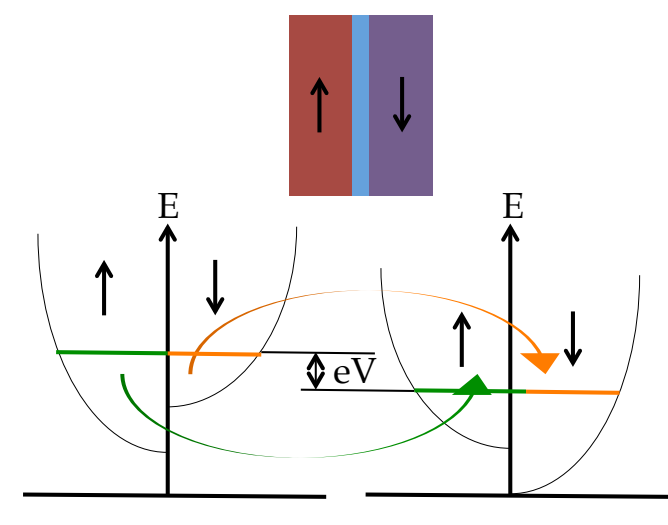

DOS FM1
DOS FM2

(b)

Figure 6.1: Spin polarised tunnel currents between the two ferromagnets with voltage difference $\mathrm{V}$ in (a) parallel magnetic orientation (b) anti-parallel magnetic orientation. In the parallel orientation, the large majority-majority tunnelling arises from a large number of filled states (green) in the first electrode which can tunnel to a large number of empty states (orange) in the second electrode. The other three tunnel currents are smaller as the electrons move between small differences in available density of states.

were constructed [76]. In 2008, with the discovery of $\mathrm{MgO}$ barriers, reported TMR in MTJs with MgO barriers increased to $220 \%$ [77] and then to $600 \%$ at room temperature [78].

Direct (elastic) tunnelling as described in fig. 6.1 does not predict a strong dependence on temperature. A strong temperature dependence can arise from a variety of effects such as pinholes, multi-step tunnelling via defects in the barrier, spin wave (magnon) or phonon-assisted tunnelling, interface scattering, and changes in the band structure [79-96]. The total tunnel current through the MTJ can be a combination of a number of tunnel current contributions, where different contributions may dominate at different temperatures and voltages. 
For a polycrystalline MTJ, particularly with a polycrystalline barrier, the transition between anti-parallel and parallel orientation is smeared out, rather than sharp, as the different crystallites will be slightly differently magnetically oriented [88].

A number of tunnel junction devices using the RENs have been demonstrated, for example: GdN spin filter devices [7,97], hybrid GdN/topological insulator devices [98], GdN/I/GdN MTJs [9, 73, 99], and GdN/Al$\mathrm{N} / \mathrm{SmN}$ MTJs $[9,99]$.

Figure 6.2, as reproduced from Ref [9], shows the magnetoresistance at $4 \mathrm{~K}$ of a GdN/GaN/GdN MTJ. In fig. $6.2 \mathrm{~b}$ ) the device displays a smooth resistance decrease with increasing field with a magnitude of $\sim 60 \%$ from $0 \mathrm{~T}$ to $8 \mathrm{~T}$. The TMR of the GdN/GaN/GdN junctions in Ref [9] display a local TMR maximum at $50 \mathrm{~K}$ and a global TMR maximum at $2 \mathrm{~K}$. The MTJ in fig. 6.2 shows a smooth resistance decrease with increasing field. Figure 6.2 a) shows a low field magnification of fig. 6.2 b), where the switching of one of the GdN layers is clearly visible.

In a GdN/I/SmN MTJ the expected behaviour is different from standard MTJs. As GdN is a spin-dominated FM and SmN is an orbital dominated FM we expect the two electrodes to align with the total spin antiparallel to each other in an applied magnetic field. This means that the electrodes should be parallel at low field, and anti-parallel at high fields.

The unconventional TMR behaviour is shown in fig. 6.3, as reproduced from Ref [99], where the GdN/AIN/SmN MTJ show a positive TMR as expected for electrodes of different spin alignment. The dip between $1 \mathrm{~T}$ to $4 \mathrm{~T}$ was attributed to the magnetisation of the SmN layer as this was of the order of the $\mathrm{SmN}$ coercive field and showed no hysteresis, while the complicated low field behaviour was attributed mainly to the changes in the magnetisation of the $\mathrm{GdN}$ layer. 
a)

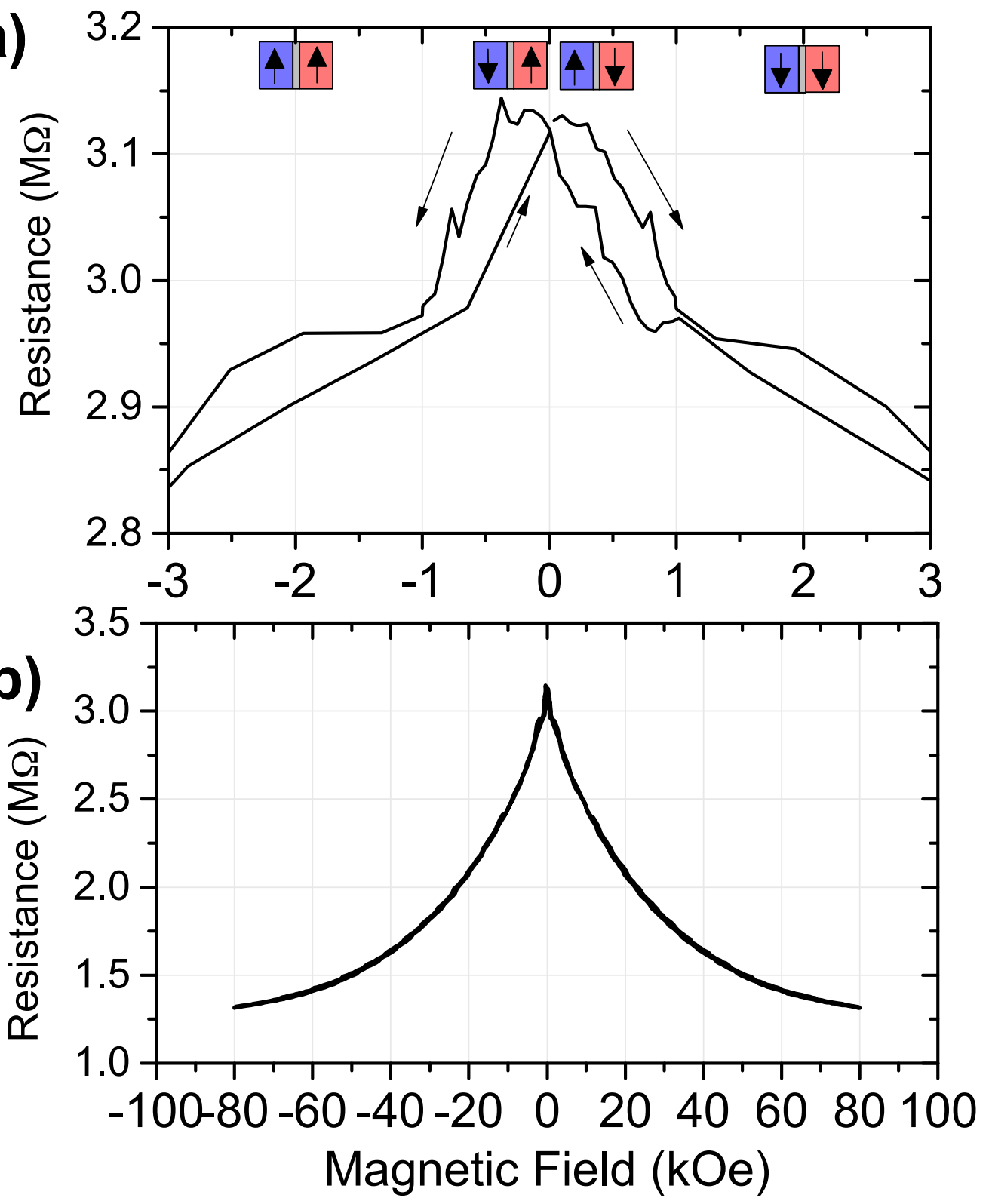

Figure 6.2: GdN/GaN/GdN MTJ measured at $50 \mathrm{nA}$ at $4 \mathrm{~K}$ with field inplane. Panel a) is a magnification of the low-field behaviour from panel b) displaying the switching of on of the GdN electrodes. Reproduced from Ref [99] with permission. 


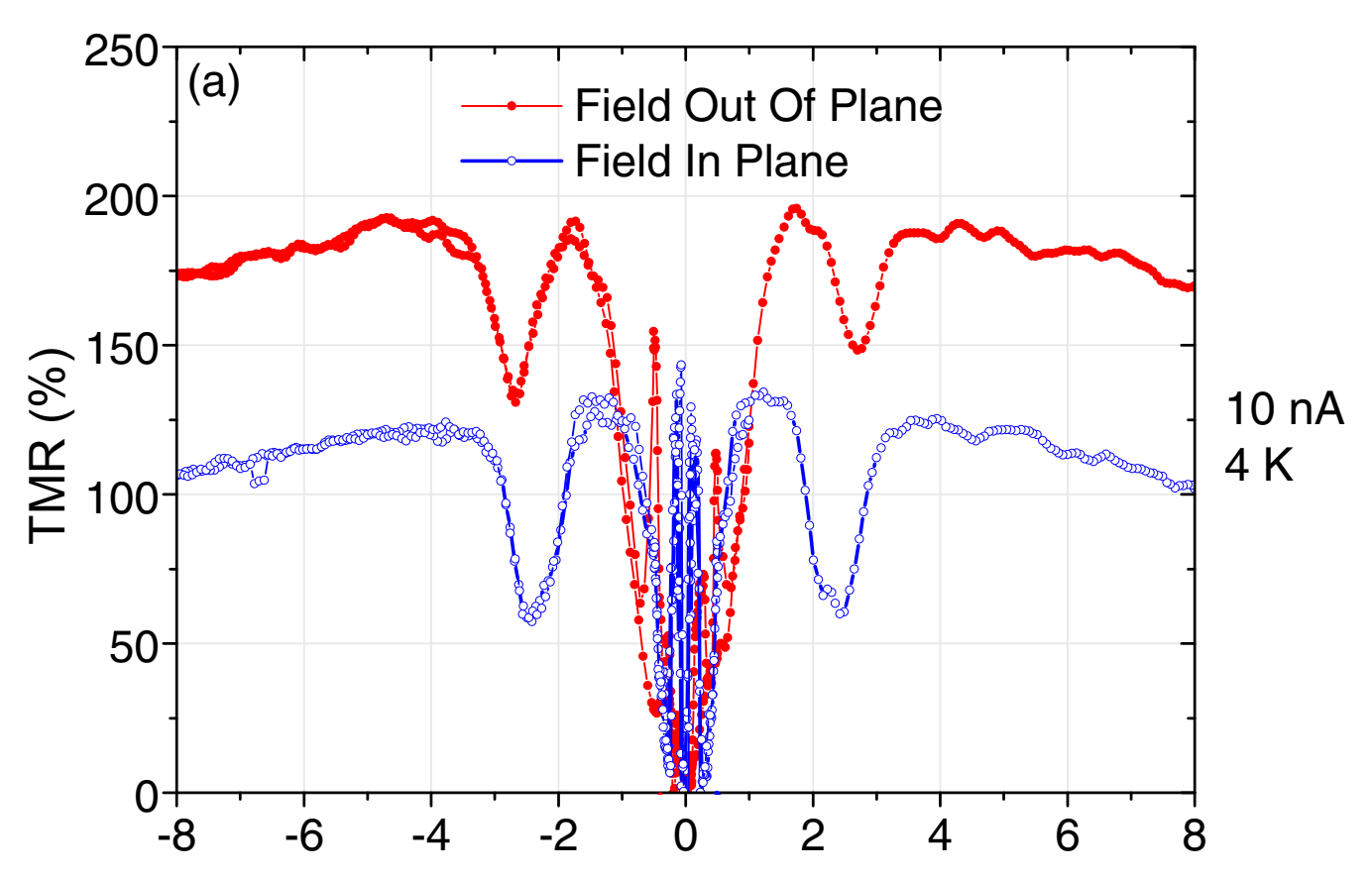

Field (T)

Figure 6.3: GdN/AIN/SmN MTJ measured at $10 \mathrm{nA}$ at $4 \mathrm{~K}$. The TMR shows contributions from the magnetisation changing in both $\mathrm{GdN}$ and SmN at different applied fields. Reproduced from Ref [9] with permission. 


\subsection{GdN/GaN/SmN MTJs}

The data shown in this chapter is mainly from two different sample manufacturing growths. Each growth yields 20 devices with 15 different active areas, making it possible to study device consistency, device substrate placement, as well as any effects the in-plane device dimensions might produce. More details on the manufacturing process can be found in section 3.5.

In the $\mathrm{GdN} / \mathrm{GaN} / \mathrm{SmN}$ devices, the $\mathrm{GdN}$ is always used as the bottom electrode due to its smoother growth in the polycrystalline phase as mentioned in chapter 2 . The $\mathrm{N}_{2}$ pressure is kept constant in the growth chamber to minimise changes to the surface composition of the RENs from a fluctuating $\mathrm{N}_{2}$ pressure while preparing sources for each subsequent layer in the MTJ.

The first MTJ sample is a highly resistive sample, here on referred to as HR. HR has a bottom GdN electrode, a GaN barrier (designed thickness: $3 \mathrm{~nm}$ ), a top SmN electrode, and a Gd metallic top contact also acting as a capping layer. Sample HR is named A516 in lab books.

The second MTJ sample is a less resistive sample, here on referred to as LR. LR has a bottom GdN electrode, a thinner GaN barrier (designed thickness: $2 \mathrm{~nm}$ ), a top SmN electrode, and an Al metallic top contact also acting as a capping layer. Sample LR is named A540 in lab books.

The differences between the devices are the top contact material and the thickness of the barrier. The resistance difference between the two samples is dominated by the difference in the thickness of the barrier. The two top contact metals were chosen to study potential effects from using a ferromagnetic Gd contact as well as a non-magnetic Al contact.

Both samples have an Au bottom contact deposited on a highly resistive $\left(\rho_{S i}>1000 \Omega \mathrm{cm}\right)$ Si substrate. Both samples also have a $35 \mathrm{~nm}$ thick $\mathrm{GaN}$ area restriction layer which defines the active areas of the devices on the substrates. 
A diagram of the layers making up each device can be seen in fig. 6.4. The area restriction layer is not shown in the diagrams, as the diagram is intended to depict the layers inside the active area.

High resistance

(HR)

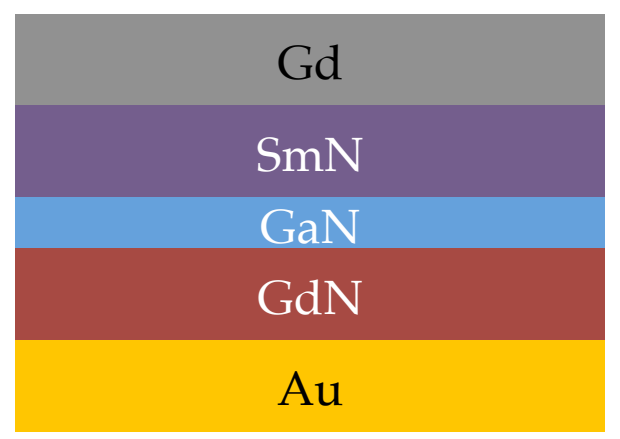

Low resistance

(LR)

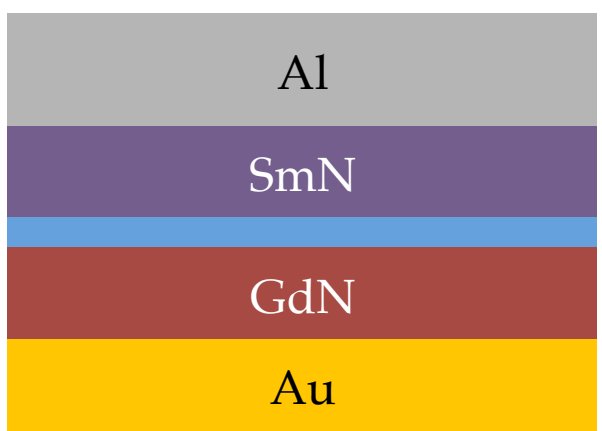

Figure 6.4: Layers of the two samples presented in this chapter. The samples only differ by the prepared GaN tunnel barrier thickness and the top contact material; otherwise, the two growths are nominally the same. Dimensions are not to scale.

When data is referred to as from a "Sample" (for example XRD and SEM), the data is taken as being representative of the entire sample, whereas when data is referred to as from a "Device", then the data is from an individual device from a particular sample growth. For example "HR $20 \mu \mathrm{m}$ " is a device from Sample growth HR with an active area of $20 \mu \mathrm{m} \times 20 \mu \mathrm{m}$.

Figure 6.5 show a schematic of the device. The layer thicknesses are greatly exaggerated compared to the in-plane dimensions for clarity. The outer dimensions of the bottom gold electrode are $8 \mathrm{~mm} \times 9 \mathrm{~mm}$ while the REN layer thicknesses are on the order of $50 \mathrm{~nm}$. More detailed discussion on the pattern can be found in section 3.5. 


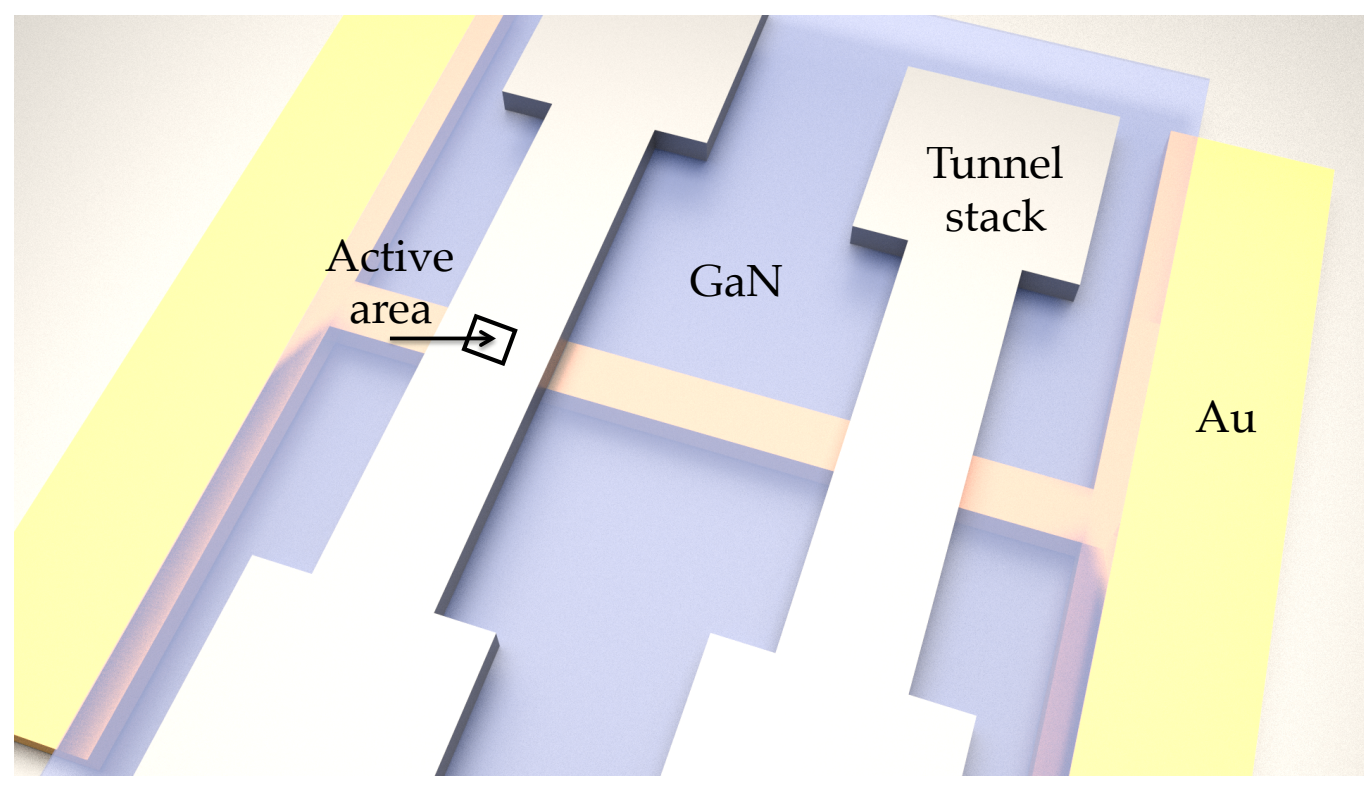

Figure 6.5: The device structure of a single chip showing the common $\mathrm{Au}$ bottom contact for two devices and the GaN area restriction layer. The two devices on each chip have different active areas. Each sample growth makes 10 chips and hence 20 devices. The arrow on the left device marks where in the device the active area is located.

\subsubsection{Structural characterisation}

This section shows initial structural characterisations of sample HR and sample LR. The samples were investigated using an optical microscope, SEM, and XRD. The measurements conducted include pattern conformity, layer thickness and pattern quality as well as crystal structure distributions.

All devices are patterned using photoresist which necessitates that the samples are grown at room temperature yielding polycrystalline layers. The devices are patterned using photolithography in many steps. Multistep photolithography runs the risk of residual photoresist getting trapped on rough surfaces left behind from previously patterned growths. In fig. 6.6 (a) the centre of a proto-device is imaged using an optical microscope with 
10x magnification after the final photoresist is added but before the REN stack is deposited to make the finished device. The yellow bar horizontally across the image is the Au bottom contact. The GaN area restriction layer covers the entire image in fig. 6.6 (a), except for the active area square in the middle of the image where direct contact will be made between the MTJ stack and the bottom Au contact. The corners of the active area are slightly rounded, as the pattern resolution achieved for the devices is limited to $5 \mu \mathrm{m}$. The vertical line on the left side of the image has photoresist to the left of it, and GaN and the active area to the right which is not covered in the photoresist.

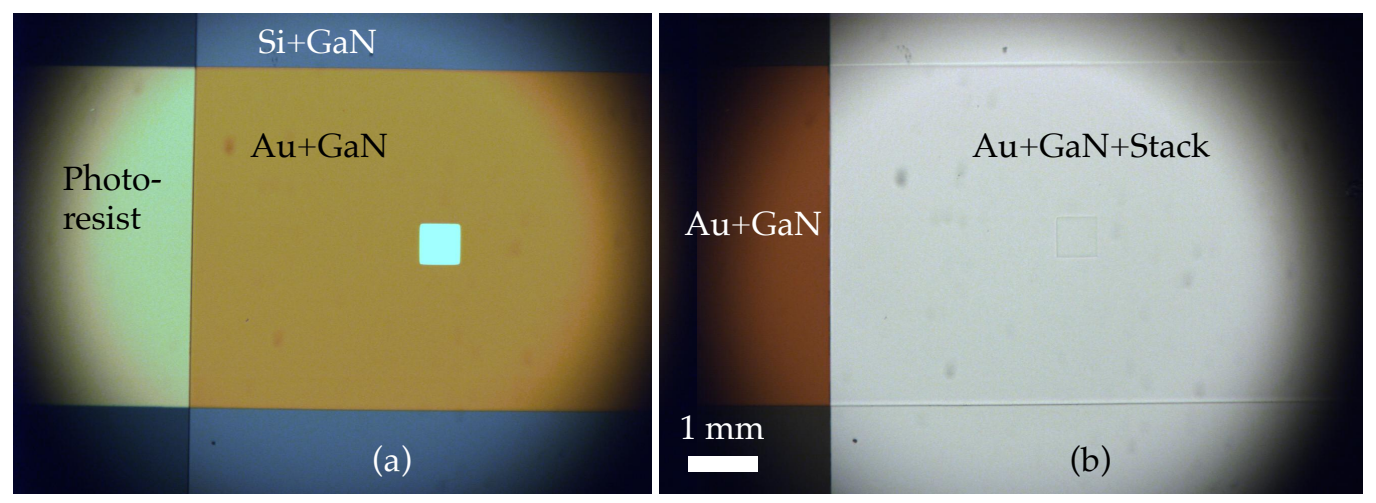

Figure 6.6: Optical microscope image of the junction of a (a) proto device before the stack deposition showing junction shape and (b) device after stack deposition showing a very faint outline of the active area. The faint black smudges are artefacts from dirt inside the microscope, present in all images.

Figure 6.6 (b) shows the same device as fig. 6.6 (a) but after the REN tunnel stack has been deposited. There is still a very faint outline of the cut-out in the GaN layer, which is the active area of the device. The REN tunnel stack covers the $\mathrm{Au}$ and GaN smoothly with no apparent defects present.

In fig. 6.7 a secondary electron image (SEI) has been taken of the junction active area of a LR device. The edges of the active area show some 
intensity variation. While studying the inset in fig. 6.7, which show a closeup of the marked area, the intensity variation arises from the edge protruding over the rest of the layer.

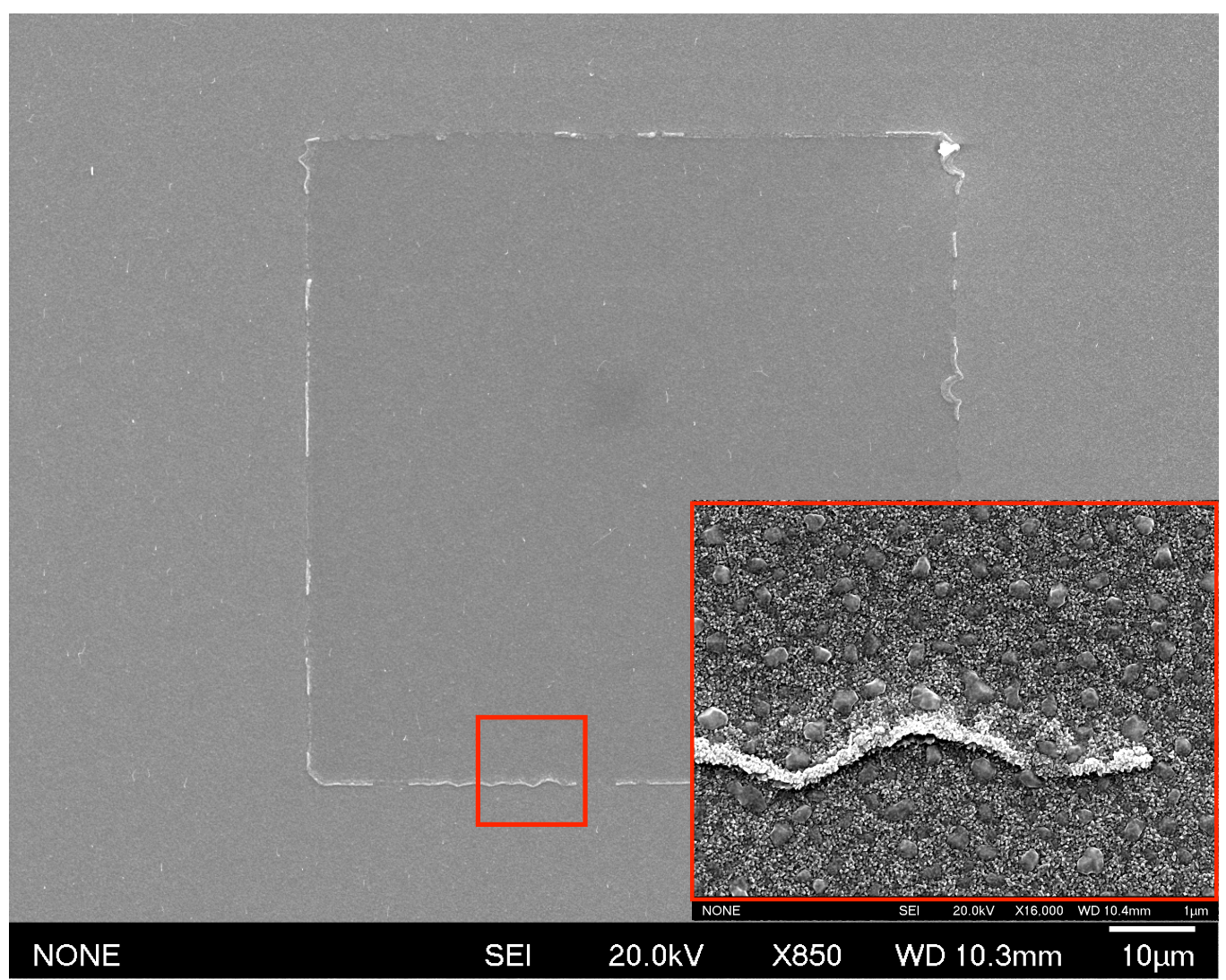

Figure 6.7: SEM image of the active area of a finished device. The inset shows a magnification of the area in the red square. The surface is the $\mathrm{Al}$ top contact and the rounded shapes in the inset are likely Al metal droplets.

Figure 6.8 show sample HR measured in three measurement modes of the SEM. In panel (a) the EDS map shows the prevalence of certain elements in the different layers of the sample. It is clear that in the HR sample we have two Gd-containing layers (green), one layer containing Sm (red), and a bottom layer containing Ga (blue). The tunnel barrier is too thin to 
be imaged at the resolution of EDS in this SEM. Panel (b) shows the same image but measured in SEI mode. The SEI image clearly shows the structure and thickness of the different layers; even the GaN barrier is visible as a thin line. In panel (c) the same image is shown in backscatter mode which clearly shows the different elements as the intensity is correlated with elemental mass.

The XRD spectra are all taken on the large unpatterned areas of the substrate wafer. The spectra between $29^{\circ}$ to $34^{\circ}$ for samples HR and LR can be seen in fig. 6.9. Both samples show characteristic peaks for GdN, $\mathrm{SmN}, \mathrm{Gd}, \mathrm{Al}$, and $\mathrm{Si}$ (not all shown in this figure). The spectra have been aligned to the $\mathrm{Si}(400)$ peak at $69.17^{\circ}$.

As is often the case in polycrystalline $\mathrm{GdN}$ and $\mathrm{SmN}$ the (111) orientation is the dominant peak, located at $31.2^{\circ}$ and $30.7^{\circ}$ respectively. The relative strength of the $\mathrm{SmN}(111)$ and $\mathrm{GdN}(111)$ peaks is not the same for the two samples. As well as being relatively weaker, the $\mathrm{SmN}(111)$ in sample HR appears broadened compared to the $\mathrm{SmN}(111)$ peak in sample LR. As was discussed in section 5.3.2 it is possible that the there is an expanded GdN(111) phase at $30.7^{\circ}$ which coincides with the $\mathrm{SmN}$ (111) peak, making it impossible to reliably identify from these XRD spectra. There is also a possible $\mathrm{Gd}(002)$ peak in sample $\mathrm{HR}$ at $30.7^{\circ}$ which may contribute to the appearance of a widened $\mathrm{SmN}(111)$ peak. 
(a)
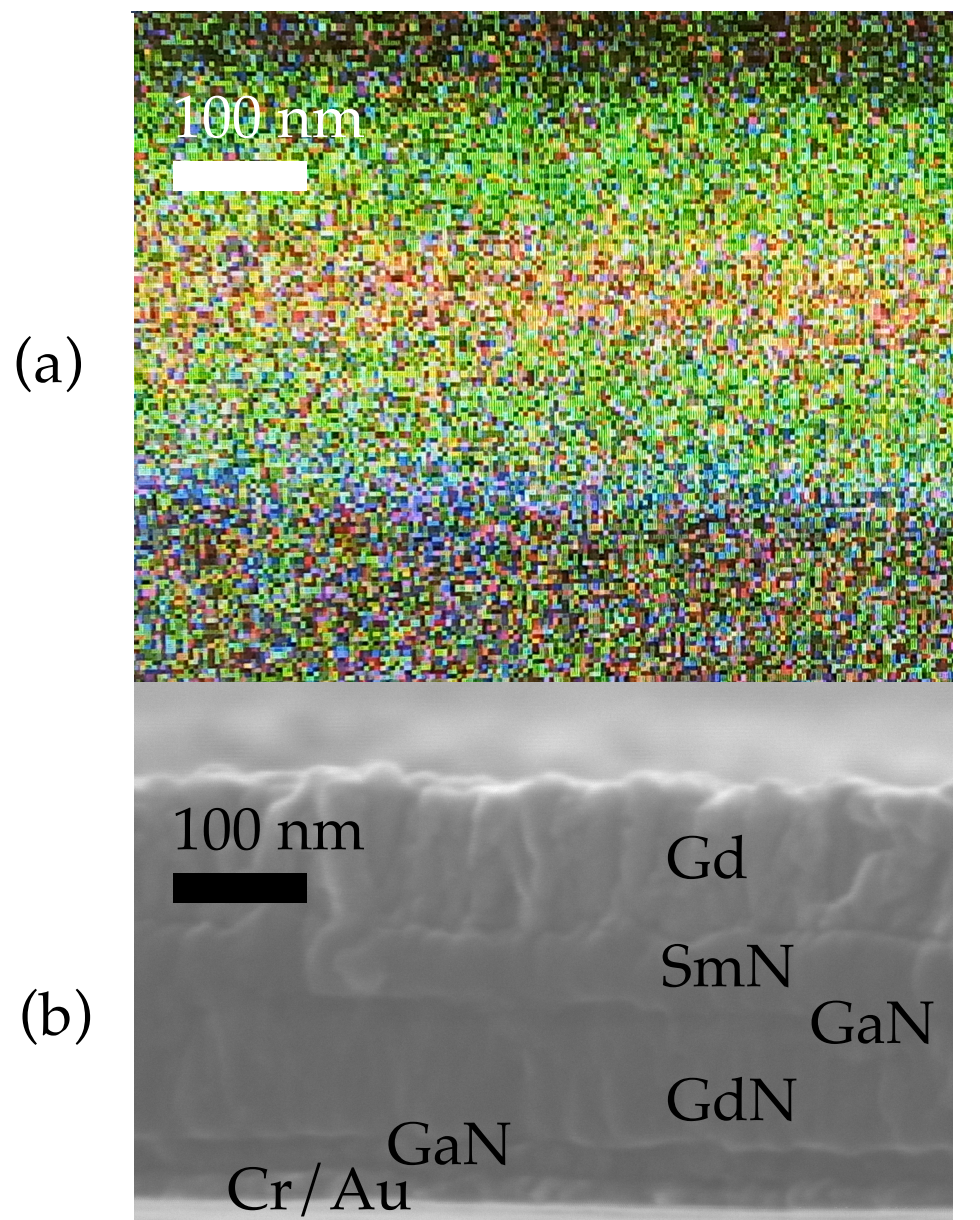

(c)

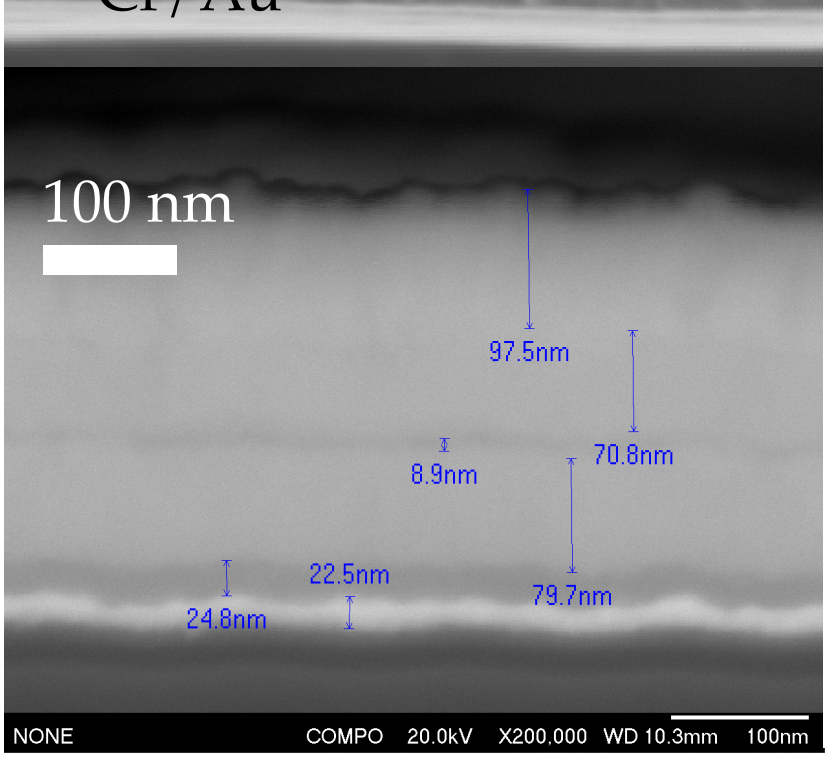

Figure 6.8: Cross-sectional SEM images of Sample HR: (a) EDS map (Green: Gd, Red: Sm, Blue: Ga) (b) SEI, (c) BSI. The layers are well defined with thicknesses close to the designed thicknesses. Note that the thickness of the tunnel barrier as indicated in (c) is merely the smallest number that can be assigned to a layer in the software. The scale is the same in all three images and indicated by the scale bar. 


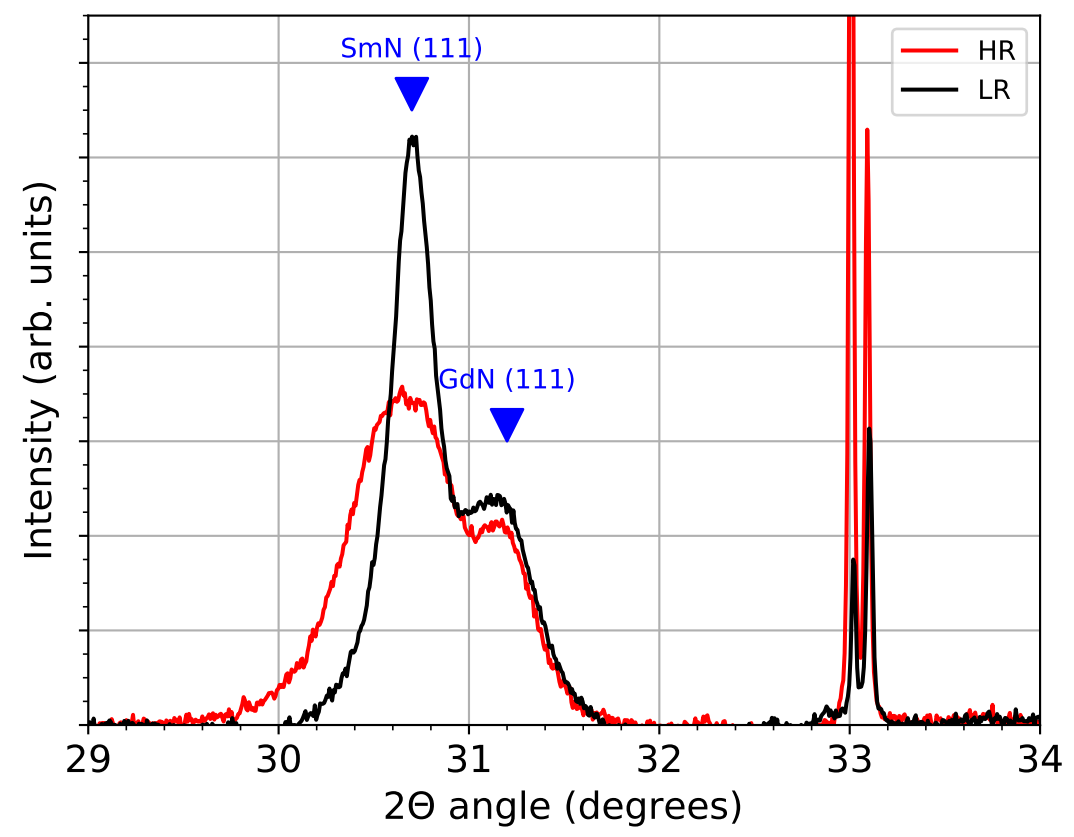

Figure 6.9: XRD of sample HR and LR, zoomed in around the $\mathrm{SmN}(111)$ and $\mathrm{GdN}(111)$ peaks. The $\mathrm{SmN}(111)$ peak in sample HR is potentially widened by a $\mathrm{Gd}(002)$ peak at $30.7^{\circ}$. 


\subsubsection{Magnetic moment}

To compare the sample magnetisation with subsequent magnetoresistance measurements, we present temperature- and field dependent magnetisation curves for samples HR and LR. These measurements were conducted on an unpatterned piece of the sample, meaning it contains all the same layers as the device, but the layers have an in-plane area of around $3 \mathrm{~mm} \times 8 \mathrm{~mm}$. Due to the magnitude differences in the magnetic moment of $\operatorname{GdN}\left(7 \mu_{\mathrm{B}} / \mathrm{Gd}^{3+}\right.$ ion) [29] and $\mathrm{SmN}\left(0.0035 \mu_{\mathrm{B}} / \mathrm{Sm}^{3+}\right.$ ion) [24] we are likely to only see $\mathrm{GdN}$ in the measurements.

The temperature dependent magnetisation for sample HR and LR are shown in fig. 6.10 and fig. 6.11, respectively. Figure 6.10 shows a field cooled measurement in $0.02 \mathrm{~T}$ of sample HR, and fig. 6.11 shows both field cooled and and zero-field cooled measurements in $0.02 \mathrm{~T}$ of sample LR.

Sample HR, in fig. 6.10, shows a very gradual onset of the magnetisation. This is influenced by the Gd top contact layer, as Gd has a Curie temperature of $293 \mathrm{~K}$ with a small temperature dependency [100]. A fit to the paramagnetic phase returns $\mathrm{T}_{C}=62 \mathrm{~K}$, corresponding well to measurements of GdN in chapter 5 and literature [29].

Sample LR, in fig. 6.11, shows a more sudden onset in the magnetisation. A fit to the paramagnetic phase returns $\mathrm{T}_{C}=70 \mathrm{~K}$, also corresponding well to measurements in literature [1]. The zero-field-cooled curve in fig. 6.11 has a feature centred around $55 \mathrm{~K}$, demonstrating a double ferromagnetic transition. The double transition has been identified previously, particularly in epitaxial GdN samples, as well as in the ion exposed GdN in section 5.3.2 [29]. The double transition may be present in the HR sample as well, but the contribution from the Gd layer obscures the measurement. The larger magnitude of the magnetic moment in fig. 6.11, as compared to fig. 6.10, is due to the much sharper hysteresis loop in sample LR, where at a low field the magnetisation is a more than an order of magnitude higher than in sample HR. 


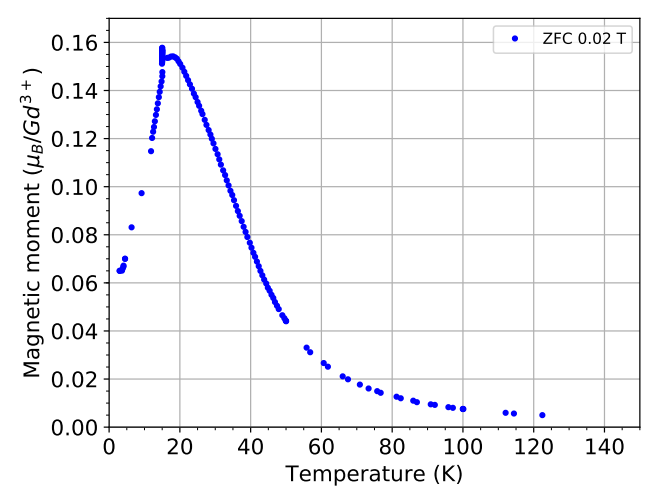

Figure 6.10: HR: Temperature dependent magnetisation of sample $\mathrm{HR}$ with $\mathrm{ZFC}$ in $0.02 \mathrm{~T}$.

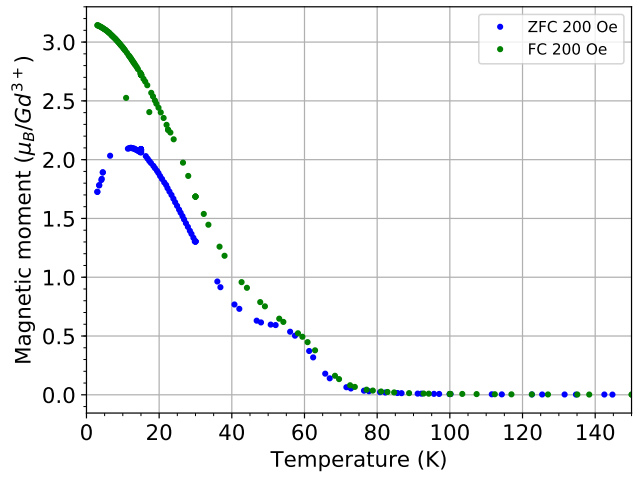

Figure 6.11: LR: Temperature dependent magnetisation of sample LR with ZFC and FC in $0.02 \mathrm{~T}$.

The magnetic moment as a function of applied in-plane field of sample LR and HR are plotted in fig. 6.12 and fig. 6.14, with low field magnifications in fig. 6.13 and fig. 6.15, respectively. Both samples have a small coercive field, but the high field behaviour is different.

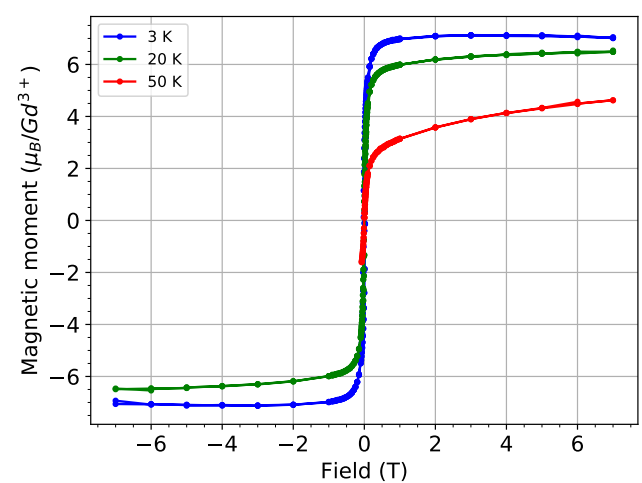

Figure 6.12: LR: Magnetic hysteresis loop of sample LR at $3 \mathrm{~K}, 20 \mathrm{~K}$, and $50 \mathrm{~K}$.

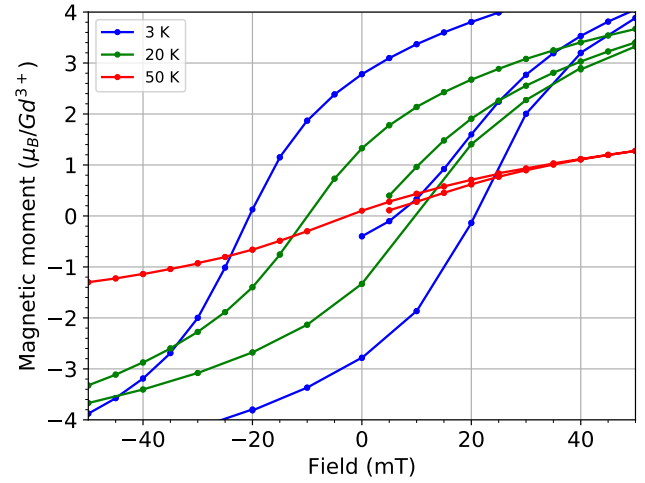

Figure 6.13: LR: Low field magnification of fig. 6.12 displaying the magnitude of the coercive field in sample LR. 


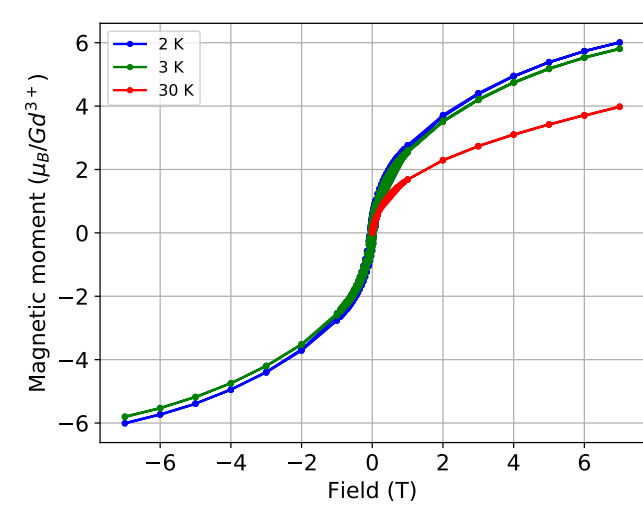

Figure 6.14: HR: Magnetic hysteresis loop of sample HR at $2 \mathrm{~K}, 3 \mathrm{~K}$, and $30 \mathrm{~K}$.

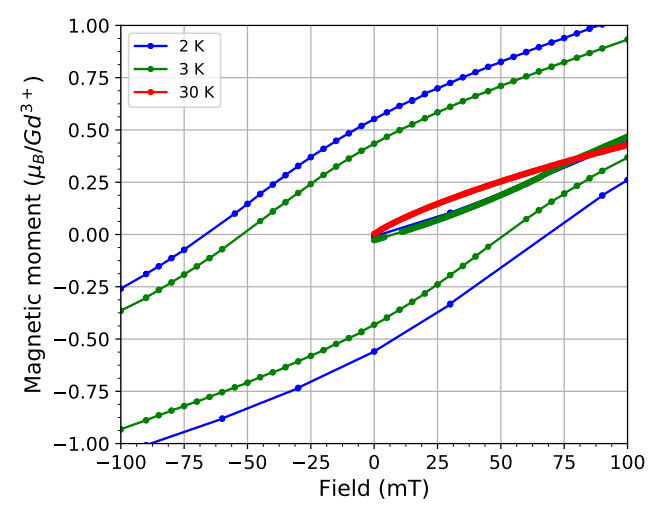

Figure 6.15: HR: Low field magnification of fig. 6.14 displaying the magnitude of the coercive field in sample HR.

Sample LR in fig. 6.12 displays a typical polycrystalline GdN hysteresis loop with a comparatively well saturated magnetic moment from around $1 \mathrm{~T}$ at $3 \mathrm{~K}$. The moment saturates with a fairly square shape at $3 \mathrm{~K}$, and more gradually at $20 \mathrm{~K}$. At $50 \mathrm{~K}$ the moment is not saturating even at $7 \mathrm{~T}$. The coercive field can be seen in fig. 6.13 and is $0.02 \mathrm{~T}$ at $3 \mathrm{~K}$ and drops for higher temperatures, which is consistent with previous measurements of GdN [21, 29,30,101].

In contrast, sample HR in fig. 6.14 does not appear to saturate the magnetic moment even at $2 \mathrm{~K}$. The difference in saturation field may arise from the GdN layer being changed by the ions during the tunnel barrier growth, as described in section 5.3. As the GdN layer in the HR devices has been exposed to ions longer than the GdN layer in the LR devices, for a longer tunnel barrier growth, it is possible that the GdN has been changed more in the HR sample. The higher saturation field could also suggest the presence of oxygen in the GdN or Gd top contact [102,103]. Since gadolinium oxides are paramagnetic or even antiferromagnetic [102] the overall magnetisation of the sample will be lower as there is less ferromagnetic Gd and GdN. If the lower saturation moment is due to oxygen, the obtained 
results could be achieved with an oxygen concentration of less than a few tenths percent [102].

Figure 6.15 show a low field magnification of the HR hysteresis loop, clearly displaying the coercive field. The coercive field for sample HR is $0.07 \mathrm{~T}$ at $3 \mathrm{~K}$. It is difficult to say if this enlarged coercive field (relative to sample LR) is due to the GdN itself, the Gd layer, the oxidised Gd top layers, or from the potentially oxidised $\mathrm{GdN}$ film. Gadolinium oxide in literature appears to have low coercive fields implying this is not the cause of the enlarged coercive field $[103,104]$.

\subsubsection{Tunnelling measurements at $0 \mathrm{~T}$}

Electrical measurements of devices have been done in the Janis research closed cycle cryostat as well as the Quantum design Physical Property Measurement System, see chapter 3 for details on both machines and measurements. All devices were measured with the current perpendicular to the plane of the film, and with the bottom Au contact set as the positive terminal.

All devices from all MTJ growths show non-linear current-voltage characteristics, in stark contrast to the electrical characteristics in simple GdN or SmN films in section 5.4 which have linear current-voltage characteristics.

We will see in this section that a device shows non-linear behaviour until it is exposed to high voltages. The application of a high voltage breaks the junction, and the resulting broken junction has linear J-V characteristics. The breakdown is assumed to occur in the tunnel barrier, lending credibility to the unbroken devices having a different conduction mechanism to pure GdN/SmN films where no tunnel barrier is present. 


\section{Temperature dependent resistance}

The temperature dependent resistance of a HR device and a LR device are shown in fig. 6.16 and fig. 6.17, respectively, for a number of different currents. At first glance, the curves appear to be very similar to the temperature dependent resistance of $\mathrm{GdN}$ and $\mathrm{SmN}$ films, but it is important to note that the resistance measured is orders of magnitude too large to arise from resistive transport through the $\mathrm{GdN}$ or $\mathrm{SmN}$ films. The resistance of similarly structured devices but without a tunnel barrier show a resistance of $\sim 1 \Omega$ establishing that the resistance of the MTJs is dominated by the tunnel barrier.

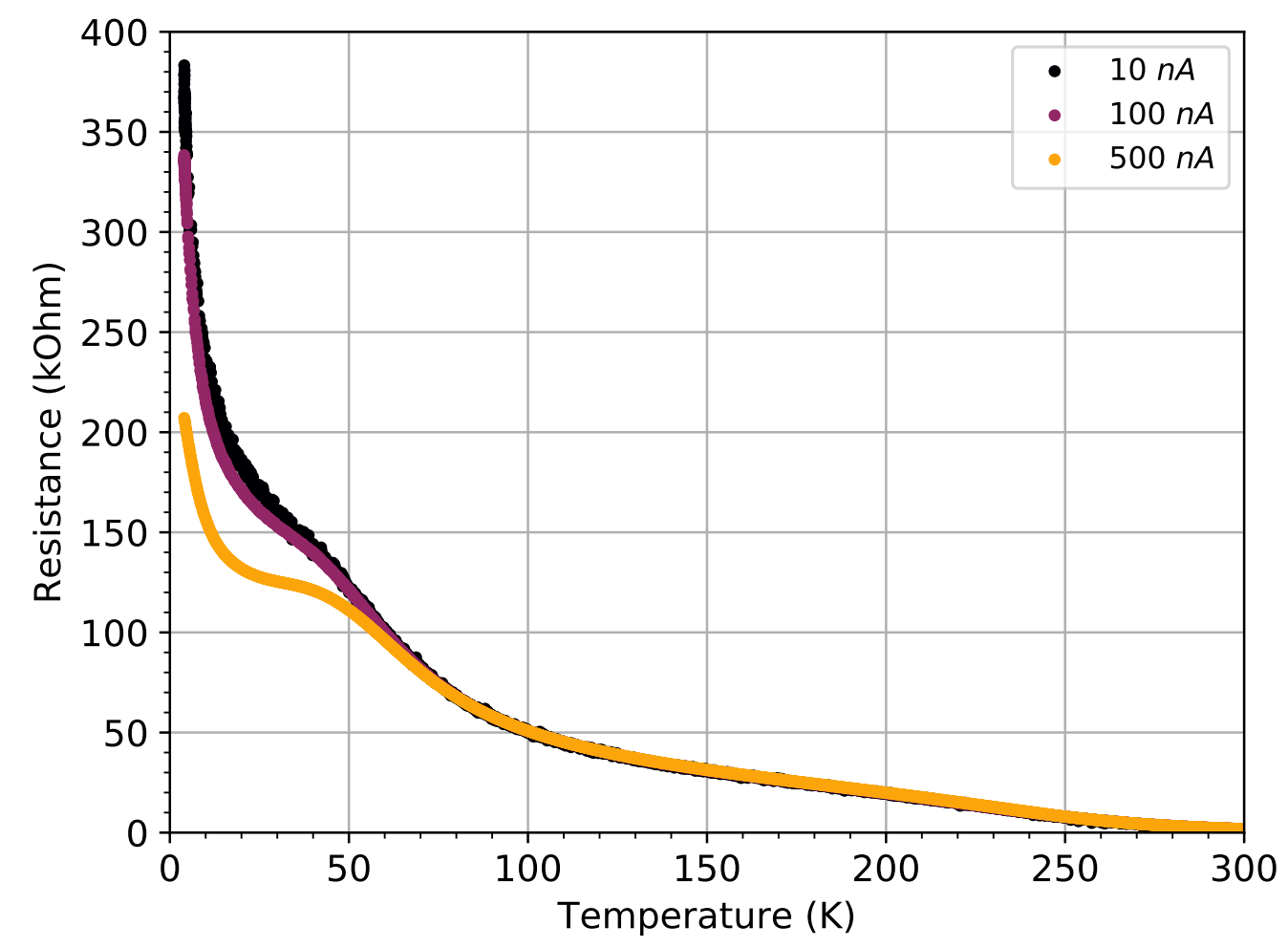

Figure 6.16: HR $(60 \mu \mathrm{m})$ : Resistance vs temperature measured with different currents.

Both fig. 6.16 and fig. 6.17 show a strong current dependence which is 
not seen in similar structures without a tunnel barrier or in in-plane transport in $\mathrm{GdN}$ or $\mathrm{SmN}$ films. The current dependence is stronger at lower temperatures. This suggests a study of the current-voltage characteristics should be the next step.

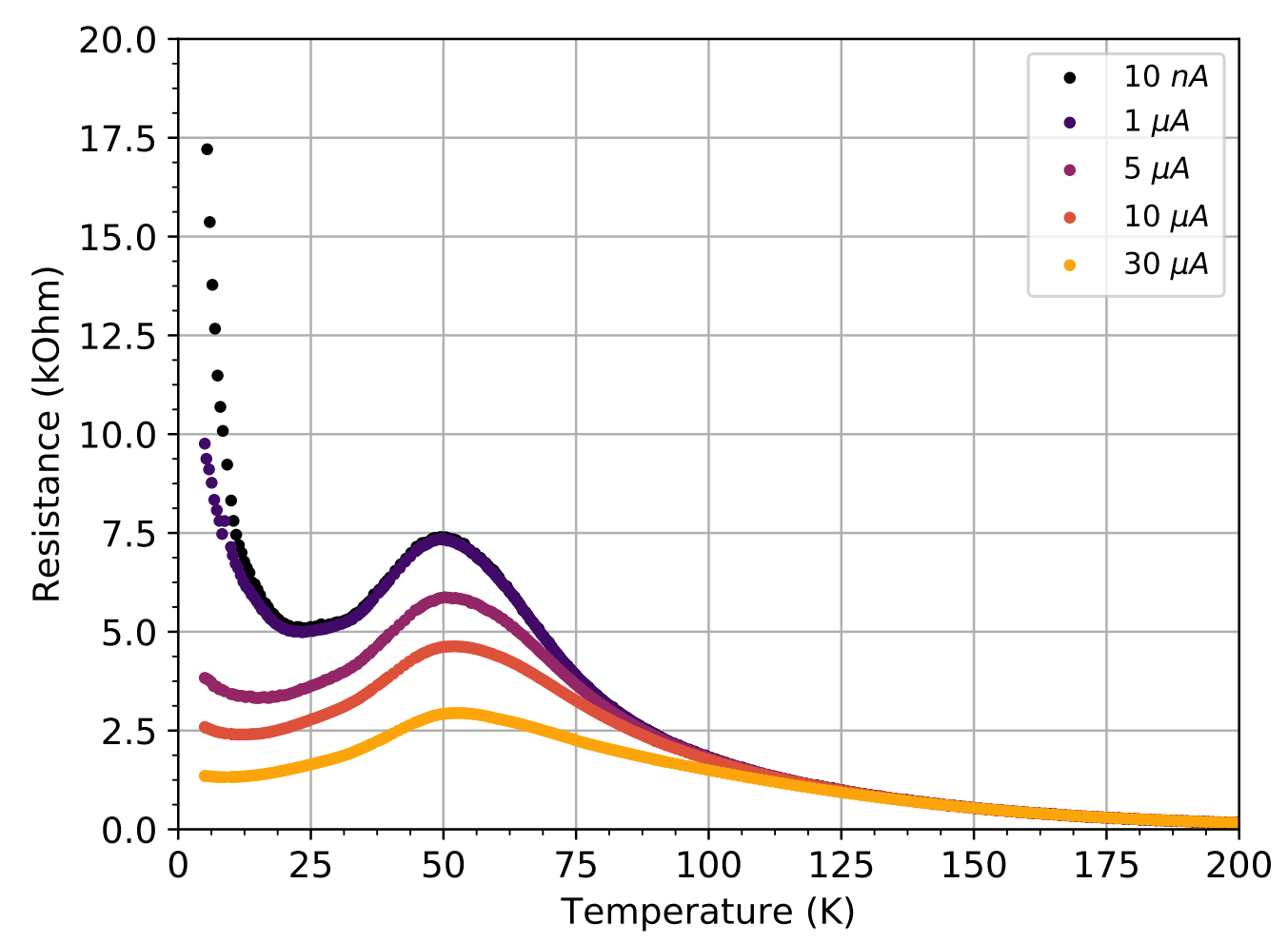

Figure 6.17: LR $(20 \mu \mathrm{m})$ : Resistance vs temperature measured with different currents.

In both fig. 6.16 and fig. 6.17 there is a large feature around $50 \mathrm{~K}$ in the resistance curves. In fig. 6.17 there is also a small feature around $30 \mathrm{~K}$, which appears as a flat region in the low current curves and as a small shoulder in the higher current curves. The $30 \mathrm{~K}$ feature likely exists in fig. 6.16 but is obscured by the large resistance increase. We note that these temperatures are close to $\mathrm{T}_{C}$ of the $\mathrm{GdN}$ and $\mathrm{SmN}$ electrodes; clearly demonstrating that the ferromagnetism of both electrodes influences the tunnelling resistance. Above $\mathrm{T}_{C}$ of the two electrodes, the model 
presented in fig. 6.1 is not spin split, but below $\mathrm{T}_{C}$ the band structure of the RENs changes, spin-splitting the bands and shifting the band edges $[105,106]$.

\section{Current density-voltage characteristics}

We now turn to detailed current density-voltage characteristics of representative devices. This section will present data primarily from the same two samples as described in fig. 6.4: High Resistance - HR and Low resistance - LR, but describe a number of devices from each sample.

Figure 6.18 shows the J-V characteristics between $\pm 1000 \mathrm{mV}$ of a HR device (active area $60 \mu \mathrm{m} \times 60 \mu \mathrm{m}$ ) at temperatures between $4 \mathrm{~K}$ to $300 \mathrm{~K}$. All temperature curves show non-linear $\mathrm{J}$ increase with high voltage but are linear at low voltages.

The HR devices show a moderate temperature dependence above $70 \mathrm{~K}$, with higher temperatures being more conductive in keeping with the curves in fig. 6.16. As the temperature goes below $30 \mathrm{~K}$ the device becomes more conductive again at high voltage, which is clearly seen in the high voltage magnification in the inset of fig. 6.18. The change in behaviour around $\mathrm{T}_{C}$ of $\mathrm{SmN}$ again indicates that a change in the tunnelling from a change in the band structure.

The J-V characteristics in fig. 6.18 are slightly asymmetric. As the device layers are asymmetric around the tunnel barrier ( $\mathrm{GdN}$ below, $\mathrm{SmN}$ above), and the density of states of the electrode will be different there is no reason to expect the J-V characteristics to be symmetric [107]. For simplicity, future figures in this chapter we will focus on the positive part of the curve, but the conclusions drawn are valid, unless otherwise stated, for both current directions. All devices are measured with the bottom contact of the devices as the positive terminal.

The positive part of the J-V characteristics in fig. 6.18 is shown on a logarithmic scale in fig. 6.19. Plotting the data on a logarithmic scale allows us to study the low voltage behaviour more easily. 


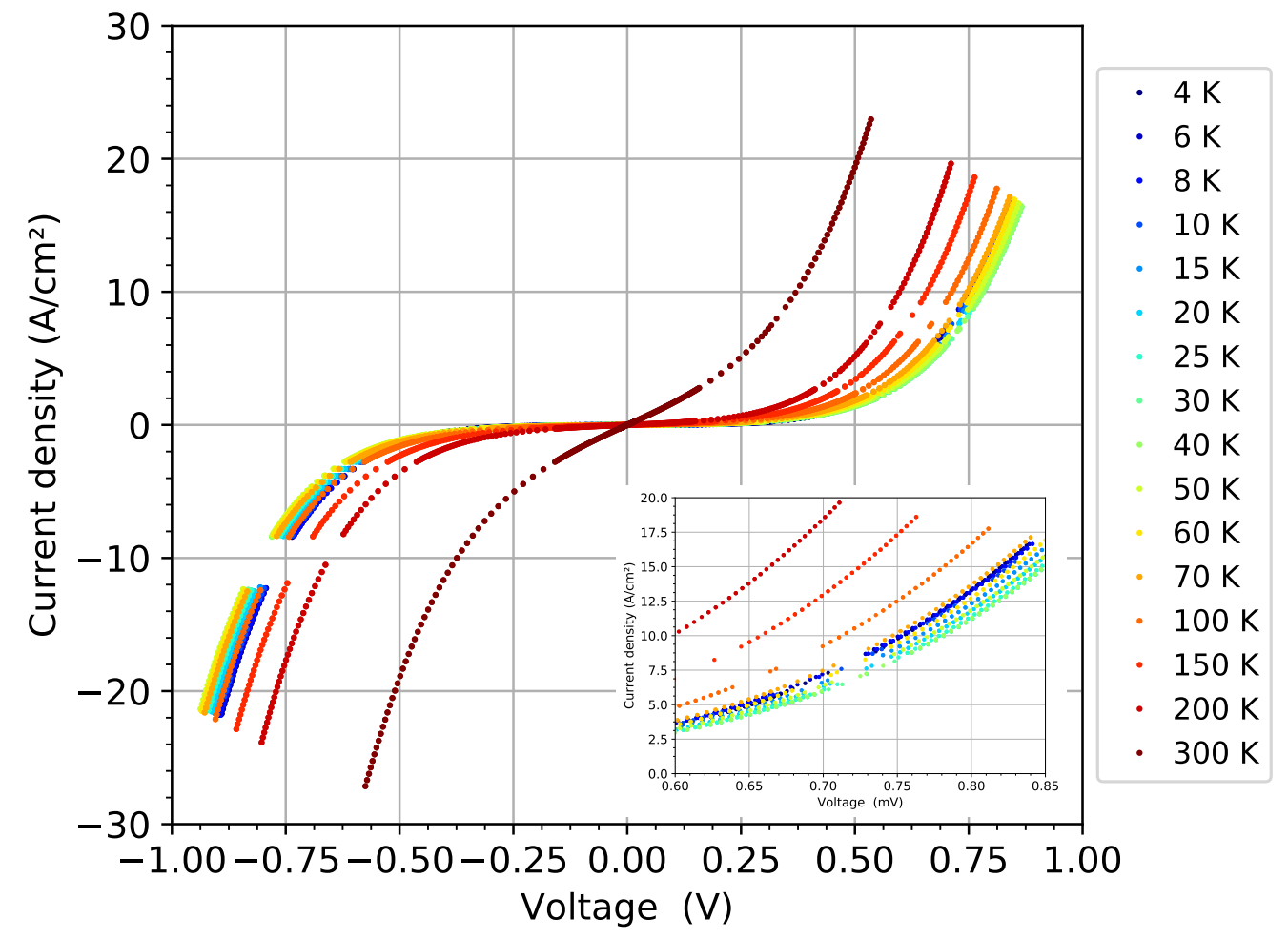

Figure 6.18: HR $(60 \mu \mathrm{m})$ : J-V curves at different temperatures between $\pm 1000 \mathrm{mV}$. Inset shows a magnification of the high voltage positive curves between $600 \mathrm{mV}$ to $850 \mathrm{mV}$ and $0 \mathrm{~A} / \mathrm{cm}^{2}$ to $20 \mathrm{~A} / \mathrm{cm}^{2}$.

The HR device in fig. 6.19 shows linear J-V characteristics at low voltages for all temperatures and non-linear behaviour at higher voltages. The curves become non-linear at lower voltages for lower temperatures. On the logarithmic scale, it is evident that the temperatures below $50 \mathrm{~K}$ cross over the higher temperatures around $300 \mathrm{mV}$. This is equivalent to a higher current curve than measured in fig. 6.16, as a cross over corresponds to a lower resistance for the crossed over data points. This is equivalent to the high current curves in the LR sample in fig. 6.17 where the resistance does not increase again below $50 \mathrm{~K}$. 


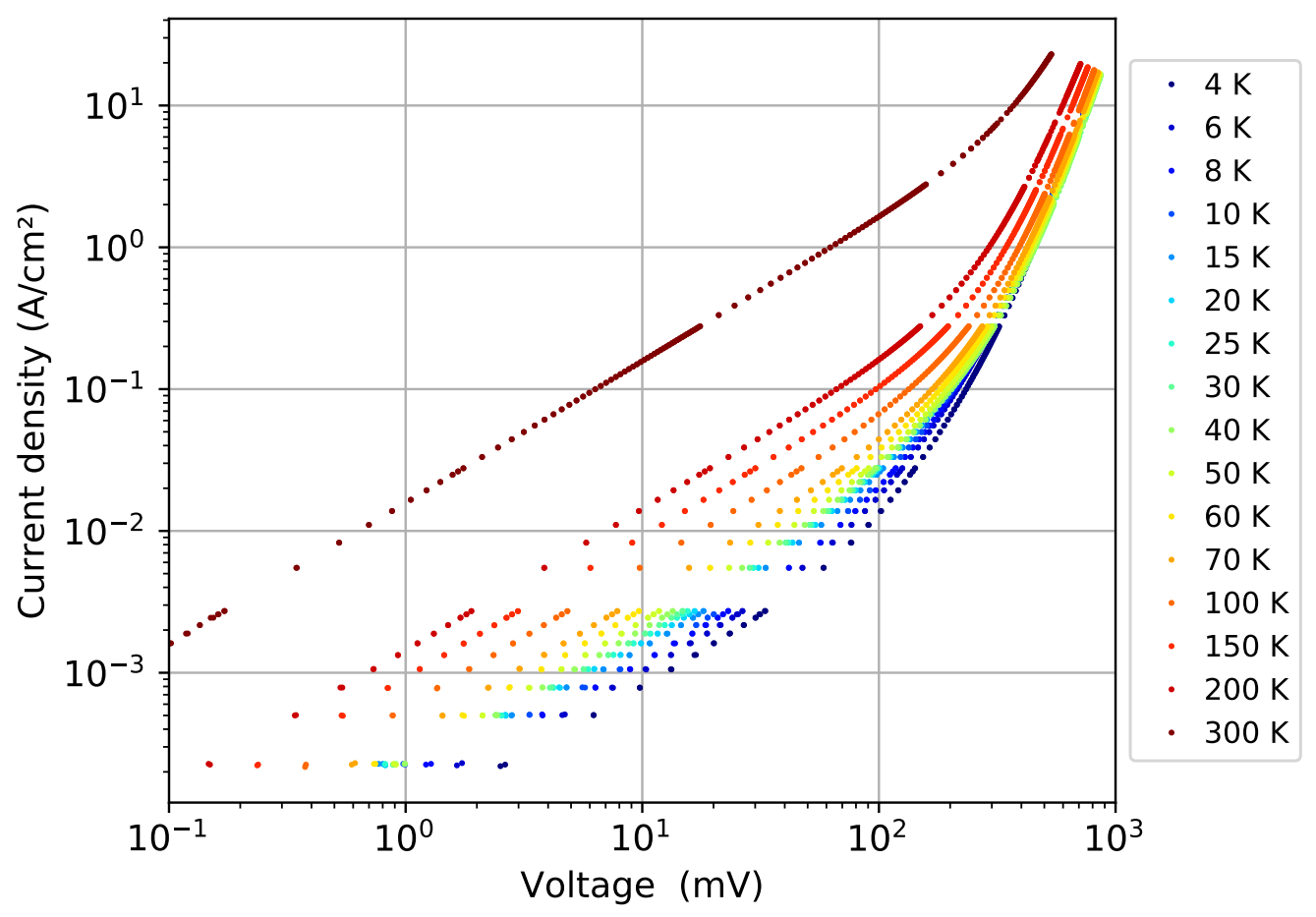

Figure 6.19: HR $(60 \mu \mathrm{m}): \mathrm{J}-\mathrm{V}$ curves in log-log scale at different temperatures from $10^{-1} \mathrm{mV}$ to $10^{3} \mathrm{mV}$.

Figure 6.20 shows the $\mathrm{J}-\mathrm{V}$ characteristics between $\pm 600 \mathrm{mV}$ of an LR device (active area $20 \mu \mathrm{m} \times 20 \mu \mathrm{m}$ ) at temperatures of $4 \mathrm{~K}$ and $300 \mathrm{~K}$. The lower resistance in this sample does not allow us to probe higher voltages $(>1 \mathrm{~V})$ without using a prohibitively large current, which overheats the tunnel junction.

The J-V characteristics of LR $(20 \mu \mathrm{m})$ in fig. 6.20 show the same cross over of curves at high voltage as in fig. 6.18, again corresponding to the lower resistance below $50 \mathrm{~K}$ in fig. 6.17 for the high current curves.

The J-V characteristics of the two samples appear similar at an initial glance, but there are some striking differences. The $300 \mathrm{~K}$ curve in fig. 6.20 is almost linear throughout the entire voltage range, but the $200 \mathrm{~K}$ shows clear non-linear behaviour at high voltage. In the LR devices, the cross- 


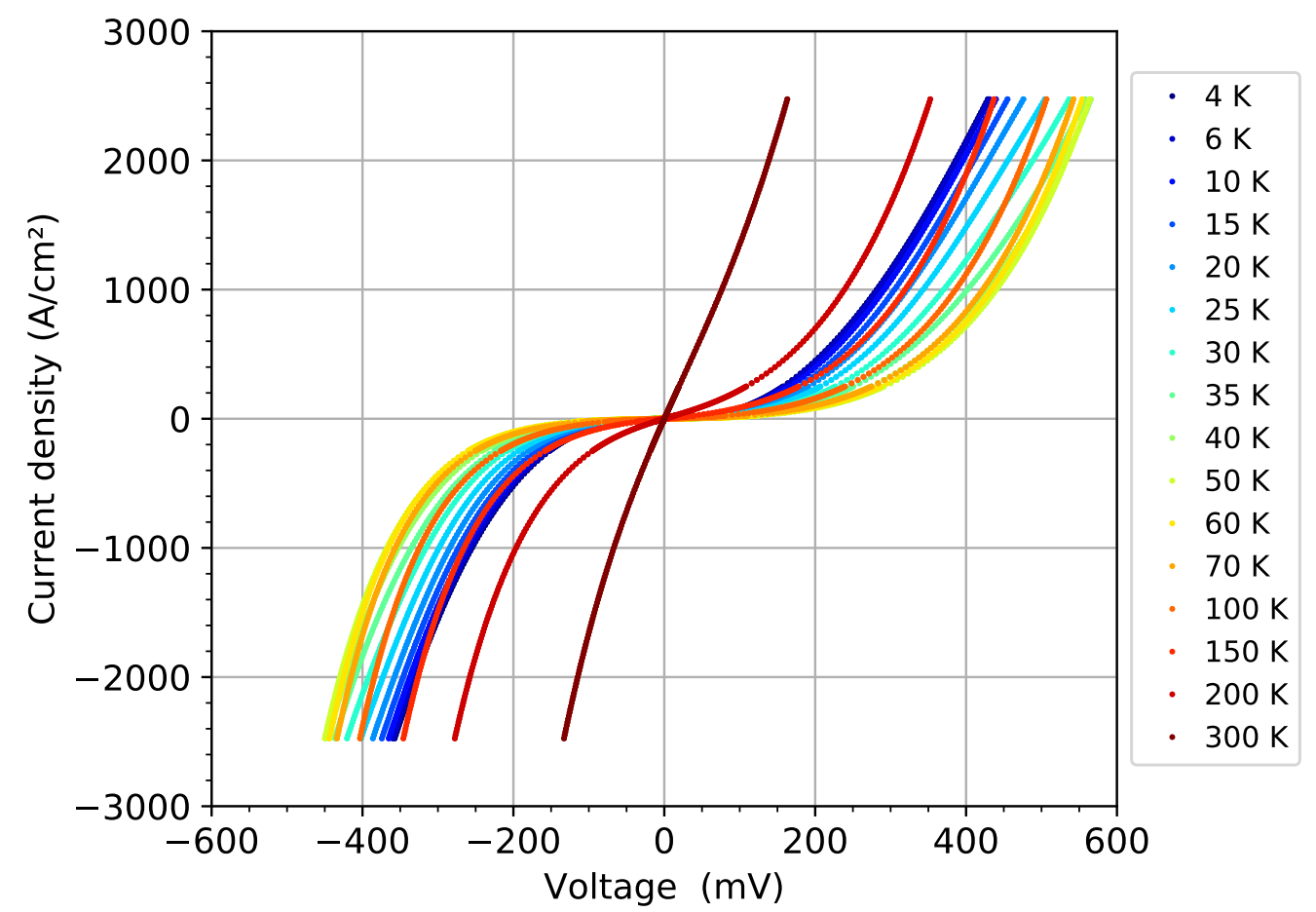

Figure 6.20: LR $(20 \mu \mathrm{m})$ : J-V curves at different temperatures between $\pm 600 \mathrm{mV}$.

over of the J-V curves at temperatures below $50 \mathrm{~K}$ is much stronger than in the HR devices. The J-V characteristics of the LR devices are also asymmetric, as expected from the explanation above.

The positive J-V characteristics from fig. 6.20 are shown on a logarithmic plot in fig. 6.21. Similar to the HR device in fig. 6.19 the LR device has linear J-V characteristics at low voltage and non-linear J-V characteristics at higher voltages. The device enters the non-linear region at lower voltages for lower temperatures. 


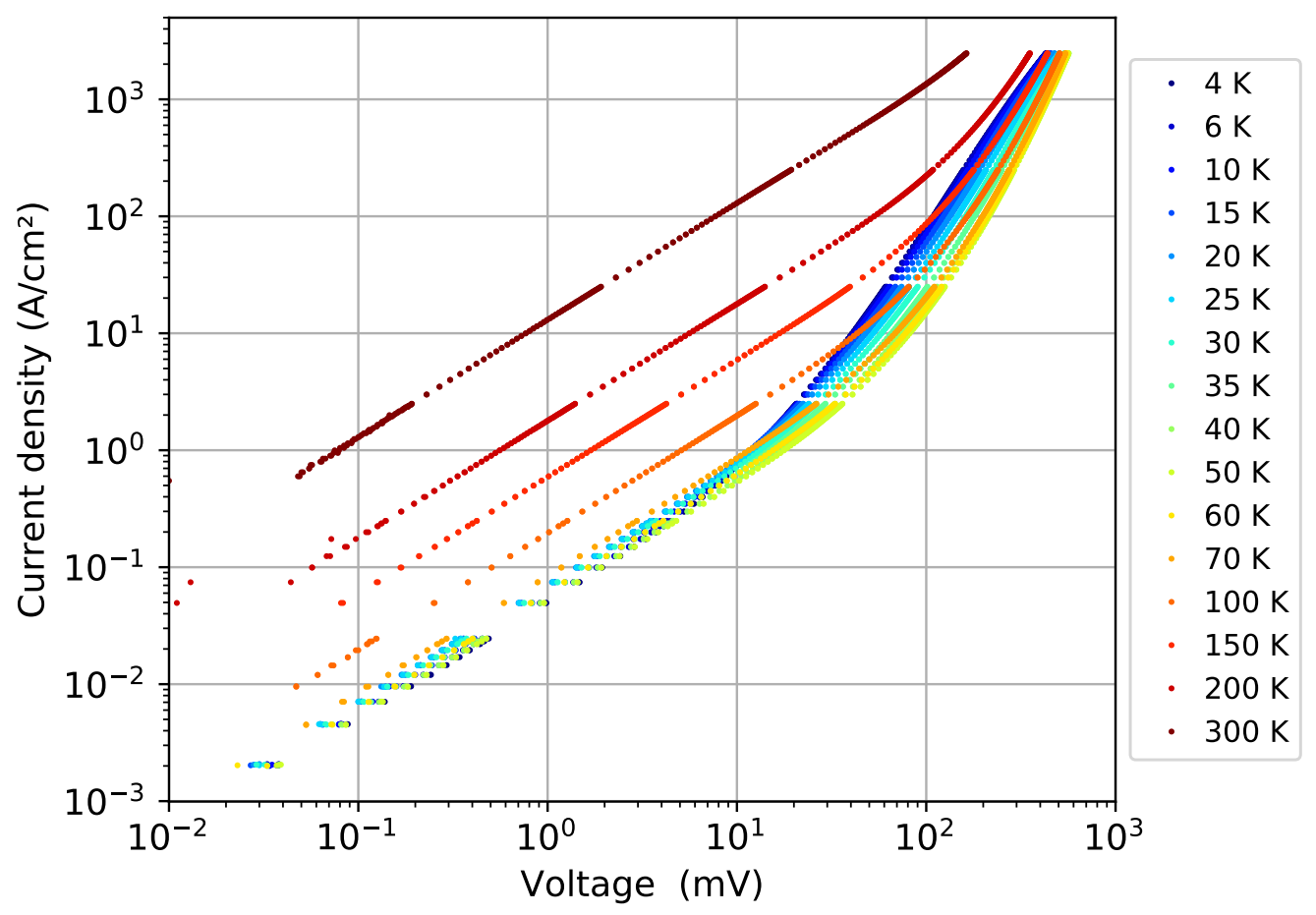

Figure 6.21: LR $(20 \mu \mathrm{m})$ : J-V curves in log-log scale at different temperatures from $10^{-2} \mathrm{mV}$ to $10^{3} \mathrm{mV}$. 


\section{Device size}

Within the model of tunnelling presented earlier, in fig. 6.1 one expects the tunnel current should be proportional to the active area, i.e. the current density-voltage curves should be the same regardless of the size of the active area. To illustrate this we show the I-V characteristics at $300 \mathrm{~K}$ and $4 \mathrm{~K}$ for devices of a variety of sizes.

In fig. 6.22 the I-V curves of two LR $40 \mu \mathrm{m} \times 40 \mu \mathrm{m}$ devices located at different parts of the substrate are shown for $300 \mathrm{~K}$ (red data points) and $4 \mathrm{~K}$ (blue data points). The I-V data at both temperatures are consistent for the two devices of the same area but from different places on the substrate which demonstrates that the devices behave consistently across the 2 inch substrate.

As devices of the same area behave similarly, we move on to compare I$\mathrm{V}$ characteristics across different active areas for sample LR. Five different area devices are shown in fig. 6.23 at $300 \mathrm{~K}$ (red data points) and $4 \mathrm{~K}$ (blue data points). In both the $300 \mathrm{~K}$ data sets and the $4 \mathrm{~K}$ datasets the current changes monotonically with device size. Smaller devices have a smaller current for the same applied voltage.

As the I-V characteristics seem to scale with device size, we plot the current density as a function of voltage to compare devices of different size, shown in fig. 6.24. The $300 \mathrm{~K}$ data in fig. 6.24 overlap well. The five $4 \mathrm{~K}$ data sets of the LR devices in fig. 6.24 do not overlap as well as the $300 \mathrm{~K}$ data sets in the linear region, but the different devices move towards the same J-V curve in the higher voltage region of the non-linear regime. In the linear region, the difference in current density changes monotonically with device size, which suggests that the conduction is more complicated at $4 \mathrm{~K}$.

Looking back at fig. 6.7, where the edges of the active area show inconsistencies, we may suspect that the conduction through the device is influenced by a region of different conductance around the edge of the ac- 


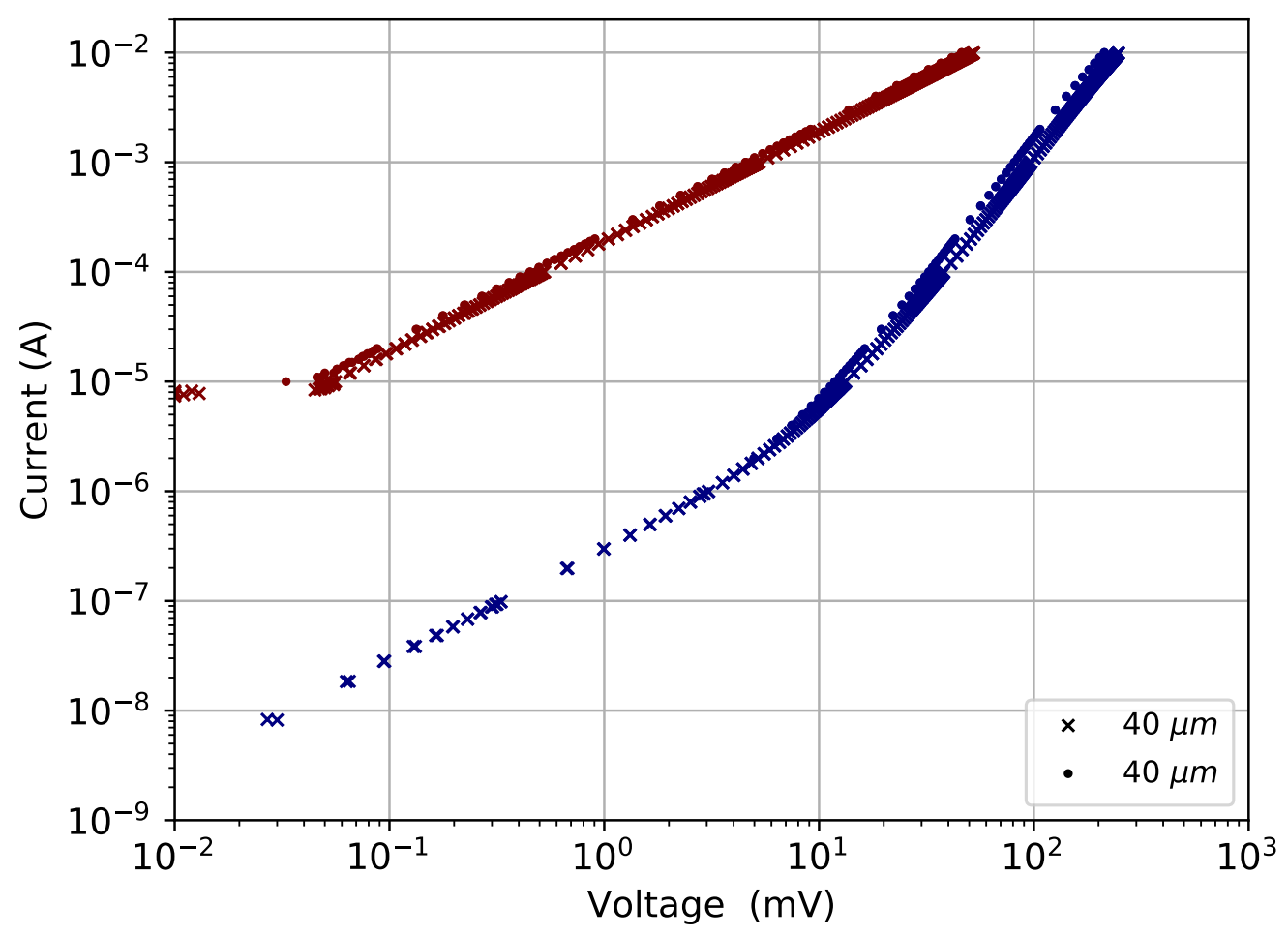

Figure 6.22: LR: I-V curves for two LR $1600 \mu \mathrm{m}^{2}$ devices at $300 \mathrm{~K}$ (red) and $4 \mathrm{~K}$ (blue). Legend shows device side length.

tive area. Figure 6.25 therefore shows the current for all five LR devices scaled by the side length of the active area. The $300 \mathrm{~K}$ data, as expected from the excellent scaling with the area, does not scale well with the side length. For the $4 \mathrm{~K}$ data, the overlap in the linear regime is excellent when scaled by the side length. The overlap of curves in the non-linear regime is similar when scaled by the area as when scaled by the side length.

The dependencies on the area and circumference in the linear regime for the $4 \mathrm{~K}$ data suggest a leakage path around the circumference of the active area in the LR devices which influences the low-temperature data more than the high-temperature data. The low-temperature data indicates that the total conduction is dominated by conduction around the edge in the linear region while in the non-linear region it is a mixture of the area 
conduction and the circumference conduction.

To compare the LR devices to the HR devices, the I-V characteristics for six different HR devices are shown in fig. 6.26 for $300 \mathrm{~K}$ (red data points) and $4 \mathrm{~K}$ (blue data points). In contrast to the LR devices, the $300 \mathrm{~K}$ datasets overlap in the I-V plot, which means they do not scale with device size. Given the complete lack of area dependence at $300 \mathrm{~K}$ we suggest that the resistance of the GaN area restriction layer is similar to the device resistance at room temperature and all HR devices, therefore, have the same total effective area at $300 \mathrm{~K}$ due to the conductive path through the area restriction layer. The $4 \mathrm{~K}$ curves do not overlap as the $300 \mathrm{~K}$ curves, but

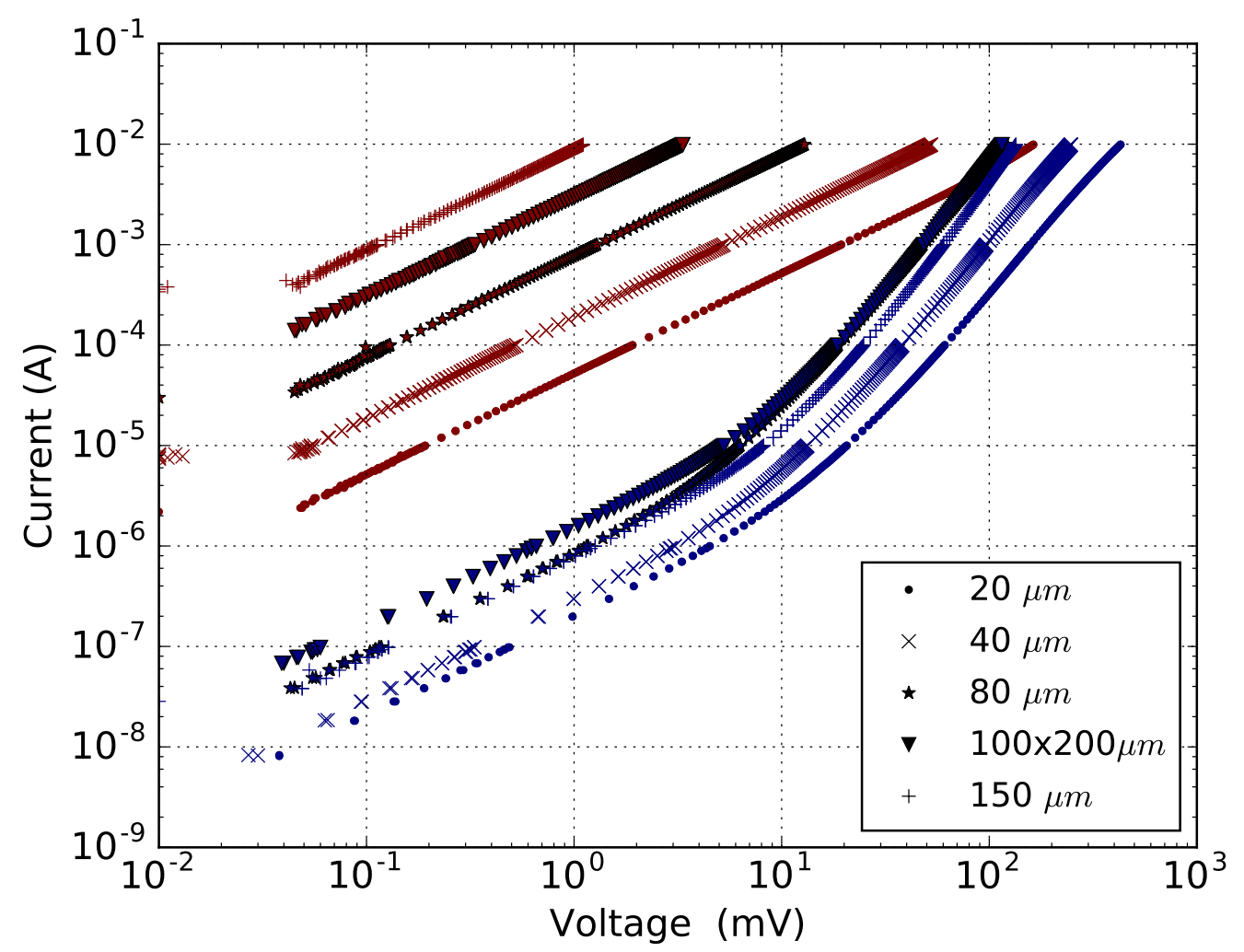

Figure 6.23: LR: I-V curves from five LR device for with different area devices at $300 \mathrm{~K}$ (red) and $4 \mathrm{~K}$ (blue). Legend shows device side length, except one which is rectangular and shows both side lengths. 


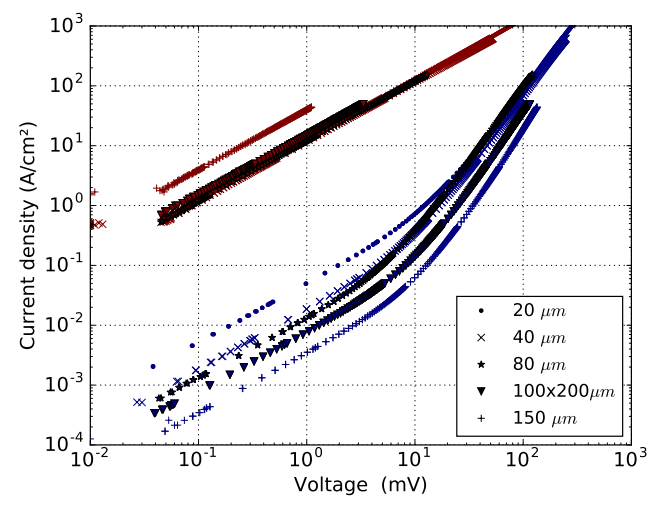

Figure 6.24: LR: J-V curves from five LR devices for with different area devices at $300 \mathrm{~K}$ (red) and $4 \mathrm{~K}$ (blue). Legend shows device side length, except one which is rectangular and shows both side lengths.

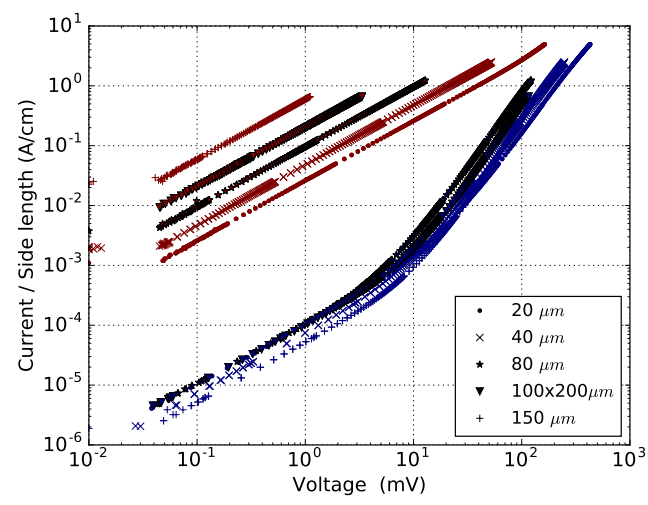

Figure 6.25: LR: I/L-V curves from five LR devices for with different area devices at $300 \mathrm{~K}$ (red) and $4 \mathrm{~K}$ (blue). Legend shows device side length, except one which is rectangular and shows both side lengths.

also does not scale with device size like the LR devices. At lower temperature, the resistance of the GaN restriction layer is large enough that it no longer provides a conduction path. Furthermore, the non-size scaled behaviour at $4 \mathrm{~K}$ suggests that the HR device contain hotspots or pinholes which become important at low temperature.

In a tunnel junction, the electrical transport due to tunnelling cannot easily be distinguished from transport through a pinhole [79,80]. The fraction of the electrical transport through a barrier which is due to pinhole mediated transport increases with decreasing temperature and increasing voltage [81]. Depending on the exact shape, composition, and position of the pinholes the behaviour is likely to change when the device electrodes become magnetic.

The LR devices in fig. 6.23 do not show as clear evidence of pinholes in the I-V characteristics as the HR devices in fig. 6.26 , but the conduction around the circumference could be interpreted as a hotspot. The I-V char- 


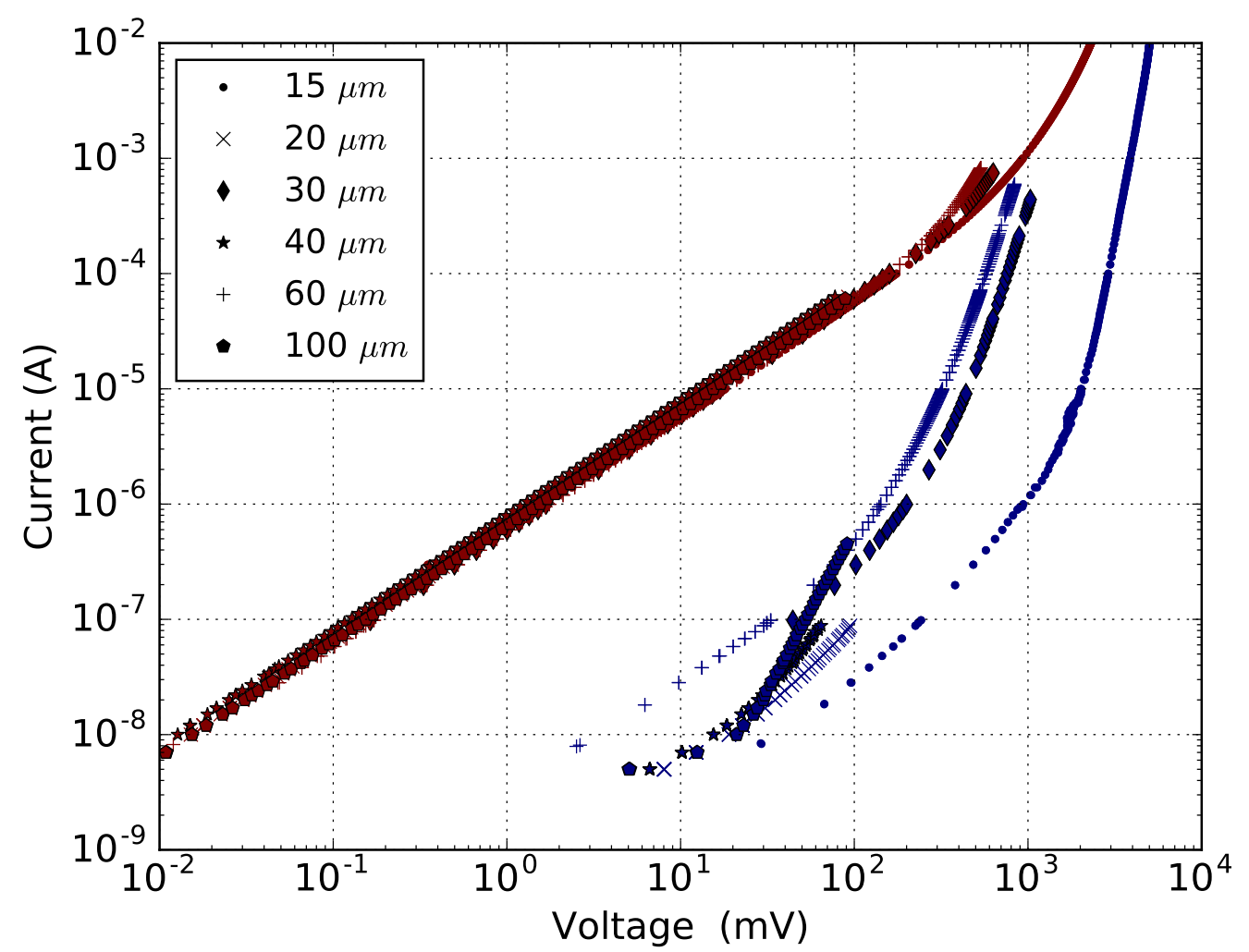

Figure 6.26: HR: I-V curves from six HR device for with different area devices at $300 \mathrm{~K}$ (red) and $4 \mathrm{~K}$ (blue). The legend shows device side length.

acteristics of the LR devices scale well with the area at high temperature as well as in the non-linear regime at low temperature. In the linear regime at low temperature the current scales well with the side length, suggesting a thin region around the circumference of the device with higher conductivity, corroborated by SEM imaging. This region of higher conductivity may arise from a thinner GaN tunnel barrier around the edge, as when the tunnel stack is deposited it has to bend to accommodate the shape of the GaN active area patterning. 


\section{J-V slope}

After studying the $\mathrm{J}-\mathrm{V}$ characteristics and the scaling with device size, we will investigate the rate of change of the current density with voltage and temperature to see if the behaviour can be understood using a simple power law in the high voltage region. To study this, we plot the slope of the J-V curve in the log-log plot as a function of voltage. A slope of one in the log-log plot corresponds to the linear J-V regime.

The slope of the log-log plot of the LR $(20 \mu \mathrm{m})$ device is shown in fig. 6.27, corresponding to the log-log plot in fig. 6.21. As the temperature decreases, the J-V characteristics become non-linear at lower voltages.

At $100 \mathrm{mV}$ the slope of the $2 \mathrm{~K}$ curve turns over and becomes lower again. All temperatures below $40 \mathrm{~K}$ show a turnover of the slope, but the turnover becomes very visible in fig. 6.27 for temperatures below $30 \mathrm{~K}$. The turnovers occur at a similar voltage in all LR devices regardless of size.

The noise in the figure mainly arises from calculating the slope using adjacent points, but the overall trends are easily observed despite the noise. The 'spikes' in the noise (for example at $20 \mathrm{mV}$ in the $4 \mathrm{~K}$ curve) correspond to a change in the step size in the current settings, as a larger step between currents reduces the noise in the calculation of the derivative. The change in current steps can more easily be seen in fig. 6.21, and the voltages at which the current step changes corresponds to the 'spikes' in the slope calculations. 


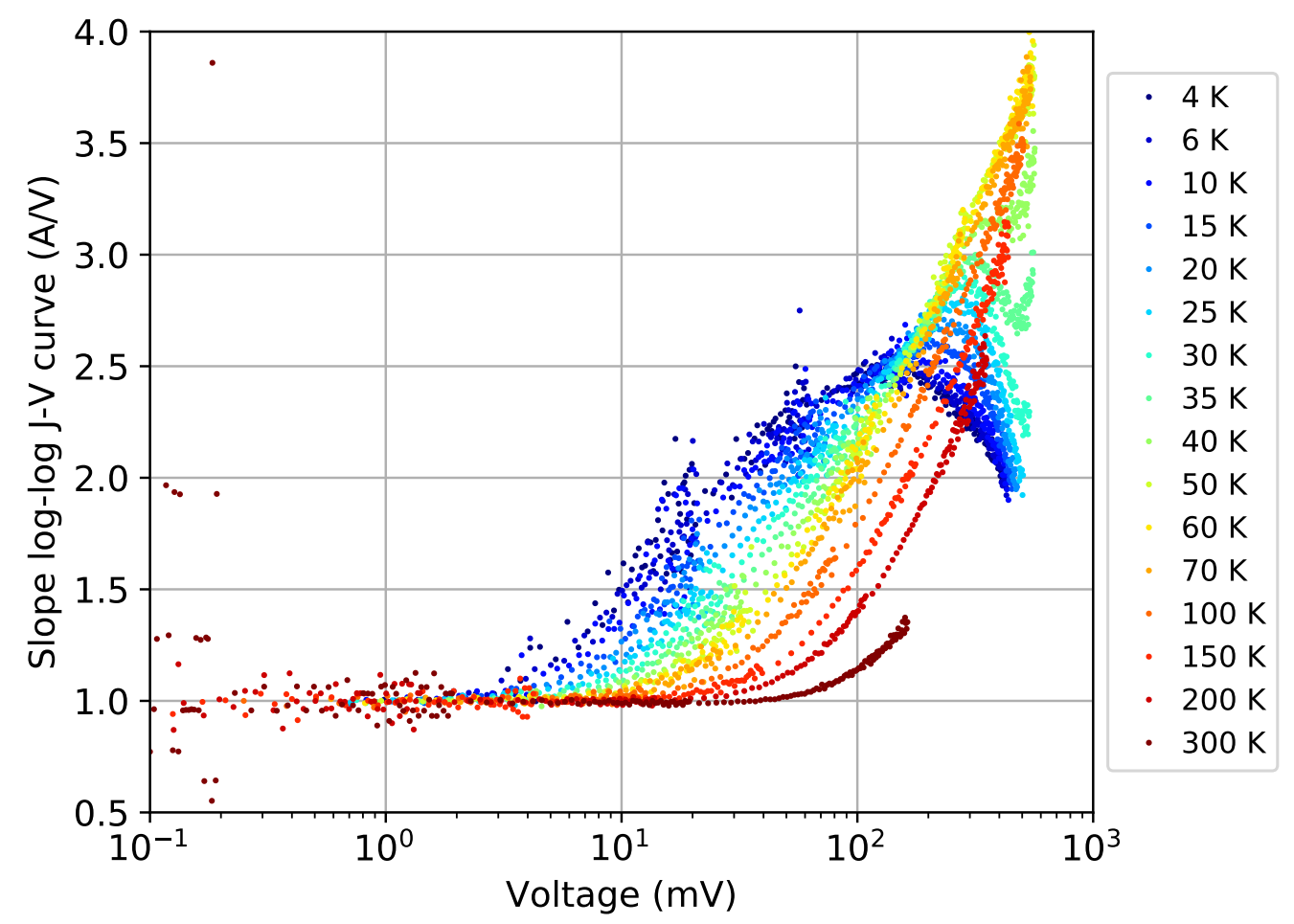

Figure 6.27: LR $(20 \mu \mathrm{m})$ : Slope of $\log -\log \mathrm{J}-\mathrm{V}$ curves at different temperatures from $10^{-1} \mathrm{mV}$ to $10^{3} \mathrm{mV}$.

The slope does not become constant for high voltages, meaning the electrical behaviour is more complicated than can be described by a power law, as expected for a MTJ. In the next section, we will describe and fit a Simmons model to the J-V curves. 


\subsubsection{Simmons model of J-V characteristics}

There are a number of models of the behaviour of tunnel junctions. A lot of the more well-known models, such as the Simmons model and the Brinkman's model, are based on the Wentzel-Kramers-Brillouin (WKB) approximation for tunnelling probability calculation. These models only describe direct tunnelling and do not include the effects of defects, scattering, or pinholes.

The Simmons model is the most commonly applied model in literature to extract parameters such as barrier width and barrier height. The Simmons model, while built on the WKB approximation, is formulated in terms of experimentally measurable factors such as the current density, bias voltage, barrier height, and barrier width. The model can account for different scenarios such as a rectangular barrier, a trapezoidal barrier, temperature dependence, and image charges. [108]

Starting from the Hamiltonian of a tunnelling electron and using the WKB approximation of tunnelling probability the current density through a magnetic tunnel junction when the applied voltage is less than the barrier height is described in Simmons model [108] as

$$
\begin{aligned}
J(V, T=0)= & \frac{6.2 \cdot 10^{10}}{s^{2}} . \\
& {\left[\left(\phi_{0}-V / 2\right) \exp \left(-1.025 s\left(\phi_{0}-V / 2\right)^{\frac{1}{2}}\right)-\right.} \\
& \left.\left(\phi_{0}+V / 2\right) \exp \left(-1.025 s\left(\phi_{0}+V / 2\right)^{\frac{1}{2}}\right)\right]
\end{aligned}
$$

where $V$ is the applied voltage $(\mathrm{V}), T$ is the temperature $(\mathrm{K}), s$ is the barrier width $(\AA), \phi_{0}$ is the barrier height $(\mathrm{eV})$.

Often a small correction is made for the image forces arising in the electrodes where the barrier height is replaced by an effective barrier height, $\phi_{1}$.

$$
\phi_{1}=\phi_{0}-(V / 2 s)\left(s_{1}+s_{2}\right)-\frac{5.75}{K\left(s_{2}-s_{1}\right)} \ln \left[\frac{s_{2}\left(s-s_{1}\right)}{s_{1}\left(s-s_{2}\right)}\right]
$$


where $\mathrm{K}$ is the dielectric constant/relative permittivity of the barrier material, and $s_{1}$ and $s_{2}$ are barrier thickness parameters calculated as

$$
\begin{aligned}
& s_{1}=6 / K \phi_{0} \\
& s_{2}=s\left[1-\frac{46}{3 \phi_{0} K s+20-2 V K s}\right]+6 / K \phi_{0}
\end{aligned}
$$

Combining gives the generalised case for tunnelling through a rectangular barrier with image forces and with an applied voltage below the barrier height

$$
\begin{aligned}
J(V, T=0)= & \frac{6.2 \cdot 10^{10}}{\Delta s^{2}} \\
& {\left[\phi_{1} \exp \left(-1.025 \Delta s \phi_{1}^{\frac{1}{2}}\right)-\right.} \\
& \left.\left(\phi_{1}+V\right) \exp \left(-1.025 \Delta s\left(\phi_{1}+V\right)^{\frac{1}{2}}\right)\right]
\end{aligned}
$$

where $\Delta s=s_{2}-s_{1}$ is the adjusted barrier width $(\AA)$.

One can make a correction for the temperature as follows

$$
\frac{3 \cdot 10^{-9} \cdot \Delta s^{2} T^{2}}{\phi_{0}-V / 2}
$$

which only applies when the barrier is symmetric. This gives the final model as

$$
J(V, T)=J(V, 0)\left[1+\frac{3 \cdot 10^{-9} \cdot \Delta s^{2} T^{2}}{\phi_{0}-V / 2}\right]
$$

This model assumes symmetric metallic electrodes which is not the case for the devices in this thesis. However, the Simmons model is often quoted as a standard measure, in published literature, of the quality of a tunnel junction. Therefore we have attempted to fit the Simmons model to the data as a comparison to many MTJs in published literature. We will use the parameters extracted from the Simmons fit as a rough comparison between device, i.e. a device with a larger extracted barrier width is probably wider than a device with a smaller extracted barrier width but the exact barrier parameters are unlikely to match. 
To find the global minimum of the Simmons fit, a python script was written which tests all values for the barrier height and barrier width in a specified region and show how well they fit the data in a least squares fit for that pair of parameters.

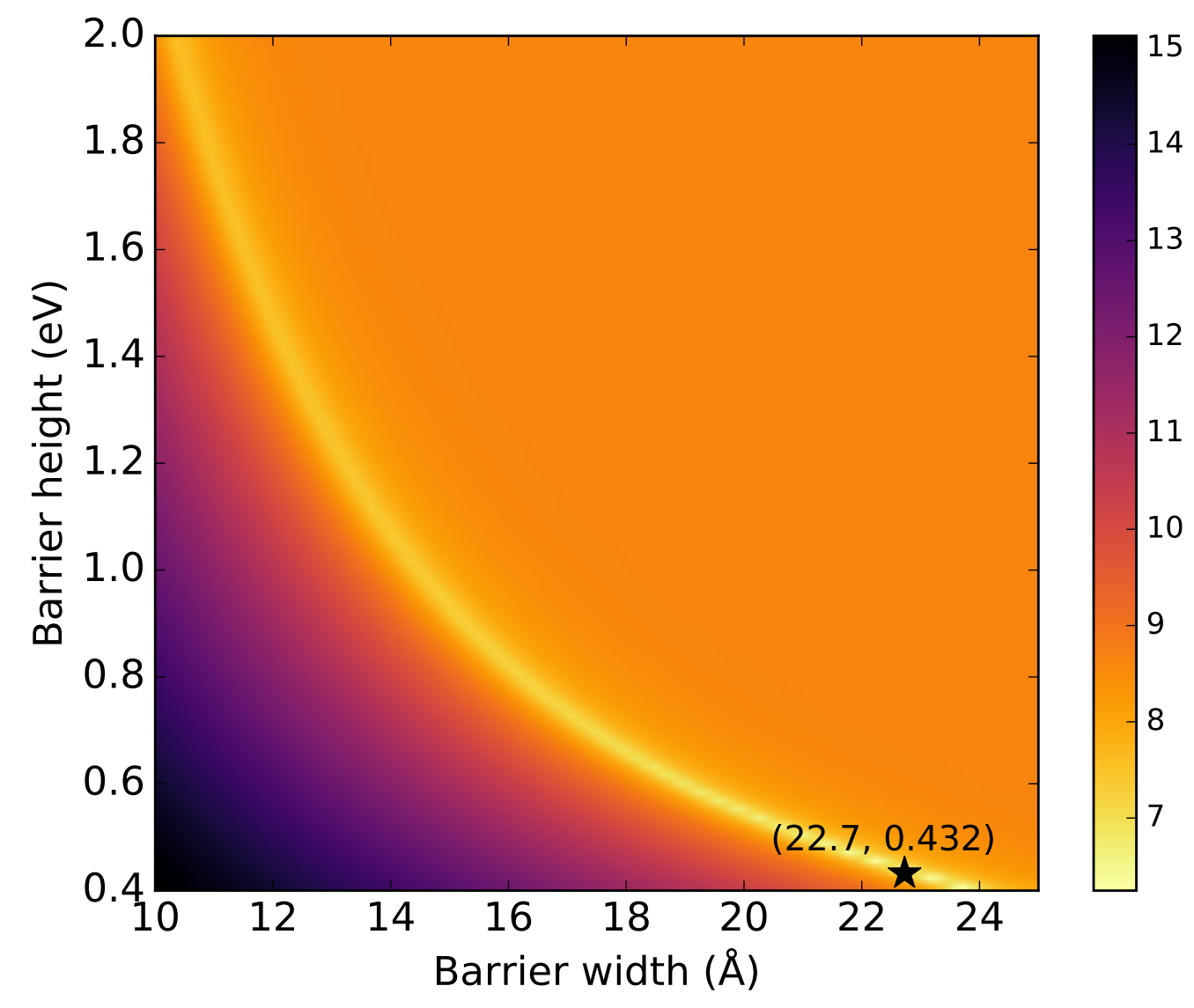

Figure 6.28: LR $(20 \mu \mathrm{m})$ : The $\log 10$ of the residual error in fitting the experimental J-V characteristics to a Simmons model using different barrier parameters. The colour map corresponds to the log 10 of the least squares fitted error. The star marks the fitting parameters resulting in the lowest error.

In fig. 6.28 we see the $\log 10$ of the residual error from the least squares fit between the Simmons fit evaluated at each set of parameters and the 


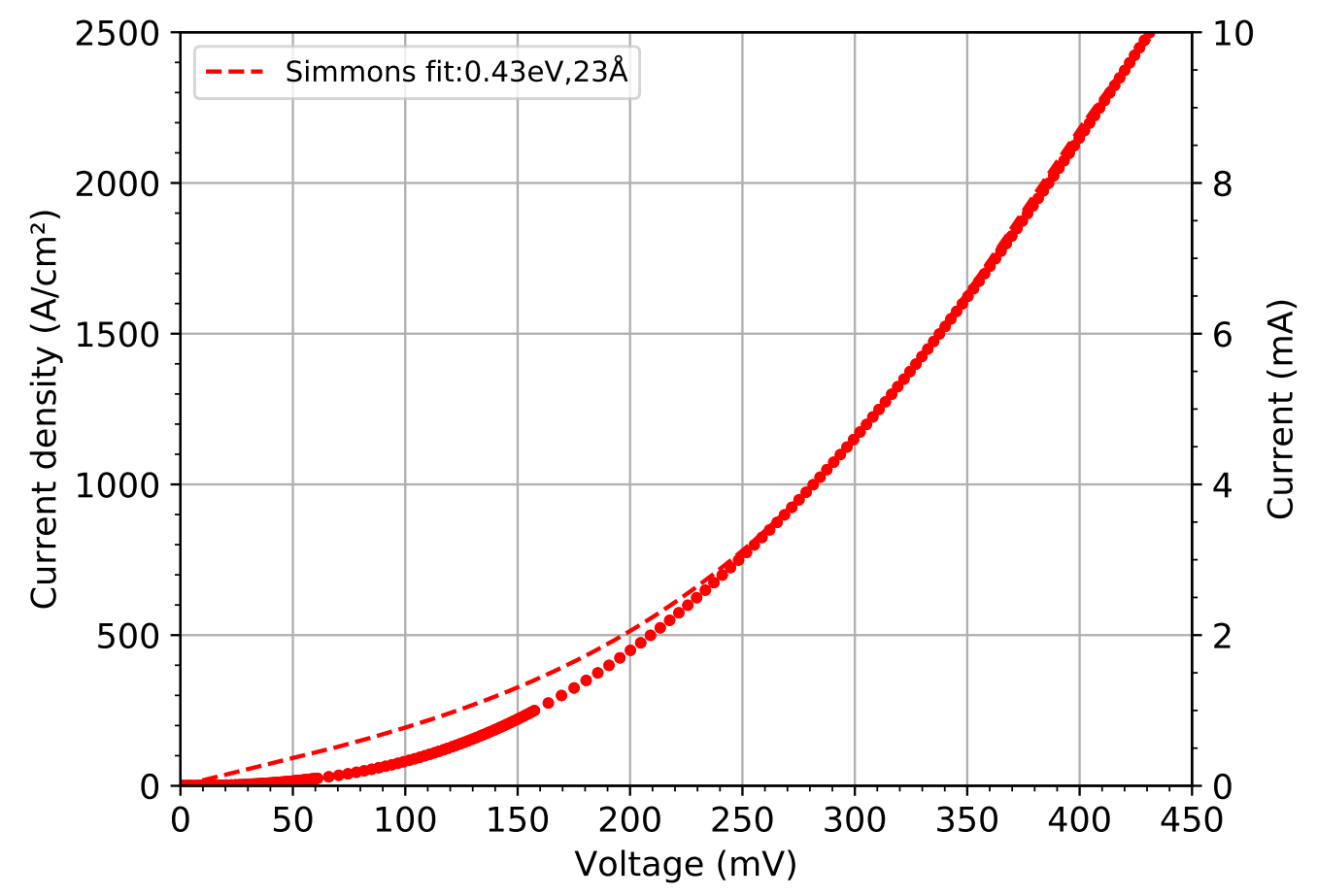

Figure 6.29: LR $(20 \mu \mathrm{m}): \mathrm{J}-\mathrm{V}$ curves of the $4 \mathrm{~K}$ data fitted with a Simmons model with values chosen from the minimum in fig. 6.28.

experimentally measured J-V characteristics. The black star (with coordinates marked) marks the lowest residual error and hence the best fit. In the figure, we see a colour gradient where light yellow indicates the best fit and black indicates worst fit to the data. There is a valley of best fit as evidenced by the light yellow band suggesting the experimental data can be fitted with a Simmons model for a variety of barrier parameters without a large increase in the residual error.

In fig. 6.29 the positive J-V data at $4 \mathrm{~K}$ from the fig. 6.20 can be seen together with a Simmons model fit with a barrier width of $2.3 \mathrm{~nm}$ and $0.43 \mathrm{eV}$. The fitted barrier thickness is accurate to within $0.5 \mathrm{~nm}$ of the designed barrier thickness.

To compare the fit in the LR devices with a HR device, fig. 6.30 shows the J-V characteristics at $4 \mathrm{~K}$ from device HR $(60 \mu \mathrm{m})$ with a Simmons fit 


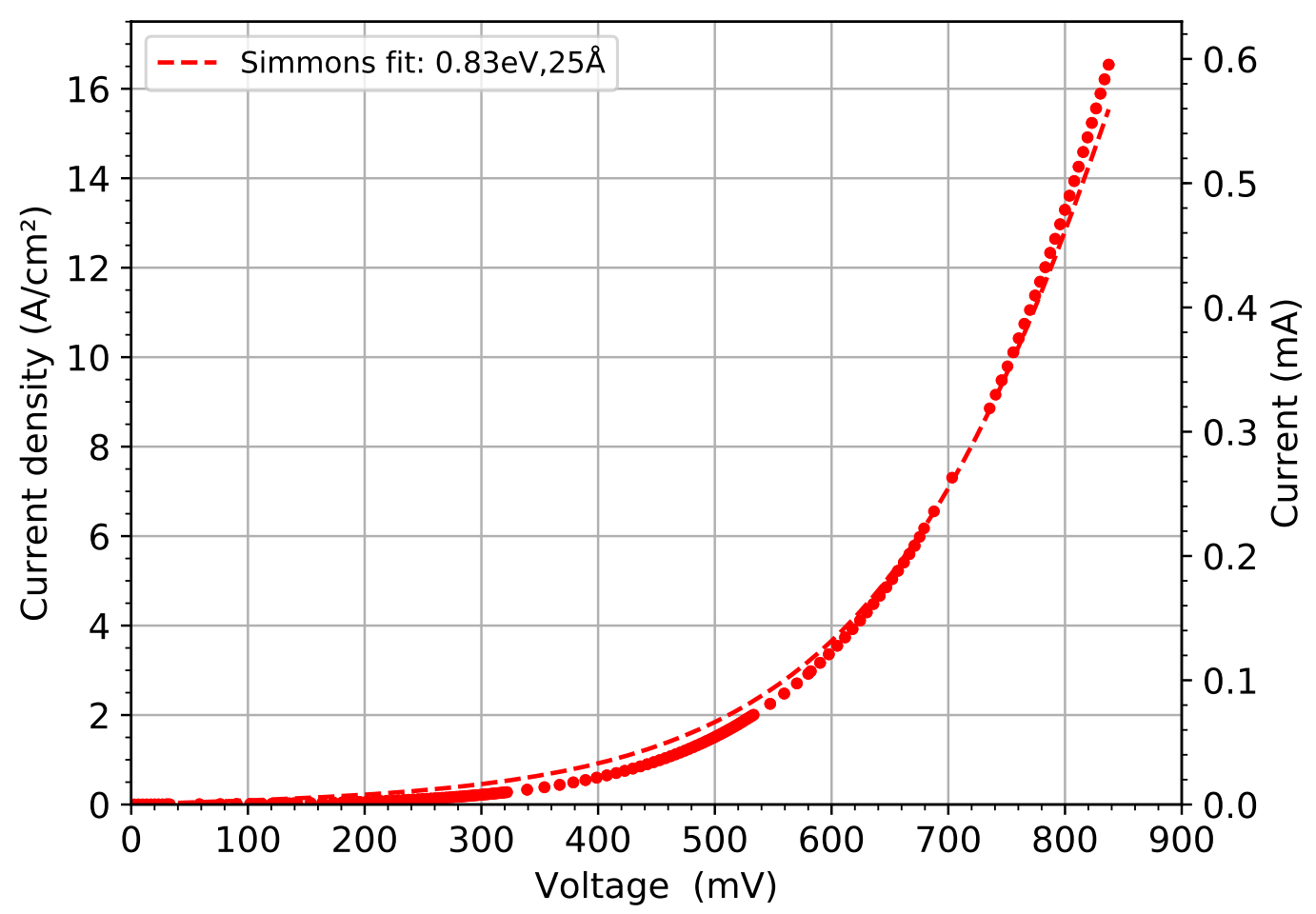

Figure 6.30: HR $(60 \mu \mathrm{m}): \mathrm{J}-\mathrm{V}$ curves of the $4 \mathrm{~K}$ data fitted with a Simmons model.

performed with a global minimisation as above. The fitted barrier height $(0.83 \mathrm{eV})$ is very different to the fit in fig. 6.29 while the barrier width $2.5 \mathrm{~nm}$ is again within $0.5 \mathrm{~nm}$ of the designed barrier thickness. The low voltage percentage misfit to the Simmons fit at $4 \mathrm{~K}$ is similar to the LR devices, but it appears smaller in fig. 6.30 due to the fast rise in current density above $600 \mathrm{mV}$ in the HR device.

The Simmons model estimates barrier widths within $0.5 \mathrm{~nm}$ of the designed barrier thickness from growth conditions for all devices, and with large difference in barrier heights between HR and LR devices despite both being GaN barriers. If the Simmons model is restricted to fit the same barrier height for both samples, but with a free barrier width, the fit is much worse than the two fits presented above.

By making a Simmons fit to different devices within the same sample, 
for example multiple HR devices, and getting similar barrier parameters supports that the growth of the devices is consistent across the 2 inch substrate.The barrier height in both HR and LR devices is much lower than the expected barrier height of $1.5 \mathrm{eV}$ from comparing with the barrier heights in GdN/GaN/GdN and GdN/AlN/SmN MTJ [9,73,99]. This suggests the tunnelling is not dominated by direct tunnelling across the barrier [83]. 
To conclude the electrical properties of the MTJs in no applied field we have found:

1. The resistance of the MTJs is dominated by the tunnel barrier.

2. The tunnel currents change at $\mathrm{T}_{C}$ of the two electrodes, evidenced by increased resistance around $30 \mathrm{~K}$ and $50 \mathrm{~K}$.

3. The I-V characteristics are non-linear and asymmetric.

4. The LR devices have a thinner region around the edge of the active area which dominates the transport in the linear I-V region.

5. The HR devices potentially pinholes in the barrier.

6. The J-V characteristics cannot be described with a power law.

7. The J-V characteristics are not well described by the Simmons model, suggesting indirect tunnelling across the barrier. 


\subsubsection{Magnetoresistance}

We now move on to a investigate the electrical characteristics of the MTJ devices in a magnetic field. Unless otherwise stated the field is always applied in-plane of the device.

We start by plotting the temperature dependent resistance of LR $(20 \mu \mathrm{m})$ at $10 \mu \mathrm{A}$ in different applied fields in fig. 6.31, with the inset showing the same measurement for the same sample but at $10 \mathrm{nA}$. The two currents, $10 \mu \mathrm{A}$ and $10 \mathrm{nA}$, correspond to the onset of non-linearity and the linear regime in the I-V characteristics, respectively.

The device in fig. 6.31 shows a strong magnetic response. As the field is increased the $50 \mathrm{~K}$ feature reduces in height, relative to both the resistance at $2 \mathrm{~K}$ and $200 \mathrm{~K}$. The $50 \mathrm{~K}$ feature appears to shift to higher temperatures for larger applied fields. As $\mathrm{T}_{C}$ shifts to a higher temperature in an applied field, this supports the suggestion that the tunnel current changes as the GdN band structure becomes spin split. Note that a small magnetic response persists up to $175 \mathrm{~K}$ at $9 \mathrm{~T}$ but in $2 \mathrm{~T}$ the magnetic response is minimal above $100 \mathrm{~K}$.

When the $50 \mathrm{~K}$ feature is modifieds by an applied field it is also possible to distinguish the smaller feature around $25 \mathrm{~K}$ more clearly, again supporting a change in the tunnel current from the band structure change when the $\mathrm{SmN}$ electrode becomes spin split.

The largest resistance change in fig. 6.31 when applying a field occurs around $50 \mathrm{~K}$. A different way to study the change in resistance is to sweep the field at a constant temperature to look for potential hysteresis and switching.

In fig. 6.32 the magnetoresistance as a function of field of LR (40 $\mu \mathrm{m})$ at $1 \mu \mathrm{A}$ (in the linear $\mathrm{J}-\mathrm{V}$ regime) is plotted for different temperatures. The resistance of the device decreases as the magnetic field is increased at all temperatures. All temperatures below $70 \mathrm{~K}$ show a cusp-like resistance change at low absolute field, while the higher temperatures show a 


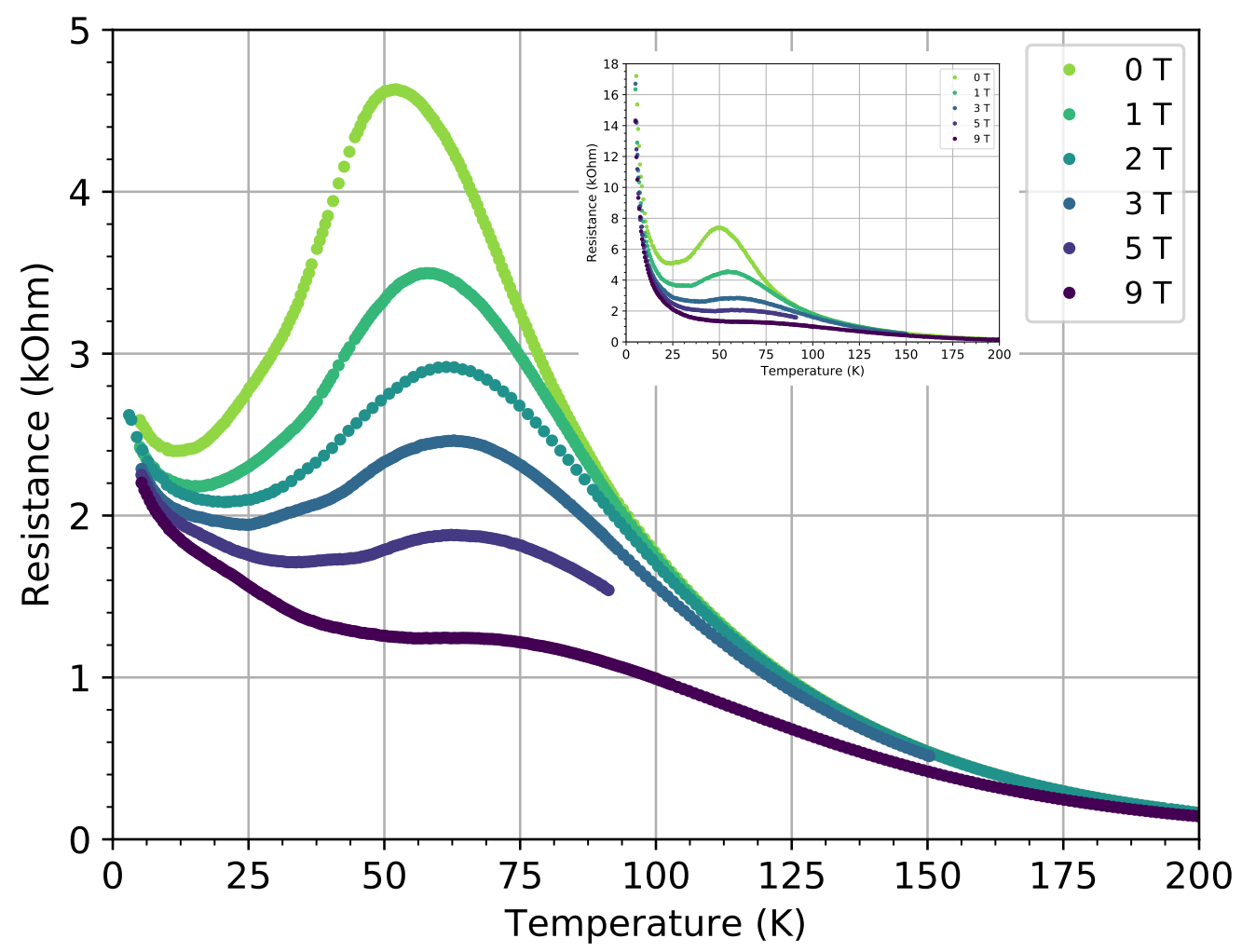

Figure 6.31: LR $(20 \mu \mathrm{m})$ : Resistance vs temperature measured at $10 \mu \mathrm{A}$ in different applied fields. Inset: Same sample but measured at $10 \mathrm{nA}$ showing the strong low temperature resistance increase for all fields. 
smoother resistance change. As this is around $\mathrm{T}_{C}$ of $\mathrm{GdN}$, we attribute the cusp-like shape to ferromagnetic ordering in the GdN electrode.

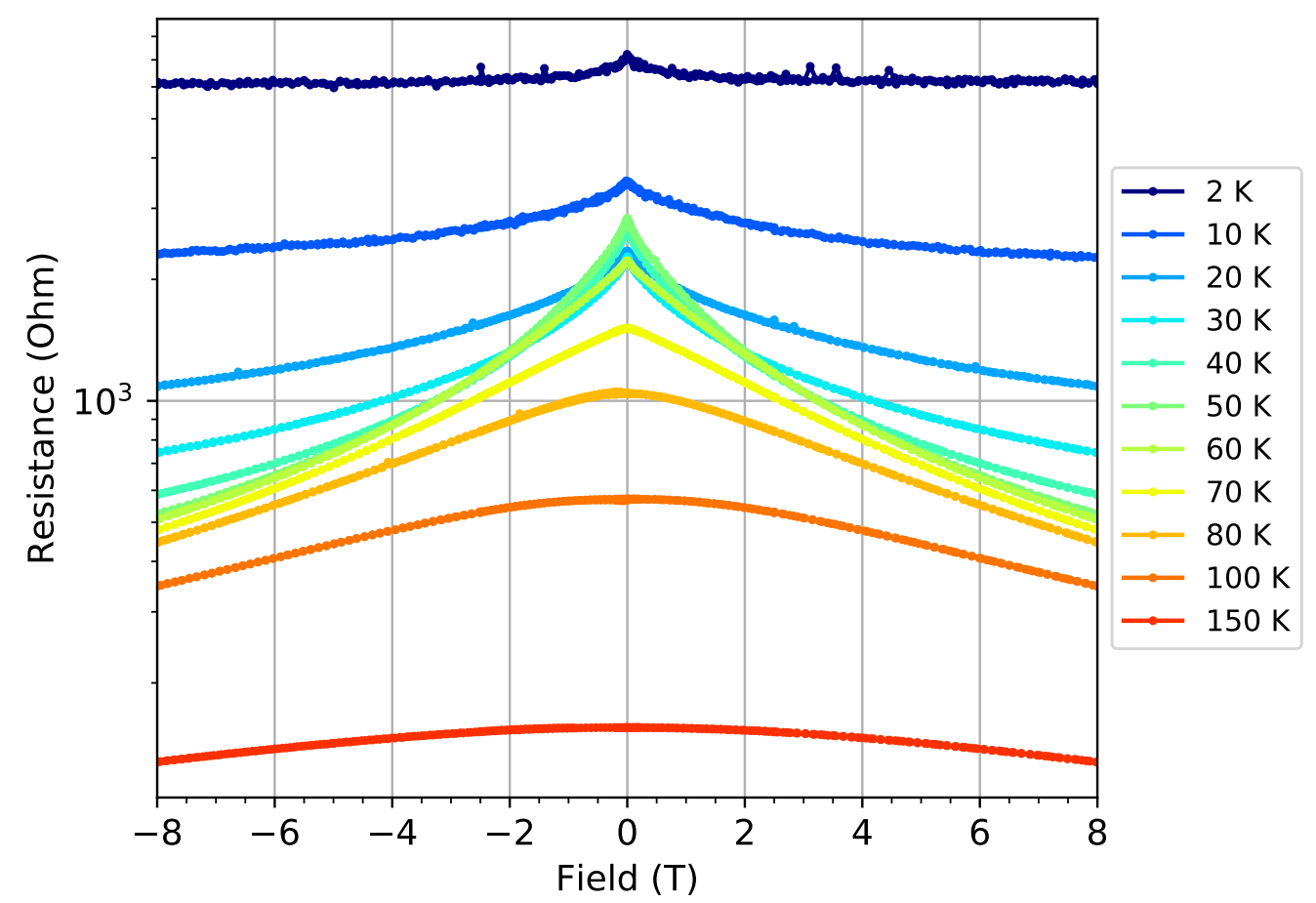

Figure 6.32: LR (40 $\mu \mathrm{m})$ : Log-plot of magnetoresistance at $1 \mu \mathrm{A}$ at different temperatures.

As the resistance magnitude is quite different at the different temperatures, we instead calculate the JMR using eq. (6.9) and plot fig. 6.33 against the applied magnetic field. This allows us to study the relative shape of the curves as well as the magnitude of the resistance change.

$$
J M R(B)=\frac{R(B)-R(\max )}{R(\min )} \cdot 100
$$

where $R(\max )$ and $R(\min )$ are the maximum and minimum resistances of the device respectively, found close to $0 \mathrm{~T}$ and at $8 \mathrm{~T}$, respectively. We 
also define the tunnel magnetoresistance (TMR) in our devices as the maximum JMR, which means

$$
T M R=J M R(8 T)
$$

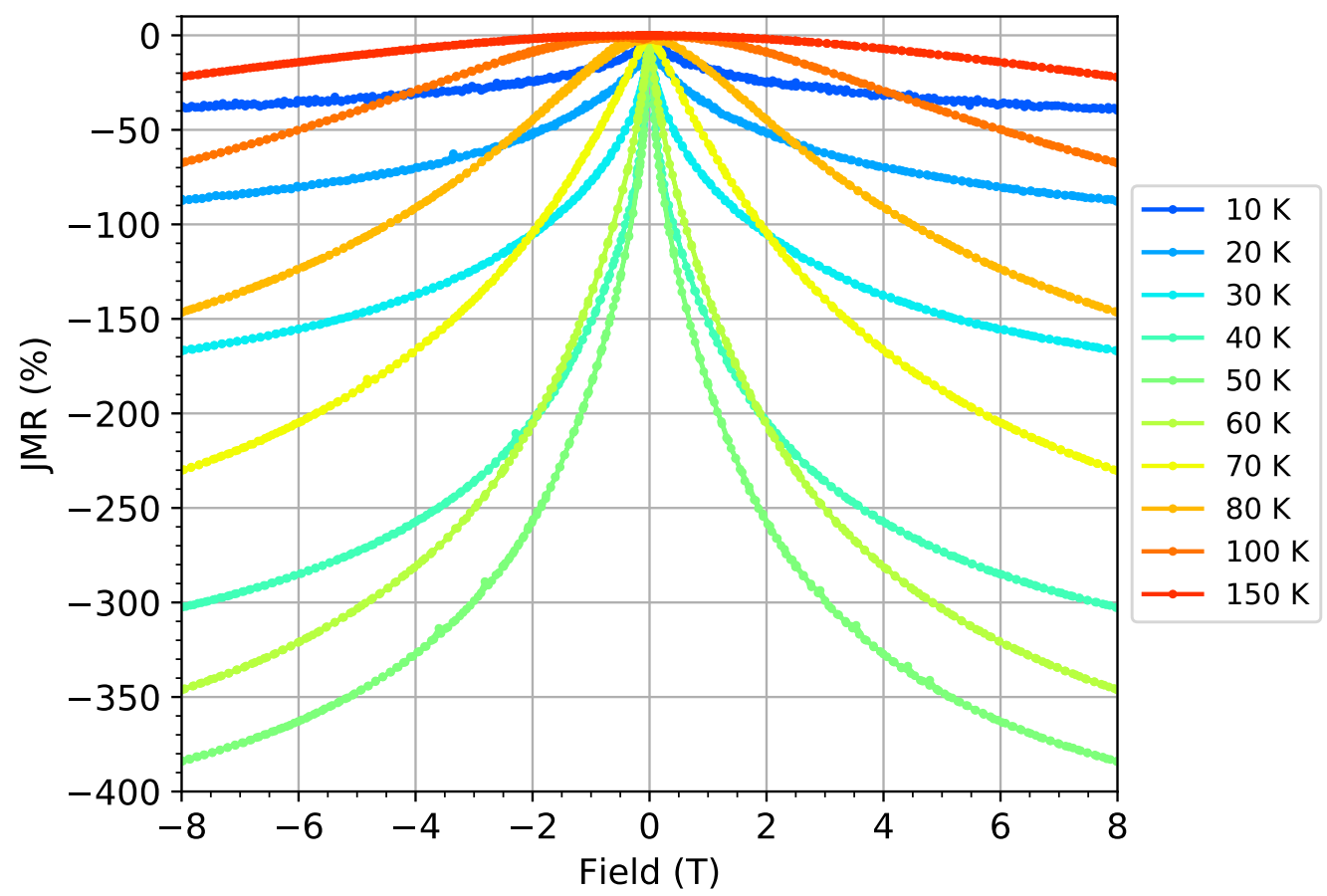

Figure 6.33: LR $(40 \mu \mathrm{m})$ : JMR at different temperatures. All are measured at $1 \mu \mathrm{A}$. 
To compare the LR devices to a HR device, we plot the resistance with changing magnetic field for different temperatures of $\mathrm{HR}(20 \mu \mathrm{m})$ at $10 \mathrm{nA}$ (in the linear J-V regime) in fig. 6.34. The HR devices show similar overall behaviour with the applied magnetic field, with a cusp-like low field feature below $70 \mathrm{~K}$ and a smooth resistance decrease at $70 \mathrm{~K}$.

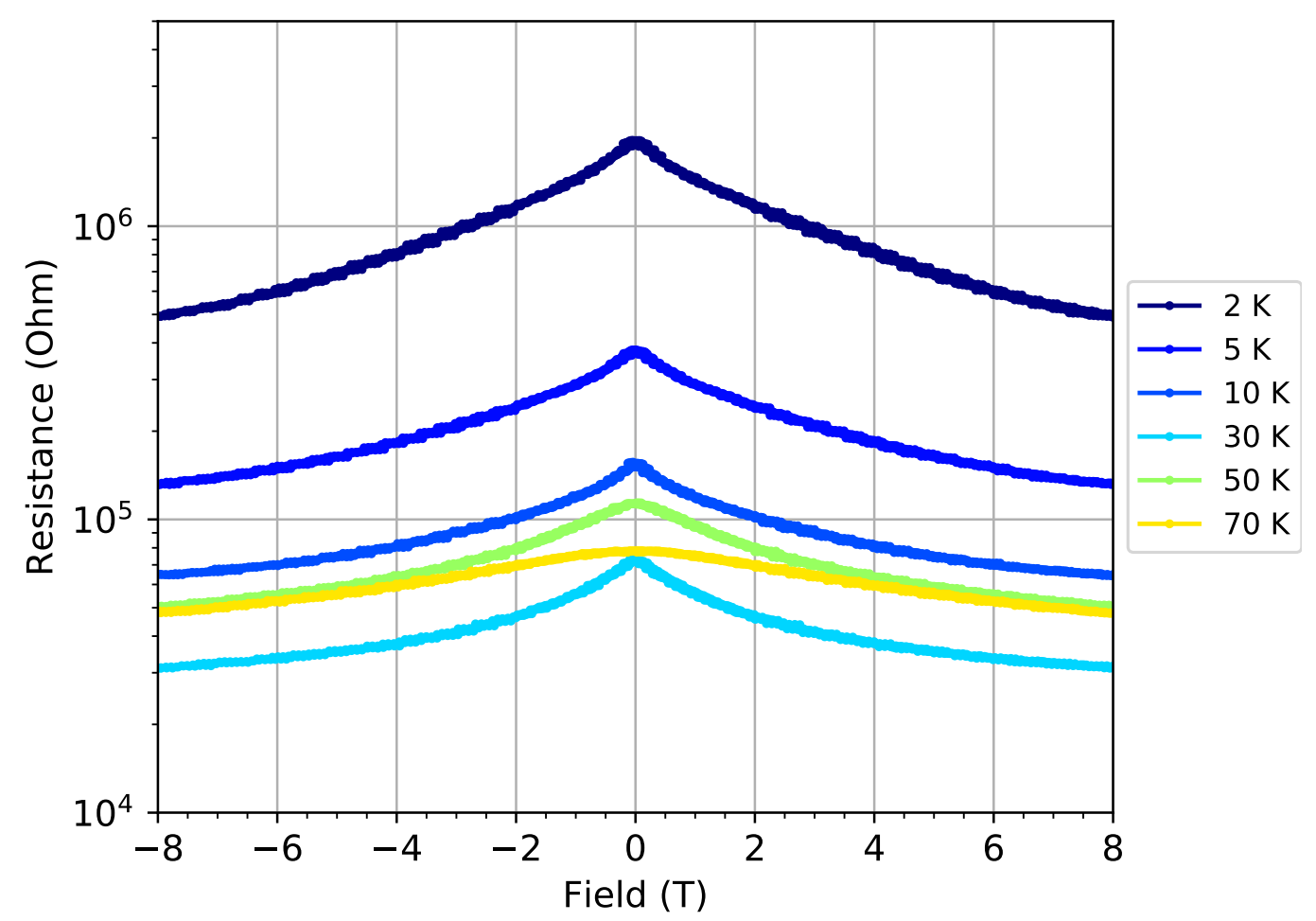

Figure 6.34: HR $(20 \mu \mathrm{m})$ : Log-plot of magnetoresistance at $10 \mathrm{nA}$ at different temperatures. 
To compare the magnetoresistance change, we plot the JMR for the same HR device in fig. 6.35. The magnetoresistance change for HR $(20 \mu \mathrm{m})$ is largest for the lowest temperature $(2 \mathrm{~K})$, in contrast to the LR devices.

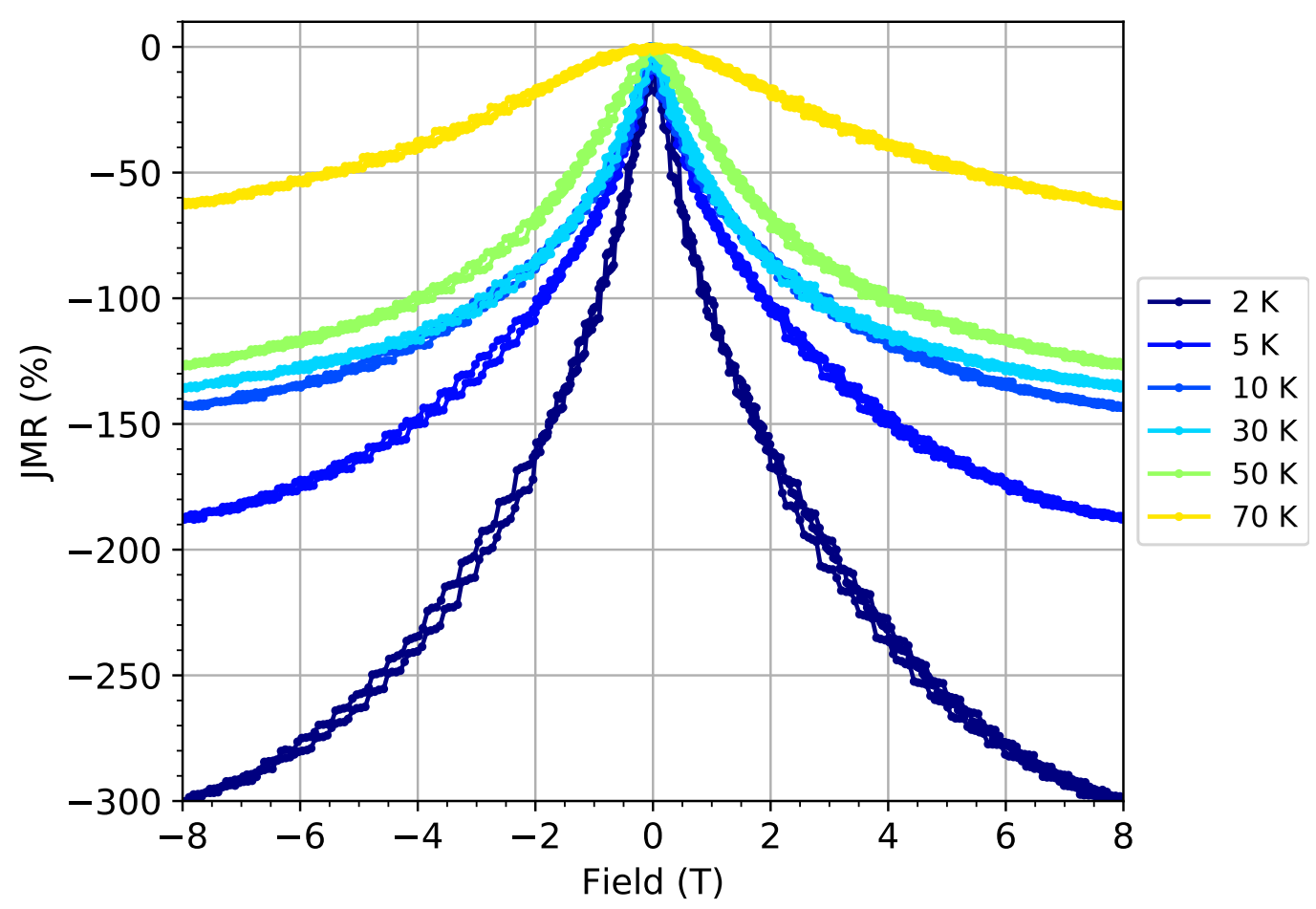

Figure 6.35: HR $(20 \mu \mathrm{m})$ : JMR at different temperatures. All are measured at $10 \mathrm{nA}$.

Neither HR nor LR devices show any additional structure in the JMR at large fields. The HR devices show a small hysteresis at low fields, shown in fig. 6.36. The hysteresis in the magnetoresistance is on the same magnitude as the coercive field of the GdN, suggesting that we see the GdN electrode change magnetisation direction. The $\mathrm{SmN}$ is not expected to switch easily as the coercive field is on the order of multiple Tesla. The magnitude of the hysteresis in the magnetoresistance in fig. 6.36 is similar for increasing and decreasing field at $2 \mathrm{~K}$ and $30 \mathrm{~K}$. The same hysteresis was not observed in LR devices. The coercive field of the LR devices is half of the HR devices, according to the magnetisation measurements above, 
suggesting that the hysteresis in the magnetoresistance was too small to observe accurately with the measurement setup (PPMS). Note that a new setup capable of measure large resistances and small fields is being built, with the instrument and sensor control code finished and included in appendix A, but still requires some work on the liquid helium cooling. This setup will aid further research.

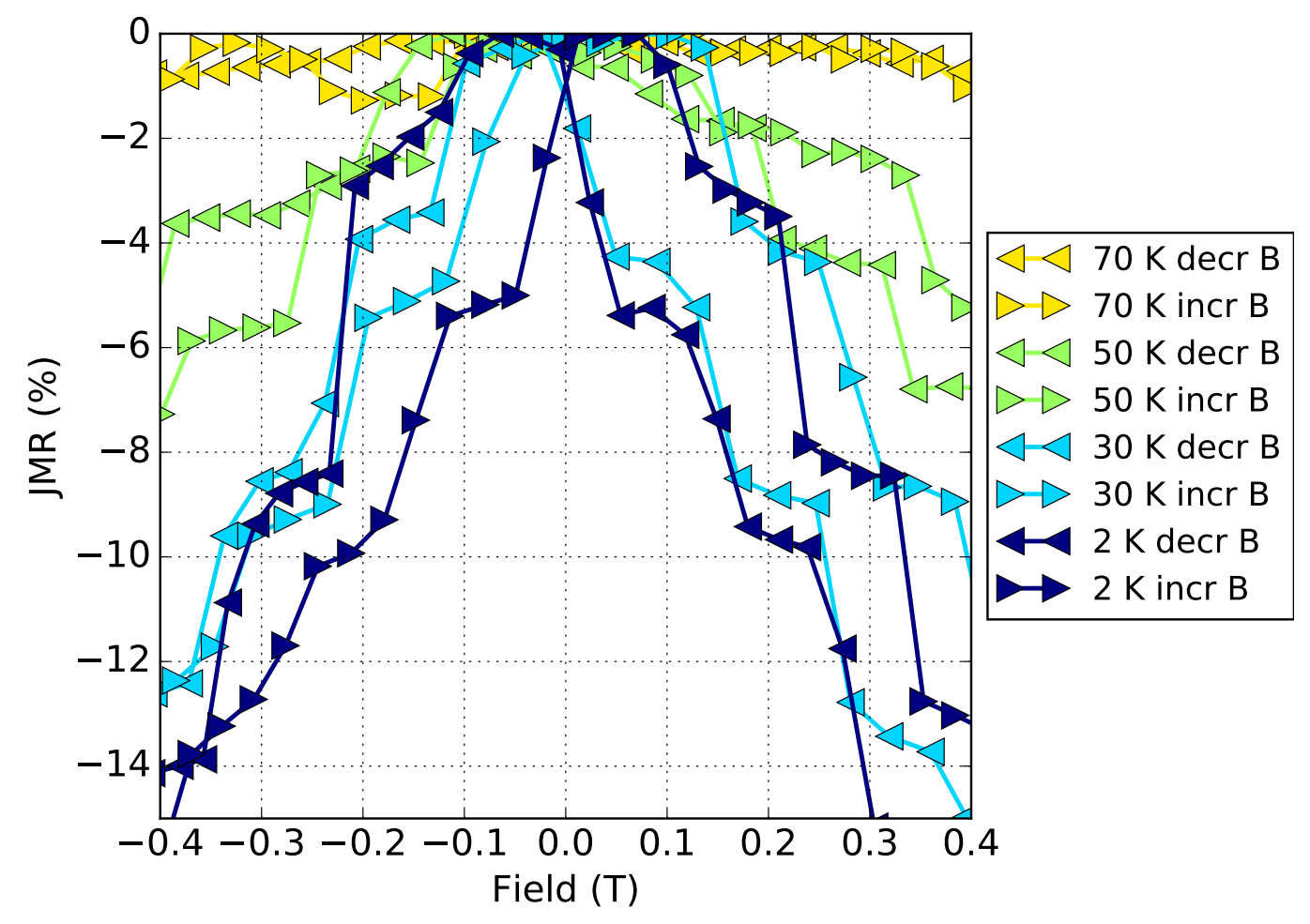

Figure 6.36: HR $(20 \mu \mathrm{m})$ : Magnification of low field JMR at different temperatures separated in increasing and decreasing field. All are measured at $10 \mathrm{nA}$.

The switching in fig. 6.36 persists up to $30 \mathrm{~K}$. At $50 \mathrm{~K}$, between $\pm 0.1 \mathrm{~T}$ in fig. 6.36, there is no hysteretic switching, but there is some difference in the up- and down-sweep of the field appearing as steps in the curves. The destruction of hysteresis above $30 \mathrm{~K}$ shows that the hysteresis requires both the GdN and SmN to be ferromagnetic. 
To extract more information about the high field behaviour and further compare the LR devices and HR devices we plot the TMR as a function of temperature for one LR device, and two HR devices in fig. 6.37.

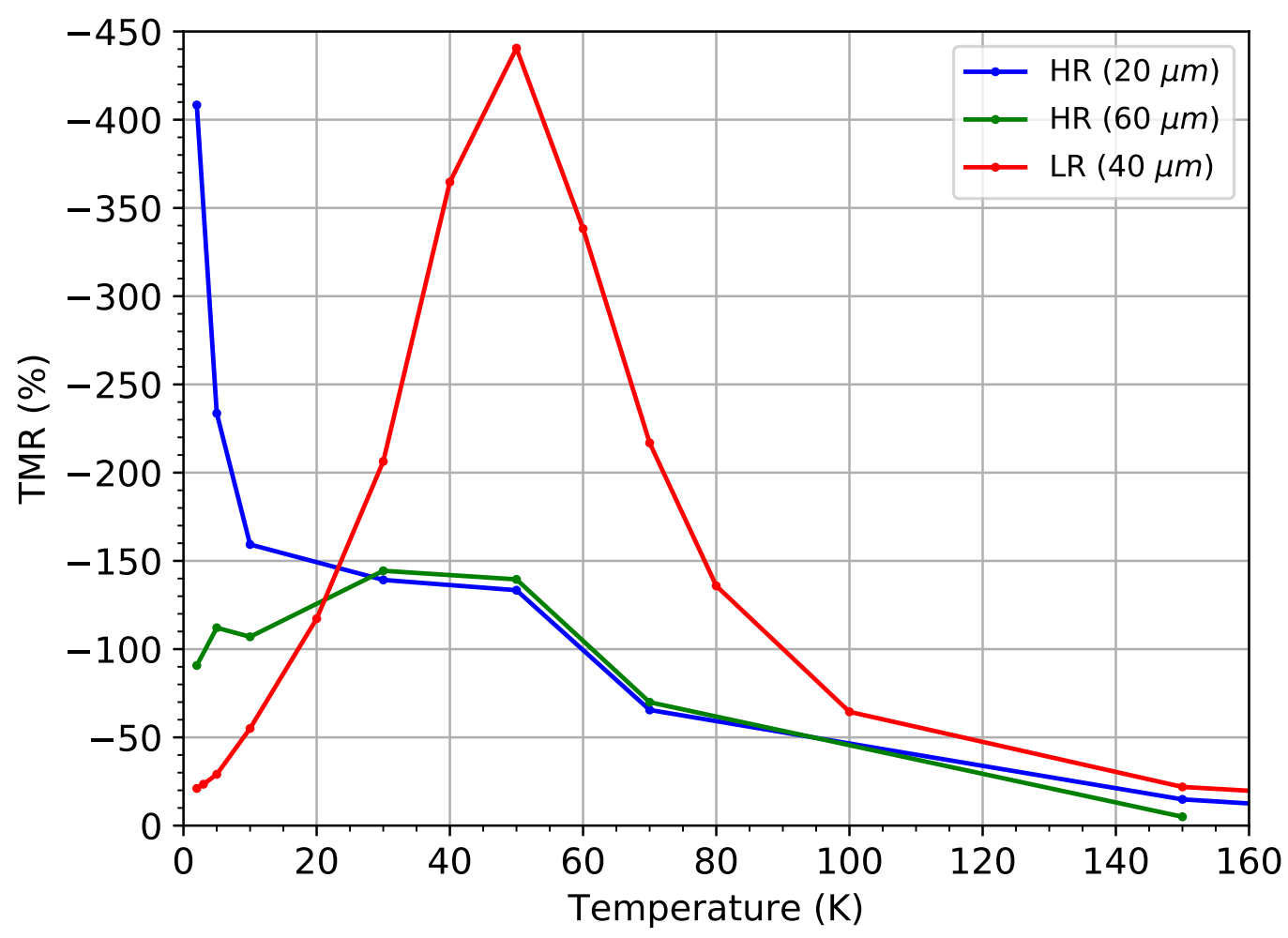

Figure 6.37: TMR at different temperatures for two HR devices and one LR device showing TMR.

In fig. 6.37 the LR $(40 \mu \mathrm{m})$ device has a maximum TMR at $50 \mathrm{~K}$. The HR $(60 \mu \mathrm{m})$ device also has a maximum TMR at $50 \mathrm{~K}$, while the HR $(20 \mu \mathrm{m})$ device only has a shoulder at $50 \mathrm{~K}$ and a maximum TMR at $2 \mathrm{~K}$, suggesting that the origin of the $50 \mathrm{~K}$ TMR exists in all devices, while the origin of $2 \mathrm{~K}$ TMR only exists in some HR devices. 
All the previous measurements have been done in the linear regime of the J-V curve. To investigate the JMR behaviour in different regimes of the J-V curves, we show fig. 6.38 where the field dependent JMR is shown for four currents through the LR $(40 \mu \mathrm{m})$ device. A current of $1 \mu \mathrm{A}$ corresponds to a measurement in the linear J-V regime. A current of $5 \mu \mathrm{A}$ corresponds to a measurement in the transition between the linear $\mathrm{J}-\mathrm{V}$ regime and the non-linear regime. The two larger currents are firmly in the non-linear regime. Figure 6.38 shows that the device behaves similarly regardless of the device being measured in the linear or non-linear $\mathrm{J}-\mathrm{V}$ regime. However, a lower current has a slimmer JMR profile with a sharper drop-off at low field than a higher current. The TMR is larger for lower currents.

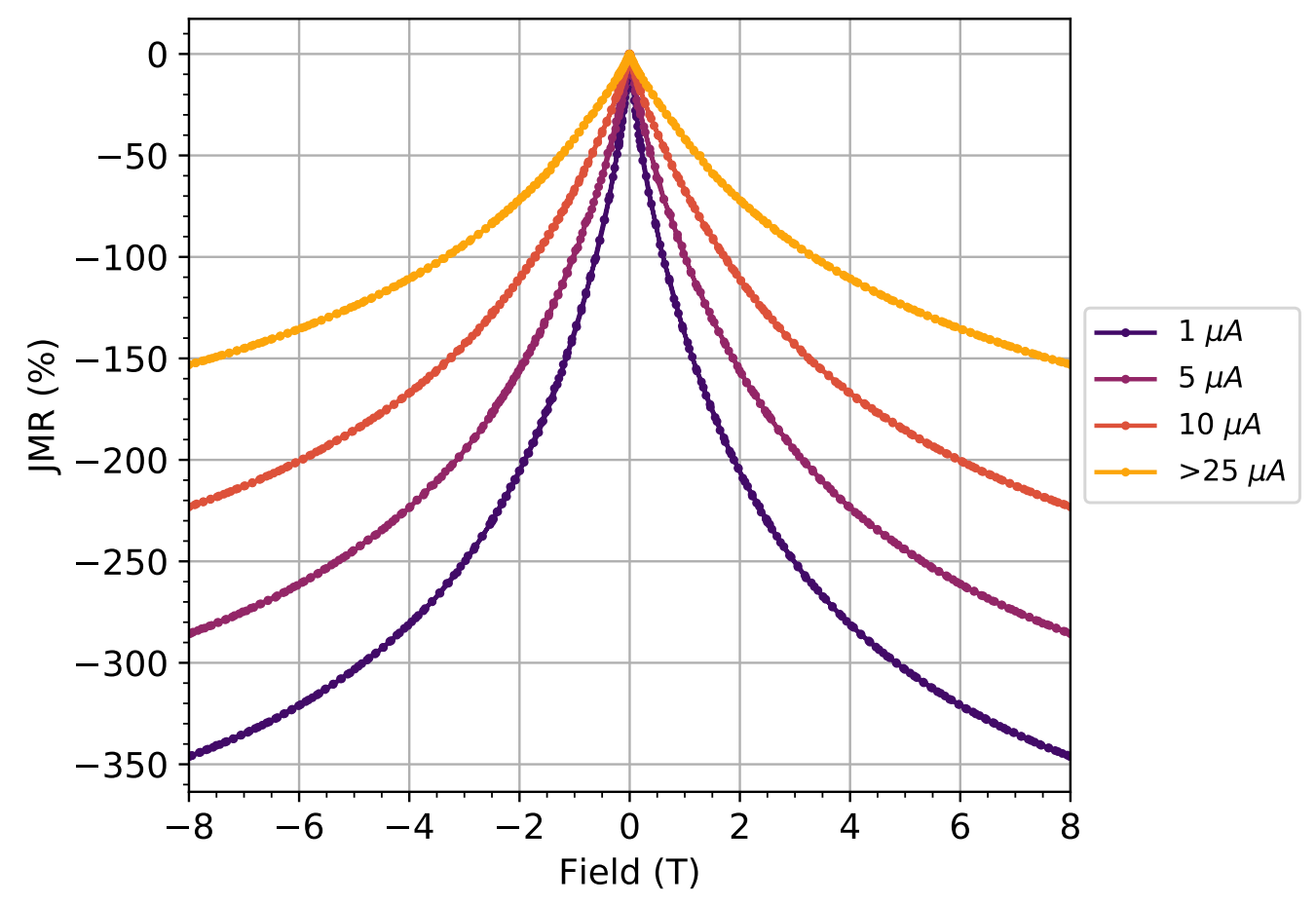

Figure 6.38: LR $(40 \mu \mathrm{m})$ TMR changes with different current at $60 \mathrm{~K}$. 
To investigate how the TMR changes through the changing J-V characteristics, we plot the TMR against the voltage across the junction for LR $(40 \mu \mathrm{m})$ for different temperatures in fig. 6.39. The graph shows both positive voltages (triangles) and negative voltages (circles).

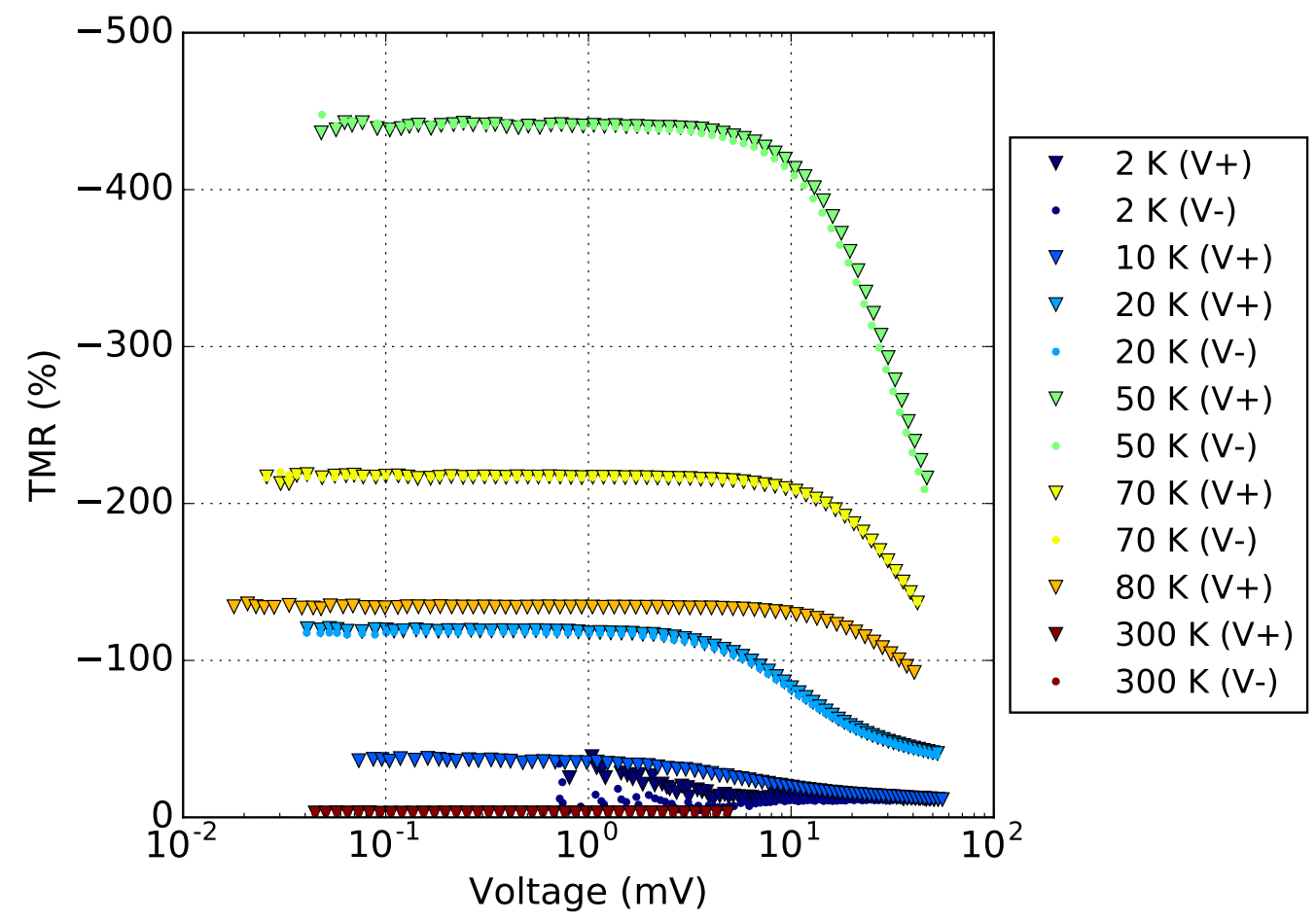

Figure 6.39: LR $(40 \mu \mathrm{m})$ : TMR dependence on voltage across the junction at different temperatures. Positive voltage (triangles), and negative voltage (circles) overlap.

For low voltages across the device, the TMR is constant, but as the voltage increases the TMR decreases. The TMR for temperatures at and above $70 \mathrm{~K}$ shows a parabolic drop-off while at lower temperatures there is a stronger bias-dependence in the $\mathrm{mV}$-region. It is interesting to note that even though the I-V characteristics of the LR devices are asymmetric, the voltage dependence of the TMR is symmetric.

Comparing the voltages at which the TMR drops in fig. 6.39 with the 
voltages at which the J-V curves become non-linear in fig. 6.27 there is a strong correlation between the start of the non-linear regime in the J$\mathrm{V}$ curves and the start of the drop in TMR. Investigating higher voltages would be of interest to understand if the TMR change continues to be symmetric at higher voltages and if the TMR drops to zero at sufficiently high voltage. Unfortunately, this could not be investigated due to voltage limitations of the experimental setup.

Most of the magnetic measurements performed in LR devices were, unless otherwise stated, performed at $1 \mu \mathrm{A}<1 \mathrm{mV}$ which is well in the constant TMR region for all temperatures. This means that even though the magnetoresistance measurements above were done at constant current, rather than constant voltage, the changes in JMR do not arise from a voltage change.

Figure 6.40, and fig. 6.41 shows how the TMR changes with the voltage across the junction at $2 \mathrm{~K}$ for two different HR devices: HR $(60 \mu \mathrm{m})$ with $50 \mathrm{~K}$ maximum TMR, and HR $(40 \mu \mathrm{m})$ with $2 \mathrm{~K}$ maximum TMR.

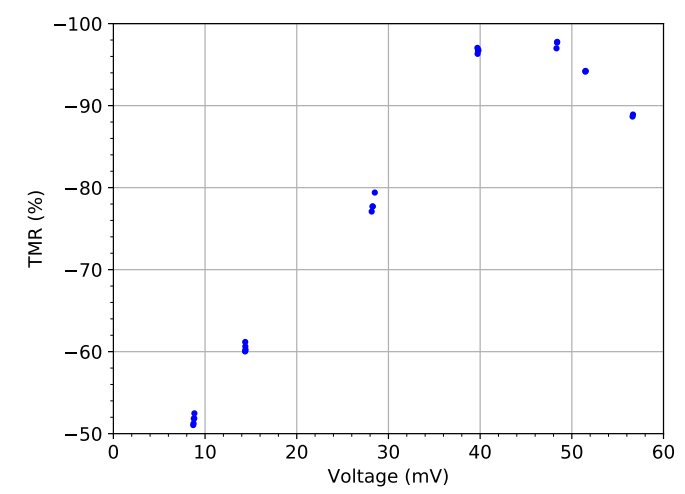

Figure 6.40: HR $(60 \mu \mathrm{m})$. This graph shows how the TMR changes with voltage across the junction at $2 \mathrm{~K}$ for the HR device where the TMR peaks at $50 \mathrm{~K}$.

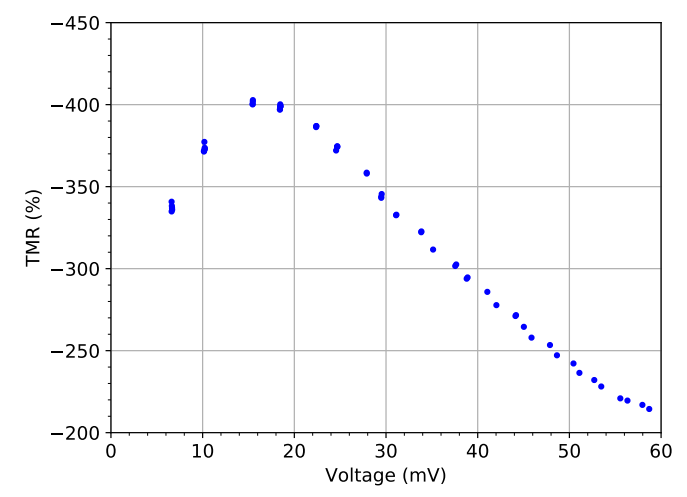

Figure 6.41: HR $(20 \mu \mathrm{m})$. This graph shows how the TMR changes with voltage across the junction at $2 \mathrm{~K}$ for the HR device where the TMR peaks at $2 \mathrm{~K}$.

The HR devices in fig. 6.40 and fig. 6.41 do not show the same be- 
haviour as the LR devices in fig. 6.39 with monotonically decreasing TMR with increasing voltage, but rather the HR devices show a peak in the TMR not symmetric around zero bias. The TMR peak occurs at different voltages in different devices.

All magnetic measurements above have been performed with the field in-plane. To motivate this choice, we compare the device resistance of HR $(20 \mu \mathrm{m})$ measured with the field applied in-plane and out-of-plane in fig. 6.42. The high-field behaviour in fig. 6.42 is similar for both in-plane and out of plane measurements. The low field behaviour shows a hysteresis in-plane but none out-of-plane. For the in-plane field direction, the magnetoresistance drops faster than the out-of-plane field direction close to zero. The out-of-plane measurement has a much wider top, and show little to no hysteresis. The in-plane measurements show a comparably sharp top. This demonstrates that it is more difficult to align the two electrodes out-of-plane, as expected from the shape anisotropy in a thin film [34]. 


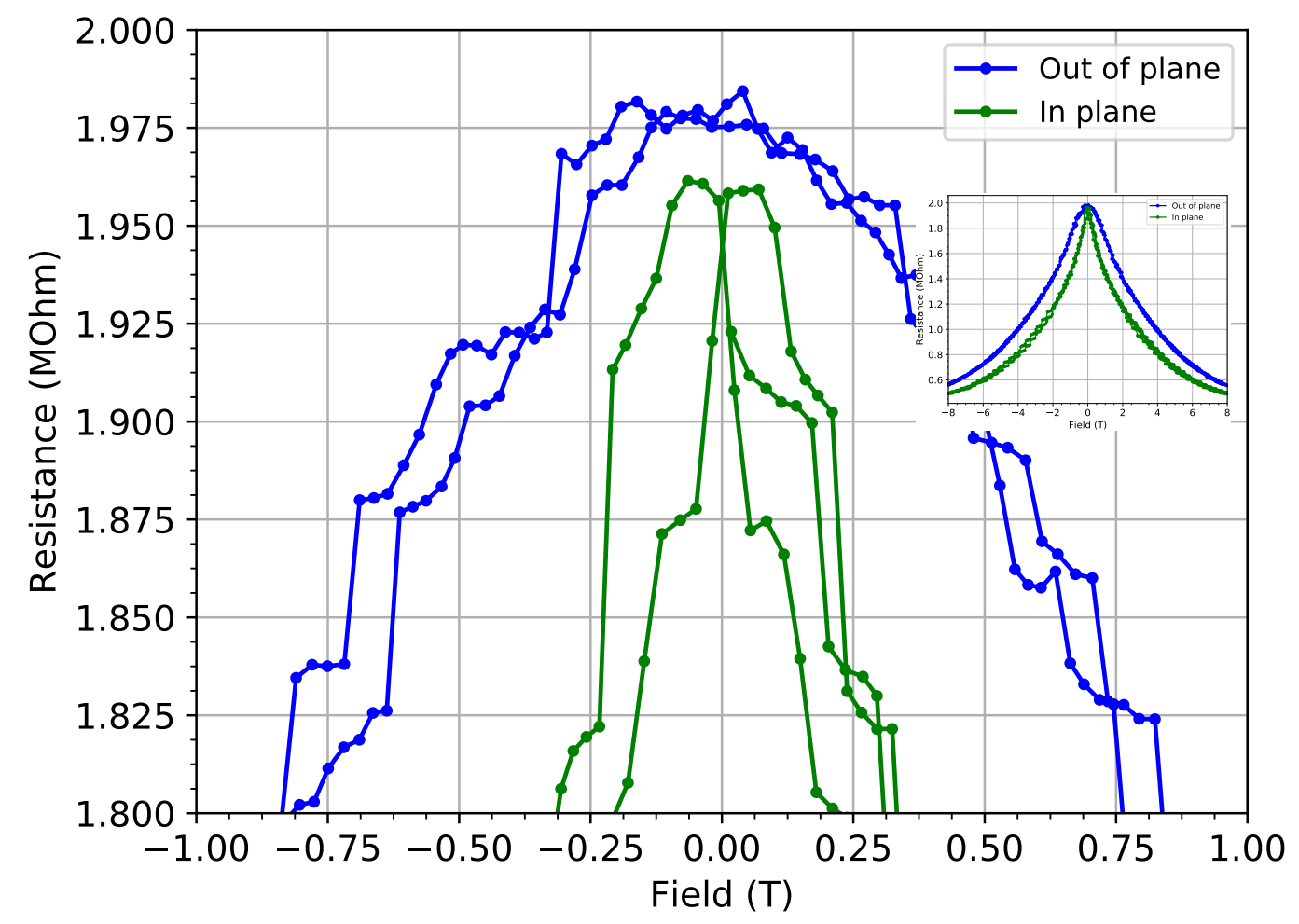

Figure 6.42: HR $(20 \mu \mathrm{m})$ Magnetoresistance measured with field out of plane and in plane (made directly after each other). Small hysteresis visible in IP but not OOP. Inset: full sweep between $\pm 8 \mathrm{~T}$. 


\subsubsection{Device breakdown}

Studying both working, breaking, and broken MTJs can teach us about the properties of the devices. We have spent the previous sections studying tunnelling devices, so in this section, we present two devices that broke and how the breaking changed the J-V characteristics.

In fig. 6.43 we see a J-V curve of a device which broke during the J-V measurement. The measurement starts at the lowest possible current for our cryostat $(10 \mathrm{nA})$ and increases the current in small steps (alternating positive and negative currents) while measuring the voltage. As the voltage across the junction reaches $2.1 \mathrm{~V}$ the behaviour of the device changes drastically. The next measurement point which uses a higher current again measures a resistance of the device which has dropped by four orders of magnitude (from $128 \mathrm{k} \Omega$ to $80 \Omega$ ). Subsequent J-V measurements of the device are linear. This demonstrates a failure mode of the device as going from a tunnelling regime to a conducting regime, likely from breaking the barrier.

The breakdown field of thick GaN in vertical transport orientation is theoretically calculated to be $3 \mathrm{MV} / \mathrm{cm}$ [109]. As the GaN layer for the device in fig. 6.43 is calculated to be $\sim 10 \mathrm{~nm}$ thick (from growth rate calibrations using SEM), and with an applied voltage of $2.1 \mathrm{~V}$ this corresponds to a field of $2.1 \mathrm{MV} / \mathrm{cm}$ across the tunnel junction. Different GaN MTJs manufactured for this thesis have shown varying breakdown fields, ranging from $1.9 \mathrm{MV} / \mathrm{cm}$ to above $16 \mathrm{MV} / \mathrm{cm}$.

Devices with thinner barriers did not reach the fields required for $\mathrm{GaN}$ breakdown. A thinner barrier results in a lower voltage for the same applied current. As the devices are strongly non-linear, a thinner barrier requires a much larger current than a thicker barrier to reach the breakdown voltage. At these higher currents the devices heated up by tens of degrees even when well thermally anchored to the cryostat cold finger. This meant no voltage breakdown could be observed in the cryostat. However, 


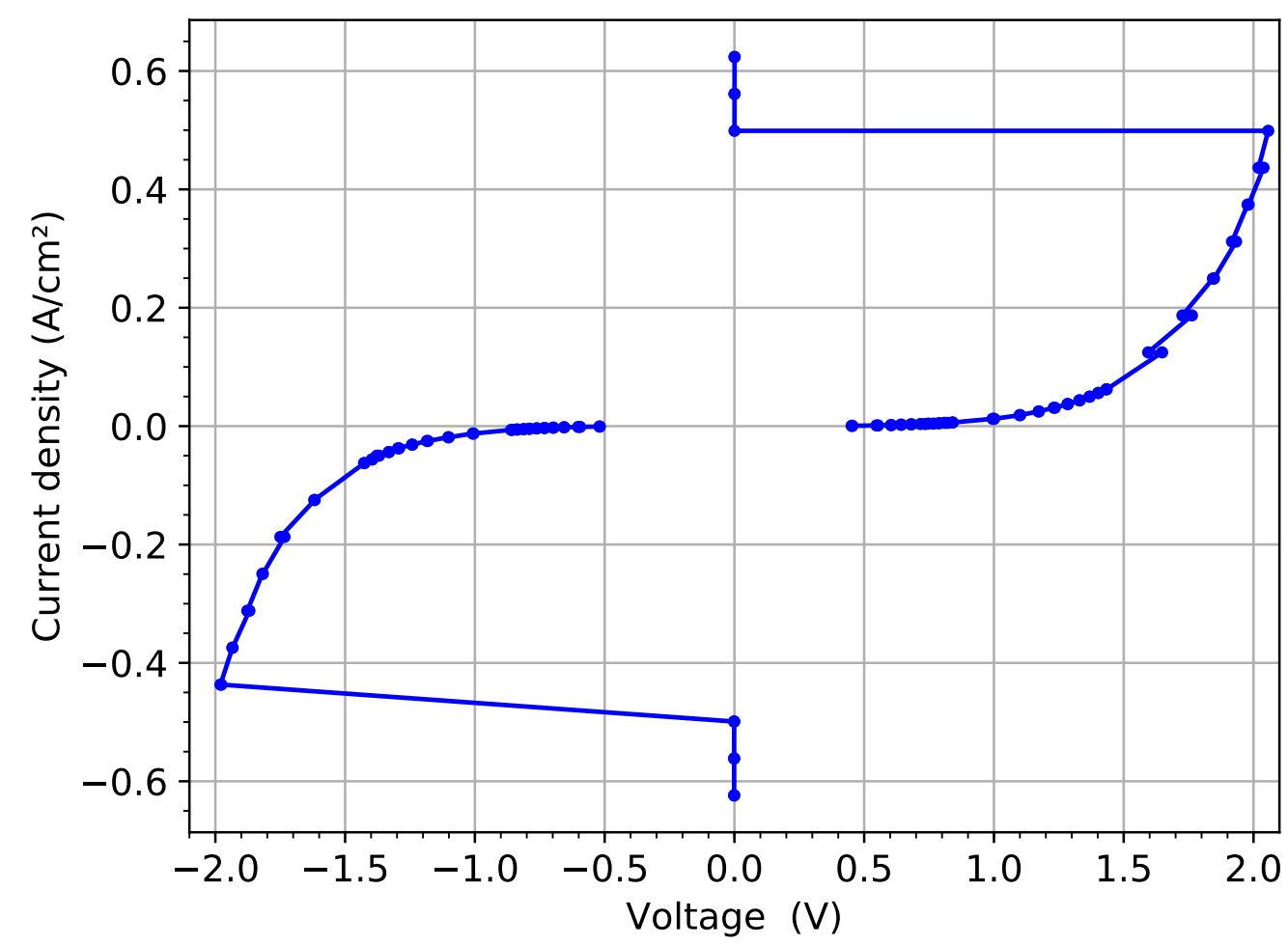

Figure 6.43: J-V curve from a single device at $4 \mathrm{~K}$ showing the breakdown of the barrier at $2.1 \mathrm{~V}$.

a number of devices with thin barriers were accidentally broken by using a hand-held multimeter to measure the resistance across the junction. The multimeter applies $9 \mathrm{~V}$ initially, which was enough to break the junctions whether from high voltage or high local temperature is uncertain. 
The effect of the breakdown on the J-V characteristics is illustrated in fig. 6.44 where the J-V curves of the HR device have been measured before and after a breakdown of the device are seen, with an applied field of $0 \mathrm{~T}$ and $8 \mathrm{~T}$. The resistance of the device decreases by almost four orders of magnitude when it breaks. The TMR of the device has changed from around $400 \%$ in the entire device to $3 \%$ in the broken device, lower than any of the working MTJs, demonstrating that the magnetoresistance measured in the working devices is controlled by the tunnelling through the barrier. 


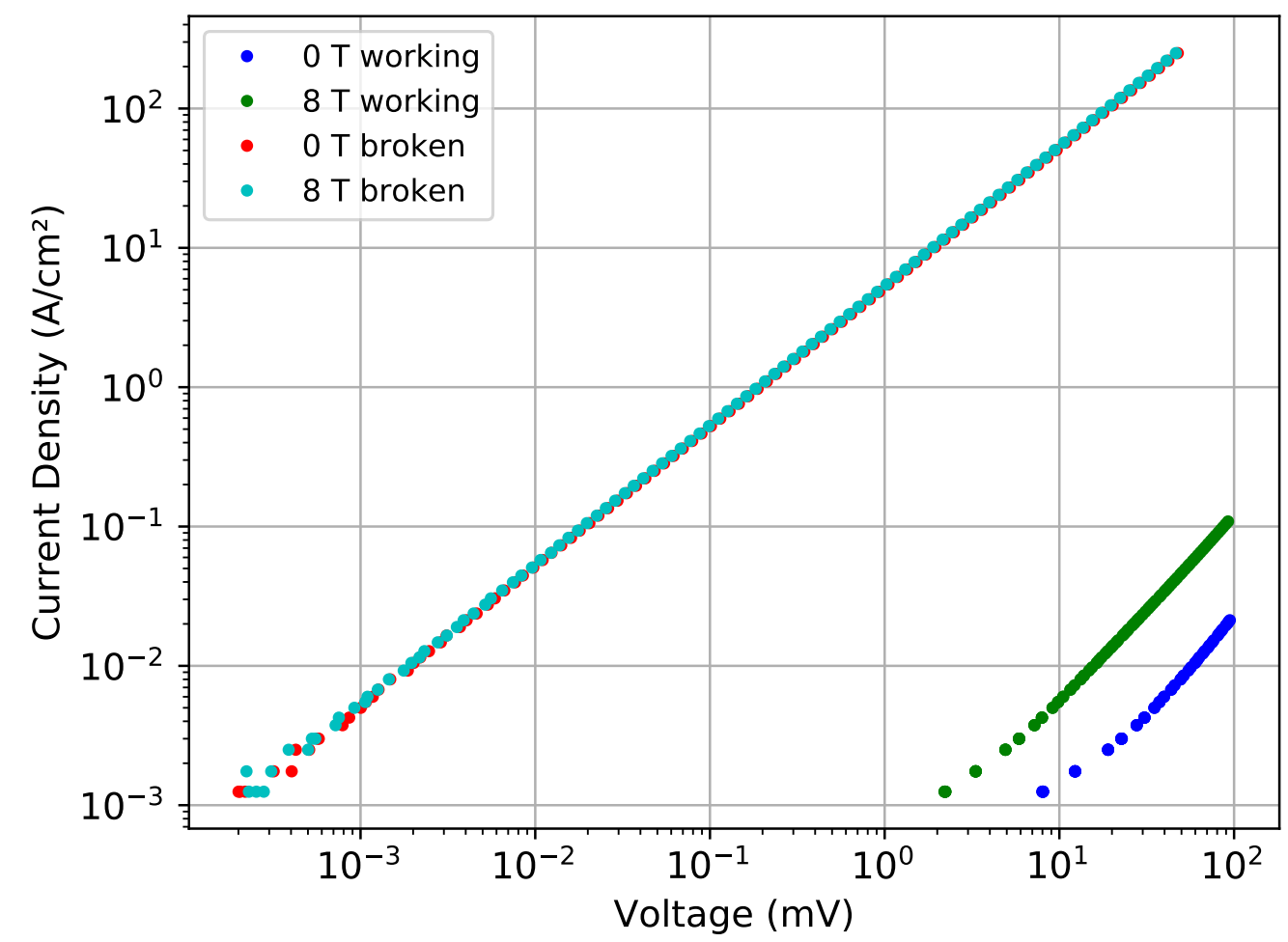

Figure 6.44: HR $(20 \mu \mathrm{m})$ pre and post-breakdown showing the J-V curves at $2 \mathrm{~K}$ shifting and becoming linear and the effect of the magnetic field diminishing when broken. 


\subsection{Discussion}

The MTJs in this chapter show a strong temperature and voltage dependence for the tunnel current and TMR. This strong dependence is not supported in the standard direct tunnelling model but could be explained simply by the spin-polarised density of states changing differently with temperature and voltage in the GdN and SmN. However, the MTJs in this chapter show a much stronger temperature dependence than the MTJs in Refs $[9,99]$ suggesting that the changing spin-polarised density of states is only part of the explanation of the strong temperature and voltage dependence.

As the SmN we grow is usually an orbital-dominant ferromagnet and the $\mathrm{GdN}$ is a spin-dominant ferromagnet, they should align opposite in a magnetic field. Opposite spin alignment should result in the high field state being anti-parallel (high resistance) and the low field state being parallel (low resistance), i.e. positive TMR [37,99]. However, all MTJs in this thesis show strong negative TMR suggesting that the $\mathrm{SmN}$ electrode has the same spin-alignment as the GdN electrode. This is further supported by the switching from a high resistance state to a low resistance state when the GdN switches magnetisation direction.

A diagram of the data from fig. 6.36 together with the electrode spin alignment is shown in fig. 6.45. The bottom diagram shows the resistance of the MTJ when the field is swept either up (orange) or down (blue), together with the average resistance of the two directions (light blue). The top diagram shows how the resistance of the MTJ changes when subtracting the large average resistance. At low field, we see the GdN switch after passing zero changing the MTJ from a low resistance state (parallel) to a high resistance state (anti-parallel). The high field behaviour of switching the $\mathrm{SmN}$ to reach a low resistance state cannot be distinguished from the large negative JMR, but as the GdN switches in both directions, we assume that the SmN switching occurs but is hidden by the large JMR. 


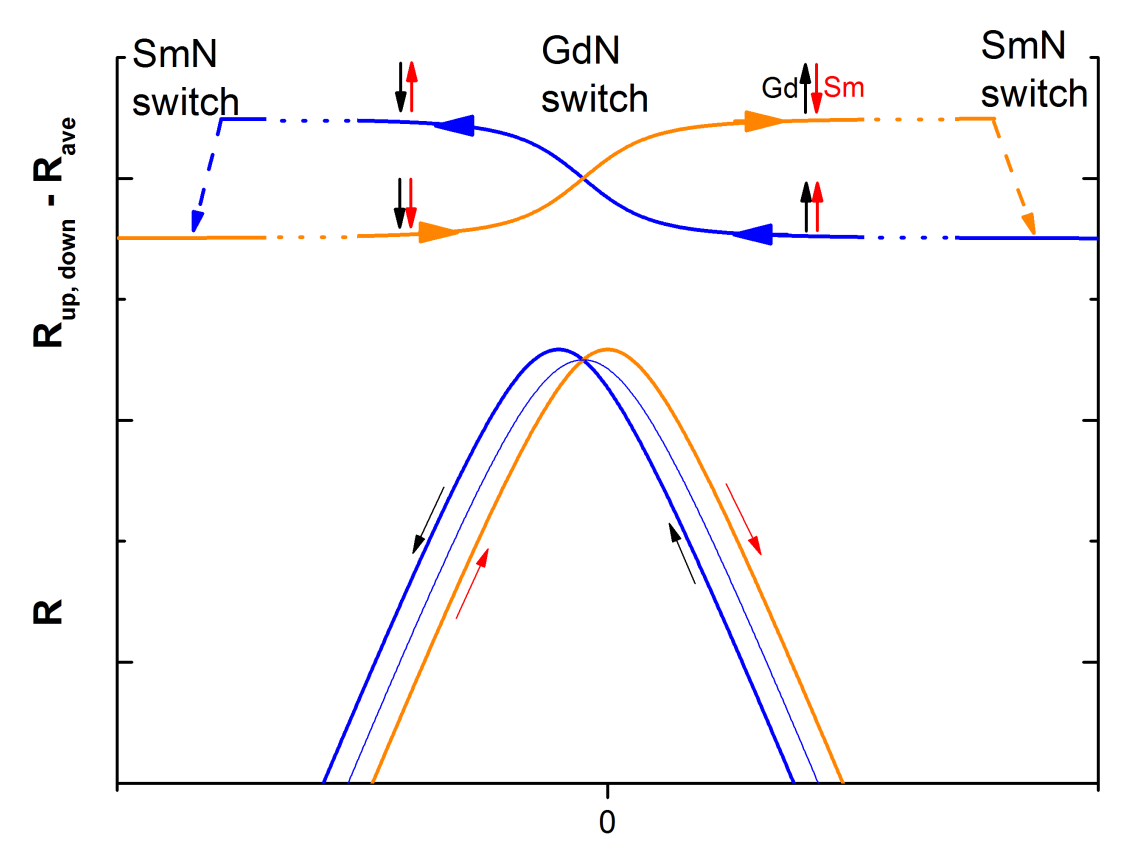

B

Figure 6.45: Bottom: A diagram of the resistance during the switching of the MTJ. Top: A diagram of the difference between average resistance and the two sweep directions together with the magnetic orientation of the electrodes.

Growing spin-dominant $\mathrm{SmN}$ is possible if the doping is sufficiently high, $\sim 3 \%$ giving a carrier concentration of $\sim 10^{21} \mathrm{e}^{-} / \mathrm{cm}^{3}$. Such a high doping level forces electrons to start filling the $5 \mathrm{~d}$ band which gives the additional electrons majority spin and shifts the balance from orbital-dominant to spin-dominant. As was seen in fig. 5.12 it is possible to grow $\mathrm{SmN}$ that has been doped to degeneracy. However, the $\mathrm{SmN}$ in the presented MTJs was grown under conditions which have produced orbital-dominant $\mathrm{SmN}$ in the past.

It is also possible that the $\mathrm{SmN}$ is being spin-flipped by spin-transfer torque. The device dimensions in this thesis are much larger, and hence the current density much smaller, than in other systems where this is observed 
[110]. However, if the tunnelling transport was concentrated in hotspots or even pinholes, the local current density could be orders of magnitude larger than the average current density for a uniform barrier, potentially reaching the current densities required for spin-transfer torque to influence the MTJ. To reach a current density of $10^{10} \mathrm{~A} / \mathrm{m}^{2}$ required for spintransfer torque [110] all current in our devices would have to pass through an area of $<1 \mathrm{~nm}^{2}$ without causing any local temperature changes.

On top of the switching, there is large negative magnetoresistance which we attribute to the $\mathrm{GdN}$ as it persists above the $\mathrm{T}_{C}$ of $\mathrm{SmN}$ and does not change until above $\mathrm{T}_{C}$ of $\mathrm{GdN}$. To understand this, we look closer at the GdN layer. The JMR for both HR and LR device behave similarly despite the field-dependent magnetisation curves having different behaviour (see fig. 6.12 and fig. 6.14), suggesting that the bulk film magnetisation is not representative of what happens in the MTJ. The saturation magnetisation of the surface layers can be reduced significantly from the bulk value even without taking ion damage into account [90]. As the tunnel electrons originate from the top 1-2 monolayers [93,96], the magnetisation of the GdN surface, not the bulk film, will be determining the tunnel properties. Looking back at the experiment with ion-exposed GdN in section 5.3, the expanded phase GdN (which gives rise to the non-saturating magnetisation $\mathrm{GdN}$ ) exist in the surface of the GdN film which had only been exposed to ions through a protective $\mathrm{Al}$ film (see XRD data in fig. 5.7). When the $\mathrm{GaN}$ tunnel barrier is deposited on the GdN electrode, the $\mathrm{GdN}$ is exposed to ions which will turn the surface layers into the expanded phase GdN. Indeed the HR sample (which has been exposed to ions for longer) has a magnetisation curve which behaves more like the ion exposed GdN. The magnetisation of the GdN layers which participate in the tunnelling can then likely be described similarly to the ion damaged GdN in fig. 5.8. This suggests that the monotonic JMR change at large field arises from the GdN electrode slowly reaching saturation, rather than sharply saturating.

With the GdN surface being changed by the nitrogen ion exposure dur- 
ing the tunnel barrier growth it is possible that not only the structural and magnetic properties change, but also the electrical properties. The measurements of the resistivity of the ion-exposed GdN in section 5.3 suggest that the ions make the GdN more insulating, particularly at low temperature. If the GdN surface layers behave like an insulator, the total barrier thickness of the MTJ consists of both the GaN and the insulating GdN. $\mathrm{GdN}$, as prepared by sputtering, has been used as an insulating barrier in tunnel junctions [7] showing that our surface GdN layers, if insulating, can behave as a barrier.

This leads us to suggest that the MTJ layers may differ from the intended design, seen in fig. 6.46, with a double barrier of $\mathrm{GaN}$ and $\mathrm{GdN}$. The change from the bulk GdN to the expanded surface GdN is likely gradual, giving a poor quality 'interface'. The nanocrystalline structure of the $\mathrm{GaN}$ also ensures rough interfaces with both the $\mathrm{GdN}$ and $\mathrm{SmN}$.

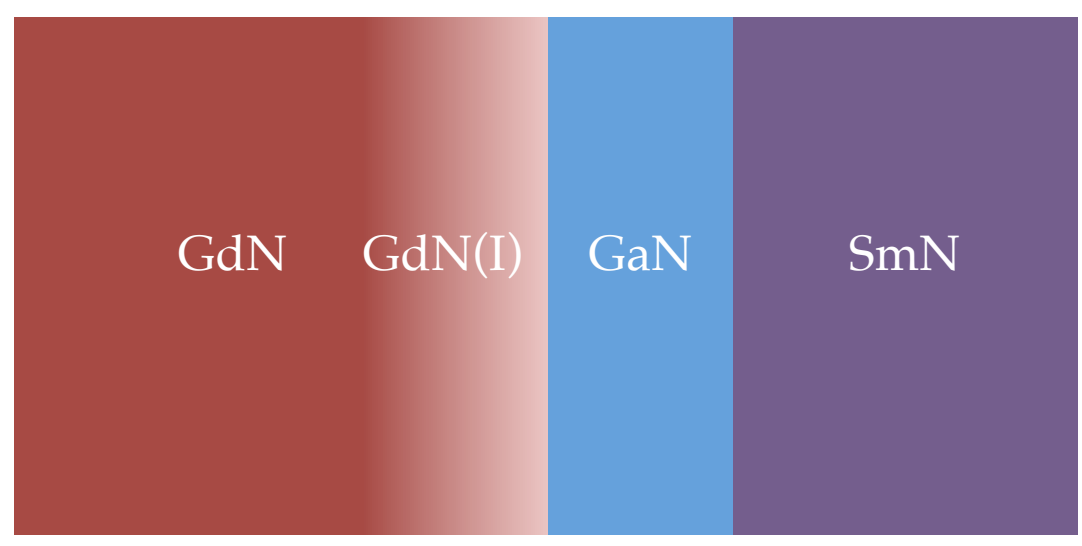

Figure 6.46: Suggested actual MTJ layer composition. GdN, insulating GdN, GaN, and SmN.

Ref [86] show that in ferrimagnetic tunnel barriers the tunnelling is dominated by scattering and spin waves (magnon assisted tunnelling) in the surface in the paramagnetic phase. Below $\mathrm{T}_{C}$ the tunnelling becomes dominated by spin waves inside the barrier. When the tunnelling is dominated by surface effects, the TMR is asymmetric with bias, and when the 
tunnelling is dominated by spin waves in the barrier, the TMR becomes symmetric with bias [86]. In MTJs with non-magnetic barriers asymmetry in the bias dependence of the TMR have been found to arise from electrodes of different materials $[88,96]$.

The shape of the bias dependence of the TMR below $70 \mathrm{~K}$, with the rapid drop in the $\mathrm{mV}$-range is indicative of magnon-assisted tunnelling in our devices. At and above $70 \mathrm{~K}$ the TMR drops parabolically with bias suggesting the tunnelling is no longer magnon assisted. In our LR MTJs, the TMR is symmetric with voltage below $70 \mathrm{~K}$. In contrast, the TMR-V curves of the HR devices are all asymmetric even at $2 \mathrm{~K}$, with the amount of asymmetry differing between devices, suggesting different interface effects further complicated by the possibility of pinholes in the HR devices.

The barrier height as extracted from the Simmons fit is well below the expected value at all temperatures, suggesting two-step tunnelling via defects. These defects can be located around the circumference of the active area, as suggested by the excellent device-to-device scaling of the I-V characteristics with the circumference. The distribution and composition of the defects in the barrier will influence the temperature and voltage dependence. Ref [91] find that the bias at which the nonlinearity in the I-V characteristics sets in, correlates with the onset of the bias dependent drop in the TMR. This is caused by two-step tunnelling via thermally generated defects. Thermally generated defects are unpolarized, which means the tunnel current via these defects does not contribute to the TMR. If the defects are uniformly distributed in energy, the density of defect states will rise with temperature and voltage [91]. We may also have two-step tunnelling via defects bands in the barrier giving rise to resonant tunnelling [83].

The barrier thickness has a strong influence on all forms of tunnelling. As the barrier gets thicker and the device resistance increases the fraction of the tunnel current that goes via the two-step tunnelling increases which decreases the TMR [92]. This can potentially explain why the TMR 
of the HR devices (above $20 \mathrm{~K}$ ) is smaller than the TMR of the LR devices. If the devices have pinholes, then transport through the pinholes and hotspots will dominate at low temperature [81]. Different kinds of pinholes or hotspots in different devices can explain the variation in TMR behaviour below $20 \mathrm{~K}$ in the HR devices.

The TMR of the MTJ devices in this thesis where the devices switch is a couple of percent, which is low compared to current state of the art MTJs of hundreds of percent. This difference is expected as our devices are polycrystalline, which have much lower TMR than epitaxial MTJs [111]. In fact, the TMR of our devices are comparable to other polycrystalline devices $[73,93,111]$ which have a TMR for switching in the range of $1 \%$ to $20 \%$. The overall JMR of our devices is high thanks to the large magnetoresistance of $\mathrm{GdN}$, promising a large TMR for switching if the barrier and electrode crystal quality can be improved, perhaps by epitaxial growth.

\subsection{Conclusions and future work}

We have successfully manufactured GdN/GaN/SmN MTJs with consistent behaviours between devices from the same growth, and studied how the MTJs behave when changing the active area of the device. From the more consistent MTJ manufacturing we have drawn a number of conclusions for future work.

The electrical and magnetic behaviour of GdN/GaN/SmN MTJs has been thoroughly investigated, with a particular focus on two sets of devices: High resistance devices (HR) and low resistance devices (LR). All devices show strong temperature and voltage dependence on current and TMR. The HR devices show clear switching of the GdN at the magnetic field corresponding to the coercive field of the GdN measured in the magnetisation. The GdN in the LR devices has a smaller coercive field, and the switching could not be clearly resolved using the available equipment.

Most strikingly we find the $\mathrm{SmN}$ to align its spin with the magnetic 
field rather than antiparallel. We suggest this arises from either high doping of the SmN skewing the spin and orbital contributions by doping into the $5 \mathrm{~d}$ band, or from spin-transfer torque from high current densities in hotspots in the barrier.

The decrease in TMR with applied voltage and the poor fit to the Simmons model indicates contributions from both defect-assisted tunnelling and spin waves in the barrier or barrier interfaces. To understand the behaviour of these devices in more details, the next step would be to conduct inelastic electron tunnelling spectroscopy (IETS). IETS can distinguish between different kinds of inelastic tunnelling and may help us understand which contributions occur under different conditions.

To move towards GdN/I/SmN devices with positive TMR, like in Refs $[9,99]$, we suggest that attention is paid to the barrier quality and the barrier interfaces. As the barrier quality improves, the elastic (direct) tunnelling fraction will increase which will increase the TMR. A higher quality barrier also gives rise to sharper switching. To achieve higher quality barriers in polycrystalline junctions the barrier material has to be chosen and grown carefully. A polycrystalline barrier will always smear the switching, but the degree of switching may improve as the crystallinity of the barrier increases.

To achieve sharper switching of the GdN the magnetisation in the surface layers needs to be able to switch and saturate sharply. As this has been shown to be influenced by the ion source, the growth conditions for the barrier need to be controlled not only for the sake of the barrier but for the quality of the GdN.

While the MTJ design using photolithography which was developed for and presented in this chapter and chapter 3 has shown some advantages over previous designs, we highlight considerations for future work with photolithography based MTJ. The new design allows us to study device consistency across 20 devices on a 2 inch wafer which is an order of magnitude improvement over earlier designs. The area restriction layer 
was added to the cross-contact geometry to allow smaller devices to be fabricated more easily.

The area restriction layer introduces additional edges around the circumference where the tunnel stack has to bend to accommodate the change in geometry. It is clear that this edge effect influences the devices, but it is not clear whether the devices will perform better with a very sharp edge (which gives a well-defined device size) or with a more gently curved edge (which lets the tunnel stack transition less abruptly between the bottom contact and the area restriction layer). To understand the effect of the edge of the active area, one would need to change and optimise the photolithography patterning, including possibly changing the photoresist.

When using an area restriction layer, the resistance of the MTJ needs to be small compared to the resistance of the area restriction layer as otherwise the restriction layer does not actually restrict the area, but provides a parallel current path. This can be achieved by only making lower resistance devices, using a more resistive material in area restriction layer, or even growing thicker area restriction layers. AlN would be an excellent choice for a more resistive material, but unfortunately, all photoresist developers tested dissolve the AlN completely removing it when patterning the stack layer. Working towards smaller devices using photolithography necessitates the use of an area restriction layer due to the pattern resolutions possible with the available equipment.

To achieve very high TMR and sharp switching we need to move to epitaxial devices, which do not use photoresist for the deposition of the REN electrodes and barrier as the temperatures required for epitaxial growth are too high for photoresist. The design and development work I put in towards making shadow-masked MTJ stack structures has made a start towards this goal. Making epitaxial devices, in the current device design, would also require the bottom contact to be epitaxially compatible with the bottom electrode as well as being metallic. One possible option is $\mathrm{HfN}$, non-magnetic and metallic with a $\mathrm{NaCl}$ structure a close lattice 
match to many of the RENs [1]. The barrier layer would need to be epitaxially compatible with both electrode materials as well as insulating. One suggested epitaxially compatible barrier material is $\mathrm{LaN}$, which is insulating and non-magnetic [1].

An option to using shadow-masking for epitaxial growth is to use focused ion beam milling or electron beam lithography to essentially cut out devices from a large sheet of unpatterned layered materials. A worry here is that this can be destructive to film quality [112], and given the influence of ions on GdN described in section 5.3, devices patterned in this fashion could behave very differently to devices patterned without ions.

At the fundamental research end of the MTJ improvement, a lot of material properties of the materials in the REN MTJs are not yet explored and would add predictive and explaining power to the behaviour of the MTJs. Knowing properties of the RENs such as the dielectric constant, interface and film quality and structure in multilayers, and especially experimental measurements of the band structure and how it changes with temperature and applied voltage would guide theoretical efforts.

While no complete model can be pinned down for the behaviour of the MTJs in this chapter, we provide a sizeable experimental basis for further future theoretical investigations. All effects discussed as possibilities for the behaviour of the MTJ, such as defect- and magnon-assisted tunnelling, are known to severely decrease the TMR of MTJs, suggesting that we have barely scratched the surface of the making devices with exceedingly strong responses to magnetic fields. 


\section{Chapter 7}

\section{Summary and Outlook}

\subsection{Results}

This thesis has made a number of progress steps in the fabrication and understanding of rare earth nitride devices. This thesis has gone from investigating the reaction that forms the rare earth nitrides, to studying properties of the rare earth nitrides, to the creation and investigation of rare earth nitride magnetic tunnel junctions.

We showed conductance measurements during the catalytic cracking of $\mathrm{N}_{2}$ and the subsequent nitriding in three rare earths. We identified an initial rapid nitridation of the top $1.5 \mathrm{~nm}$ before the $\mathrm{N}_{2}$ pressure even stabilised. Following the nitridation of the top $1.5 \mathrm{~nm}$ we identified a transition from a reaction limited regime to a diffusion limited regime based on a pause in the conductance drop. In the high nitriding limit, the conductance change corresponds to between $18 \%$ to $50 \%$ of the RE film having converted to a REN. Films of Sm, Gd, and Dy showed similar nitridation characteristics.

From the nitridation experiments, we proposed an upper bound on the growth rate of $S m N$ grown by co-deposition of $S m$ and $N_{2}$ of $400 \mathrm{~nm} / \mathrm{h}$, based on the rate of initial rapid nitridation of Sm. If a catalytically active 
$\mathrm{RE}$ is deposited faster than $400 \mathrm{~nm} / \mathrm{h}$ in a $\mathrm{N}_{2}$ atmosphere the resulting film will likely be, unintendedly RE rich.

We showed that the conductivity of $\mathrm{SmN}$ is inversely proportional to the $\mathrm{N}_{2}$ pressure during growth, displaying the same behaviour as the already published results in GdN. This suggests that the mechanism for doping the two materials is the same, strengthening the argument that both $\mathrm{SmN}$ and $\mathrm{GdN}$ are doped by nitrogen vacancies in the lattice. The dopability of both $\mathrm{GdN}$ and $\mathrm{SmN}$ is useful for tuning device properties, such as impedance matching electrical components for different applications.

We showed that the nitrogen atmosphere post-growth of SmN influences the conductivity of the SmN. The nitrogen in the surface layers of a polycrystalline $\mathrm{SmN}$ thin film appears to be more weakly bound. By leaving the as-grown $\mathrm{SmN}$ film in $1 \cdot 10^{-8} \mathrm{mbar}$ for a few minutes the nitrogen escapes the top $4 \mathrm{~nm}$ leaving a more conductive layer. However, the nitrogen vacancies in the surface layers can be filled as rapidly as they are created by introducing $\mathrm{N}_{2}$ at the growth pressure into the chamber. We also presented the first-ever estimate of the nitrogen equilibrium pressure in $\mathrm{SmN}$ giving the value of $5 \cdot 10^{-6}$ mbar to $3.5 \cdot 10^{-5}$ mbar.

We show that the nitrogen ions used to fabricate capping layers of AlN or GaN on top of the RENs can change the REN film. GdN exposed to ions during the growth of AlN shows a $1.6 \%$ expanded lattice parameter, as compared to an identical GdN film not exposed to ions during the growth of $\mathrm{Al}$ as a capping layer. The ion exposure lowered the magnetic moment of the GdN film and pushed the field at which the magnetisation saturates to above $7 \mathrm{~T}$. A second magnetic transition arose at $50 \mathrm{~K}$ in the ion exposed GdN which was not visible in the Al capped GdN. The low-temperature resistivity was two orders of magnitude larger in the ion exposed GdN. These results suggest that great care must be taken both when comparing RENs fabricated by different methods and capped with different materials. It also means that when manufacturing tunnel devices 
using AlN or GaN as a barrier the REN film below the tunnel barrier will be affected by the nitrogen ions.

With all of these considerations in mind, we fabricated magnetic tunnel junctions under well-controlled conditions. We demonstrated a new design which allowed 20 devices to be manufactured simultaneously with precisely known active area dimensions. The new device design is insensitive to $0.75 \mathrm{~mm}$ misalignment of the final step which allowed us to develop and use shadow masking. Within a growth of 20 devices, the electrical characteristics were consistent for low resistance devices, suggesting that the new device design and growth is consistent across the substrate. High resistance devices showed a parallel path through the area restriction layer due to the comparable resistance of the device and the GaN layer, so we suggested future design improvements for high resistance devices.

$\mathrm{J}-\mathrm{V}$ characteristics of MTJs were measured over seven orders of magnitude in current density, up to $4000 \mathrm{~A} / \mathrm{cm}^{2}$, and five orders of magnitude in voltage, up to $5 \mathrm{~V}$. All devices showed asymmetric non-linear J-V characteristics which changed with temperature.

When a device was broken by applying a too large voltage, the J-V characteristics became linear, and the resistance dropped by more than three orders of magnitude. Working devices showed a maximum magnetoresistance of $100 \%$ to $600 \%$, while broken devices showed a maximum magnetoresistance of $3 \%$, demonstrating that the magnetoresistance measured in the working devices is indeed controlled by the tunnelling through the barrier.

We demonstrated switching of the GdN electrode in the high resistance MTJs overlayed on large negative JMR. The negative JMR and the direction of the switching require the $\mathrm{GdN}$ and $\mathrm{SmN}$ spins to both align with the magnetic field. We suggest that the spin of the SmN aligning with the field is caused by either heavily doped $S m N$, where the additional electrons push the magnetisation from orbital-dominant to spin-dominant, or by spin-transfer torque from orders of magnitude higher current densities 
from hotspots or pinholes in the barrier.

In conclusion, this thesis has studied a wide range of phenomena and properties of the rare earth nitrides and used this knowledge to make magnetic tunnel junctions incorporating $\mathrm{GdN}$ and $\mathrm{SmN}$, and characterised these devices under a large range of current densities, voltages, and magnetic fields.

\subsection{Future outlook}

Further work on $\mathrm{GdN}$ and $\mathrm{SmN}$ based magnetic tunnel junctions could vary the tunnel barrier material. Changing the material in the tunnel barrier could change the surface states formed between the rare earth nitrides and the barrier which can influence the tunnelling mechanism. Less resistive barriers and barriers with defects are more likely to have pinholes or defect mediated tunnelling. A large portion of the more recently published magnetic tunnel junctions use $\mathrm{MgO}$ barriers which display excellent tunnel properties, however making an oxide barrier on the rare earth nitrides is a challenge due to the propensity of the RENs to oxidise in an uncontrolled fashion.

To scale device production the device reproducibility under identical chamber conditions should be investigated. Small differences in chamber conditions, surface purity, and pattern quality will change the resulting device performance. Knowing the growth-to-growth variation in 'identical' device depositions would aid in understanding the differences between devices with intentionally varied properties.

This thesis has focussed mainly on studying the tunnelling in the magnetic tunnel junctions. This is equivalent to studying the readout mode of an MRAM. Further research into the writing of information into the device is needed. The writing to the device could be done for example using an applied field or spin transfer torque [113]. In a magnetic tunnel junction with GdN and SmN electrodes, the magnetic properties of the 
GdN electrode could be tuned to flip magnetisation at a particular field, almost independently of the carrier concentration. The coercive field of the GdN could be tuned to a particular application requiring higher or lower fields to switch. To write to a device using spin torque the current density through the device typically needs to be on the order of $10^{10} \mathrm{~A} / \mathrm{m}^{2}$, often accomplished by making small devices [110].

Moving towards epitaxial devices for increased magnetic ordering necessitates a new manufacturing approach and the development of epitaxially compatible bottom contacts and tunnel barriers. The shadow mask and mounting assembly described in section 3.5 can be manufactured in different materials to allow high-temperature growth. A different approach for patterning epitaxial devices is moving to top-down patterning using etching or milling to remove material after uniform initial film deposition.

In conclusion, the RENs offer a rich system in which to investigate the magnetic and electrical properties of intrinsic ferromagnetic semiconductors. The ability to combine RENs of contrasting magnetic properties bode well not only for the development of magnetic devices but also for investigating and understanding a multitude of interesting magnetic phenomena. The large magnetic response demonstrated in the devices in this thesis together with the dopability of the RENs, and epitaxial compatibility of the RENs with suitable metals and insulators shows promise for making MTJs with excellent switching properties for a variety of future applications. 
Appendices 



\section{Appendix A}

\section{Machine control code}

\section{A.1 Bath cryostat}

\section{A.1.1 Electronics set up instructions}

Power up router. Wait 2 minutes. Connect instruments and computer.

Make sure DHCP is enabled on instrument config.

Check what the ip-addresses of the 3 instruments are, look under instr config on front panel.

$\$$ netstat -nr - grep default

Also check what addresses the arduino and current source are on, they can change when uplugged. \$ ls / dev/tty.*

The sample voltmeter and ammeter are both Tektronix 4040 DMMs.

Can connect via RS232 or ethernet.

The thermometer voltmeter is a FLUKE 8845A multimeter.

Can connect via RS232 or ethernet.

The magnetic field sensor is a Group3 DTM-133 which outputs an analog voltage on the two back pins which is proportional to the applied field. Make sure the sensor is set to FITLER, not HOLD (this only allows you to increase field), TESLA, the correct scale (1.2) and that the comma sign is NOT blinking. 
This is read by an Ardunio UNO (knock-off) which has a simple analog read and send serial data setup, using a switch to send serial data when sent an ' $r$ ' over serial by python. Current source setup:

Keithley 6220 via RS232.

Baudrate 19.2k LF (line feed terminator) No flow control. 8 bits, 1 stop, no parity.

Page 3-15 in manual has programming commands.

Red (high), black (low), green (earth)

Errors:

* timeout: timed out

You forgot to plug in a cable, or the equipment is not on, or the ip-address(es) are incorrect, or the usb address is wrong. The router might not have powered up properly.

* ConnectionRefusedError: [Errno 61] Connection refused

Multimeter is already open, or wasn't closed properly. Try using close_machines() and then re-run the script. If this doesn't work, restart all the multimeters using the front on/off button.

\section{A.1.2 Python code}

This is the python code used to run the bath cryostat electronics on an Ubuntu laptop. The above instructions are listed in the pythonfile.

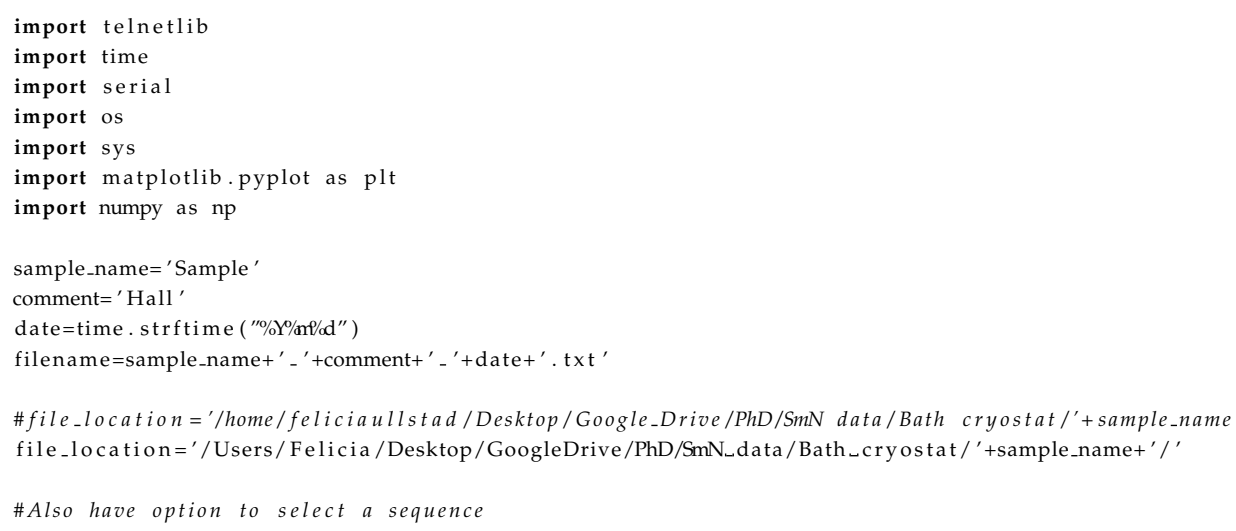




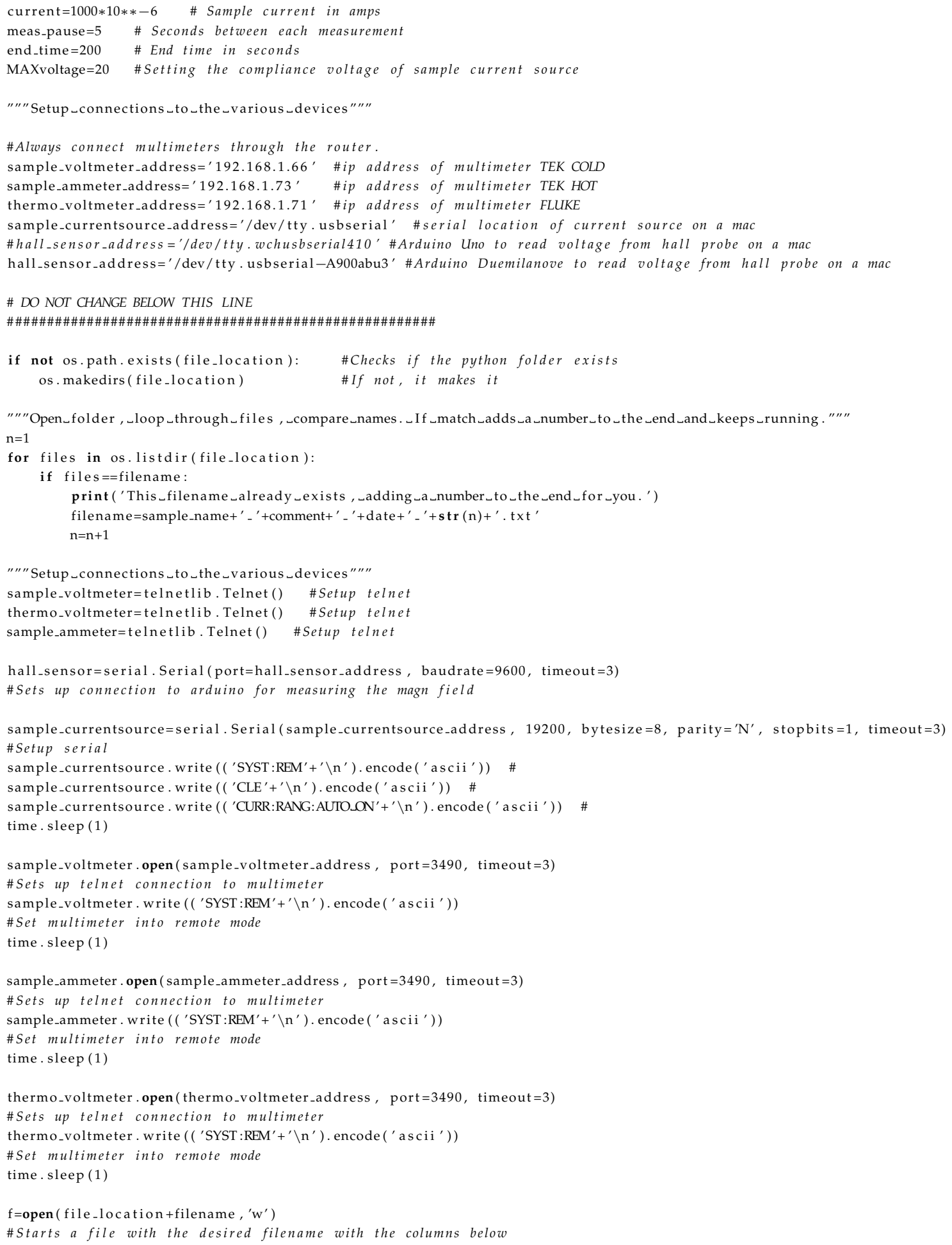




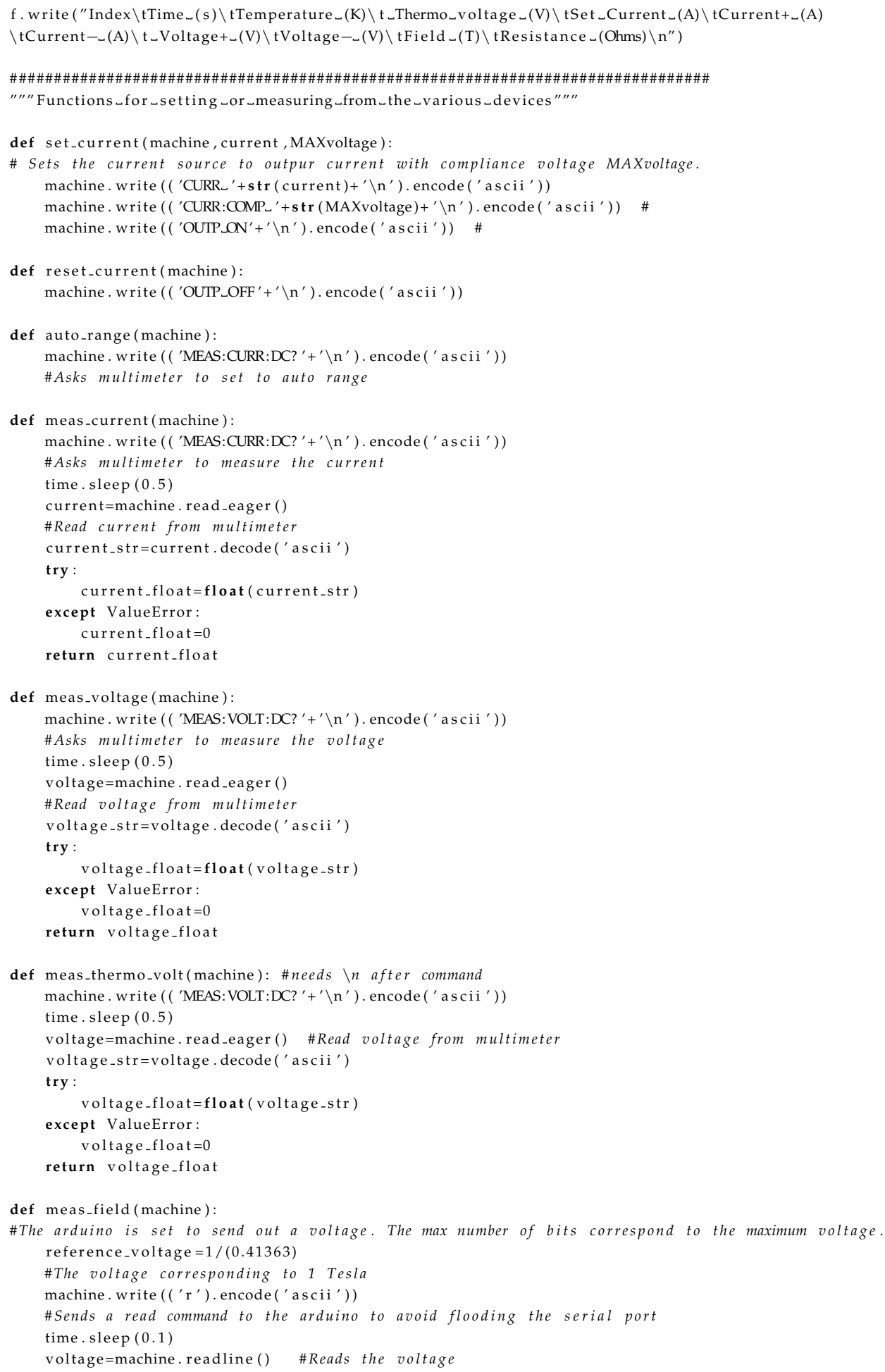




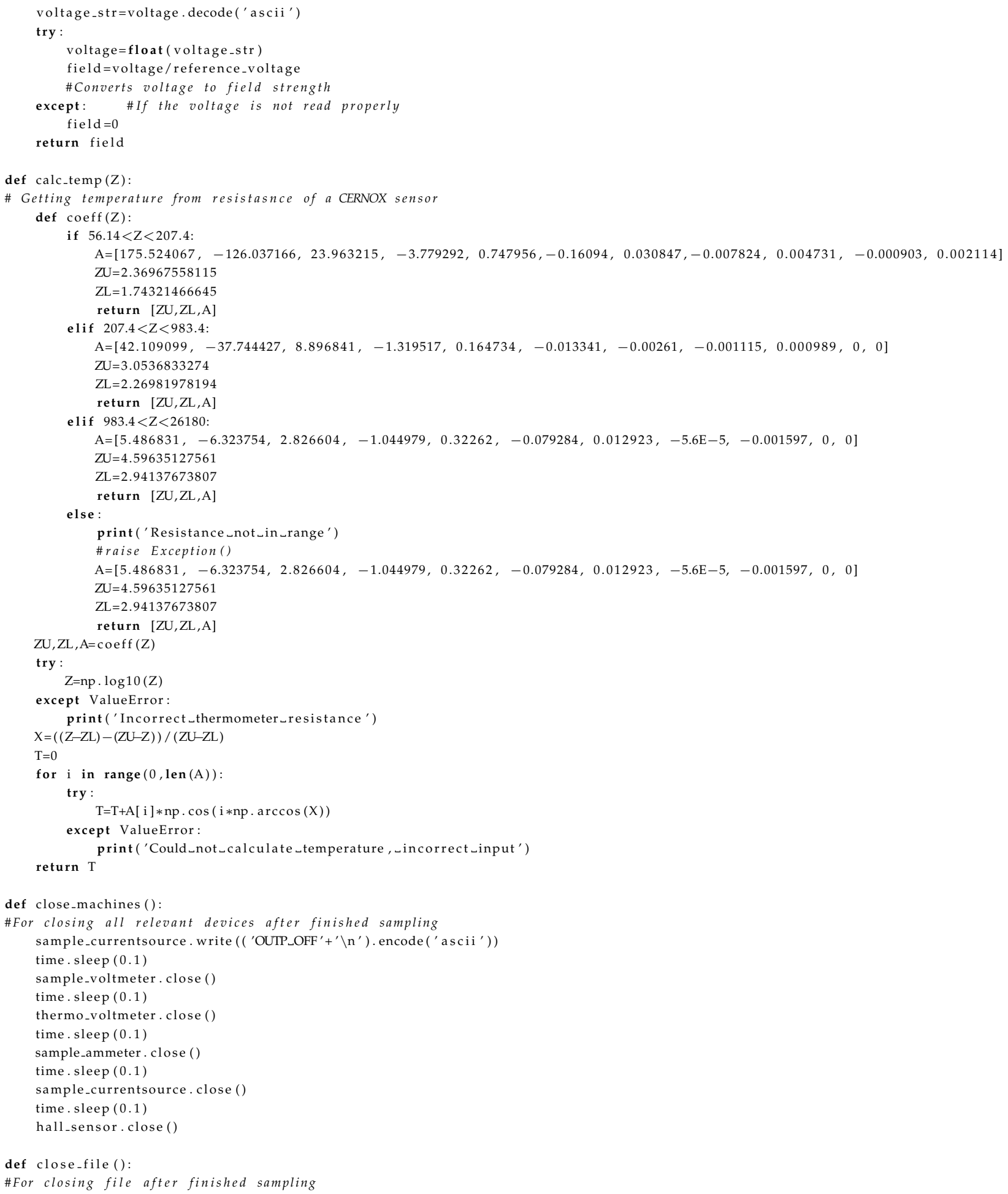


f. close ()

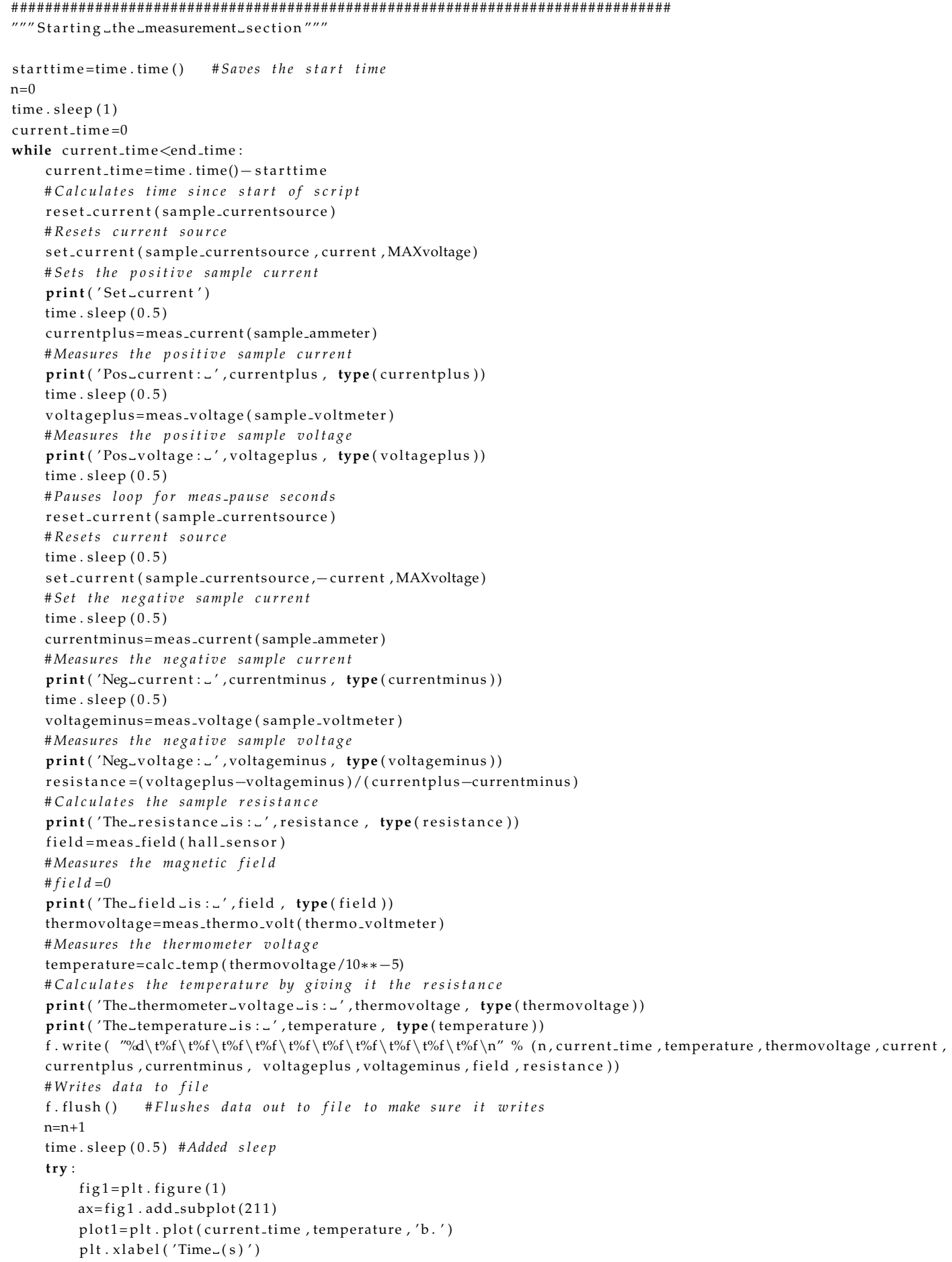




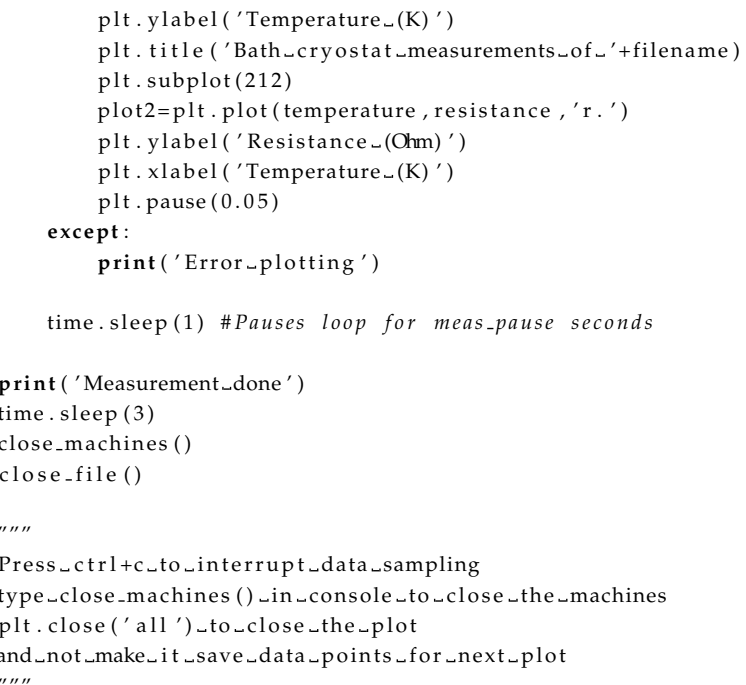

\section{A.1.3 Arduino code}

This is the code running the arduino. The arduino reads the analog output of the hall sensor, converts in to digital, and then sends the value to the computer when requested via serial.

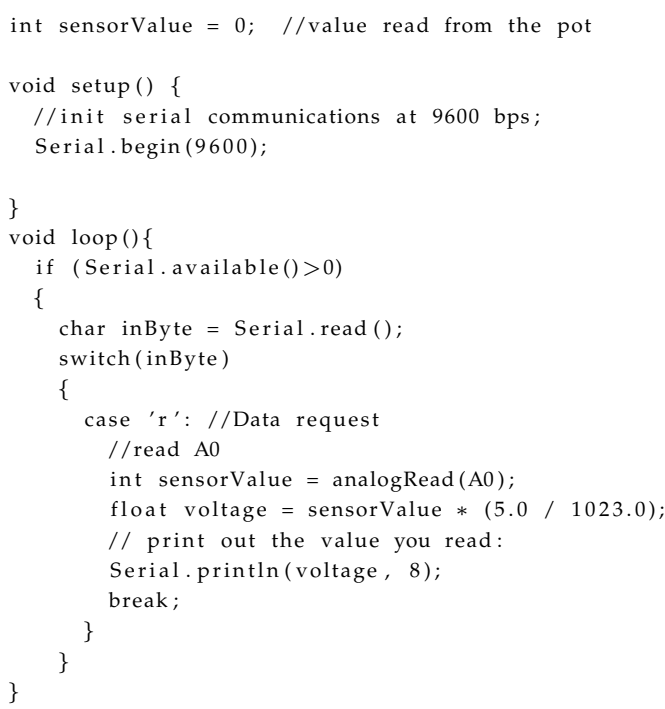




\section{A.2 In-situ electrical measurements Varian}

This python script runs a multimeter and pressure gauge connected to an Ubuntu laptop.

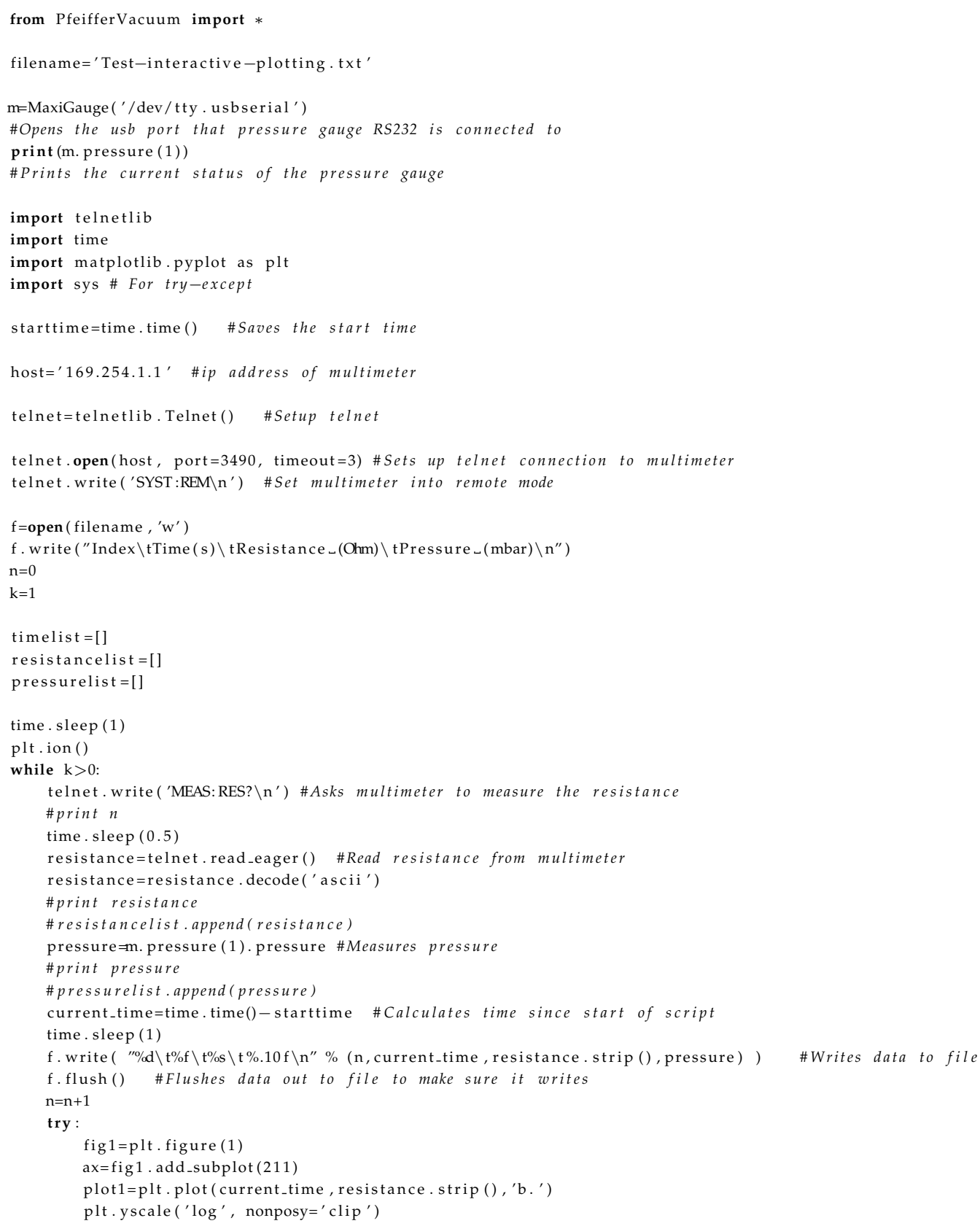


plt. ylim $(10 * *-3,10 * * 10)$ \#Resistance range to be displayed

plt.xlabel ('Time_(s)')

plt . ylabel ('Resistance $($ (Ohm)')

plt. title ('In-situ $\lrcorner$ measurements $\lrcorner$ of ${ }_{\llcorner}$'+filename)

plt. subplot (212)

plot $2=$ plt. plot $($ current_time, pressure , 'r . ')

plt. yscale ('log', nonposy=' clip')

plt. ylabel ('Pressure $\left.\triangle(\text { mbar })^{\prime}\right)$

plt . pause (0.05)

except :

print ('Overload')

telnet.close() \#Closes telnet connection to multimeter

f. close () \#Closes file

" "n"

Press_ctrl+c_to_interrupt_data_sampling

type $\lrcorner$ telnet. close () and

f. close ()

in_console 


\section{Appendix B}

\section{Data analysis code}

\section{B.1 Cryostat auto-batch}

The closed cycle cryostat outputs a textfile for each datapoint in an I-V curve which rapidly means 1000 s of files. This python script takes in which temperatures I-V curves were taken at and which folder the files are in and makes new files with all I-V data points for each temperature. I-V curve data point files are generally 1-3 lines long (depending on how long you make the measurement) so all short files are binned as I-V data while longer files are binned as Restistance vs Temperature files (which are often much longer as the cryostat continously sweeps the temperature).

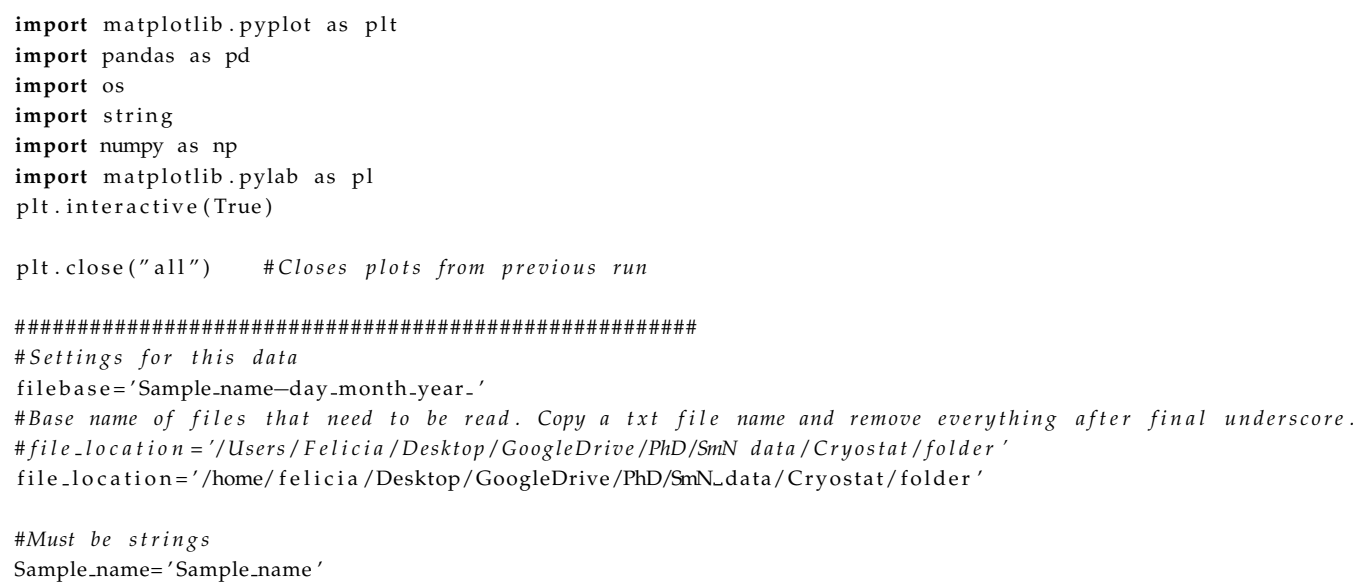


Cryostat_program='RvsT'

IV_temperature_scans $=[4,6,10,15,20,25,30,35,40,50,60,70,100,150,200,300]$

\#Write in the temperatures you used to do IV-scans

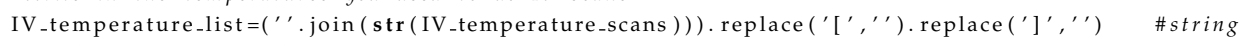

print (IV_temperature_list)

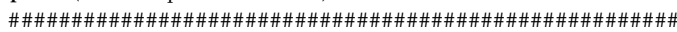

if not os.path.exists(file_location+'-python'): \#Checks if the python folder exists

os . makedirs (file_location+'-python')

\# If not, it makes it

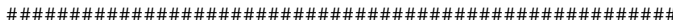

\#Plot settings RvsT

labels $=[$ 'Temperature $\lrcorner \mathrm{A}^{\prime}$, 'Temperature', 'Positive $\lrcorner$ voltage', 'Negative $\lrcorner$ voltage', 'Positive $\lrcorner$ current',

'Negative_current', 'V_limit_reached?', 'Desired_current', 'Resistance', 'Time_(s)']

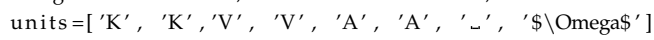

plotx='Temperature' \#Change this to plot other data on the x-axis

ploty='Resistance' \#Change this to plot other data on the y-axis

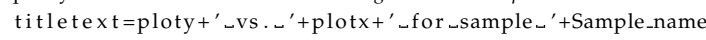

\#Plot settings IV plot

IVplot $x 1={ }^{\prime}$ Positive - voltage ${ }^{\prime}$

IVploty1= 'Positive_current

IVplotx $2=$ 'Negative_voltage

IVploty2 $=^{\prime}$ Negative $\_$current

IVunitx $=$ ' $\mathrm{V}$ '

IVunity $={ }^{\prime} A^{\prime}$

IVtitletext='Voltage_vs ._current_for_sample_' +Sample_name+' _at_-'

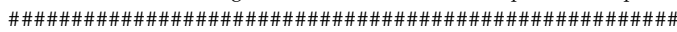

\#Find out which file numbers we want to use for RosT plot

\#and which ones we want for IV plots

\#Find all files in file location

\#Open them all with skiprow 0 to avoid getting wrong column coun

\#if the have one line do nothing

\# if they have 3 lines - check temperature - bin that file number to IV scan at that temperature

\# if they have more than 3 lines - bin that file number to $R$ vs $T$

def stripfile(filename): \#Strips the file name to just the file number

print (filename)

filename $=$ filename . replace $\left({ }^{\prime}, \mathrm{txt}^{\prime}{ }^{\prime},{ }^{\prime \prime}\right)$

if,$\sim$ in filename:

filename $=$ filename . replace $\left({ }^{\prime} \sim{ }^{\prime},{ }^{\prime},{ }^{\prime}\right)$

if ' $\triangle$ ' in filename:

filename=filename , replace $\left(\right.$ ' $\left.^{\prime},{ }^{\prime}, '\right)$

if filebase $!=$ ' '.

filename $=$ filename , replace $\left(\right.$ filebase,${ }^{\prime}$, )

\#print (filename)

filename $=$ int (filename )

return filename

def fullname(filenumber): \#Expands the file number to a full file name

if filenumber $<10$ :

fullname $=$ filebase $+^{\prime}{ }^{\prime}+$ str $($ filenumber $)+{ }^{\prime} . t x t{ }^{\prime}$

else :

fullname $=$ filebase + str $($ filenumber $)+{ }^{\prime} . t_{x t}{ }^{\prime}$

return fullname

\#Create dictinary with keys of file_list_ $290 \mathrm{~K}$ etc and the lists as arguments

def all_file_numbers (file_location, IV_temperature_scans):

\#Finds all the file numbers and checks if they are part of IV scan or RvsT and bins them accordingly

all_filenumbers $=\{\}$

for filename in os.listdir(file_location):

\#print (filename)

if 'details' not in filename: \#To skip the details file that doesn't match the rest

testingfiles=pd.read_csv (file_location+'/'+filename, header=None, delim_whitespace=True, skiprows $=0$ ) 


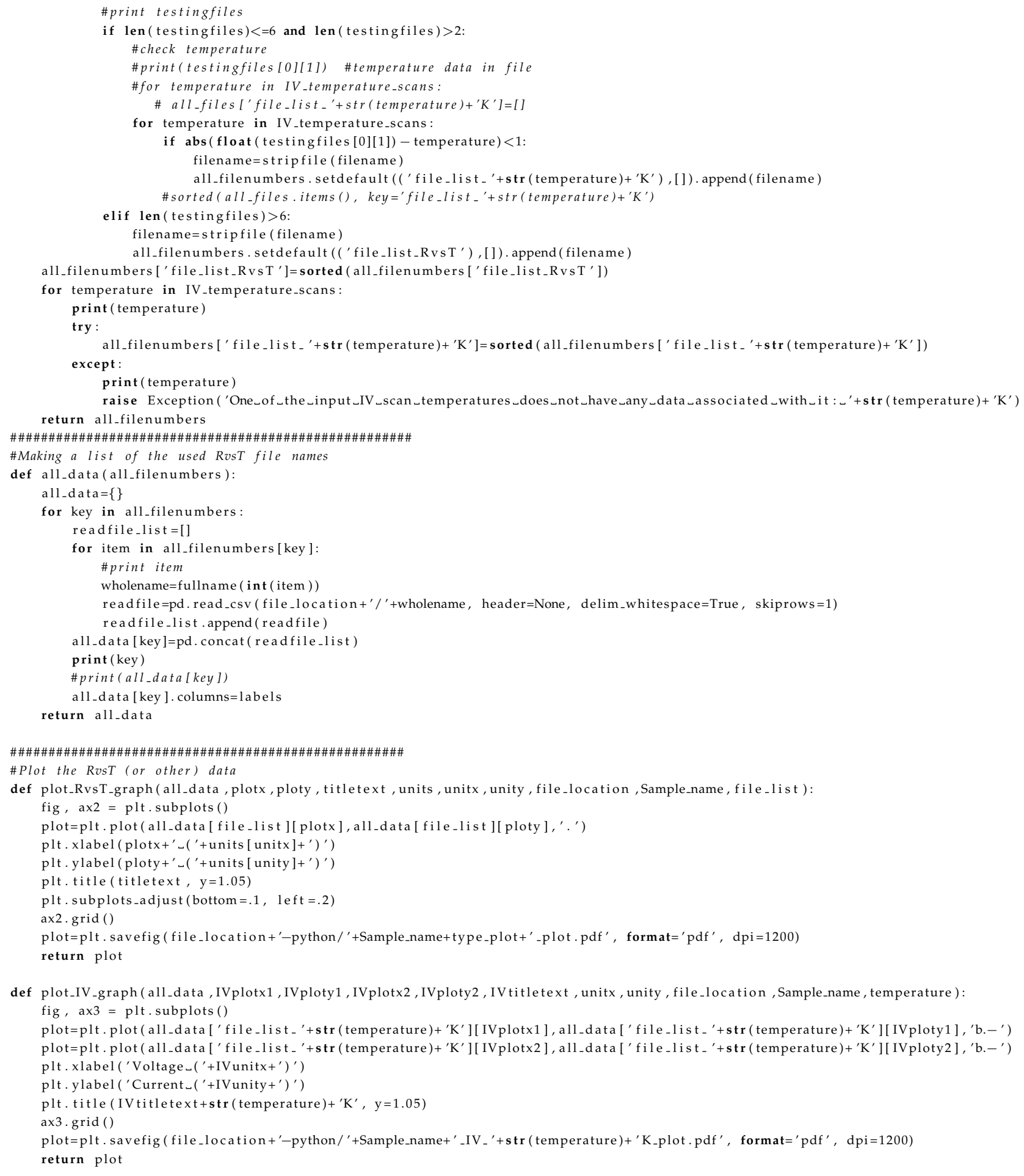




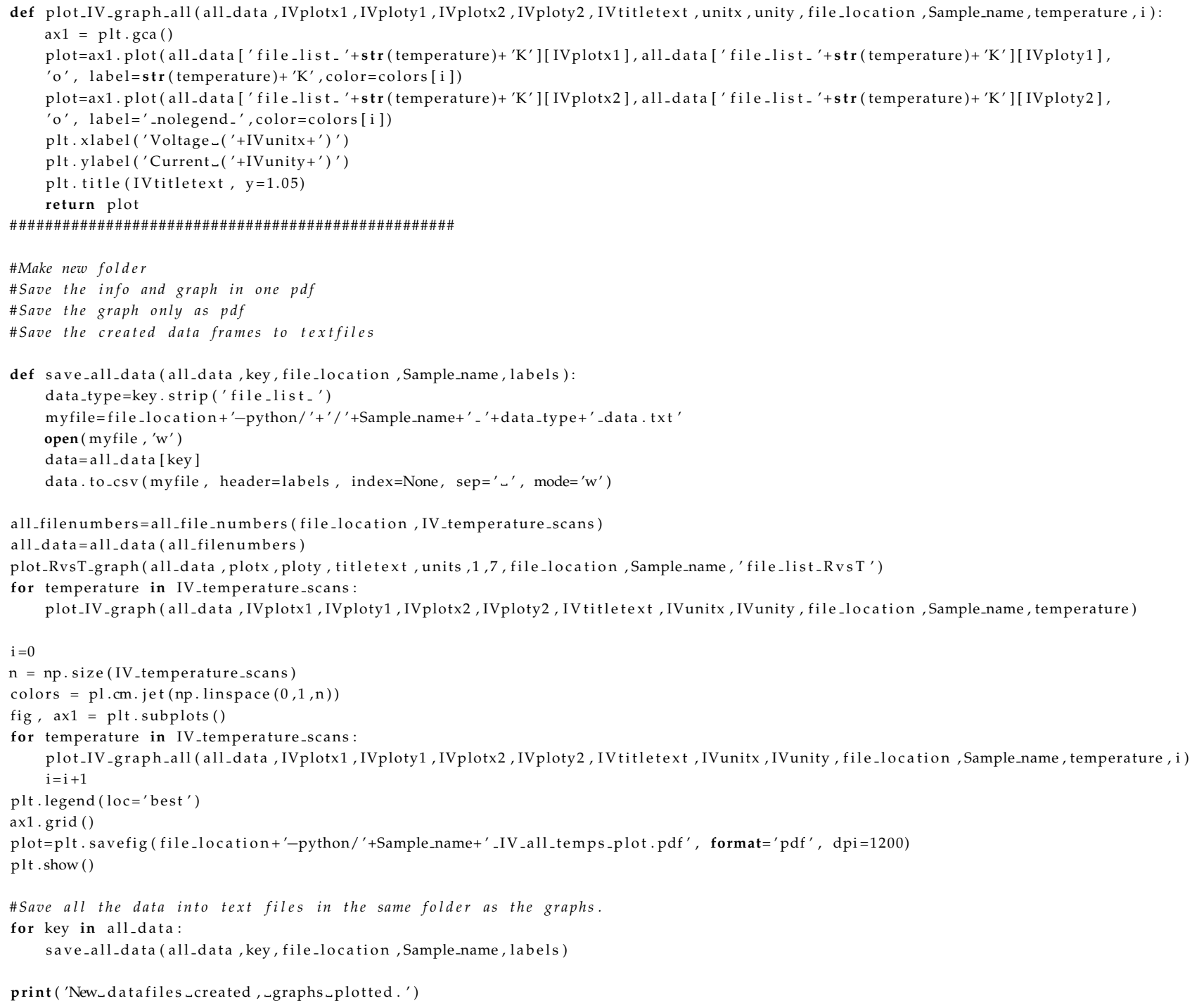

\section{B.2 Simmons model}

Python code for fitting a Simmons model to the J-V data.

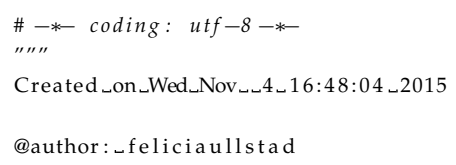




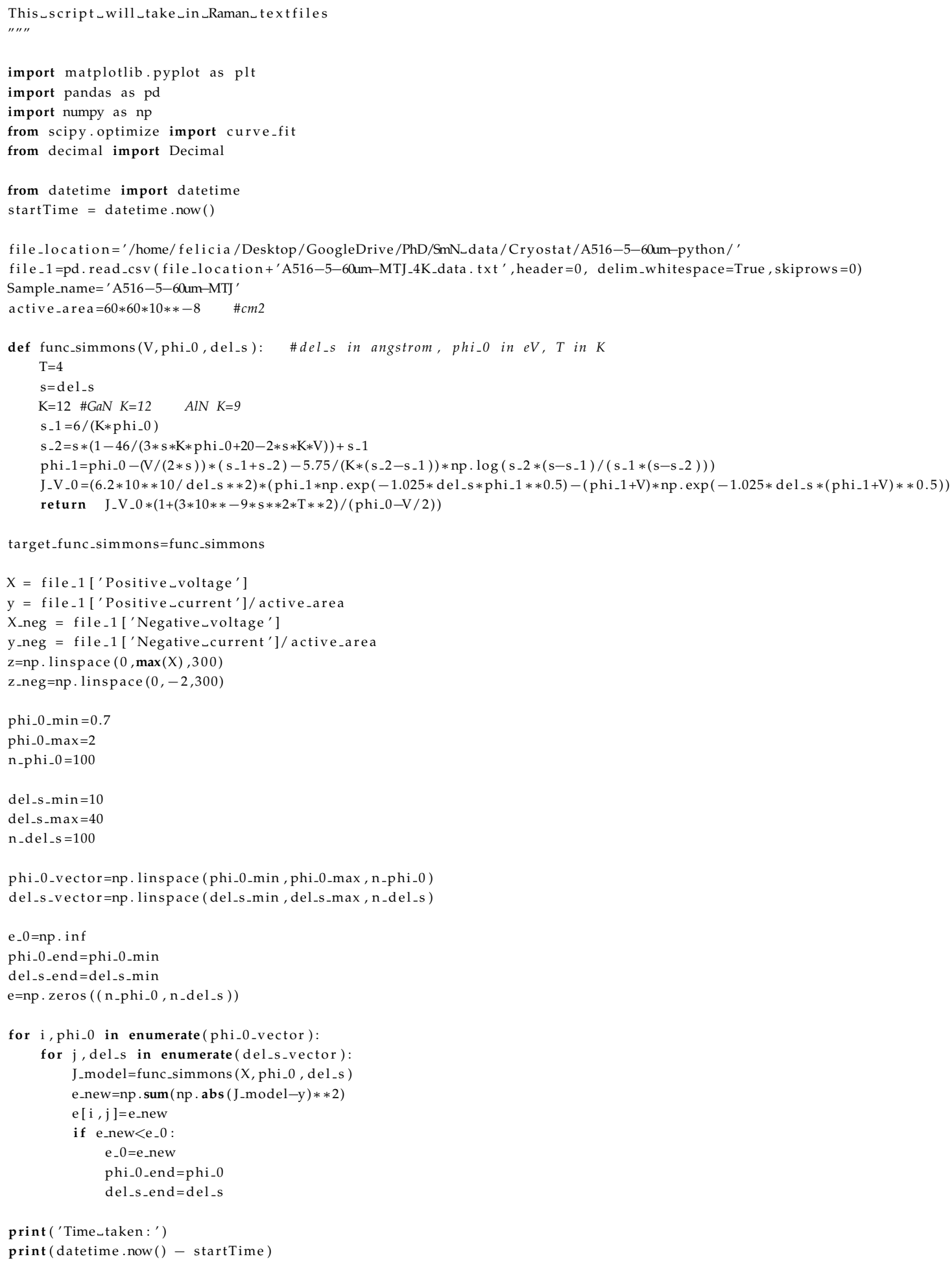


fig, $a x=$ plt.subplots ()

cax $=a x$.imshow $(n p . \log 10($ e $)$, extent $=[$ del_s_min ,del_s_max , phi_0_min ,phi_0_max $]$, aspect="auto" , origin='lower' $)$ fig. colorbar ( cax)

$x y=("\{: .3\} "$. format $($ Decimal (del_s_end $)), "\{: .3\} "$. format $($ Decimal $($ phi_o_end $))$

ax. annotate ('(\%s, $\% s)^{\prime} \% x y, x y=x y$, textcoords='data', fontsize $\left.=14\right)$

limits $=$ ax. axis ()

plt. xlabel ('Barrier_width_(A)', fontsize $=16$ )

plt. ylabel ('Barrier_height_ $(\mathrm{eV})^{\prime}$, , fontsize $\left.=16\right)$

plt. $x$ ticks ( fontsize $=16$, rotation $=0$ )

plt. yticks ( fontsize $=16$, rotation $=0$ )

plt.plot (del_s_end, phi_0_end, '*', markersize $=14$, color $=$ ' white')

ax. axis (limits)

plot=plt.savefig (file_location +'A516-5-60um-IV_4K-low-V-scanning-parameters-plot.pdf',

format='pdf', dpi=1200, bbox_inches='tight', pad_inches $=0.0$ )

plt.style.use('default')

fig, ax1 $=$ plt. subplots ()

ax1.plot $\left(\mathrm{z} * 1000\right.$, target_func_simmons $(\mathrm{z}$, phi_0_end, del_s_end $)$, 'k-_', label='Simmons_fit : - $_{-}+$

" $\{: .3\}$ ". format (Decimal (phi_0_end)) + eV, ' +" $\{: .3\}$ ". format (Decimal (del_s_end )) + 'A')

ax1.plot $\left(X * 1000, y, \quad\right.$ r. ', label='Positive $\left.\_J V^{\prime}\right)$

ax1.plot(-X_neg*1000, -y_neg, 'b.' , label='Negative $\left.\lrcorner J V^{\prime}\right)$

ax1. grid ()

ax1. set_xlabel ('Voltage__ $(\mathrm{mV}))^{\prime}$, fontsize $\left.=16\right)$

ax1. set_ylabel ('Current_density॰ $(\mathrm{A} / \mathrm{cm} 2)$ ', fontsize $=16)$

plt. $x$ ticks ( fontsize $=16$, rotation $=0$ )

plt. yticks ( fontsize $=16$, rotation $=0$ )

plt. legend $\left(\right.$ loc $=$ ' best $\left.^{\prime}\right)$

$\operatorname{ax} 2=\operatorname{ax} 1 . \operatorname{twinx}()$

$\mathrm{mn}, \mathrm{mx}=$ ax1.get_ylim ()

ax2. set_ylim (mn*active_area $* 1000$, mx*active_area $* 1000)$

ax2. set_ylabel ('Current_(mA)', fontsize =16)

plt. yticks (fontsize $=16$, rotation $=0$ )

plot=plt . savefig ( file_location + 'A516-5-60um-IV_4K-fitted-Simmons_plot.pdf',

format='pdf', dpi=1200, bbox_inches='tight', pad_inches $=0.0$ )

fig, ax1 = plt.subplots ()

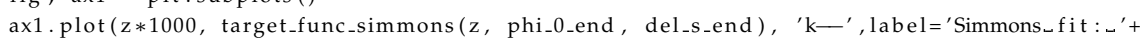

" $\{: .3\}$ ". format (Decimal (phi_0_end )) + 'eV, ' +" $\{: .3\}$ ". format (Decimal (del_s_end )) + 'A')

ax1 $\cdot \operatorname{plot}(X * 1000, y$, 'r.', label='Positive $\left.\lrcorner J V^{\prime}\right)$

ax1.plot $\left(-X \_n e g * 1000,-y \_n e g, \quad ' b .{ }^{\prime}\right.$, label='Negative $\left.\lrcorner J V^{\prime}\right)$

ax1.grid ()

ax1. set_xlabel ('Voltage__ $(\mathrm{mV})$ ', fontsize $=16)$

ax1. set_ylabel ('Current_density॰ $(\mathrm{A} / \mathrm{cm} 2)^{\prime}$, fontsize $\left.=16\right)$

plt. $x$ ticks ( fontsize $=16$, rotation $=0$ )

plt. yticks ( fontsize $=16$, rotation $=0$ )

plt. legend $($ loc = 'best' $)$

plt. yscale ('log')

plt.xscale ('log')

plot=plt . savefig (file_location +'A516-5-60um-IV_4K-fitted-Simmons_plot-loglog ·pdf',

format $=$ 'pdf' $^{\prime}, \mathrm{dpi}=1200$, bbox_inches $={ }^{\prime}$ tight', pad_inches $=0.1$ )

plt. show () 


\section{Appendix C}

\section{Drawings}

This appendix contains mechanical drawings of photo masks, shadow masks, and support pieces for using shadow masks. The designs are all done in Fusion360. The photo mask drawings were redone in layouteditor to be able to be manufactured.

The photomasks were all manufactured by the microfabrication facility at Callaghan Innovation. The shadowmask was manufactured by Veco Precision. The support pieces for the shadowmask were manufactured by mechanical workshop at the School of Chemical and Physical Sciences at Victoria University of Wellington. 


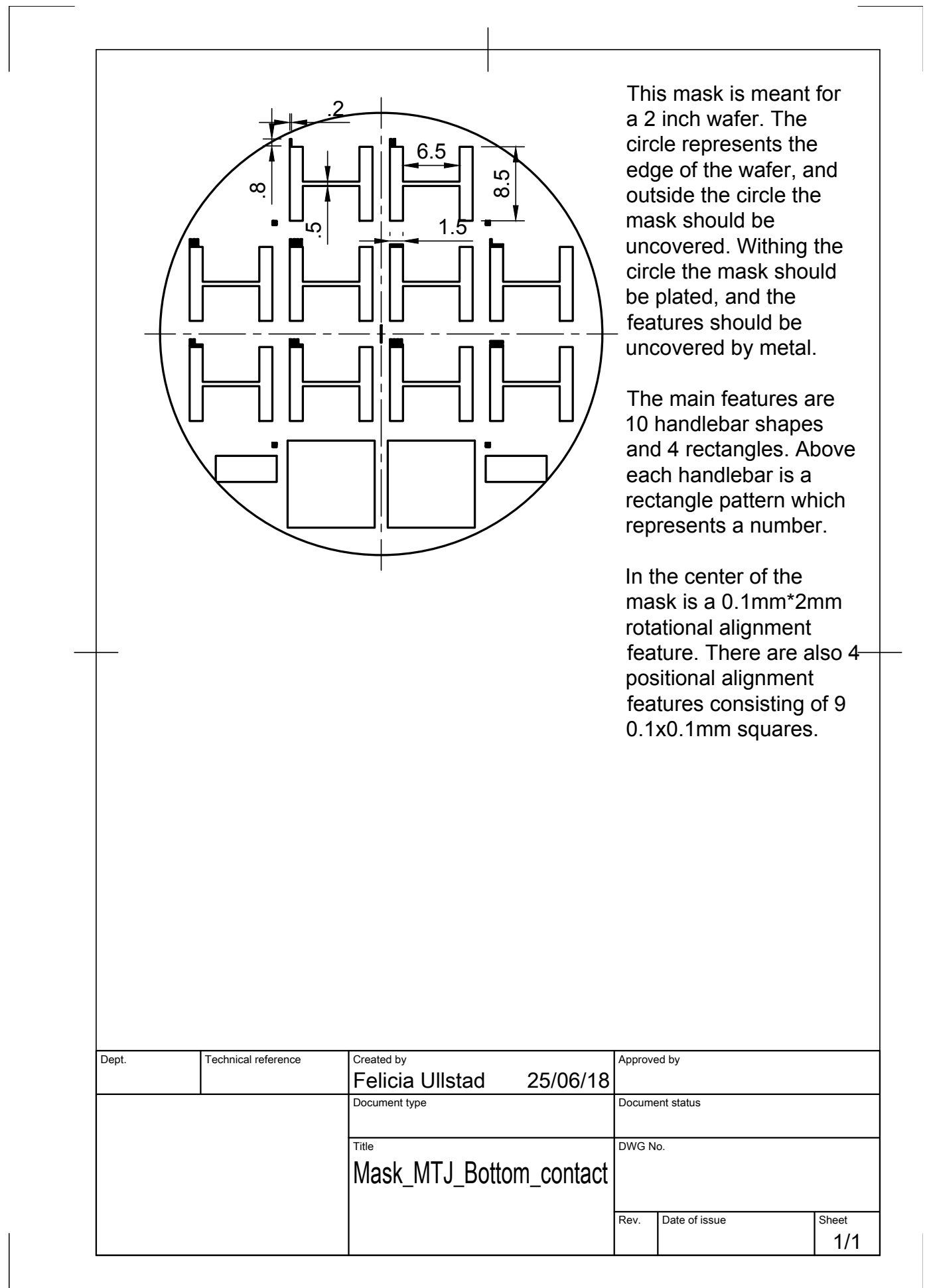

Figure C.1: Photomask for bottom contacts layer. Designed by me using Fusion360 and layouteditor. Manufactured by Callaghan Innovation. 


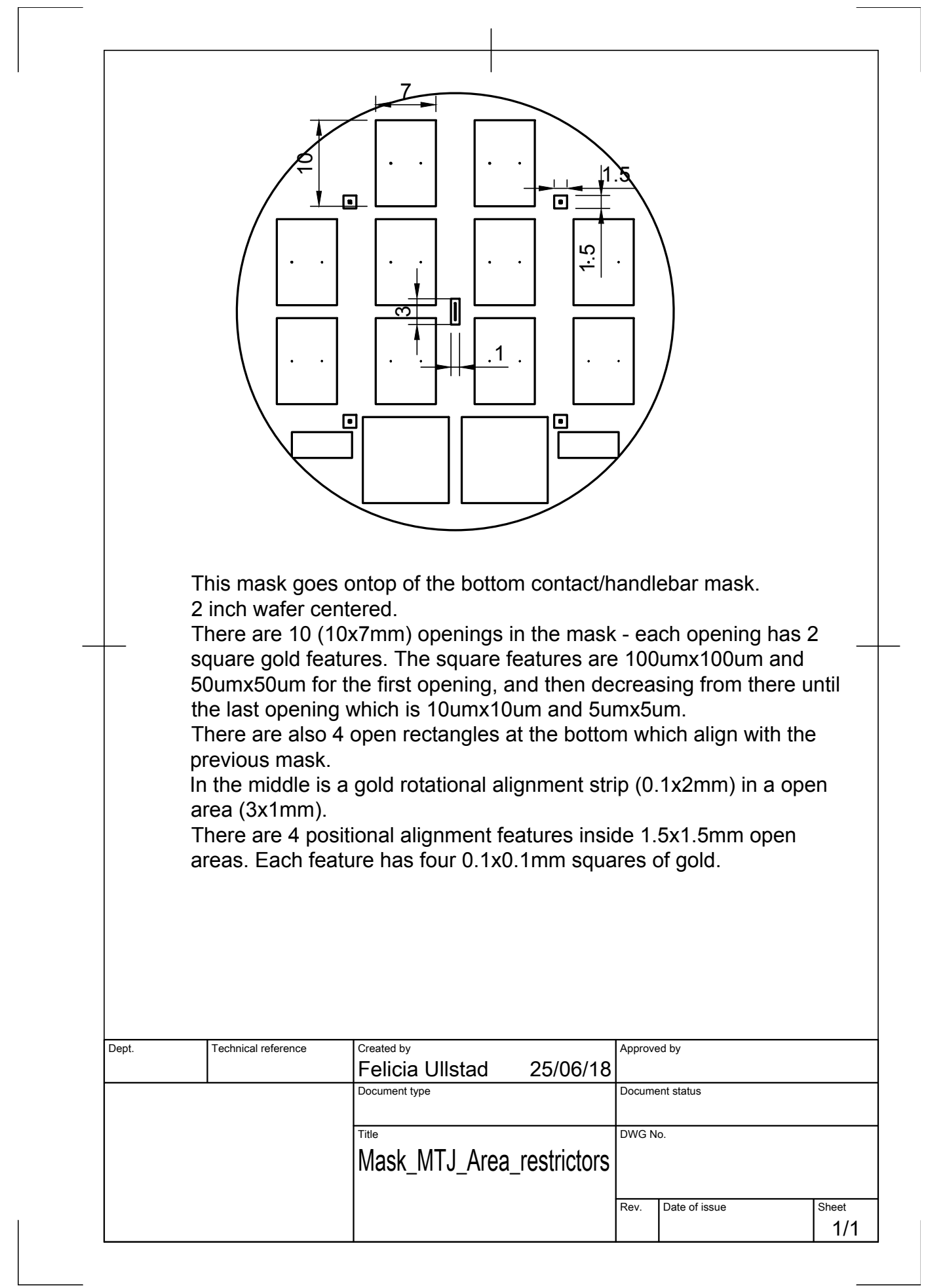

Figure C.2: Photomask for area restriction layer. Designed by me using Fusion360 and layouteditor. Manufactured by Callaghan Innovation. 


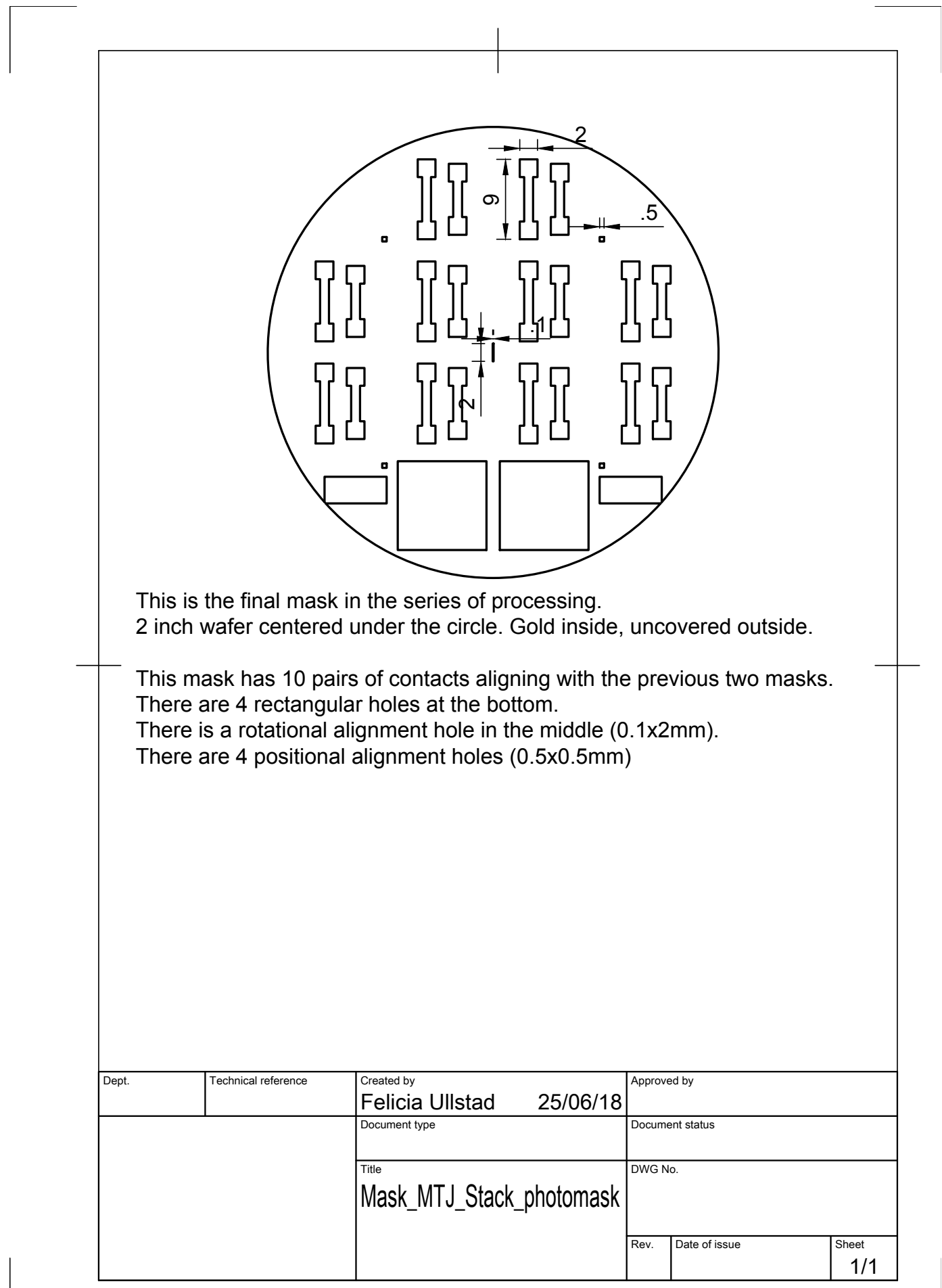

Figure C.3: Photomask for MTJ stack layer. Designed by me using Fusion360 and layouteditor. Manufactured by Callaghan Innovation. 


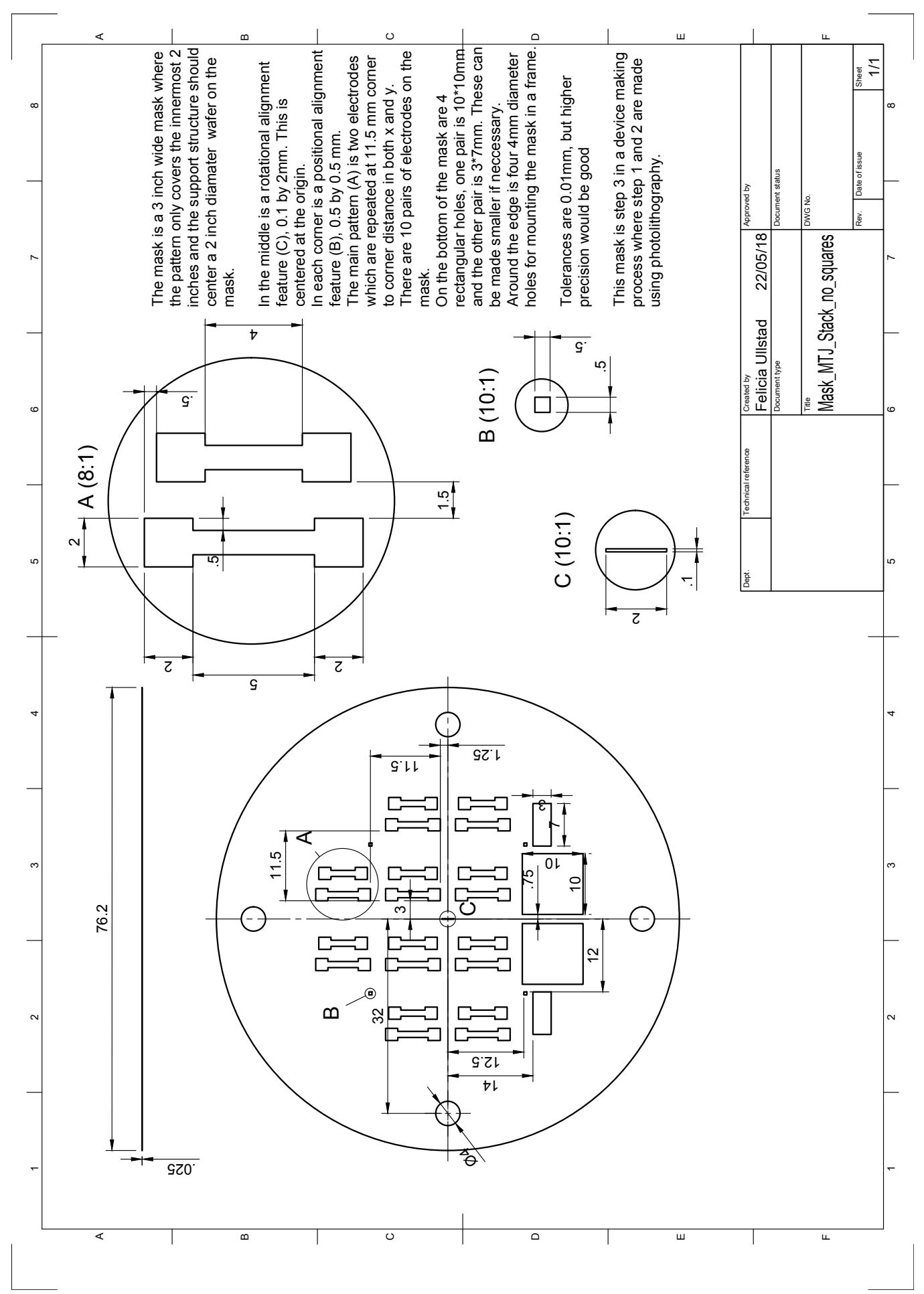

Figure C.4: $20 \mu \mathrm{m}$ thick shadowmask. Designed by me using Fusion360. Manufactured by Veco Precision. 


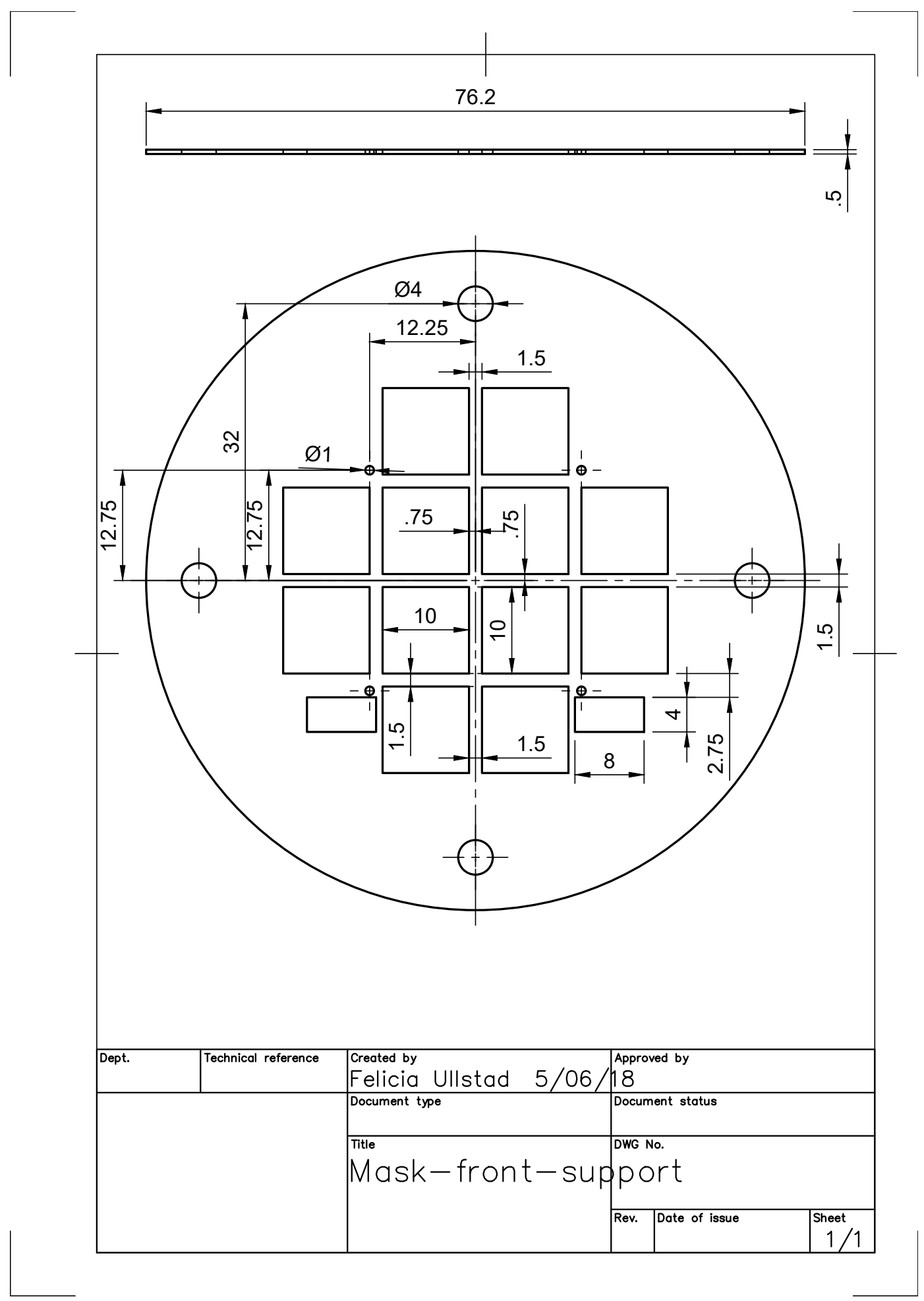

Figure C.5: Support structure for a 2 inch wafer held behind the shadowmask foil. Designed by me using Fusion360. Manufactured by Veco Precision. 


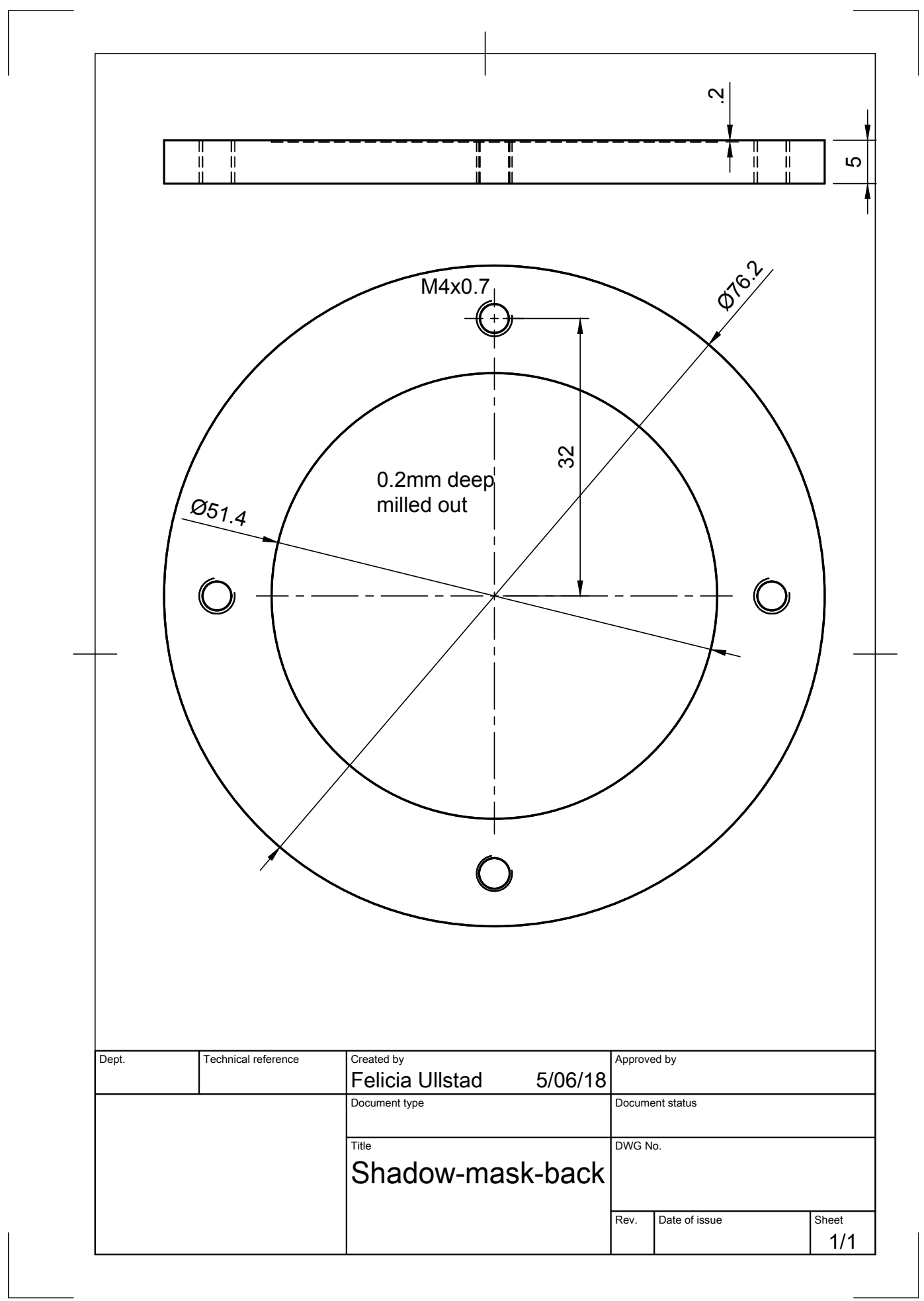

Figure C.6: Front support structure for the shadow mask and a 2 inch wafer. Designed by me using Fusion360. Manufactured by Veco Precision. 


\section{Bibliography}

[1] F. Natali, B. J. Ruck, N. O. V. Plank, H. J. Trodahl, S. Granville, C. Meyer, and W. R. L. Lambrecht, "Rare-earth mononitrides," Progress in Materials Science, vol. 58, no. 8, pp. 1316-1360, 2013. 1, $8,10,11,19,47,69,83,90,92,97,112,162$

[2] S. Granville, B. J. Ruck, F. Budde, A. Koo, D. J. Pringle, F. Kuchler, A. R. H. Preston, D. H. Housden, N. Lund, A. Bittar, G. V. M. Williams, and H. J. Trodahl, "Semiconducting ground state of GdN thin films," Physical Review B, vol. 73, p. 235335, 2006. 1, 16, 19, 20, $21,22,47,53,75,76,77$

[3] C. Meyer, B. J. Ruck, A. R. H. Preston, S. Granville, G. V. M. Williams, and H. J. Trodahl, "Magnetic properties of ErN films," Journal of Magnetism and Magnetic Materials, vol. 322, no. 14, pp. 1973-1978, 2010. 1

[4] D. Le Binh, B. J. Ruck, F. Natali, H. Warring, H. J. Trodahl, E.-M. Anton, C. Meyer, L. Ranno, F. Wilhelm, and A. Rogalev, "Europium nitride: A novel diluted magnetic semiconductor," Physical Review Letters, vol. 111, p. 167206, 2013. 1

[5] A. R. H. Preston, S. Granville, D. H. Housden, B. Ludbrook, B. J. Ruck, H. J. Trodahl, A. Bittar, G. V. M. Williams, J. E. Downes, A. DeMasi, Y. Zhang, K. E. Smith, and W. R. L. Lambrecht, “Comparison 
between experiment and calculated band structures for $\mathrm{DyN}$ and SmN," Physical Review B, vol. 76, p. 245120, 2007. 1

[6] K. Senapati, M. G. Blamire, and Z. H. Barber, "Spin-filter Josephson junctions.," Nature Materials, vol. 10, no. 11, pp. 849-52, 2011. 2

[7] A. Pal, K. Senapati, Z. H. Barber, and M. G. Blamire, "Electric-fielddependent spin polarization in GdN spin filter tunnel junctions," Advanced Materials, vol. 25, pp. 5581-5585, 2013. 2, 101, 157

[8] P. K. Muduli, A. Pal, and M. G. Blamire, "Crossover from diffusive to tunneling regime in $\mathrm{NbN}$ / DyN / NbN ferromagnetic semiconductor tunnel junctions," Physical Review B, vol. 89, no. 094414, pp. 1-5, 2014. 2

[9] H. Warring, H. J. Trodahl, N. O. V. Plank, F. Natali, S. Granville, and B. J. Ruck, "Magnetic Tunnel Junctions Incorporating a NearZero-Moment Ferromagnetic Semiconductor," Physical Review Applied, vol. 6, p. 044002, 2016. 2, 101, 103, 135, 154, 160

[10] Z. Zhang and M. G. Lagally, "Atomistic Processes in the Early Stages of Thin-Film Growth," Science, vol. 276, pp. 377-383, 1997. 7, 53

[11] M. H. Grabow and G. H. Gilmer, "Thin film growth modes, wetting and cluster nucleation," Surface Science, vol. 194, pp. 333-346, 1988. 7,8

[12] U. W. Pohl, Epitaxy of Semiconductors. Berlin: Springer-Verlag Berlin Heidelberg, 1 ed., 2013. 7,8

[13] S. C. Seel and C. V. Thompson, "Tensile stress generation during island coalescence for variable island-substrate contact angle," Journal of Applied Physics, vol. 93, no. 11, pp. 9038-9042, 2003. 8 
[14] I. Petrov, P. B. Barna, L. Hultman, and J. E. Greene, "Microstructural evolution during film growth," Journal of Vacuum Science E Technology $A$, vol. 21, no. 5, 2003. 8

[15] M. Ohring, The Materials Science of Thin Films. London: Academic Press, 2 ed., 2002. 8

[16] P. Larson, W. R. L. Lambrecht, A. Chantis, and M. Van Schilfgaarde, "Electronic structure of rare-earth nitrides using the LSDA+U approach: Importance of allowing $4 \mathrm{f}$ orbitals to break the cubic crystal symmetry," Physical Review B, vol. 75, pp. 1-14, 2007. 8, 16

[17] F. Hulliger, Rare earth pnictides, vol. 4. Amsterdam: North-Holland Publishing Company, 1 ed., 1979. 8, 10

[18] R. Marchand, Ternary and higher order nitride materials, vol. 25. Elsevier, 1998. 10

[19] R. Didchenro and F. P. Gortsbma, "Some Electric and Magnetic Properties of Rare Earth Monosulfides and Nitrides," Journal of Physics and Chemistry of Solids, vol. 24, pp. 863-870, 1963. 10

[20] H. Imamura, T. Imahashi, M. Zaimi, and Y. Sakata, "Preparation and characteristics of various rare earth nitrides," Journal of Alloys and Compounds, vol. 451, pp. 636-639, 2008. 10

[21] B. M. Ludbrook, I. L. Farrell, M. Kuebel, B. J. Ruck, a. R. H. Preston, H. J. Trodahl, L. Ranno, R. J. Reeves, and S. M. Durbin, "Growth and properties of epitaxial GdN," Journal of Applied Physics, vol. 106, p. $063910,2009.10,12,16,24,114$

[22] Z. Fang, P. A. Williams, R. Odedra, H. Jeon, and R. J. Potter, "Gadolinium nitride films deposited using a PEALD based process," Journal of Crystal Growth, vol. 338, pp. 111-117, 2012. 10 
[23] F. Natali, B. Ludbrook, J. Galipaud, N. Plank, S. Granville, A. Preston, B. L. Do, J. Richter, I. Farrell, R. Reeves, S. Durbin, J. Trodahl, and B. Ruck, "Epitaxial growth and properties of GdN, EuN and SmN thin films," Physica Status Solidi (C), vol. 9, no. 3-4, pp. 605$608,2012.11,12$

[24] C. Meyer, B. J. Ruck, J. Zhong, S. Granville, A. R. H. Preston, G. V. M. Williams, and H. J. Trodahl, "Near-zero-moment ferromagnetism in the semiconductor SmN," Physical Review B, vol. 78, pp. 1-5, 2008. $12,13,16,17,79,112$

[25] W. R. McKenzie, P. R. Munroe, F. Budde, B. J. Ruck, S. Granville, and H. J. Trodahl, "TEM characterisation of GdN thin films," Current Applied Physics, vol. 6, pp. 407-410, 2006. 12

[26] J. W. Gerlach, J. Mennig, and B. Rauschenbach, “Epitaxial gadolinium nitride thin films," Applied Physics Letters, vol. 90, no. 061919, 2007. 12

[27] C. N. Christodoulou and T. Takeshita, "Reaction of samarium with hydrogen and nitrogen samarium oxides," Journal of Alloys and Compounds, vol. 190, pp. 99-106, 1992. 12

[28] H. A. Eick, N. C. Baenziger, and L. Eyring, “The Preparation, Crystal Structure and Some Properties of $\mathrm{SmN}$, EuN and $\mathrm{YbN}$," Journal of the American Chemical Society, vol. 78, no. 23, pp. 5987-5989, 1956. 12

[29] F. Natali, B. J. Ruck, H. J. Trodahl, D. L. Binh, S. Vezian, B. Damilano, Y. Cordier, F. Semond, and C. Meyer, "Role of magnetic polarons in ferromagnetic GdN," Physical Review B, vol. 87, no. 035202, 2013. 13, $14,15,20,22,88,94,112,114$

[30] F. Natali, N. O. V. Plank, J. Galipaud, B. J. Ruck, H. J. Trodahl, F. Semond, S. Sorieul, and L. Hirsch, "Epitaxial growth of GdN on sili- 
con substrate using an AlN buffer layer," Journal of Crystal Growth, vol. 312, pp. 3583-3587, 2010. 15, 16, 78, 114

[31] K. Senapati, T. Fix, M. E. Vickers, M. G. Blamire, and Z. H. Barber, "Magnetic exchange hardening in polycrystalline GdN thin films.," Journal of Physics: Condensed Matter, vol. 22, p. 302003, 2010. 16

[32] B. J. Ruck, F. Natali, N. O. V. Plank, B. Do Le, M. Azeem, M. Alfheid, C. Meyer, and H. J. Trodahl, "The influence of nitrogen vacancies on the magnetic behaviour of rare-earth nitrides," Physica B, vol. 407, pp. 2954-2956, 2012. 16

[33] F. Natali, S. Vézian, S. Granville, B. Damilano, H. J. Trodahl, E. M. Anton, H. Warring, F. Semond, Y. Cordier, S. V. Chong, and B. J. Ruck, "Molecular beam epitaxy of ferromagnetic epitaxial GdN thin films," Journal of Crystal Growth, vol. 404, pp. 146-151, 2014. 16

[34] K. Khazen, H. J. von Bardeleben, J. L. Cantin, A. Bittar, S. Granville, H. J. Trodahl, and B. J. Ruck, "Ferromagnetic resonance study of GdN thin films with bulk and extended lattice constants," Physical Review B, vol. 74, p. 245330, 2006. 16, 88, 94, 148

[35] N. N. Som, V. H. Mankad, S. D. Dabhi, A. Patel, and P. K. Jha, "Magnetic behavior study of samarium nitride using density functional theory," Journal of Magnetism and Magnetic Materials, vol. 448, pp. 186-191, 2018. 16

[36] E.-M. Anton, B. J. Ruck, C. Meyer, F. Natali, H. Warring, F. Wilhelm, A. Rogalev, V. N. Antonov, and H. J. Trodahl, "Spin/orbit moment imbalance in the near-zero moment ferromagnetic semiconductor SmN," Physical Review B, vol. 87, p. 134414, 2013. 16, 20

[37] J. F. McNulty, E.-M. Anton, B. J. Ruck, F. Natali, H. Warring, F. Wilhelm, A. Rogalev, M. Medeiros Soares, N. B. Brookes, and H. J. Trodahl, "Twisted phase of the orbital-dominant ferromagnet SmN in 
a GdN/SmN heterostructure," Physical Review B, vol. 91, p. 174426, 2015. 16, 17, 18, 154

[38] J. F. McNulty, Twisted magnetization phases in orbital-dominant rareearth nitrides. Doctor of philosophy, Victoria University of Wellington, 2015. 16, 18

[39] E.-M. Anton, S. Granville, A. Engel, S. V. Chong, M. Governale, U. Zülicke, A. G. Moghaddam, H. J. Trodahl, F. Natali, S. Vézian, and B. J. Ruck, "Superconductivity in the ferromagnetic semiconductor samarium nitride," Physical Review B, vol. 94, p. 024106, 2016. $20,21,53,91$

[40] F. Leuenberger, A. Parge, W. Felsch, K. Fauth, and M. Hessler, "GdN thin films: Bulk and local electronic and magnetic properties," Physical Review B, vol. 72, p. 014427, 2005. 22, 23, 24

[41] S. Granville, C. Meyer, A. R. H. Preston, B. M. Ludbrook, B. J. Ruck, H. J. Trodahl, T. R. Paudel, and W. R. L. Lambrecht, “Vibrational properties of rare-earth nitrides: Raman spectra and theory," Physical Review B, vol. 79, p. 054301, 2009. 47

[42] M. Katsura, "Thermodynamics of nitride and hydride formation by the reaction of metals with flowing $\mathrm{NH}_{3}$," Journal of Alloys and Compounds, vol. 182, no. 1, pp. 91-102, 1992. 47

[43] M. Itoh, K.-i. Machida, M. Yamamoto, K. Hirose, and G.-y. Adachi, "Reversible nitrogen absorption and desorption on rare earth-iron intermetallic compounds," Journal of Alloys and Compounds, vol. 364, pp. 208-213, 2004. 47, 51

[44] H. Jehn and P. Ettmayer, "The molybdenum-nitrogen phase diagram," Journal of The Less-Common Metals, vol. 58, pp. 85-98, 1978. 47,68 
[45] T. L. Cottrell, The Strengths of Chemical Bonds. London: Butterworths, 2nd ed., 1958. 48

[46] D. A. Gonnella, The Fundamental Science of Nitrogen-Doping of Niobium Superconducting Cavities. Doctor of philosophy, Cornell University, 2016. 48

[47] E. Metin and O. T. Inal, "Formation and growth of iron nitrides during ion-nitriding," Journal of Materials Science, vol. 22, pp. 2783-2788, 1987. 48

[48] R. A. Pasternak and B. Evans, "Adsorption, absorption, and degassing in the Oxygen-Niobium System at Very Low Pressure," Journal of Electrochemical Society, vol. 114, no. 5, pp. 452-457, 1967. 48

[49] D. Pye, An Introduction to Nitriding. Materials Park, Ohio: American Technical Publishers Ltd, 1 ed., 2003. 48

[50] W. Lengauer, "Properties of bulk $\delta$ - $\mathrm{TiN}_{1-x}$ prepared by nitrogen diffusion into titanium metal," Journal of Alloys and Compounds, vol. 186, pp. 293-307, 1992. $48,61,71$

[51] M. Yang, Nitriding - fundamentals, modeling and process optimization. $\mathrm{PhD}$ thesis, Worcester Polytechnic Institute, 2012. 48

[52] Z. Cao, Thin film growth: physics, materials science and applications. Woodhead Publishing Limited, 1 ed., 2011. 48

[53] R. Wei, J. J. Vajo, J. N. Matossian, P. J. Wilbur, J. A. Davis, D. L. Williamson, and G. A. Collins, "A comparative study of beam ion implantation, plasma ion implantation and nitriding of AISI 304 stainless steel," Surface and Coatings Technology, vol. 83, pp. 235-242, 1996. 49,62 
[54] M. Cao, R. Wang, X. Fang, Z. Cui, T. Chang, and H. Yang, “Preparing $\gamma-\mathrm{Fe}_{4} \mathrm{~N}$ ultrafine powder by twice-nitriding method," Powder Technology, vol. 115, no. 1, pp. 96-98, 2001. 49

[55] Y. Ogino, T. Yamasaki, N. Atzumi, and K. Yoshioka, "Nitriding of Transition Metal Powders by Ball Milling in Nitrogen Gas," 1993. 49,63

[56] B. J. Jaques, D. D. Osterberg, G. A. Alanko, S. Tamrakar, C. R. Smith, M. F. Hurley, and D. P. Butt, "Kinetics of the nitridation of dysprosium during mechanochemical processing," Journal of Alloys and Compounds, vol. 619, pp. 253-261, 2015. 49, 63

[57] A. I. Gusev, “Nitrogen Partial Pressure of Stoichiometric and Nonstoichiometric Titanium, Vanadium and Niobium Nitrides and Carbonitrides," Physica Status Solidi (B), vol. 209, p. 267, 1998. 49

[58] J. A. Venables, G. D. T. Spiller, and M. Hanbucken, “Nucleation and growth of thin films," Reports on Progress in Physics, vol. 47, pp. 399$459,1984.53$

[59] K. D. Ralston, N. Birbilis, and C. H. J. Davies, "Revealing the relationship between grain size and corrosion rate of metals," Scripta Materialia, vol. 63, pp. 1201-1204, 2010. 53, 72

[60] B. B. Dixit, P. D. Vyas, W. S. Khokle, K. Mahadevan, and H. N. Acharya, "Electrical and structural characteristics of oxides grown from polycrystalline silicon," Bulletin of Materials Science, vol. 8, no. 3, pp. 307-313, 1986. 53, 72

[61] T. Telbizova, T. Chevolleau, and W. Möller, "Nitrogen incorporation and loss during ion nitriding of $\mathrm{Al}$, " Nuclear Instruments and Methods in Physics Research B, vol. 184, pp. 347-353, 2001. 60 
[62] B. E. Deal and A. S. Grove, "General relationship for the thermal oxidation of silicon," Journal of Applied Physics, vol. 36, no. 12, pp. 37703778, 1965. 61

[63] S. Lambie, $N_{2}$ Dissociation Over Selected Pure Rare Earth Surfaces. Masters, University of Otago, 2018. 62

[64] M. Azeem, B. J. Ruck, B. Do Le, H. Warring, H. J. Trodahl, N. M. Strickland, A. Koo, V. Goian, and S. Kamba, "Optical response of DyN," Journal of Applied Physics, vol. 113, p. 203509, 2013. 63

[65] V. Koeninger, H. Uchida, and H. Uchida, "Nitrogen absorption and desorption of $\mathrm{Sm}_{2} \mathrm{Fe}_{17}$ in ammonia and hydrogen atmospheres," Journal of Alloys and Compounds, vol. 222, no. 1-2, pp. 117-122, 1995. 68

[66] A. H. Daane, R. E. Rundle, H. G. Smith, and F. H. Spedding, "The crystal structure of samarium," Acta Crystallographica, vol. 7, pp. 532-535, 1954. 69

[67] S. Giddey, S. P. S. Badwal, and A. Kulkarni, "Review of electrochemical ammonia production technologies and materials," International Journal of Hydrogen Energy, vol. 38, no. 34, pp. 14576-14594, 2013. 72

[68] S. Enzo, G. Fagherazzi, A. Benedetti, and S. Polizzi, "A profilefitting procedure for analysis of broadened $\mathrm{X}$-ray diffraction peaks. I. Methodology," Journal of Applied Crystallography, vol. 21, 1988. 84

[69] K. Senapati, T. Fix, M. E. Vickers, M. G. Blamire, and Z. H. Barber, "Structural evolution and competing magnetic orders in polycrystalline GdN films," Physical Review B, vol. 83, no. 1, p. 014403, 2011. $85,89,94$ 
[70] F. Ullstad, J. R. Chan, H. Warring, N. Plank, B. Ruck, J. Trodahl, and F. Natali, "Ohmic contacts of Au and Ag metals to n-type GdN thin films," AIMS Materials Science, vol. 2, no. 2, pp. 79-85, 2015. 91

[71] W. F. Holmes-Hewett, F. H. Ullstad, B. J. Ruck, F. Natali, and H. J. Trodahl, "Anomalous Hall effect in $\mathrm{SmN}$ : Influence of orbital magnetism and 4f-band conduction," Physical Review B, vol. 98, p. 235201, 2018. 91

[72] N. O. V. Plank, F. Natali, J. Galipaud, J. H. Richter, M. Simpson, H. J. Trodahl, and B. J. Ruck, "Enhanced Curie temperature in N-deficient GdN," Applied Physics Letters, vol. 98, p. 112503, 2011. 92

[73] J. Miller, Tunnelling in rare-earth nitride structures. Masters, Victoria University of Wellington, 2018. 92, 93, 96, 101, 135, 159

[74] E. Y. Tsymbal, O. N. Mryasov, and P. R. LeClair, "Spin-dependent tunnelling in magnetic tunnel junctions," Journal of Physics: Condensed Matter, vol. 15, no. 4, 2003. 97, 99

[75] M. Julliere, “Tunneling between ferromagnetic films," Physics Letters, vol. 54A, no. 3, pp. 225-226, 1975. 99

[76] T. Miyazaki and N. Tezuka, "Giant magnetic tunneling effect in $\mathrm{Fe} / \mathrm{Al}_{2} \mathrm{O}_{3} / \mathrm{Fe}$ junction," Journal of Magnetism and Magnetic Materials, vol. 139, pp. L231-L234, 1995. 100

[77] S. S. P. Parkin, C. Kaiser, A. Panchula, P. M. Rice, B. Hughes, M. Samant, and S.-H. Yang, "Giant tunnelling magnetoresistance at room temperature with $\mathrm{MgO}$ (100) tunnel barriers," Nature Materials, vol. 3, pp. 862-867, 2004. 100

[78] S. Ikeda, J. Hayakawa, Y. Ashizawa, Y. M. Lee, K. Miura, H. Hasegawa, M. Tsunoda, F. Matsukura, and H. Ohno, "Tunnel magnetoresistance of $604 \%$ at $300 \mathrm{~K}$ by suppression of Ta diffusion 
in $\mathrm{CoFeB} / \mathrm{MgO} / \mathrm{CoFeB}$ pseudo-spin-valves annealed at high temperature," Applied Physics Letters, vol. 93, p. 082508, 2008. 100

[79] N. García, "Conducting ballistic magnetoresistance and tunneling magnetoresistance: Pinholes and tunnel barriers," Applied Physics Letters, vol. 77, no. 9, pp. 1351-1353, 2000. 100, 126

[80] D. A. Rabson, B. J. Jönsson-Åkerman, A. H. Romero, R. Escudero, C. Leighton, S. Kim, and I. K. Schuller, "Pinholes may mimic tunneling," Journal of Applied Physics, vol. 89, pp. 2786-2790, 2001. 100, 126

[81] Z. S. Zhang and D. A. Rabson, "Electrical and thermal modeling of the non-Ohmic differential conductance in a tunnel junction containing a pinhole," Journal of Applied Physics, vol. 95, no. 2, pp. 557-560, 2004. 100, 126, 159

[82] J. J. Åkerman, I. V. Roshchin, J. M. Slaughter, R. W. Dave, and I. K. Schuller, "Origin of temperature dependence in tunneling magnetoresistance," Europhysics Letters, vol. 63, no. 1, pp. 104-110, 2003. 100

[83] J. M. Teixeira, J. Ventura, J. P. Araujo, J. B. Sousa, P. Wisniowski, S. Cardoso, and P. P. Freitas, "Resonant tunneling through electronic trapping states in thin $\mathrm{MgO}$ magnetic junctions," Physical Review Letters, vol. 106, no. 19, pp. 1-4, 2011. 100, 135, 158

[84] Y. Ando, J. Murai, H. Kubota, and T. Miyazaki, "Magnon-assisted inelastic excitation spectra of a ferromagnetic tunnel junction," Journal of Applied Physics, vol. 87, no. 9, pp. 5209-5211, 2000. 100

[85] B. B. Nelson-Cheeseman, F. J. Wong, R. V. Chopdekar, E. Arenholz, and Y. Suzuki, "Room temperature magnetic barrier layers in magnetic tunnel junctions," Physical Review B, vol. 81, no. 21, pp. 1-7, 2010. 100 
[86] B. B. Nelson-Cheeseman, R. V. Chopdekar, L. M. B. Alldredge, J. S. Bettinger, E. Arenholz, and Y. Suzuki, "Probing the role of the barrier layer in magnetic tunnel junction transport," Physical Review B, vol. 76 , no. 22 , pp. $2-5,2007.100,157,158$

[87] V. Garcia, H. Jaffrès, M. Eddrief, M. Marangolo, V. H. Etgens, and J. M. George, "Resonant tunneling magnetoresistance in MnAs/IIIV/MnAs junctions," Physical Review B, vol. 72, no. 8, pp. 2-5, 2005. 100

[88] C. De Buttet, M. Hehn, F. Montaigne, C. Tiusan, G. Malinowski, A. Schuhl, E. Snoeck, and S. Zoll, "Low-resistance magnetic tunnel junctions with an $\mathrm{MgO}-\mathrm{Al}_{2} \mathrm{O}_{3}$ composite tunnel barrier: Asymmetric transport characteristics and free electron modeling of a selflimited oxidation bilayer," Physical Review B, vol. 73, no. 10, pp. 1-7, 2006. $100,101,158$

[89] J. J. Åkerman, J. M. Slaughter, R. W. Dave, and I. K. Schuller, “Tunneling criteria for magnetic-insulator-magnetic structures," Applied Physics Letters, vol. 79, no. 19, pp. 3104-3106, 2001. 100

[90] J. Schmalhorst, A. Thomas, S. Kämmerer, O. Schebaum, D. Ebke, M. D. Sacher, G. Reiss, A. Hütten, A. Turchanin, A. Gölzhäuser, and E. Arenholz, "Transport properties of magnetic tunnel junctions with $\mathrm{Co}_{2} \mathrm{MnSi}$ electrodes: The influence of temperature-dependent interface magnetization and electronic band structure," Physical Review B, vol. 75, no. 1, pp. 1-10, 2007. 100, 156

[91] J. Zhang and R. M. White, "Voltage dependence of magnetoresistance in spin dependent tunneling junctions," Journal of Applied Physics, vol. 83, no. 11, pp. 6512-6514, 1998. 100, 158 
[92] R. Guerrero, F. G. Aliev, Y. Tserkovnyak, T. S. Santos, and J. S. Moodera, "Shot noise in magnetic tunnel junctions: Evidence for sequential tunneling," Physical Review Letters, vol. 97, no. 26, 2006. 100, 158

[93] J. S. Moodera, J. Nowak, and R. J. M. V. D. Veerdonk, "Interface Magnetism and Spin Wave Scattering in Ferromagnet-InsulatorFerromagnet Tunnel Junctions," Physical Review Letters, vol. 80, no. 13, pp. 2941-2944, 1998. 100, 156, 159

[94] G. G. Cabrera and N. García, "Low voltage I-V characteristics in magnetic tunneling junctions," Applied Physics Letters, vol. 80, no. 10, pp. $1782-1784,2002.100$

[95] S.-J. Ahn, T. Kato, H. Kubota, Y. Ando, and T. Miyazaki, "Biasvoltage dependence of magnetoresistance in magnetic tunnel junctions grown on $\mathrm{Al}_{2} \mathrm{O}_{3}$ (0001) substrates," Applied Physics Letters, vol. 86 , no. $102506,2005.100$

[96] A. Kalitsov, P.-J. Zermatten, F. Bonell, G. Gaudin, S. Andrieu, C. Tiusan, M. Chshiev, and J. P. Velev, "Bias dependence of tunneling magnetoresistance in magnetic tunnel junctions with asymmetric barriers," Journal of Physics Condensed Matter, vol. 25, no. 49, 2013. 100, 156,158

[97] A. Pal and M. G. Blamire, "Large interfacial exchange fields in a thick superconducting film coupled to a spin-filter tunnel barrier," Physical Review B, vol. 92, no. 18, p. 180510 (R), 2015. 101

[98] A. Kandala, A. Richardella, D. W. Rench, D. M. Zhang, T. C. Flanagan, and N. Samarth, "Growth and characterization of hybrid insulating ferromagnet-topological insulator heterostructure devices," Applied Physics Letters, vol. 103, no. 20, p. 202409, 2013. 101 
[99] H. Warring, DEVICE APPLICATIONS OF RARE-EARTH NITRIDES. Doctor of philosophy, Victoria University of Wellington, 2016. 101, $102,135,154,160$

[100] G. Scheunert, C. Ward, W. R. Hendren, A. A. Lapicki, R. Hardeman, M. Mooney, M. Gubbins, and R. M. Bowman, "Influence of strain and polycrystalline ordering on magnetic properties of high moment rare earth metals and alloys," Journal of Physics D: Applied Physics, vol. 47, p. 415005, 2014. 112

[101] S. Cwik, S. M. J. Beer, S. Hoffmann, M. Krasnopolski, D. Rogalla, H.W. Becker, D. Peeters, A. Ney, and A. Devi, "Integrating AlN with GdN Thin Films in an in Situ CVD Process: Influence on the Oxidation and Crystallinity of GdN," ACS Applied Materials $\mathcal{E}$ Interfaces, vol. 9, pp. 27036-27044, 2017. 114

[102] R. J. Gambino, T. R. McGuire, H. A. Alperin, and S. J. Pickart, “Magnetic properties and structure of $\mathrm{GdN}$ and $\mathrm{GdN}_{1-x} \mathrm{O}_{x}$," Journal of Applied Physics, vol. 41, pp. 933-934, 1970. 114, 115

[103] J. Y. Park, M. J. Baek, E. S. Choi, S. Woo, J. H. Kim, T. J. Kim, J. C. Jung, S. Chae, Y. Chang, and G. H. Lee, "Paramagnetic Ultrasmall Gadolinium Oxide Nanoparticles as Advanced T1 MRI Contrast Agent: Account for Large Diameter, Longitudinal Relaxivity, Optimal Particle and In Vivo T1 MR Images," ACS Nano, vol. 3, no. 11, pp. 3663-3669, 2009. 114, 115

[104] S. Hazarika, N. Paul, and D. Mohanta, "Rapid hydrothermal route to synthesize cubic-phase gadolinium oxide nanorods," Bulletin of Materials Science, vol. 37, no. 4, pp. 789-796, 2014. 115

[105] H. Yoshitomi, S. Kitayama, T. Kita, O. Wada, M. Fujisawa, H. Ohta, and T. Sakurai, "Optical and magnetic properties in epitaxial GdN thin films," Physical Review B, vol. 83, no. 15, p. 155202, 2011. 118 
[106] H. J. Trodahl, A. R. H. Preston, J. Zhong, B. J. Ruck, N. M. Strickland, C. Mitra, and W. R. L. Lambrecht, "Ferromagnetic redshift of the optical gap in GdN," Physical Review B, vol. 76, p. 085211, 2007. 118

[107] C. A. Dartora and G. G. Cabrera, "Spin-dependent transmission coefficients for magnetic tunnel junctions: Transport properties and temperature dependence," Physical Review B, vol. 72, p. 014424, 2005. 118

[108] J. G. Simmons, "Generalized Formula for the Electric Tunnel Effect between Similar Electrodes Separated by a Thin Insulating Film," Journal of Applied Physics, vol. 34, no. 6, pp. 1793-1803, 1963. 130

[109] I. B. Rowena, S. L. Selvaraj, and T. Egawa, "Buffer thickness contribution to suppress vertical leakage current with high breakdown field $(2.3 \mathrm{MV} / \mathrm{cm})$ for $\mathrm{GaN}$ on $\mathrm{Si}$," IEEE Electron Device Letters, vol. 32, no. 11, pp. 1534-1536, 2011. 150

[110] C.-Y. You, "Critical current density for spin transfer torque switching with composite free layer structure," Current Applied Physics, vol. 10, pp. 952-956, 2009. 156, 167

[111] Y. S. Choi, K. Tsunekawa, Y. Nagamine, and D. Djayaprawira, "Transmission electron microscopy study on the polycrystalline $\mathrm{CoFeB} / \mathrm{MgO} / \mathrm{CoFeB}$ based magnetic tunnel junction showing a high tunneling magnetoresistance, predicted in single crystal magnetic tunnel junction," Journal of Applied Physics, vol. 101, no. 1, p. $013907,2007.159$

[112] B. Liu, Y. Hua, Z. Dong, P. K. Tan, Y. Zhao, Z. Mo, J. Lam, and Z. H. Mai, "The overview of the impacts of electron radiation on semiconductor failure analysis by sem, fib and tem," 2018 IEEE International Symposium on the Physical and Failure Analysis of Integrated Circuits (IPFA), pp. 1-6, 2018. 162 
[113] J. G. J. Zhu and C. Park, "Magnetic tunnel junctions," Materials Today, vol. 9, no. 11, pp. 36-45, 2006. 166 